\section{Pacific Northwest}

National Laboratory

Operated by Battelle for the

U.S. Department of Energy

\title{
Entry Boreholes Summary Report for the Waste Treatment Plant \\ Seismic Boreholes Project
}

Re-Issue

\author{
J. A. Horner
}

February 2007

Prepared by Gram, Inc. and Fluor Hanford, Inc.

for the Pacific Northwest National Laboratory

under Contract DE-AC05-76RL01830

with the U.S. Department of Energy 


\title{
DISCLAIMER
}

This report was prepared as an account of work sponsored by an agency of the United States Government. Neither the United States Government nor any agency thereof, nor Battelle Memorial Institute, nor any of their employees, makes any warranty, express or implied, or assumes any legal liability or responsibility for the accuracy, completeness, or usefulness of any information, apparatus, product, or process disclosed, or represents that its use would not infringe privately owned rights. Reference herein to any specific commercial product, process, or service by trade name, trademark, manufacturer, or otherwise does not necessarily constitute or imply its endorsement, recommendation, or favoring by the United States Government or any agency thereof, or Battelle Memorial Institute. The views and opinions of authors expressed herein do not necessarily state or reflect those of the United States Government or any agency thereof.

\author{
PACIFIC NORTHWEST NATIONAL LABORATORY \\ operated by \\ BATTELLE \\ for the \\ UNITED STATES DEPARTMENT OF ENERGY \\ under Contract DE-AC05-76RL01830
}

Printed in the United States of America
Available to DOE and DOE contractors from the
Office of Scientific and Technical Information,
P.O. Box 62, Oak Ridge, TN 37831-0062;
ph: (865) 576-8401
fax: (865) 576-5728
email: reports@adonis.osti.gov

\author{
Available to the public from the National Technical Information Service, \\ U.S. Department of Commerce, 5285 Port Royal Rd., Springfield, VA 22161 \\ ph: (800) 553-6847 \\ fax: (703) 605-6900 \\ email: orders@ntis.fedworld.gov \\ online ordering: http://www.ntis.gov/ordering.htm
}

This document was printed on recycled paper. 
WMP-32119

Revision 0

RE-ISSUE

\section{Entry Boreholes Summary Report for the Waste Treatment Plant Seismic Boreholes Project}

Prepared for the U.S. Department of Energy

Assistant Secretary for Environmental Management

Project Hanford Management Contractor for the

U.S. Department of Energy under Contract DE-AC06-96RL13200

FLUOR

P.O. Box 1000

Richland, Washington

Approved for Public Release: Further Dissemination Unlimited 
WMP-32119

Revision 0

RE-ISSUE

\section{Entry Boreholes Summary Report for the Waste Treatment Plant Seismic Boreholes Project}

Document Type: TR

Program/Project: WMP

J. Horner

Gram, inc.

Date Published

February 2007

Prepared for the U.S. Department of Energy

Assistant Secretary for Environmental Management

Project Hanford Management Contractor for the

U.S. Department of Energy under Contract DE-AC06-96RL13200

\section{FLUOR.}

P.O. Box 1000

Richland, Washington

R. A- Lasdal $\frac{0 z / 07 / 2007}{\text { Date }}$ 
WMP-32119

Revision 0

TRADEMARK DISCLAIMER

Reference herein to any specific commercial product, process,

or service by trade name, trademark, manufacturer, or

otherwise, does not necessarily constitute or imply its

endorsement, recommendation, or favoring by the United

States Government or any agency thereof or its contractors or

subcontractors.

This report has been reproduced from the best available copy.

Printed in the United States of America 
WMP-32119, Rev. 0

\section{APPROVAL PAGE}

Title: $\quad$ Entry Boreholes Summary Report for the Waste Treatment Plant Seismic Boreholes Project

\section{Approvals:}

L. C. Swanson

Manager, Geosciences, Groundwater Remediation Project
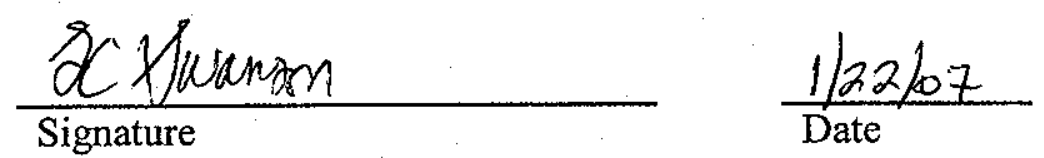

J. A. Winterhalder

Environmental Compliance Officer, Groundwater Remediation Project

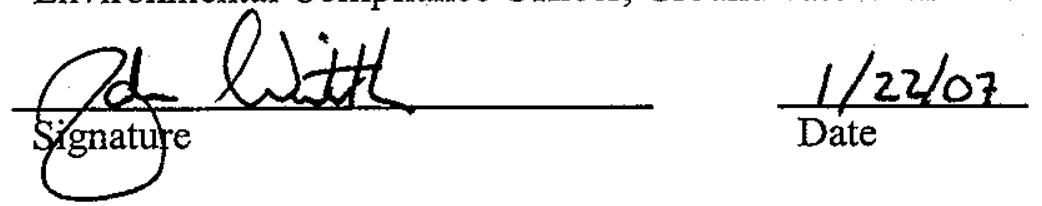

C. S. Wright

Task Lead, Groundwater Remediation Project

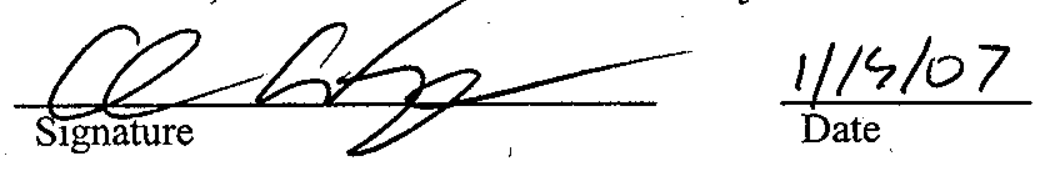


WMP-32119, Rev. 0

This page intentionally left blank. 


\section{CONTENTS}

1.0 INTRODUCTION

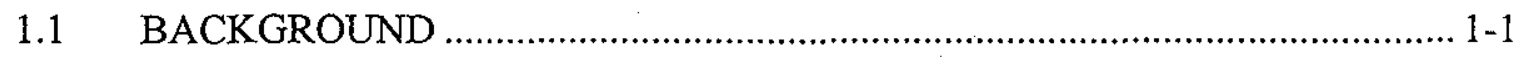

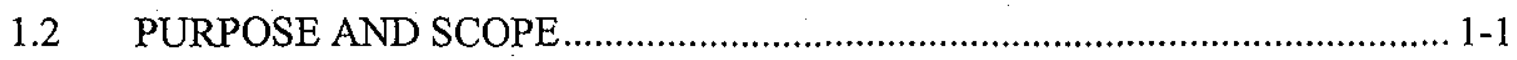

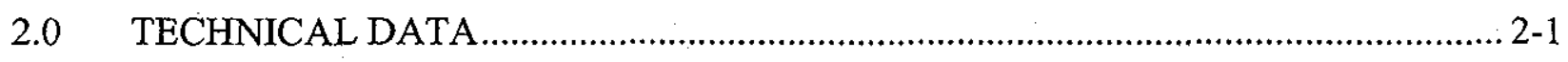

2.1 WTP SITE: SEISMIC ENTRY BOREHOLES …….................................... 2-1

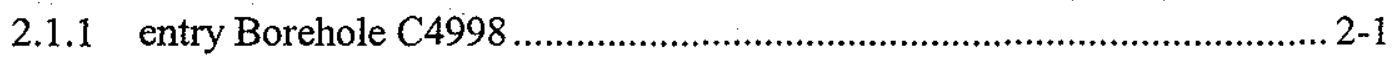

2.1.2 entry Borehole C4996 ....................................................................... 2-2

2.1.3 entry Borehole C4997 ....................................................................... 2-5

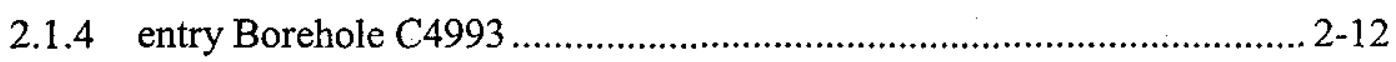

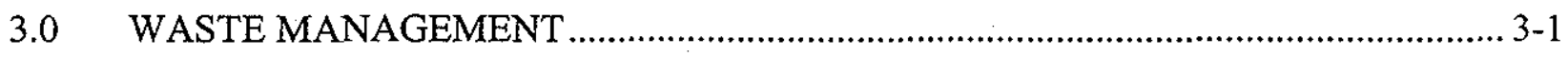

$3.1 \quad$ WTP SITE (200-PO-1 OU) ……............................................................... 3-1

3.1.1 Vadose Zone Cuttings....................................................................... 3-1

3.1.2 Saturated Zone Cuttings................................................................... $3-1$

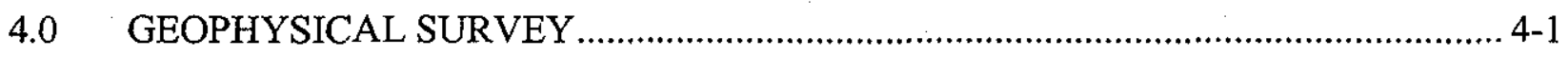

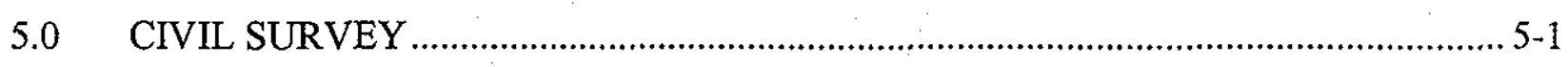

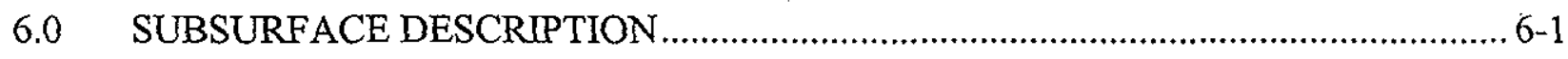

$6.1 \quad$ WTP SITE ............................................................................................. 6-1

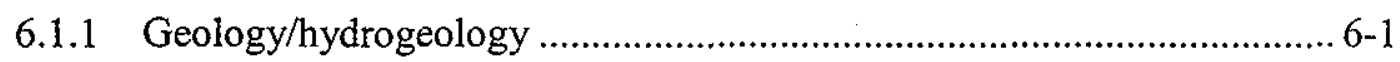

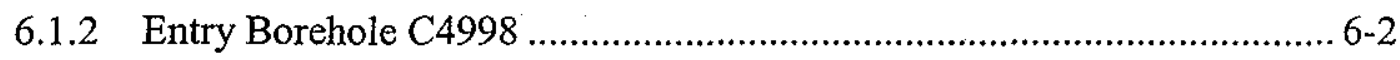

6.1.3 Entry Borehole C4996 ......................................................................... 6-2

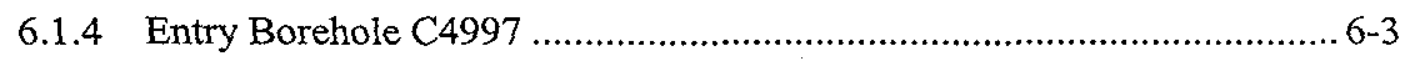

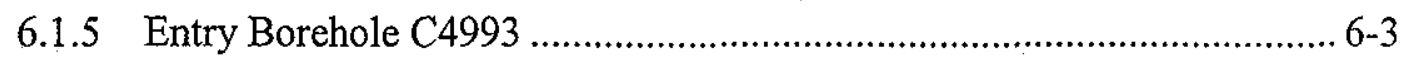

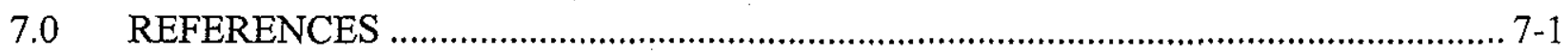




\section{FIGURES}

Figure 1-1. Location Map for Entry Boreholes Located within the WTP Site Boundary .......... 1-3

Figure 4-1: Geophysical Data for Seismic Entry Borehole C4998.

Figure 6-1: Subsurface Geology and Geophysical Data for Seismic Entry Borehole C4998 $6-4$

Figure 6-2: Subsurface Geology and Soil Sampling Intervals for Seismic Entry Borehole C4996

Figure 6-3: Subsurface Geology and Soil Sampling Intervals for Seismic Entry Borehole C4997 6-6

Figure 6-4: Subsurface Geology and Soil Sampling Intervals for Seismic Entry Borehole C4993 6-6

\section{TABLES}

Table 1-1. Drilling Summary of WTP Site Seismic Entry Boreholes $1-2$

Table 2-1. Construction Summary for Entry Borehole C4998. $2-2$

Table 2-2. Sediment Sampling Summary for Entry Borehole C4996 (2 pages) 2-3

Table 2-3. Construction Summary for Entry Borehole C4996. $2-5$

Table 2-4. Sediment Sampling Summary for Entry Borehole C4997 (6 pages) $.2-6$

Table 2-5. Water Sampling Summary for Entry Borehole C4997

Table 2-6. Construction Summary for Entry Borehole C4997. $2-12$

Table 2-7. Sediment Sampling Summary for Entry Borehole C4993 $2-14$

Table 2-8. Construction Summary for Entry Borehole C4993. $2-15$

Table 2-9. Suprabasalt Sedimentary Units Encountered at the WTP Entry Borehole Sites 


\section{WMP-32119, Rev. 0}

\section{APPENDICES}

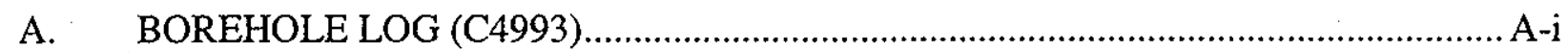

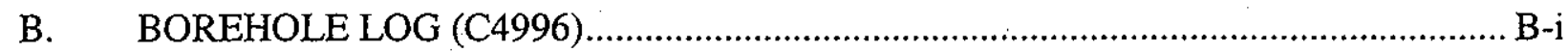

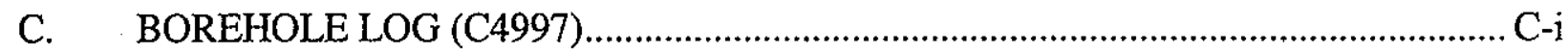

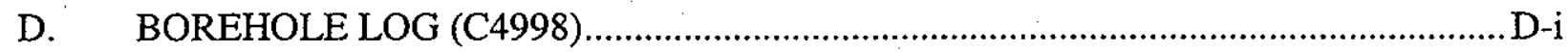

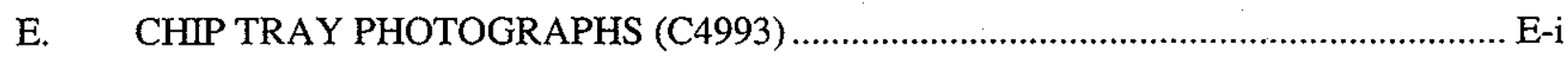

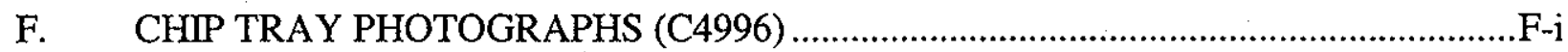

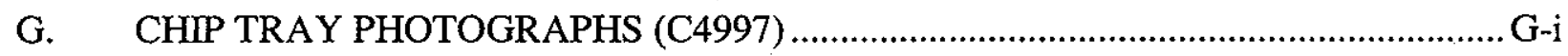

H. CHIP TRAY PHOTOGRAPHS (C4998) ................................................................. 
WMP-32119, Rev. 0

This page intentionally left blank. 
WMP-32119, Rev. 0

\section{ACRONYMS}

bgs

btoc

CERCLA

CFR

DOE-RL

DOW

DQO

EPA

FH

gpm

HWIS

ID

NMLS

NTU

OD

OU

PNNL

psi

SAP

SGLS

toc

$\mathrm{RCW}$

RLM

WAC below ground surface

below top of casing.

Comprehensive Environmental Response, Compensation, and Liability Act of 1980

Code of Federal Regulations

U.S. Department of Energy-Richland

Description of Work

Data Quality Objectives

U.S. Environmental Protection Agency

Fluor Hanford, Inc.

gallons per minute

Hanford Well Information System

inside diameter

Neutron Moisture Logging System

Nephelometric Turbidity Unit

outside diameter

Operable Unit

Pacific Northwest National Laboratory

pound per square inch

Sampling and Analysis Plan

Spectral Gamma Logging System

top of casing

Revised Code of Washington

Ringold Lower Mud

Washington Administrative Code 
WMP-32119, Rev. 0

This page intentionally left blank. 
WMP-32119, Rev. 0

\section{METRIC CONVERSION CHART}

\begin{tabular}{|c|c|c|c|c|c|}
\hline \multicolumn{3}{|c|}{ Into Metric Units } & \multicolumn{3}{|c|}{ Out of Metric Units } \\
\hline If You Know & Multiply By & To Get & If You Know & Multiply By & To Get \\
\hline Length & & & Length & & \\
\hline inches & 25.4 & millimeters & millimeters & 0.039 & inches \\
\hline inches & 2.54 & centimeters & centimeters & 0.394 & inches \\
\hline feet & 0.305 & meters & meters & 3.281 & feet \\
\hline yards & 0.914 & meters & meters & 1.094 & yards \\
\hline miles & 1.609 & kilometers & kilometers & 0.621 & miles \\
\hline Area & & & Area & & \\
\hline sq. inches & 6.452 & sq. centimeters & sq. centimeters & 0.155 & sq. inches \\
\hline sq. feet & 0.093 & sq. meters & sq. meters & 10.76 & sq. feet \\
\hline sq. yards & 0.0836 & sq. meters & sq. meters & 1.196 & sq. yards \\
\hline sq. miles & 2.6 & sq. kilometers & sq. kilometers & 0.4 & sq. miles \\
\hline acres & 0.405 & hectares & hectares & 2.47 & acres \\
\hline Mass (weight) & & & Mass (weight) & & \\
\hline ounces & 28.35 & grams & grams & 0.035 & ounces \\
\hline pounds & 0.454 & kilograms & kilograms & 2.205 & pounds \\
\hline ton & 0.907 & tonne & tonne & 1.102 & ton \\
\hline Volume & & & Volume & & \\
\hline teaspoons & 5 & milliliters & milliliters & 0.033 & fluid ounces \\
\hline tablespoons & 15 & milliliters & liters & 2.1 & pints \\
\hline fluid ounces & 30 & milliliters & liters & 1.057 & quarts \\
\hline cups & 0.24 & liters & liters & 0.264 & gallons \\
\hline pints & 0.47 & liters & cubic meters & 35.315 & cubic feet \\
\hline quarts & 0.95 & liters & cubic meters & 1.308 & cubic yards \\
\hline gallons & 3.8 & liters & & & \\
\hline cubic feet & 0.028 & cubic meters & & & \\
\hline cubic yards & 0.765 & cubic meters & & & \\
\hline Temperature & & & Temperature & & \\
\hline Fahrenheit & $\begin{array}{l}\text { subtract } 32 \\
\text { then multiply } \\
\text { by } 5 / 9\end{array}$ & Celsius & Celsius & $\begin{array}{l}\text { multiply by } \\
9 / 5, \text { then add } \\
32\end{array}$ & Fahrenheit \\
\hline Radioactivity & & & Radioactivity & & \\
\hline picocuries & 37 & millibecquerel & millibecquerel & 0.027 & picocuries \\
\hline
\end{tabular}


WMP-32119, Rev. 0

This page intentionally left blank. 
WMP-32119, Rev. 0

\subsection{INTRODUCTION}

This report describes the 2006 fiscal year field activities associated with the installation of four cable-tool-drilled boreholes located within the boundary of the Waste Treatment Plant (WTP), DOE Hanford site, Washington. The cable-tool-drilled boreholes extend from surface to $\sim 20 \mathrm{ft}$ below the top of basalt and were utilized as cased entry holes for three deep boreholes (approximately $1400 \mathrm{ft}$ ) that were drilled to support the acquisition of sub-surface geophysical data, and one deep corehole ( $1400 \mathrm{ft}$ ) that was drilled to acquire continuous core samples from underlying basalt and sedimentary interbeds. The geophysical data acquired from these boreholes will be integrated into a seismic response model that will provide the basis for defining the seismic design criteria for the WTP facilities.

These entry boreholes were installed for Pacific Northwest National Laboratory (PNNL) in accordance with the Drilling Plan for the Waste Treatment Plant Seismic Test Borehole Project (FS-RW-SWS-PN-005, Rev.0), and the Sampling and Analysis Plan (SAP): Waste Treatment Plant Seismic Boreholes Project (ICN PNNL-15848-1.1 and attached PNNL-15848, Rev. 2). Drilling data for these boreholes are summarized in Table 1-1. Documents supporting field activities as well as procedures followed during borehole characterization and construction are listed in Section 7.0 of this document.

\subsection{BACKGROUND}

Bechtel National, Inc., is under contract with the DOE to design and construct the WTP, located adjacent to the eastern edge of the 200 East Area of the DOE Hanford site, Washington. During the design/construction phase of the WTP, the data supporting the established seismic design criteria for the WTP was deemed insufficient. The DOE's office of River Protection requested that Battelle direct an extensive seismic investigation to obtain shear-wave seismic velocity data from the basalt and sedimentary interbeds that occur in the Saddle Mountains Basalt of the Columbia River Basalt Group (CRBG) that underlies the WTP site.

To improve the seismic response model for the WTP site, a network of three deep boreholes was selected to be drilled to provide access to the subsurface for collecting geophysical data, primarily shear wave (Vs) and compressional wave (Vp) and downhole geophysical logging data. These boreholes were also designed to provide samples to identify and characterize the geologic units that underlie the WTP site as well as provide sediment core samples (within the Hanford and Ringold Formations) for dynamic laboratory testing. In addition to these boreholes, a single wireline corehole was selected to be drilled to provide continuous core of the basalt and sedimentary interbeds to provide for correlation of the geology to the geophysical data.

\subsection{PURPOSE AND SCOPE}

The primary purpose of this field effort was to install four entry boreholes located within the boundary of the WTP site, east of the 200 East Area. These boreholes will provide cased entry holes for three deep boreholes and one deep corehole that will be used to acquire seismic data and continuous core from underlying basalt and sedimentary interbeds. The scope of activities described in this report includes the technical data that encompasses the drilling of four entry boreholes and related construction. Additional scope of work described in this report includes waste management and subsurface descriptions. All drilling data in this report are presented in 
the units in which they were measured in the field, with the exception of survey data where applicable which are reported in metric units. A summary of the cased boreholes is provided in Table 1-1 and the locations are shown in Figure 1-1.

Table 1-1. Drilling Summary of WTP Site Seismic Entry Boreholes

\begin{tabular}{|c|c|c|c|c|c|c|c|}
\hline \multirow{2}{*}{$\begin{array}{l}\text { Well } \\
\text { Name/Well } \\
\text { ID }\end{array}$} & \multirow{2}{*}{ Area } & \multicolumn{2}{|c|}{ Drilling Date } & \multirow{2}{*}{$\begin{array}{c}\text { Northing } \\
\text { (m) }\end{array}$} & \multirow{2}{*}{$\begin{array}{c}\text { Easting } \\
\text { (m) }\end{array}$} & \multirow{2}{*}{$\begin{array}{l}\text { Ground Surface } \\
\text { Elevation } \\
\text { (m) }\end{array}$} & \multirow{2}{*}{$\begin{array}{l}\text { Total } \\
\text { Entry } \\
\text { Depth } \\
\text { (feet bgs) }\end{array}$} \\
\hline & & Start & Finish & & & & \\
\hline C4993 & WTP Site & $8 / 21 / 06$ & 9/4/06 & 135756.7 & 576087.4 & NS & 383.5 \\
\hline C4996 & WTP Site & $7 / 12 / 06$ & $7 / 27 / 06$ & 136054.2 & 576145.2 & NS & 369 \\
\hline C4997 & WTP Site & $7 / 30 / 06$ & $8 / 18 / 06$ & 135781.0 & 576300.3 & NS & 401 \\
\hline C4998 & WTP Site & $6 / 12 / 06$ & $7 / 10 / 06$ & 135755.3 & 576309.4 & NS & 401.5 \\
\hline \multicolumn{8}{|c|}{$\begin{array}{l}\text { Notes: } \\
\text { Northing and easting coordinates are based on Washington State Plane Coordinates North American } \\
\text { Datum of } 1983 \text { (NAD83[91]) rounded to } 0.1 \mathrm{~m} \text {. } \\
\mathrm{ft} \text { bgs }=\text { feet below ground surface. } \\
\mathrm{NS}=\text { survey data not available at this time. }\end{array}$} \\
\hline
\end{tabular}


WMP-32119, Rev. 0

Figure 1-1. Location Map for Entry Boreholes Located within the WTP Site Boundary

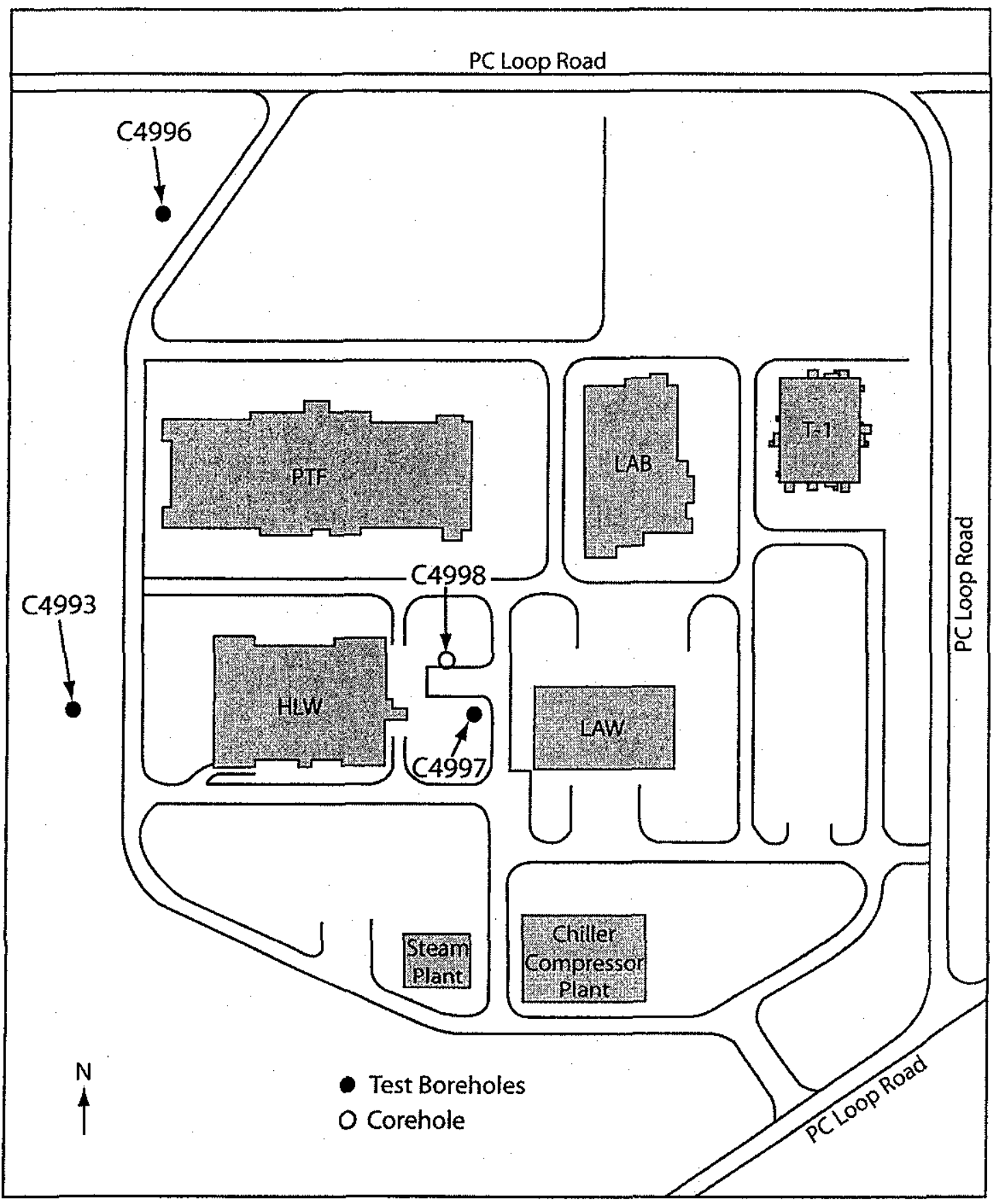


WMP-32119, Rev. 0

This page intentionally left blank. 
WMP-32119, Rev. 0

\subsection{TECHNICAL DATA}

This section provides technical details of the drilling methods, and completion during construction of the WTP seismic entry boreholes (see Figure 1-1). Drilling data are presented in Table 2-1 and geologic borehole logs are located in Appendices A through D.

\subsection{WTP SITE: SEISMIC ENTRY BOREHOLES}

\subsection{1 entry Borehole C4998}

This section summarizes activities related to the construction of entry borehole C4998.

\subsubsection{Drilling Summary}

Drilling of entry borehole C4998 began on June 12, 2006 using a cable tool drill rig, driving single wall carbon steel temporary casing with a $133 / 8$-inch outside diameter (OD) and $123 / 8$ inch inside diameter (ID) to a depth of $201.0 \mathrm{ft}$ below ground surface (bgs). Below $201.0 \mathrm{ft}$ bgs the casing was downsized to single wall carbon steel temporary casing with a $95 / 8$-inch OD and $85 / 8$-inch ID that was driven to a depth of $384.1 \mathrm{ft}$ bgs. The borehole was advanced using both core barrel and hard tool drilling methods to a total depth (TD) of $401.5 \mathrm{ft}$ bgs on July 10, 2006. The water table was initially encountered at approximately 278.1 feet bgs on June 30, 2006.

\subsubsection{Sample Summary}

Archive samples (1 -pint glass jars) were collected for FH and PNNL at five-ft intervals, but were not analyzed in the field. In addition to archive samples, lithologic changes were recorded and collected in plastic chip trays for future characterization use by both FH and PNNL.

\subsubsection{Construction Summary}

Entry Borehole construction for borehole C4998 was designed to enable deep wire-line coring operations to advance the borehole to approximately $1300 \mathrm{ft}$ to $1500 \mathrm{ft}$ bgs. Construction materials and formation seal intervals are discussed below. Borehole plumbness testing was performed at approximately 50 -ft intervals during borehole advancement using an $103 / 4$-inch $\mathrm{OD}, 20$-ft long tool within the $133 / 8$-inch OD casing and a $65 / 8$-inch $\mathrm{OD}, 20$-ft long tool within the $95 / 8$-inch OD casing. Additional plumbness testing was performed at approximately $50-\mathrm{ft}$ intervals using a gyro survey tool. A separate report will provide specific details of these gyro surveys. Construction and completion of this entry borehole was carried out from July 11, 2006 to July 12,2006 . Entry borehole construction summary data are provided in Table 2-1.

- Construction materials and grout seal interval. To support wire-line coring operations, $403.0 \mathrm{ft}$ of $4 \frac{1}{2}$-inch ID carbon steel temporary casing with centralizers placed approximately every $40 \mathrm{ft}$ were installed from $3.9 \mathrm{ft}$ ags to $399.1 \mathrm{ft}$ bgs (1.4 ft off bottom). A grout seal was installed from $348.5 \mathrm{ft}$ to $401.5 \mathrm{ft}$ bgs, consisting of Portland Type I/II cement and 5\% bentonite powder by volume per WAC 173-160. After the cement grout was in place the $4 \frac{1}{2}$-inch temporary casing was lowered to $401.2 \mathrm{ft}$ bgs. 
WMP-32119, Rev. 0, RE-ISSUE

Table 2-1. Construction Summary for Entry Borehole C4998

\begin{tabular}{|c|c|c|c|c|c|c|c|}
\hline Borehole ID & $\begin{array}{l}\text { Borehole } \\
\text { Location }\end{array}$ & $\begin{array}{l}\text { Total } \\
\text { Depth } \\
\text { (ft bgs) }\end{array}$ & $\begin{array}{l}\text { Water Level } \\
\text { (ft bgs) }\end{array}$ & $\begin{array}{l}133 / 8 \text { in. OD } \\
\text { casing depth }\end{array}$ & $\begin{array}{l}95 / 8 \text { in. OD } \\
\text { casing depth }\end{array}$ & $\begin{array}{l}41 / 2 \text { in. OD } \\
\text { casing depth }\end{array}$ & $\begin{array}{l}\text { Grout Seal } \\
\text { Interval }\end{array}$ \\
\hline C4998 & WTP Site & 401.5 & 278.1 & 201.0 & 384.5 & 401.2 & $348.5-401.5$ \\
\hline $\begin{array}{l}\text { Notes: } \\
\mathrm{ft} \text { bgs }=\text { fe } \\
\text { in. = inch } \\
\mathrm{OD}=\text { outs }\end{array}$ & $10 \mathrm{Wr}$ & surface. & & & 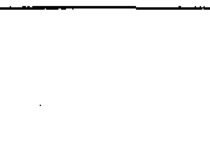 & & \\
\hline
\end{tabular}

\subsubsection{Entry Borehole C4996}

This section summarizes activities related to the construction of entry borehole C4996.

\subsubsection{Drilling Summary}

Drilling of entry borehole C4996 began on July 12, 2006 using a cable tool drill rig, driving single wall carbon steel temporary casing with a $133 / 8$ inch OD and $123 / 8$ inch ID to a depth of $211.3 \mathrm{ft}$ below ground surface (bgs). Below $211.3 \mathrm{ft}$ bgs the casing was downsized to single wall carbon steel temporary casing with a $95 / 8$ inch OD and $85 / 8$ inch ID that was driven to a depth of $350.0 \mathrm{ft}$ bgs. The borehole was advanced using both core barrel and hard tool drilling methods to a total depth of $369.0 \mathrm{ft}$ bgs on July 27,2006 . The water table was initially encountered at approximately 270.3 feet bgs on July 23, 2006.

\subsubsection{Sample Summary}

Field sampling data are presented in Table 2.2. Six split spoon samples and six corresponding drive barrel samples were collected at discrete intervals and will be used in a laboratory testing program to obtain site-specific soil property data. Soil testing procedures are detailed in ASTM D4015-92 (Standard Test Methods for Modulus and Damping of Soils by the Resonant-Column Method) and GR06-4 (Test Procedures and Calibration Documentation Associated with the RCTS and URC Tests at the University of Texas at Austin). Additional soil samples (1-pint glass jars) were collected directly above and below each split spoon interval and will be used in laboratory testing for tritium analysis. Archive samples (1-pint glass jars) were collected for $\mathrm{FH}$ and PNNL, at five-ft intervals, but were not analyzed in the field. In addition to archive samples, lithologic changes were recorded and collected in plastic chip trays for future characterization use by both FH and PNNL. Table 2-2 summarizes the field sampling data for entry borehole C4996. 
WMP-32119, Rev. 0

Table 2-2. Sediment Sampling Summary for Entry Borehole C4996 (2 pages)

\begin{tabular}{|c|c|c|c|c|c|c|c|c|c|c|c|}
\hline $\begin{array}{l}\text { Date } \\
(\mathbf{2 0 0 6 )}\end{array}$ & $\begin{array}{l}\text { Sample } \\
\text { Type }\end{array}$ & $\begin{array}{c}\text { Depth Intv. } \\
\text { (ft bgs) }\end{array}$ & $\begin{array}{c}\text { HEIS } \\
\text { Number }\end{array}$ & Liner & $\begin{array}{l}\text { Liner } \\
\text { Depth }\end{array}$ & \multicolumn{2}{|c|}{$\begin{array}{l}\text { Blow } \\
\text { Counts }\end{array}$} & \multicolumn{2}{|c|}{$\begin{array}{c}\% \\
\text { Rec. }\end{array}$} & \multicolumn{2}{|c|}{ Strat. } \\
\hline \multirow{7}{*}{ 17-JuI } & Tritium & 187.0 & \multicolumn{7}{|c|}{$\mathrm{N} / \mathrm{A}$} & \multirow{7}{*}{ 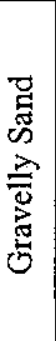 } & \\
\hline & \multirow{4}{*}{ Split Spoon } & \multirow{4}{*}{$187.5-190.0$} & N/A & Shoe & $189.0-190.0$ & \multirow{4}{*}{186} & 53 & \multicolumn{2}{|c|}{ ND } & & \\
\hline & & & $\mathrm{B} 1 \mathrm{~K} 4 \mathrm{Y} 1$ & $\mathrm{~A}$ & $188.5-189.0$ & & 7 & \multirow{3}{*}{60} & $\mathrm{ND}$ & & \\
\hline & & & $\mathrm{B} 1 \mathrm{~K} 4 \mathrm{Y} 2$ & $\mathrm{~B}$ & $188.0-188.5$ & & 31 & & ND & & $\dot{\Xi}$ \\
\hline & & & B1K4Y3 & $\mathrm{C}$ & $187.5-188.0$ & & 65 & & ND & & \\
\hline & Drive Barrel & $187.5-190.0$ & B1K4Y5 & \multicolumn{4}{|c|}{ N/A } & \multirow{2}{*}{\multicolumn{2}{|c|}{$\mathrm{ND}$}} & & \\
\hline & Tritium & 190.0 & \multicolumn{5}{|c|}{ N/A } & & & & \\
\hline \multirow{6}{*}{ 20-Jul } & \multirow{5}{*}{ Split Spoon } & \multirow{5}{*}{$230.5-233$} & N/A & Shoe & $232.5-233.0$ & \multirow{5}{*}{269} & 49 & \multicolumn{2}{|c|}{0} & \multirow{6}{*}{ 岕 } & \\
\hline & & & B1K4Y6 & $\mathrm{A}$ & $232.0-232.5$ & & 50 & & ND & & \\
\hline & & & B1K4Y7 & $\mathrm{B}$ & $231.5-232.0$ & & 70 & & $\mathrm{ND}$ & & \\
\hline & & & $\mathrm{B} 1 \mathrm{~K} 4 \mathrm{Y} 8$ & $\mathrm{C}$ & $231.0-231.5$ & & 82 & 95 & $\mathrm{ND}$ & & \\
\hline & & & $\mathrm{B} 1 \mathrm{~K} 4 \mathrm{Y} 9$ & $\mathrm{D}$ & $230.5-231.0$ & & 18 & & ND & & \\
\hline & Drive Barrel & $187.5-190.0$ & B1K4Y5 & \multicolumn{4}{|c|}{ N/A } & \multicolumn{2}{|c|}{$\mathrm{ND}$} & & \\
\hline \multirow{8}{*}{ 20-Jul } & Tritium & 252.0 & \multicolumn{7}{|c|}{ N/A } & & \\
\hline & \multirow{5}{*}{ Split Spoon } & \multirow{5}{*}{$252.0-254.5$} & N/A & Shoe & $254.0-254.5$ & \multirow{5}{*}{295} & 20 & \multicolumn{2}{|c|}{ ND } & & \\
\hline & & & $\mathrm{B} 1 \mathrm{~K} 501$ & $\mathrm{~A}$ & $25305-254.0$ & & 71 & & 33 & $\bar{\Phi}$ & \\
\hline & & & $\mathrm{B} 1 \mathrm{~K} 502$ & B & $253.0-25305$ & & 116 & 80 & 100 & 造 & (2) \\
\hline & & & B1K503 & $\mathrm{C}$ & $252.5-253.0$ & & 68 & 80 & 100 & 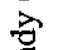 & \\
\hline & & & B1K504 & $\mathrm{D}$ & $252.0-252.5$ & & 20 & & 83 & 氶 & \\
\hline & Drive Barrel & $252.0-254.0$ & B1K506 & & N/A & & & & $\mathrm{D}$ & & \\
\hline & Tritium & 254.5 & & & $\mathrm{~N} / \mathrm{A}$ & & & & & & \\
\hline & Tritium & 275.5 & & & N/A & & & & & & \\
\hline & & & $\mathrm{N} / \mathrm{A}$ & Shoe & $277.5-278.2$ & & 60 & & 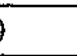 & & \\
\hline & & & B1K505 & $\mathrm{A}$ & $277.0-277.5$ & & 65 & & 100 & & \\
\hline $21 \mathrm{~J} 1$ & Split Spoon & $275.5-278.2$ & $\mathrm{~B} 1 \mathrm{~K} 507$ & $B$ & $276.5-277.0$ & 250 & 60 & 05 & 100 & $\overrightarrow{0}$ & \\
\hline $21-\mathrm{Ju}$ & & & $\mathrm{B} 1 \mathrm{~K} 508$ & $\mathrm{C}$ & $276.0-276.5$ & & 49 & 95 & 100 & 䞤 & \\
\hline & & & B1K509 & $\mathrm{D}$ & $275.5-276.0$ & & 16 & & 90 & & \\
\hline & Drive Barrel & $275.5-278.0$ & $\mathrm{~B} 1 \mathrm{~K} 510$ & N/A & N/A & $\mathrm{N} /$ & & & $\mathrm{D}$ & & \\
\hline & Tritium & 254.5 & N/A & $\mathrm{N} / \mathrm{A}$ & $\mathrm{N} / \mathrm{A}$ & $\mathrm{N} /$ & & & A & & \\
\hline
\end{tabular}


Table 2-2. Sediment Sampling Summary for Entry Borehole C4996 (2 pages)

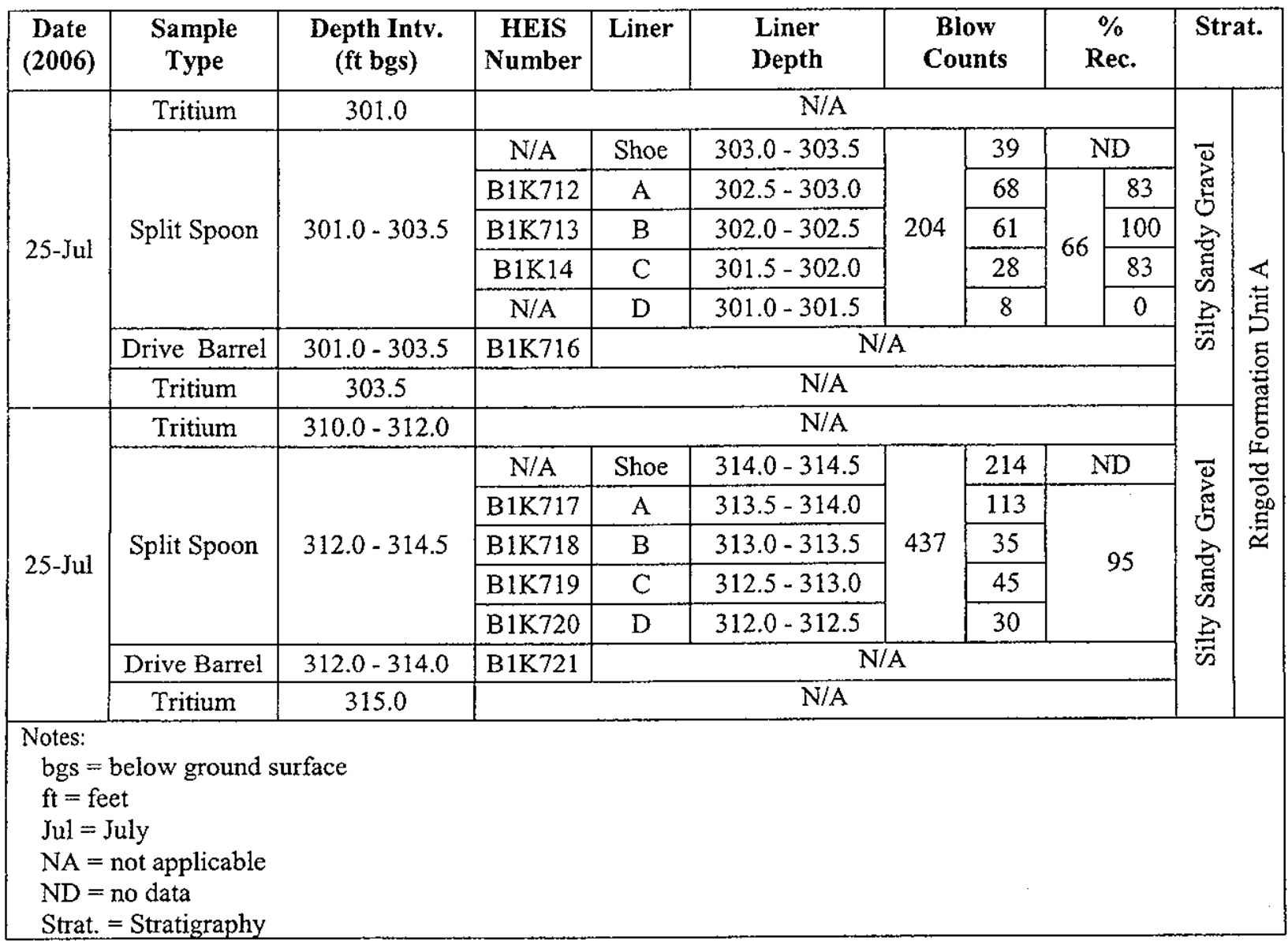

\subsubsection{Construction Summary}

Entry Borehole construction for borehole C4996 was designed to enable deep mud-rotary drilling to advance the borehole to approximately $1300 \mathrm{ft}$ to $1500 \mathrm{ft}$ bgs. After a total depth of $369.0 \mathrm{ft}$ bgs ( $20 \mathrm{ft}$ into basalt) was obtained, a grout seal was installed from about $330 \mathrm{ft}$ to $369 \mathrm{ft}$ bgs, consisting of Portland Type $/ / \mathrm{II}$ cement and $5 \%$ bentonite powder by volume per WAC 173-160. After the cement grout was in place the $95 / 8$-inch temporary casing was backpulled $5 \mathrm{ft}$ to allow the cement to make contact with the formation and then driven back to $350 \mathrm{ft}$ bgs. Borehole plumbness testing was performed at approximately 50 - $\mathrm{ft}$ intervals during borehole advancement using a $103 / 4$-inch $\mathrm{OD}, 20$-ft long tool within the 13 3/8-inch OD casing and a $65 / 8$-inch OD, 20-ft long tool within the $95 / 8$-inch OD casing. Additional plumbness testing was performed at approximately 50 -ft intervals using a gyro survey tool. A separate report will provide specific details of these gyro surveys. Construction and completion of this entry borehole was carried out on July 27, 2006. Entry borehole construction summary data are provided in Table 2-3. 
Table 2-3. Construction Summary for Entry Borehole C4996

\begin{tabular}{|c|c|c|c|c|c|c|}
\hline $\begin{array}{l}\text { Borehole } \\
\text { ID }\end{array}$ & $\begin{array}{l}\text { Borehole } \\
\text { Location }\end{array}$ & $\begin{array}{l}\text { Total } \\
\text { Depth } \\
\text { (ft bgs) }\end{array}$ & $\begin{array}{l}\text { Water Level } \\
\text { (ft bgs) }\end{array}$ & $\begin{array}{l}133 / 8 \text { in. } O D \\
\text { casing depth }\end{array}$ & $\begin{array}{l}95 / 8 \text { in. } O D \\
\text { casing depth }\end{array}$ & $\begin{array}{l}\text { Grout Seal } \\
\text { Interval }\end{array}$ \\
\hline $\mathrm{C} 4996$ & WTP Site & 369.0 & 270.3 & 211.3 & 350.0 & $330.4-369.0$ \\
\hline \multicolumn{7}{|c|}{$\begin{array}{l}\text { Notes: } \\
\mathrm{ft} \text { bgs }=\text { feet below ground surface. } \\
\text { in. = inch } \\
\mathrm{OD}=\text { outside diameter. }\end{array}$} \\
\hline
\end{tabular}

\subsubsection{Entry Borehole C4997}

This section summarizes activities related to the construction of entry borehole C4997.

\subsubsection{Drilling Summary}

Drilling of entry borehole C4997 began on July 30, 2006 using a cable tool drill rig, driving single wall carbon steel temporary casing with a 13 3/8-inch OD and $123 / 8$-inch ID to a depth of $216.5 \mathrm{ft}$ bgs. Below $216.5 \mathrm{ft}$ bgs the casing was downsized to single wall carbon steel temporary casing with a $95 / 8$ inch OD and $85 / 8$ inch $\mathrm{ID}$ that was driven to $383.3 \mathrm{ft}$ bgs. The borehole was advanced using both core barrel and hard tool drilling methods to a total depth of $401.0 \mathrm{ft}$ bgs on August 18,2006 . The water table was initially encountered at approximately 277.5 feet bgs on August 9, 2006.

\subsubsection{Sample Summary}

Field sampling data are presented in Table 2.4. Several split spoon samples and corresponding drive barrel samples were collected at discrete intervals and will be used in a laboratory testing program to obtain site-specific soil property data. Soil testing procedures are detailed in ASTM D4015-92 (Standard Test Methods for Modulus and Damping of Soils by the Resonant-Column Method) and GR06-4 (Test Procedures and Calibration Documentation Associated with the RCTS and URC Tests at the University of Texas at Austin). Soil samples (1-pint glass jars) were collected directly above and below each split spoon interval and will be used in laboratory testing for tritium analysis. Additional soil samples (1-pint glass jars) were collected at each split spoon interval and will be used for particle size analysis (ASTM D6913-04e1). Archive samples (1-pint glass jars) were collected for FH and PNNL, at five-ft intervals, but were not analyzed in the field. In addition to the archive samples, lithologic changes were recorded and collected in plastic chip trays for future characterization use by both FH and PNNL. Several water samples were collected via kabis sampler from four isolated depth intervals and will be tested for various chemical properties by FH. Table 2-4 summarizes the sediment sampling data and Table 2-5 summarizes the water sampling data for borehole C4997. 
WMP-32119, Rev. 0

Table 2-4. Sediment Sampling Summary for Entry Borehole C4997 (6 pages)

\begin{tabular}{|c|c|c|c|c|c|c|c|c|c|c|}
\hline $\begin{array}{c}\text { Date } \\
(2006)\end{array}$ & $\begin{array}{c}\text { Sample } \\
\text { Type }\end{array}$ & $\begin{array}{l}\text { Depth Intv. } \\
\text { (ft bgs) }\end{array}$ & $\begin{array}{c}\text { HEIS } \\
\text { Number }\end{array}$ & Liner & $\begin{array}{l}\text { Liner } \\
\text { Depth }\end{array}$ & \multicolumn{2}{|c|}{$\begin{array}{l}\text { Blow } \\
\text { Counts }\end{array}$} & $\begin{array}{l}\% \\
\text { Rec. }\end{array}$ & \multicolumn{2}{|c|}{ Strat. } \\
\hline \multirow{6}{*}{ 30-Jul } & \multirow{5}{*}{ Split Spoon } & \multirow{5}{*}{$20.6-23.1$} & N/A & Shoe & $22.6-32.1$ & \multirow{5}{*}{192} & 47 & \multirow{5}{*}{100} & \multirow{6}{*}{ 莺 } & \\
\hline & & & $\mathrm{B} 1 \mathrm{~K} 867$ & A & $20.6-21.1$ & & 25 & & & \\
\hline & & & $\mathrm{B} 1 \mathrm{~K} 868$ & B & $21.1-21.6$ & & 35 & & & \\
\hline & & & $\mathrm{B} 1 \mathrm{~K} 869$ & $\mathrm{C}$ & $21.6-22.1$ & & 40 & & & \\
\hline & & & $\mathrm{B} 1 \mathrm{~K} 870$ & $\mathrm{D}$ & $22.1-22.6$ & & 45 & & & \\
\hline & Drive Barrel & $20.6-23.1$ & $\mathrm{~B} 1 \mathrm{~K} 8 \mathrm{C} 5$ & \multicolumn{5}{|c|}{ N/A } & & \\
\hline \multirow{7}{*}{ 31-Jul. } & \multirow{5}{*}{ Split Spoon } & \multirow{5}{*}{$30.1-32.6$} & $\mathrm{~N} / \mathrm{A}$ & Shoe & $32.1-32.6$ & \multirow{5}{*}{156} & 33 & \multirow{5}{*}{100} & \multirow{7}{*}{ 胥 } & \multirow{32}{*}{ 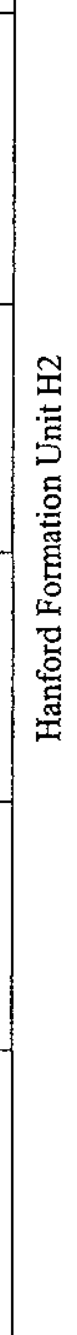 } \\
\hline & & & $\mathrm{B} 1 \mathrm{~K} 871$ & $\mathrm{~A}$ & $31.6-32.1$ & & 39 & & & \\
\hline & & & B1K871 & B & $31.1-31.6$ & & 45 & & & \\
\hline & & & B1K871 & $\mathrm{C}$ & $30.6-31.1$ & & 29 & & & \\
\hline & & & B1K871 & $\mathrm{D}$ & $30.1-30.6$ & & 10 & & & \\
\hline & Drive Barrel & $30.1-32.1$ & ND & & $\mathrm{N} / \mathrm{A}$ & & & & & \\
\hline & P.S. & 30.5 & & & N/A & & & & & \\
\hline \multirow{6}{*}{ 31-Jul } & \multirow{5}{*}{ Split Spoon } & \multirow{5}{*}{$39.4-41.9$} & N/A & Shoe & $41.4-41.9$ & \multirow{5}{*}{302} & 89 & \multirow{5}{*}{100} & \multirow{6}{*}{ 莺 } & \\
\hline & & & B1K875 & $\mathrm{A}$ & $40.9-41.4$ & & 63 & & & \\
\hline & & & $\mathrm{B} 1 \mathrm{~K} 876$ & $B$ & $40.4-40.9$ & & 65 & & & \\
\hline & & & B1K 877 & $\mathrm{C}$ & $39.9-40.4$ & & 65 & & & \\
\hline & & & B1K878 & $\mathrm{D}$ & $39.4-39.9$ & & 20 & & & \\
\hline & P. S. & 40.0 & & & N/A & & & & & \\
\hline & & & N/A & Shoe & $52.2-52.7$ & & 134 & & & \\
\hline & & & B1K879 & $\mathrm{A}$ & $51.7-52.2$ & & 69 & & & \\
\hline & Split Spoon & $50.2-52.7$ & B1K880 & B & $51.2-51.7$ & 308 & 58 & 100 & D्g & \\
\hline 31-Jul & & & B1K881 & $\mathrm{C}$ & $50.7-51.2$ & & 38 & & 袄 & \\
\hline & & & B1K882 & $\mathrm{D}$ & $50.2-50.7$ & & 9 & & & \\
\hline & P.S. & 50.5 & & & N/A & & & & & \\
\hline & & & N/A & Shoe & $62.3-62.8$ & & 134 & & & \\
\hline & & & $\mathrm{B} 1 \mathrm{~K} 883$ & $\mathrm{~A}$ & $61.8-62.3$ & & 55 & & & \\
\hline & Split Spoon & $60.3-62.8$ & B1K884 & B & $61.3-61.8$ & 294 & 60 & 100 & Z & \\
\hline $31-J u l$ & & & B1K 885 & $\mathrm{C}$ & $60.8-61.3$ & & 25 & & 氙 & \\
\hline & & & B1K 886 & $\mathrm{D}$ & $60.3-60.8$ & & 20 & & & \\
\hline & P.S. & 60.0 & & & N/A & & & & & \\
\hline & & & N/A & Shoe & $71.8-72.3$ & & 111 & & & \\
\hline & & & BIK 887 & $A$ & $71.3-71.8$ & & 52 & & & \\
\hline & Split Spoon & $69.8-72.3$ & B1K888 & $\mathrm{B}$ & $70.8-71.3$ & 228 & 36 & 100 & & \\
\hline 1-Aug & & & B1K 889 & $\mathrm{C}$ & $70.3-70.8$ & & 20 & & 荡 & \\
\hline & & & B1K890 & $\mathrm{D}$ & $69.8-70.3$ & & 9 & & & \\
\hline & Drive Barrel & $70.0-72.5$ & $\mathrm{~B} 1 \mathrm{~K} 8 \mathrm{C} 6$ & & $\mathrm{~N} / \mathrm{A}$ & & & & & \\
\hline & P.S. & 70.0 & & & $\mathrm{~N} / \mathrm{A}$ & & & & & \\
\hline
\end{tabular}


WMP-32119, Rev. 0

Table 2-4. Sediment Sampling Summary for Entry Borehole C4997 (6 pages)

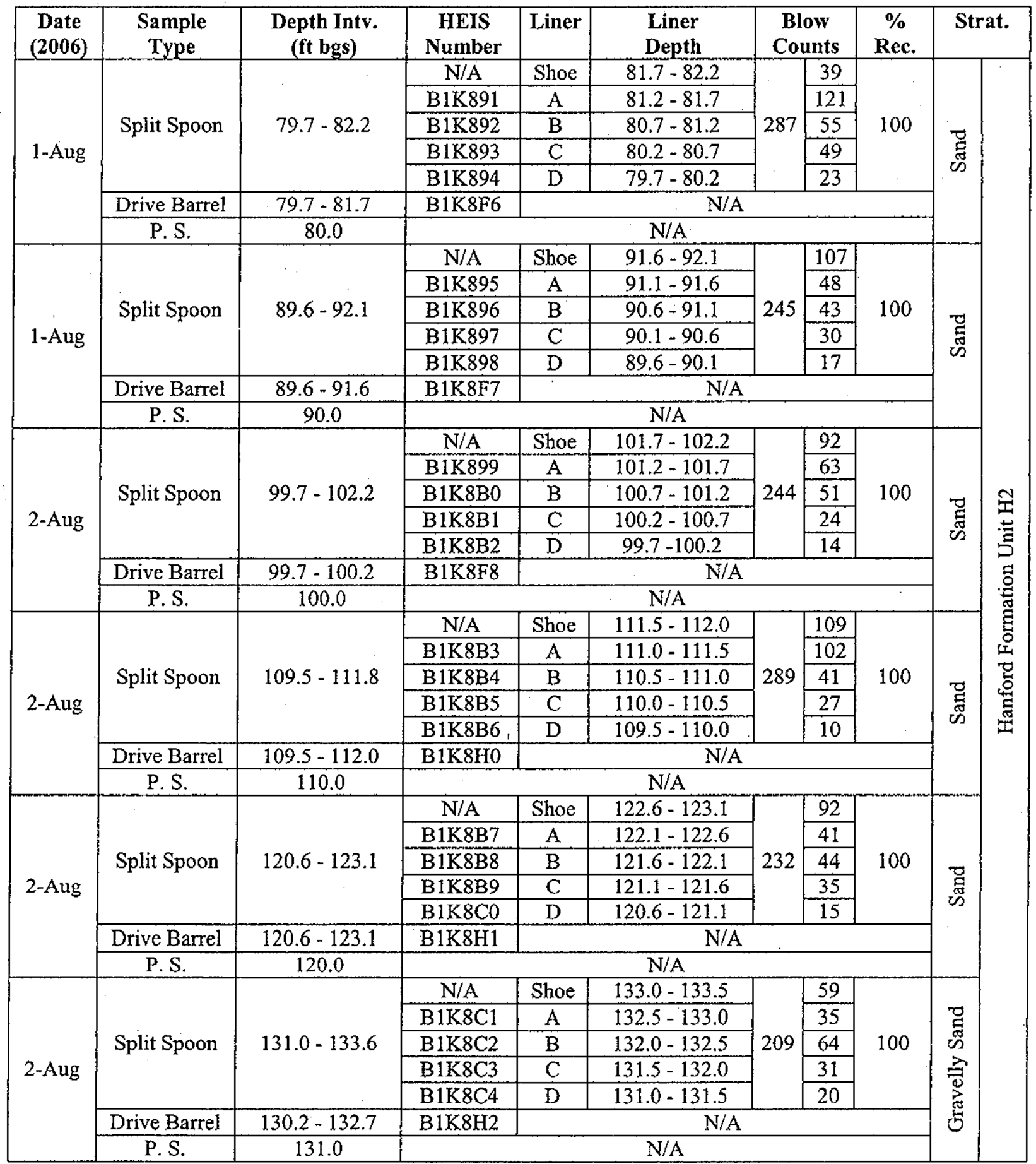


WMP-32119, Rev. 0

Table 2-4. Sediment Sampling Summary for Entry Borehole C4997 (6 pages)

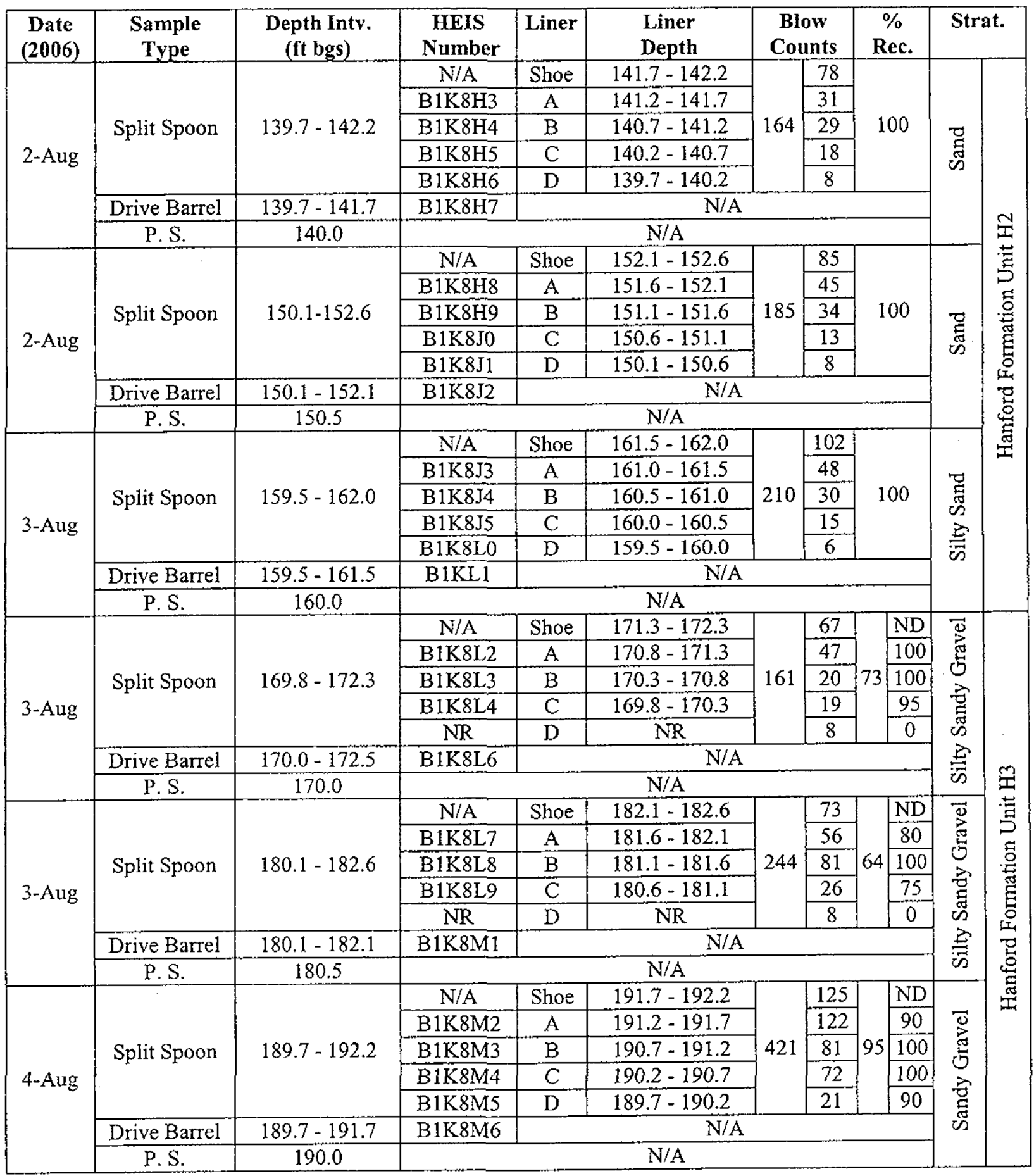


WMP-32119, Rev. 0

Table 2-4. Sediment Sampling Summary for Entry Borehole C4997 (6 pages)

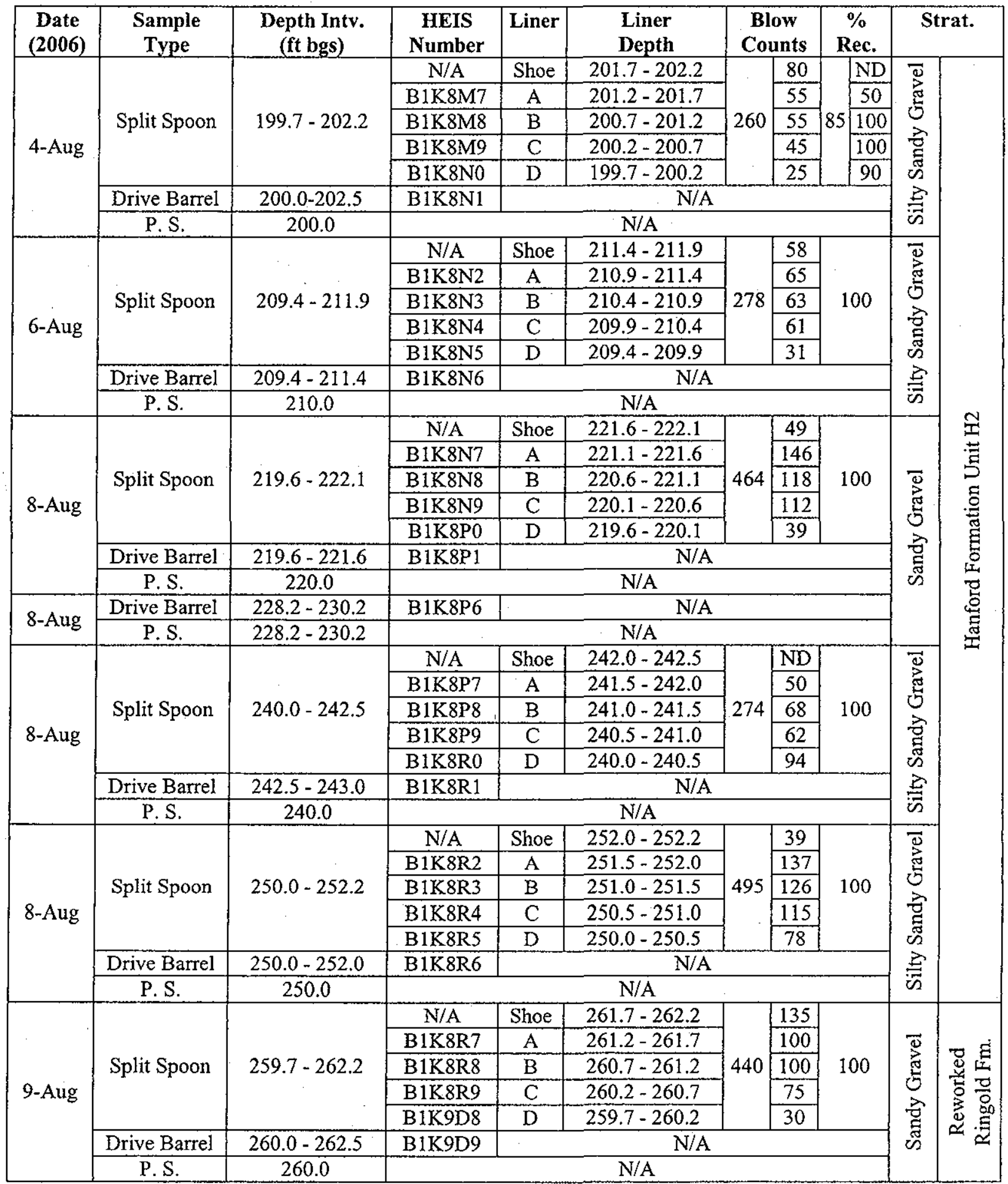


WMP-32119, Rev. 0

Table 2-4. Sediment Sampling Summary for Entry Borehole C4997 (6 pages)

\begin{tabular}{|c|c|c|c|c|c|c|c|c|c|c|}
\hline $\begin{array}{l}\text { Date } \\
(2006)\end{array}$ & $\begin{array}{c}\text { Sample } \\
\text { Type }\end{array}$ & $\begin{array}{l}\text { Depth Intv. } \\
\text { (ft bgs) }\end{array}$ & $\begin{array}{c}\text { HEIS } \\
\text { Number }\end{array}$ & Liner & $\begin{array}{l}\text { Liner } \\
\text { Depth }\end{array}$ & \multicolumn{2}{|c|}{$\begin{array}{c}\text { Blow } \\
\text { Counts }\end{array}$} & $\begin{array}{c}\% \\
\text { Rec. }\end{array}$ & \multicolumn{2}{|c|}{ Strat. } \\
\hline \multirow{7}{*}{ 9-Aug } & \multirow{5}{*}{ Split Spoon } & \multirow{5}{*}{$269.4-272.0$} & N/A & Shoe & $271.4-272.0$ & \multirow{5}{*}{339} & 124 & \multirow{5}{*}{100} & \multirow{7}{*}{ 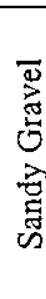 } & \\
\hline & & & B1K9F0 & A & $270.9-271.4$ & & 73 & & & \\
\hline & & & B1K9F1 & B & $270.4-270.9$ & & 66 & & & \\
\hline & & & $\mathrm{B} 1 \mathrm{~K} 9 \mathrm{~F} 2$ & $\mathrm{C}$ & $269.9-270.4$ & & 48 & & & \\
\hline & & & $\mathrm{B} 1 \mathrm{~K} 9 \mathrm{~F} 3$ & $\mathrm{D}$ & $269.4-269.9$ & & 18 & & & \\
\hline & Drive Barrel & $270.0-272.5$ & B1K9F4 & \multicolumn{5}{|c|}{ N/A } & & \\
\hline & P.S. & 270.0 & \multicolumn{6}{|c|}{ N/A } & & \\
\hline \multirow{8}{*}{ 9-Aug } & Tritium/P.S. & 280.0 & & & N/A & & & & \multirow{8}{*}{ 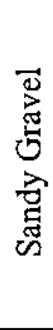 } & \\
\hline & \multirow{5}{*}{ Split Spoon } & \multirow{5}{*}{$280.0-282.5$} & N/A & Shoe & $282.0-282.5$ & \multirow{5}{*}{586} & 39 & \multirow{5}{*}{100} & & \\
\hline & & & B1K9B8 & $\mathrm{A}$ & $281.5-282.0$ & & 146 & & & \\
\hline & & & B1K9B9 & $\mathrm{B}$ & $281.0-281.5$ & & 205 & & & \\
\hline & & & $\mathrm{B} 1 \mathrm{~K} 9 \mathrm{C} 0$ & $\mathrm{C}$ & $280.5-281.0$ & & 132 & & & \\
\hline & & & $\mathrm{B} 1 \mathrm{~K} 9 \mathrm{C} 1$ & $\mathrm{D}$ & $280.0-280.5$ & & 64 & & & \\
\hline & Drive Barrel & $280.0-282.0$ & $\mathrm{~B} 1 \mathrm{~K} 9 \mathrm{~F} 5$ & \multicolumn{5}{|c|}{ N/A } & & \\
\hline & Tritium & 283.0 & \multicolumn{6}{|c|}{ N/A } & & \\
\hline \multirow{8}{*}{ 10-Aug } & Tritium/P.S. & 293.0 & & & $\mathrm{~N} / \mathrm{A}$ & & & & \multirow{8}{*}{ 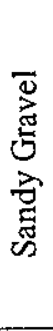 } & \\
\hline & \multirow{5}{*}{ Split Spoon } & & $\mathrm{N} / \mathrm{A}$ & Shoe & $294.6-295.1$ & \multirow{5}{*}{225} & 69 & & & \\
\hline & & & B1K9M8 & $\mathrm{A}$ & $294.1-294.6$ & & 58 & & & : ت્ه \\
\hline & & $292.6-295.1$ & B1K9M9 & B & $293.6-294.1$ & & 49 & 100 & & 丞 \\
\hline & & & B1K9N0 & $\mathrm{C}$ & $293.1-293.6$ & & 34 & & & î \\
\hline & & & $\mathrm{B} 1 \mathrm{~K} 9 \mathrm{~N} 1$ & $\mathrm{D}$ & $292.6-293.1$ & & 15 & & & 믕 \\
\hline & Drive Barrel & $292.6-294.6$ & $\mathrm{~B} 1 \mathrm{~K} 9 \mathrm{~N} 2$ & & $\mathrm{~N} / \mathrm{A}$ & & & & & 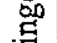 \\
\hline & Tritium & 295.0 & & & N/A & & & & & $\approx$ \\
\hline & Tritium/P.S. & 300.0 & & & N/A & & & & & g్ \\
\hline & & & N/A & Shoe & $302.0-302.5$ & & 36 & $\mathrm{ND}$ & & 管 \\
\hline & & & $\begin{array}{l}\mathrm{B} 1 \mathrm{~K} 9 \mathrm{C} 2 \\
\mathrm{~B} 1 \mathrm{~K} 9 \mathrm{C} 6\end{array}$ & A & $301.5-302.0$ & & 20 & 50 & & 急 \\
\hline 10. & $\begin{array}{c}\text { Split Spoon } \\
\text { (FH VOA }\end{array}$ & $300.0-302.5$ & $\begin{array}{l}\mathrm{B} 1 \mathrm{~K} 9 \mathrm{C} 3 \\
\mathrm{~B} 1 \mathrm{~K} 9 \mathrm{C} 7\end{array}$ & B & $301.0-301.5$ & 159 & 15 & 90100 & 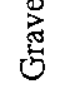 & \\
\hline 10-Aug & Samples) & & $\begin{array}{l}\mathrm{B} 1 \mathrm{~K} 9 \mathrm{C} 4 \\
\mathrm{~B} 1 \mathrm{~K} 9 \mathrm{C} 8\end{array}$ & $\mathrm{C}$ & $300.5-301.0$ & & 62 & 100 & 总 & \\
\hline & & & $\begin{array}{l}\text { B1K9C5 } \\
\text { B1K9C9 }\end{array}$ & D & $300.0-300.5$ & & 26 & 100 & & \\
\hline & Drive Barrel & $300.0-302.0$ & $\mathrm{~B} 1 \mathrm{~K} 9 \mathrm{~N} 2$ & & N/A & & & & & \\
\hline & Tritium & 303.0 & & & N/A & & & & & \\
\hline & Tritium/P.S. & 309.5 & & & N/A & & & & & \\
\hline & & & N/A & Shoe & $312.5-313.1$ & & 241 & ND & & \\
\hline & & & $\mathrm{B} 1 \mathrm{~K} 9 \mathrm{~N} 3$ & A & $312.0-312.5$ & & 95 & 100 & है & \\
\hline & Split Spoon & $310.5-313.1$ & B1K9N4 & B & $311.5-312.0$ & 416 & 35 & 100 & 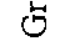 & \\
\hline 11-Aug & & & B1K9N5 & $\mathrm{C}$ & $311.0-311.5$ & & 24 & 100 & 客 & \\
\hline & & & B1K9N6 & $\mathrm{D}$ & $310.5-311.0$ & & 11 & 90 & 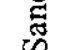 & \\
\hline & Drive Barrel & $310.5-313.0$ & B1K9N7 & & N/A & & & & & \\
\hline & Tritium & 313.0 & & & N/A & & & & & \\
\hline
\end{tabular}


WMP-32119, Rev. 0

Table 2-4. Sediment Sampling Summary for Entry Borehole C4997 (6 pages)

\begin{tabular}{|c|c|c|c|c|c|c|c|c|c|c|}
\hline $\begin{array}{c}\text { Date } \\
(2006)\end{array}$ & $\begin{array}{c}\text { Sample } \\
\text { Type }\end{array}$ & $\begin{array}{l}\text { Depth Intv. } \\
\text { (ft bgs) }\end{array}$ & $\begin{array}{c}\text { HEIS } \\
\text { Number }\end{array}$ & Liner & $\begin{array}{l}\text { Liner } \\
\text { Depth }\end{array}$ & \multicolumn{2}{|c|}{$\begin{array}{c}\text { Blow } \\
\text { Counts }\end{array}$} & $\begin{array}{c}\% \\
\text { Rec. }\end{array}$ & \multicolumn{2}{|c|}{ Strat. } \\
\hline \multirow{8}{*}{ 15-Aug } & Tritium/P.S. & 335.0 & \multicolumn{6}{|c|}{ N/A } & \multirow{11}{*}{ 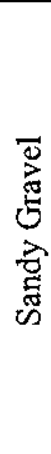 } & \multirow{24}{*}{ 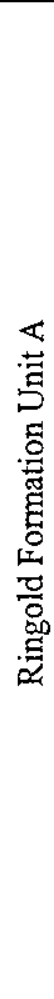 } \\
\hline & \multirow{5}{*}{$\begin{array}{l}\text { Split Spoon } \\
\text { (FH VOA } \\
\text { Samples) }\end{array}$} & \multirow{5}{*}{$336.8-339.2$} & N/A & Shoe & $338.8-339.2$ & \multirow{5}{*}{680} & 289 & \multirow{5}{*}{100} & & \\
\hline & & & \multirow{4}{*}{$\begin{array}{l}\text { B1K9D0 } \\
\text { B1K9D1 } \\
\text { B1K9D2 }\end{array}$} & $\mathrm{A}$ & $338.3-338.8$ & & 120 & & & \\
\hline & & & & $\mathrm{B}$ & $337.8-338.3$ & & 145 & & & \\
\hline & & & & $\mathrm{C}$ & $337.3-337.8$ & & 56 & & & \\
\hline & & & & $\mathrm{D}$ & $336.8-337.3$ & & 30 & & & \\
\hline & Drive Barrel & $310.5-313.0$ & B1K9F7 & & N/A & & & & & \\
\hline & Tritium & 313.0 & \multicolumn{6}{|c|}{ N/A } & & \\
\hline \multirow{3}{*}{ 16-Aug } & Tritium/P.S. & 351.0 & \multicolumn{6}{|c|}{$\mathrm{N} / \mathrm{A}$} & & \\
\hline & Drive Barrel & $351.0-352.5$ & B1K9F8 & \multicolumn{4}{|c|}{ N/A } & 30 & & \\
\hline & Tritium & 353.0 & \multicolumn{6}{|c|}{ N/A } & & \\
\hline \multirow{9}{*}{ 16-Aug } & Tritium & 366.0 & \multicolumn{6}{|c|}{ N/A } & \multirow{13}{*}{ 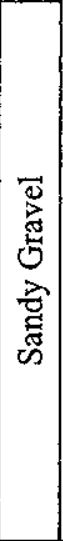 } & \\
\hline & P.S. & $365.6-367.6$ & \multicolumn{6}{|c|}{ N/A } & & \\
\hline & \multirow{5}{*}{$\begin{array}{c}\text { Split Spoon } \\
\text { (FH VOA } \\
\text { Samples) }\end{array}$} & \multirow{5}{*}{$365.6-367.1$} & $\mathrm{~N} / \mathrm{A}$ & Shoe & $366.6-367.1$ & \multirow{3}{*}{145} & 71 & $\mathrm{ND}$ & & \\
\hline & & & $\mathrm{B} 1 \mathrm{KCO5}$ & $\mathrm{A}$ & $366.1-366.6$ & & 35 & 100 & & \\
\hline & & & $\mathrm{B} 1 \mathrm{KC} 06$ & $\mathrm{~B}$ & $365.6-366.1$ & & 39 & 100 & & \\
\hline & & & $\mathrm{B} 1 \mathrm{KC} 07$ & & NR & & & $5 0 \longdiv { 0 }$ & & \\
\hline & & & $\mathrm{B} 1 \mathrm{KC} 08$ & & NR & & & 0 & & \\
\hline & Drive Barrel & $365.6-367.6$ & B1KB68 & & N/A & & & 50 & & \\
\hline & Tritium & 313.0 & \multicolumn{6}{|c|}{ N/A } & & \\
\hline \multirow{4}{*}{ 17-Aug } & Tritium & 380.0 & \multicolumn{6}{|c|}{ N/A } & & \\
\hline & P.S. & $380.0-382.0$ & \multicolumn{6}{|c|}{$\mathrm{N} / \mathrm{A}$} & & \\
\hline & Drive Barrel & $380.0-382.0$ & B1K8F9 & \multicolumn{5}{|c|}{$\mathrm{N} / \mathrm{A}$} & & \\
\hline & Tritium & 385.0 & \multicolumn{6}{|c|}{ N/A } & & \\
\hline \multicolumn{11}{|c|}{$\begin{array}{l}\text { Notes: } \\
\text { ft bgs = below ground s } \\
\mathrm{ft}=\text { feet } \\
\text { Jul = July } \\
\text { Aug = August } \\
\mathrm{N} / \mathrm{A}=\text { not applicable } \\
\mathrm{ND}=\text { no data } \\
\text { Strat. = Stratigraphy }\end{array}$} \\
\hline
\end{tabular}

Table 2-5. Water Sampling Summary for Entry Borehole C4997

\begin{tabular}{|c|c|c|c|c|c|}
\hline $\begin{array}{c}\text { Date } \\
\text { (2006) }\end{array}$ & $\begin{array}{c}\text { Sample } \\
\text { Type }\end{array}$ & $\begin{array}{c}\text { Sampling } \\
\text { Method }\end{array}$ & $\begin{array}{c}\text { Depth Intv. (ft } \\
\text { bgs) }\end{array}$ & $\begin{array}{c}\text { HEIS } \\
\text { Numbers }\end{array}$ & $\begin{array}{c}\text { Duplicate Set HEIS } \\
\text { Numbers }\end{array}$ \\
\hline 9-Aug & Water & Kabis & $279.5-280.4$ & $\begin{array}{c}\text { B1K996 } \\
\text { B1K997 }\end{array}$ & N/A \\
\hline 10-Aug & Water & Kabis & 303.0 & $\begin{array}{l}\text { B1K9B0 } \\
\text { B1K9B1 }\end{array}$ & $\begin{array}{l}\text { B1K9B2 } \\
\text { B1K9B3 }\end{array}$ \\
\hline 15-Aug & Water & Kabis & $334.5-339.0$ & $\begin{array}{l}\text { B1K9B4 } \\
\text { B1K9B5 }\end{array}$ & N/A \\
\hline 16-Aug & Water & Kabis & $364.5-367.6$ & $\begin{array}{l}\text { B1KB73 } \\
\text { B1KB74 }\end{array}$ & N/A \\
\hline
\end{tabular}

Notes:

$\mathrm{ft}$ bgs $=$ feet below ground surface 
WMP-32119, Rev. 0, RE-ISSUE

\begin{tabular}{|c|c|c|c|c|c|}
\hline $\begin{array}{c}\text { Date } \\
(2006)\end{array}$ & $\begin{array}{c}\text { Sample } \\
\text { Type }\end{array}$ & $\begin{array}{c}\text { Sampling } \\
\text { Method }\end{array}$ & $\begin{array}{c}\text { Depth Intv. (ft } \\
\text { bgs) }\end{array}$ & $\begin{array}{c}\text { HEIS } \\
\text { Numbers }\end{array}$ & $\begin{array}{c}\text { Duplicate Set HEIS } \\
\text { Numbers }\end{array}$ \\
\hline
\end{tabular}

Aug $=$ August

\subsubsection{Construction Summary}

Entry Borehole construction for borehole $\mathrm{C} 4997$ was designed to enable deep mud-rotary drilling to advance the borehole to approximately $1300 \mathrm{ft}$ to $1500 \mathrm{ft}$ bgs. After a total depth of $401.0 \mathrm{ft}$ bgs ( $18 \mathrm{ft}$ into basalt) was obtained, a grout seal was installed from about $330 \mathrm{ft}$ to $401.0 \mathrm{ft} \mathrm{bgs,}$ consisting of Portland Type I/II cement and 5\% bentonite powder by volume per WAC 173-160. After the cement grout was in place the $95 / 8$-inch temporary casing was backpulled $\sim 5 \mathrm{ft}$ to allow the cement to make contact with the formation and then driven back to $383 \mathrm{ft} \mathrm{bgs}$. Borehole plumbness testing was performed at approximately 50-ft intervals during borehole advancement using a $103 / 4$-inch OD, 20-ft long tool within the 13 3/8-inch OD casing and a 6 $5 / 8$-inch OD, 20-ft long tool within the $95 / 8$-inch OD casing. Additional plumbness testing was performed at approximately $50-\mathrm{ft}$ intervals using a gyro survey tool. A separate report will provide specific details of these gyro surveys. Construction and completion of this entry borehole was carried out from August 18, 2006 to August 19,2006. Well construction summary data are provided in Table 2-6.

Table 2-6. Construction Summary for Entry Borehole C4997

\begin{tabular}{|c|c|c|c|c|c|c|}
\hline $\begin{array}{l}\text { Borehole } \\
\text { ID }\end{array}$ & $\begin{array}{l}\text { Borehole } \\
\text { Location }\end{array}$ & $\begin{array}{l}\text { Total Depth } \\
\text { (ft bgs) }\end{array}$ & $\begin{array}{l}\text { Water Level } \\
\text { (ft bgs) }\end{array}$ & $\begin{array}{l}133 / 8 \text { in. OD } \\
\text { casing depth }\end{array}$ & $\begin{array}{l}95 / 8 \text { in. OD } \\
\text { casing depth }\end{array}$ & $\begin{array}{c}\text { Grout Seal } \\
\text { Interval }\end{array}$ \\
\hline C4997 & WTP Site & 401.0 & 277.5 & 216.5 & 383.3 & $330-401$ \\
\hline \multicolumn{7}{|c|}{$\begin{array}{l}\text { Notes: } \\
\mathrm{ft} \text { bgs }=\text { feet below ground surface. } \\
\text { in. }=\text { inch } \\
\mathrm{OD}=\text { outside diameter. }\end{array}$} \\
\hline
\end{tabular}

\subsubsection{Entry Borehole C4993}

This section summarizes activities related to the construction of entry borehole $\mathrm{C} 4993$

\subsubsection{Drilling Summary}

Drilling of entry borehole C4993 began on August 21, 2006 using a cable tool drill rig, driving single wall carbon steel temporary casing with a $133 / 8$-inch OD and $123 / 8$-inch ID to a depth of $211.8 \mathrm{ft}$ below ground surface (bgs). Below $211.8 \mathrm{ft}$ bgs the casing was downsized to single wall carbon steel temporary casing with a $95 / 8$-inch OD and $85 / 8$-inch ID that was driven to a depth of $363.7 \mathrm{ft}$ bgs. The borehole was advanced using both core barrel and hard tool drilling methods to a total depth of $383.5 \mathrm{ft}$ bgs on September 4,2006. The water table was initially encountered at approximately 258.2 feet bgs on August 29, 2006. 


\subsubsection{Sample Summary}

Field sampling data are presented in Table 2.7. Four split spoon samples and four corresponding drive barrel samples were collected at discrete intervals and will be used in a laboratory testing program to obtain site-specific soil property data. Soil testing procedures are detailed in ASTM D4015-92 (Standard Test Methods for Modulus and Damping of Soils by the Resonant-Column Method) and GR06-4 (Test Procedures and Calibration Documentation Associated with the RCTS and URC Tests at the University of Texas at Austin). Below groundwater, soil samples (1-pint glass jars) were collected directly above and below each split spoon interval and will be used in laboratory testing for tritium analysis. Additional soil samples (1-pint glass jars) were collected at each split spoon interval and will be used for particle size analysis. Archive samples (1-pint glass jars) were collected for FH and PNNL, at five-ft intervals, but were not analyzed in the field. In addition to the archive samples, lithologic changes were recorded and collected in plastic chip trays for future characterization use by both FH and PNNL. 
WMP-32119, Rev. 0

Table 2-7. Sediment Sampling Summary for Entry Borehole C4993

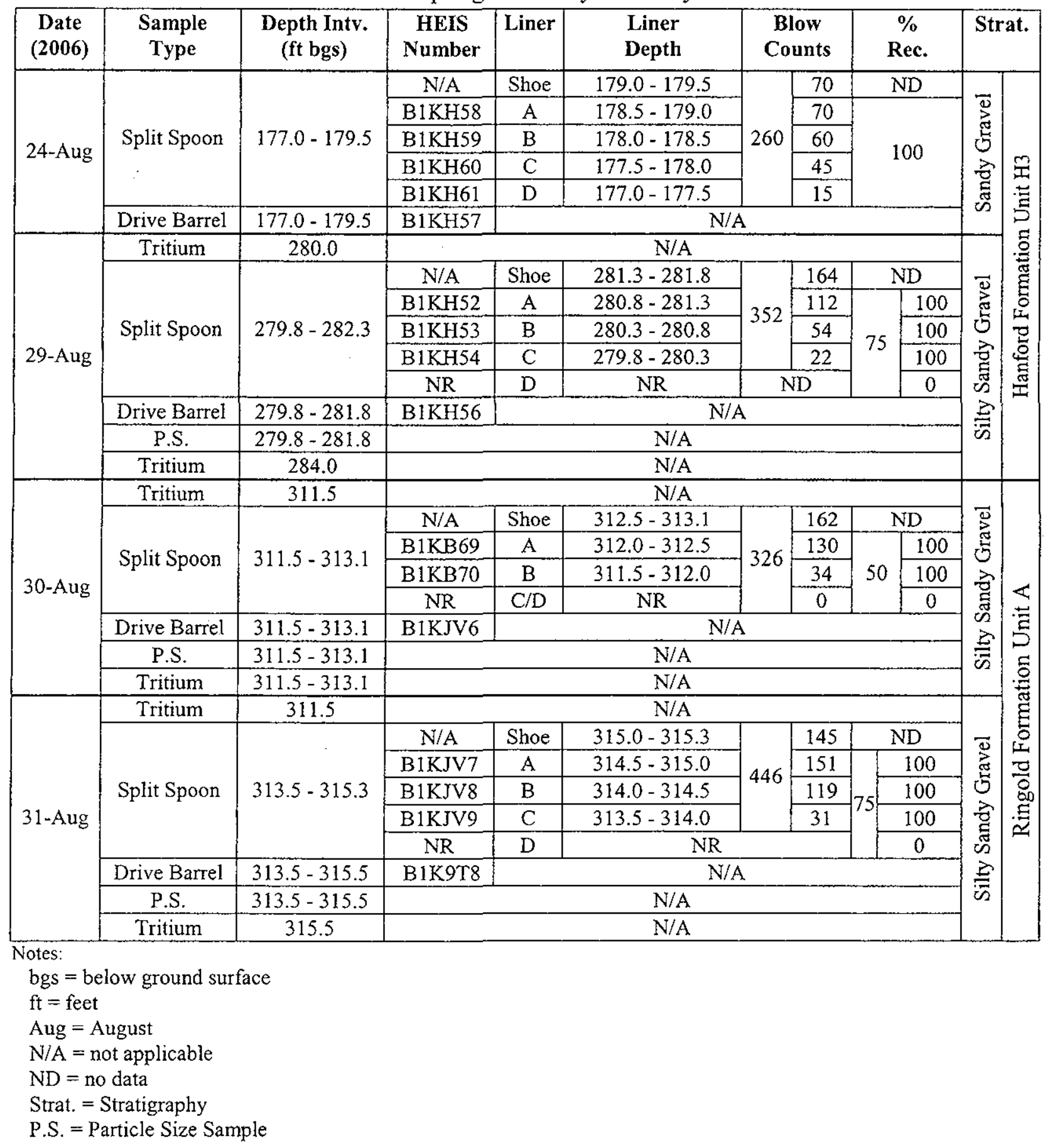

\subsubsection{Construction Summary}

Entry Borehole construction for borehole $\mathrm{C} 4993$ was designed to enable deep mud-rotary drilling to advance the borehole to approximately $1300 \mathrm{ft}$ to $1500 \mathrm{ft}$ bgs. After a total depth 
of $383.5 \mathrm{ft}$ bgs ( $25.5 \mathrm{ft}$ into basalt) was obtained, a grout seal was installed from about 354.7 $\mathrm{ft}$ to $383.5 \mathrm{ft}$ bgs, consisting of Portland Type I/II cement and $5 \%$ bentonite powder by volume per WAC 173-160. After the cement grout was in place the $95 / 8$-inch temporary casing was backpulled $5 \mathrm{ft}$ to allow the cement to make contact with the formation and then driven back to $383.5 \mathrm{ft}$ bgs. Borehole plumbness testing was performed at approximately 50$\mathrm{ft}$ intervals during borehole advancement using a $103 / 4$-inch $\mathrm{OD}, 20$ - $\mathrm{ft}$ long tool within the 13 3/8-inch OD casing and a $65 / 8$-inch OD, 20-ft long tool within the $95 / 8$-inch OD casing. Additional plumbness testing was performed at approximately $50-\mathrm{ft}$ intervals using a gyro survey tool. A separate report will provide specific details of these gyro surveys.

Construction and completion of this entry borehole was carried out on September 5, 2006. Well construction summary data are provided in Table 2-8.

Table 2-8. Construction Summary for Entry Borehole C4993

\begin{tabular}{|c|c|c|c|c|c|c|}
\hline $\begin{array}{l}\text { Borehole } \\
\text { ID }\end{array}$ & $\begin{array}{l}\text { Borehole } \\
\text { Location }\end{array}$ & $\begin{array}{l}\text { Total Depth } \\
\text { (ft bgs) }\end{array}$ & $\begin{array}{l}\text { Water Level } \\
\text { (ft bgs) }\end{array}$ & $\begin{array}{l}133 / 8 \text { in. OD } \\
\text { casing depth }\end{array}$ & $\begin{array}{l}95 / 8 \text { in. OD } \\
\text { casing depth }\end{array}$ & $\begin{array}{l}\text { Grout Seal } \\
\text { Interval }\end{array}$ \\
\hline $\mathrm{C} 4993$ & WTP Site & 383.5 & 258.2 & 211.8 & 363.7 & $354.7-383.5$ \\
\hline \multicolumn{7}{|c|}{$\begin{array}{l}\text { Notes: } \\
\mathrm{ft} \text { bgs = feet below ground surface. } \\
\text { in. }=\text { inch } \\
\mathrm{OD}=\text { outside diameter. }\end{array}$} \\
\hline
\end{tabular}


WMP-32119, Rev. 0

This page intentionally left blank. 


\subsection{WASTE MANAGEMENT}

The Unit Managers for Comprehensive Environmental Response, Compensation, and Liability Act (CERCLA) Operable Units at the Hanford Site issued a decision to include these test boreholes and corehole in the 200-PO-1 Operable Unit. Under this agreement, waste from the drilling of these holes was disposed of according to DOE/RL-2004-18, Waste Control Plan for the 200-PO-1 Operable Unit (February 2004). Waste was designated in accordance with WAC 173-303 and RCW 70.105 using a combination of process knowledge, historical analytical data, and sample analysis. All details concerning handling of waste are addressed by Gardner et al., (2006) and the Waste Control Plan. Final waste management instructions in the field were provided and controlled by the drilling contractor (Energy Solutions), with additional details provided below.

\subsection{WTP SITE (200-PO-1 OU)}

Cutting spoils for the WTP site were handled as described in the following sections.

\subsubsection{Vadose Zone Cuttings}

Vadose zone cuttings from the WTP site were designated low risk from chemical or radiological contamination. Vadose zone cuttings from boreholes C4998, C4996 and C4993 were collected in stockpiles near the point of generation and then released back into the ground based on field surveys by radiological control technicians (RCT). All drill cuttings generated from ground surface to TD from entry borehole $\mathrm{C} 4997$ were containerized in 55-gallon drums lined with a 10 mil plastic liner. Drums were stored on site for final disposition. Drill cuttings were surveyed in accordance with Hanford Site Solid Waste Acceptance Criteria (HNF-EP-0063).

All wastes generated from drilling and sampling operations were handled as CERCLA waste and were managed in accordance with the DOE/RL-2004-18, Waste Control Plan for the 200-PO-1 Operable Unit (February 2004).

\subsubsection{Saturated Zone Cuttings}

All drill cuttings below the highest recorded water table (approximately $220 \mathrm{ft}$ bgs in borehole C4998, $220 \mathrm{ft}$ bgs in borehole C4996 and $215 \mathrm{ft}$ bgs in borehole C4993) were containerized in 55 -gallon drums lined with a 10-mil plastic liner. Drums were stored on site for final disposition. 
WMP-32119, Rev. 0

This page intentionally left blank. 


\section{WMP-32119, Rev. 0}

\subsection{GEOPHYSICAL SURVEY}

Borehole geophysical surveys were performed in entry borehole C4998 on June 22, 2006 and July 10, 2006. Spectral Gamma Logging System (SGLS) and bulk density surveys were carried out from ground surface ( 0 feet) to 392 feet bgs. Survey results are plotted in figure 4-1. A separate report will provide specific details of these geophysical surveys. 


\section{WMP-32119, Rev. 0}

Figure 4-1: Geophysical Data for Seismic Entry Borehole C4998

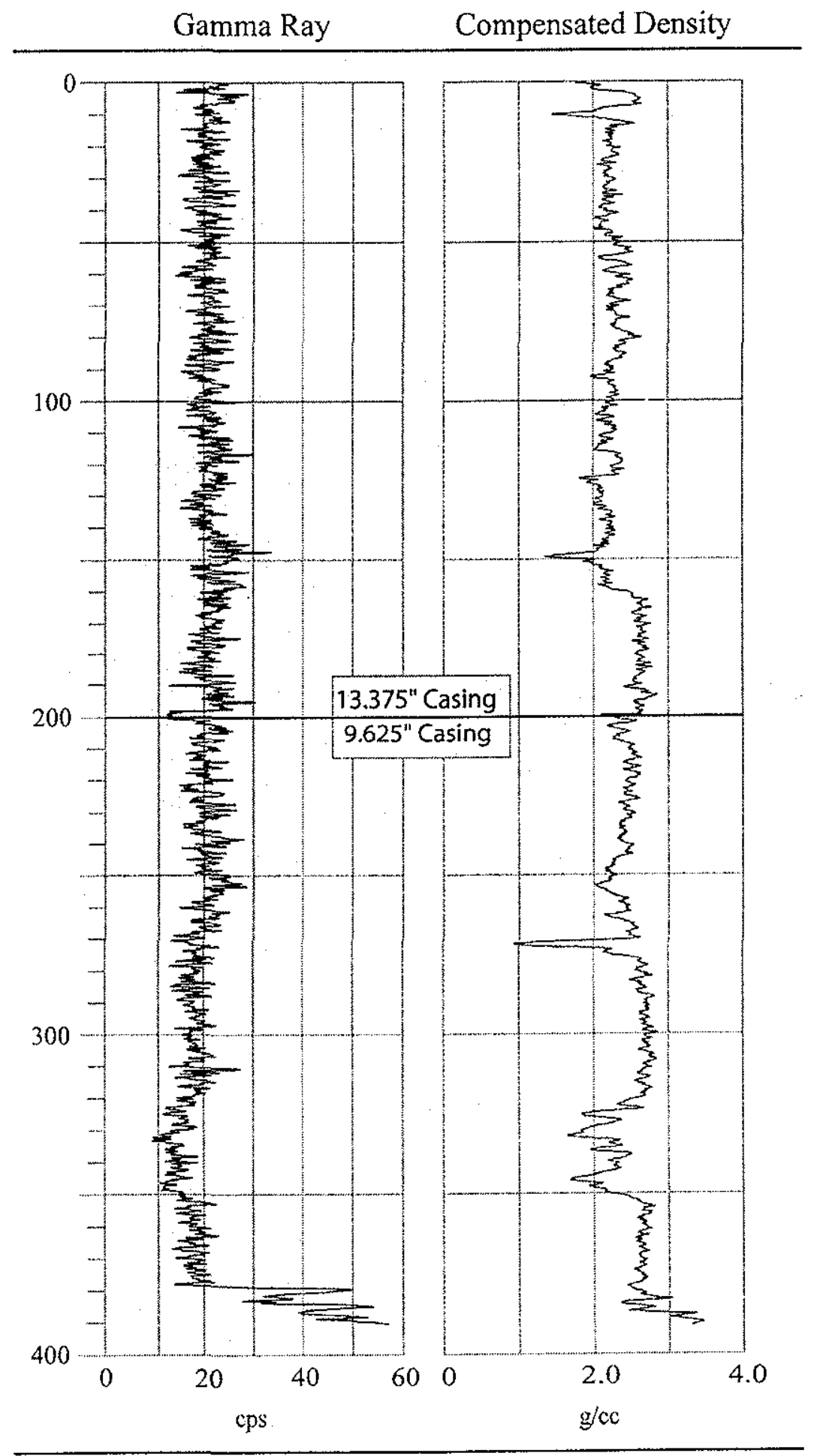


WMP-32119, Rev. 0

\subsection{CIVIL SURVEY}

The civil surveys of all four boreholes had not been completed at the time of report preparation. When available, the civil survey data will be entered into the Hanford Well Information System (HWIS) database. 
WMP-32119, Rev. 0

This page intentionally left blank. 
WMP-32119, Rev. 0

\subsection{SUBSURFACE DESCRIP'TION}

\subsection{WTP SITE}

This section provides the generalized stratigraphy of the WTP site, as well as summaries of field observations

\subsubsection{Geology/hydrogeology}

Generalized stratigraphy beneath the WTP Site includes sediment backfill that primarily consist of sand, generally less than $5 \mathrm{~m}$ in thickness. The backfill sediments overlie unconsolidated sediments of the Hanford formation. The Hanford formation extends for approximately $250 \pm$ $20 \mathrm{ft}$ and consists primarily of fine- course-grained sand, rare silt to clayey silt, and pebble- to boulder-gravel fluvial deposits. At the WTP site these deposits are divided into two groups, sand-dominated (H2 Unit) and gravel-dominated (H3 Unit). The sand-dominated group consists of fine- course-grained sand with variable silt content. The sand typically contains a high basalt content that generally decreases within layers of fine- to very-fine-grained sand. The sand commonly contains small pebbles and intermittent, felsic-dominated silty layers less than $1.0 \mathrm{~m}$ thick. The gravel-dominated group consists of coarse-grained sand and granule-to-boulder gravel. The gravel is typically clast-supported and contains a high basalt content. In general, the basalt content decreases with depth, forming a gradual transition to relatively older, more heterolithic gravel deposits. These older deposits, herein referred to as "reworked Ringold Formation", commonly exhibit a slightly higher degree of weathering and were likely derived from scouring and reworking of the underlying Ringold Formation. The reworked Ringold Formation deposits overlie consolidated sandy gravel from Unit A of the Ringold Formation. Sediment of Ringold Formation Unit A beneath the WTP site typically consists of sub- to wellrounded heterolithic pebbles and small cobbles with a felsic-dominated medium-grained sand matrix that is commonly distinguished by its striking light olive brown color. The Ringold Formation disconformably overlies basalt of the Elephant Mountain Member of the Columbia River Basalt Group. A summary of stratigraphic units encountered at the WTP entry borehole sites is presented in Table 2-9. 
WMP-32119, Rev. 0

Table 2-9. Suprabasalt Sedimentary Units Encountered at the WTP Entry Borehole Sites

\begin{tabular}{|c|c|c|c|c|c|c|c|c|}
\hline $\begin{array}{c}\text { Borehole } \\
\text { ID }\end{array}$ & \multicolumn{2}{|c|}{$\begin{array}{l}\text { C4998 } \\
\text { Center }\end{array}$} & \multicolumn{2}{|c|}{$\begin{array}{c}\text { C4996 } \\
\text { Northwest }\end{array}$} & \multicolumn{2}{|c|}{$\begin{array}{l}\text { C4997 } \\
\text { Center } \\
\end{array}$} & \multicolumn{2}{|c|}{$\begin{array}{c}\text { C4993 } \\
\text { Southwest }\end{array}$} \\
\hline $\begin{array}{c}\text { Surface } \\
\text { Elevation }\end{array}$ & \multicolumn{2}{|c|}{ NS } & \multicolumn{2}{|c|}{ NS } & \multicolumn{2}{|c|}{ NS } & \multicolumn{2}{|c|}{ NS } \\
\hline Unit & $\begin{array}{l}\text { Thickness } \\
\text { (feet) }\end{array}$ & $\begin{array}{l}\text { Drilled } \\
\text { Depth }^{2}\end{array}$ & $\begin{array}{c}\text { Thickness } \\
\text { (feet) }\end{array}$ & $\begin{array}{l}\text { Drilled } \\
\text { Depth }^{a}\end{array}$ & $\begin{array}{l}\text { Thickness } \\
\text { (feet) }\end{array}$ & $\begin{array}{l}\text { Drilled } \\
\text { Depth }^{a}\end{array}$ & $\begin{array}{l}\text { Thickness } \\
\text { (feet) }\end{array}$ & $\begin{array}{l}\text { Drilled } \\
\text { Depth }^{\mathrm{a}}\end{array}$ \\
\hline $\begin{array}{l}\text { Suprabasalt } \\
\text { Sediments }\end{array}$ & 382 & 0 & 349 & 0 & 383 & 0 & 358 & 0 \\
\hline Backfill & 9 & 0 & 0.5 & 0 & 16.8 & 0 & 0.3 & 0 \\
\hline $\begin{array}{c}\text { Hanford } \\
\text { fm. Unit } \\
\mathrm{H} 2\end{array}$ & 151.5 & 9 & 165 & 0.5 & 149.2 & 16.8 & 159.7 & 0.3 \\
\hline $\begin{array}{c}\text { Hanford } \\
\text { fm. Unit } \\
\text { H3 }\end{array}$ & 90.5 & 159.5 & 89.5 & 165.5 & 89.5 & 166 & 70 & 166 \\
\hline $\begin{array}{l}\text { Reworked } \\
\text { Ringold } \\
\text { Fm. }\end{array}$ & 72 & 250 & 49 & 255 & 63.5 & 255.5 & 64 & 236 \\
\hline $\begin{array}{c}\text { Ringold } \\
\text { Fm. Unit A }\end{array}$ & 60 & 322 & 45 & 304 & 64 & 319 & 58 & 300 \\
\hline $\begin{array}{l}\text { Notes: } \\
\text { a Top of } \\
\text { NS = No }\end{array}$ & $\begin{array}{l}\text { it in feet } \\
\text { Irvey ava }\end{array}$ & 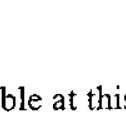 & & & & & & \\
\hline
\end{tabular}

\subsubsection{Entry Borehole C4998}

At this location, in-situ sediments are overlain by a backfill layer of sand intermixed with aeolian sand and silt, which extend from ground surface to a depth of about $9.0 \mathrm{ft}$ bgs. Sediment below the sand fill, from 9.0-159.5 feet bgs consists of Unit $\mathrm{H} 2$ sand-dominated sediment of the Hanford formation. The interval from 159.5-250.0 ft bgs contains Unit $\mathrm{H} 3$ gravel-dominated sediment of the Hanford formation. The Hanford formation overlies reworked sediments of the Ringold Formation, which extend from 250-322.0 ft bgs. Sediments of the Ringold Formation Unit A were encountered between $322.0 \mathrm{ft}$ bgs to $382 \mathrm{ft}$ bgs. Basalt of the Elephant Mountain Member of the Columbia River Basalt Group was encountered from $382 \mathrm{ft}$ bgs to a total entry borehole depth of $401.5 \mathrm{ft}$ bgs. Lithologic descriptions and geologic borehole logs for this borehole were prepared in accordance with FH procedure CP-GPP-EE-01-7.0, Rev. 1, "Geologic Logging," and are included in Appendix A.

\subsubsection{Entry Borehole C4996}

At this location, in-situ sediments are overlain by a backfill layer of crushed gravel intermixed with aeolian sand and silt, which extend from ground surface to a depth of about $0.5 \mathrm{ft}$ bgs. Sediment below the gravel fill, from $0.5-165.5$ feet bgs consists of Unit $\mathrm{H} 2$ sand-dominated sediment of the Hanford formation. The interval from 165.5-255.0 ft bgs contains Unit H3 gravel-dominated sediment of the Hanford formation. The gravel-dominated sequence at this 
site, however, contains several intermittent layers of sand-dominated sediment. The Hanford formation overlies reworked sediments of the Ringold Formation, which extend from 255.0$304.0 \mathrm{ft}$ bgs. Sediments of the Ringold Formation Unit A were encountered between $304.0 \mathrm{ft}$ bgs to $349 \mathrm{ft}$ bgs. Basalt of the Elephant Mountain Member of the Columbia River Basalt Group was encountered from $349.0 \mathrm{ft}$ bgs to a total entry borehole depth of $369.0 \mathrm{ft}$ bgs. Lithologic descriptions and geologic borehole logs for this well were prepared in accordance with FH procedure CP-GPP-EE-01-7.0, Rev. 1, "Geologic Logging," and are included in Appendix B.

\subsubsection{Entry Borehole C4997}

At this location, in-situ sediments are overlain by a backfill layer of sand intermixed with aeolian sand and silt, which extend from ground surface to a depth of about $16.8 \mathrm{ft}$ bgs. Sediment below the sand fill, from 16.8-166.0 feet bgs consists of Unit H2 sand-dominated sediment of the Hanford formation. The interval from 166.0-255.5 ft bgs contains Unit H3 gravel-dominated sediment of the Hanford formation. The Hanford formation overlies reworked sediments of the Ringold Formation, which extend from 255.5-319.0 ft bgs. Sediments of the Ringold Formation Unit A were encountered between $319.0 \mathrm{ft}$ bgs to $383.0 \mathrm{ft}$ bgs. Basalt of the Elephant Mountain Member of the Columbia River Basalt Group was encountered from $383.0 \mathrm{ft}$ bgs to a total entry borehole depth of $401.0 \mathrm{ft}$ bgs. Lithologic descriptions and geologic borehole logs for this well were prepared in accordance with FH procedure CP-GPP-EE-01-7.0, Rev. 1, "Geologic Logging," and are included in Appendix C.

\subsubsection{Entry Borehole C4993}

At this location, in-situ sediments are overlain by a backfill layer of crushed gravel intermixed with aeolian sand and silt, which extend from ground surface to a depth of about $0.3 \mathrm{ft}$ bgs. Sediment below the backfill, from $0.3-166.0$ feet bgs consists of Unit $\mathrm{H} 2$ sand-dominated sediment of the Hanford formation. The interval from 166.0-236.0 ft bgs contains Unit $\mathrm{H} 3$, gravel-dominated sediment of the Hanford formation. The Hanford formation overlies reworked sediments of the Ringold Formation, which extend from $236.0-300 \mathrm{ft}$ bgs. Sediments of the Ringold Formation Unit A were encountered between 300.0-358.0 ft bgs. Basalt of the Elephant Mountain Member of the Columbia River Basalt Group was encountered from $358.0 \mathrm{ft}$ bgs to a total entry borehole depth of $383.5 \mathrm{ft}$ bgs. Lithologic descriptions and geologic borehole logs for this well were prepared in accordance with FH procedure CP-GPP-EE-01-7.0, Rev. 1, "Geologic Logging," and are included in Appendix D. 
WMP-32119, Rev. 0

Figure 6-1: Subsurface Geology and Geophysical Data for Seismic Entry Borehole C4998

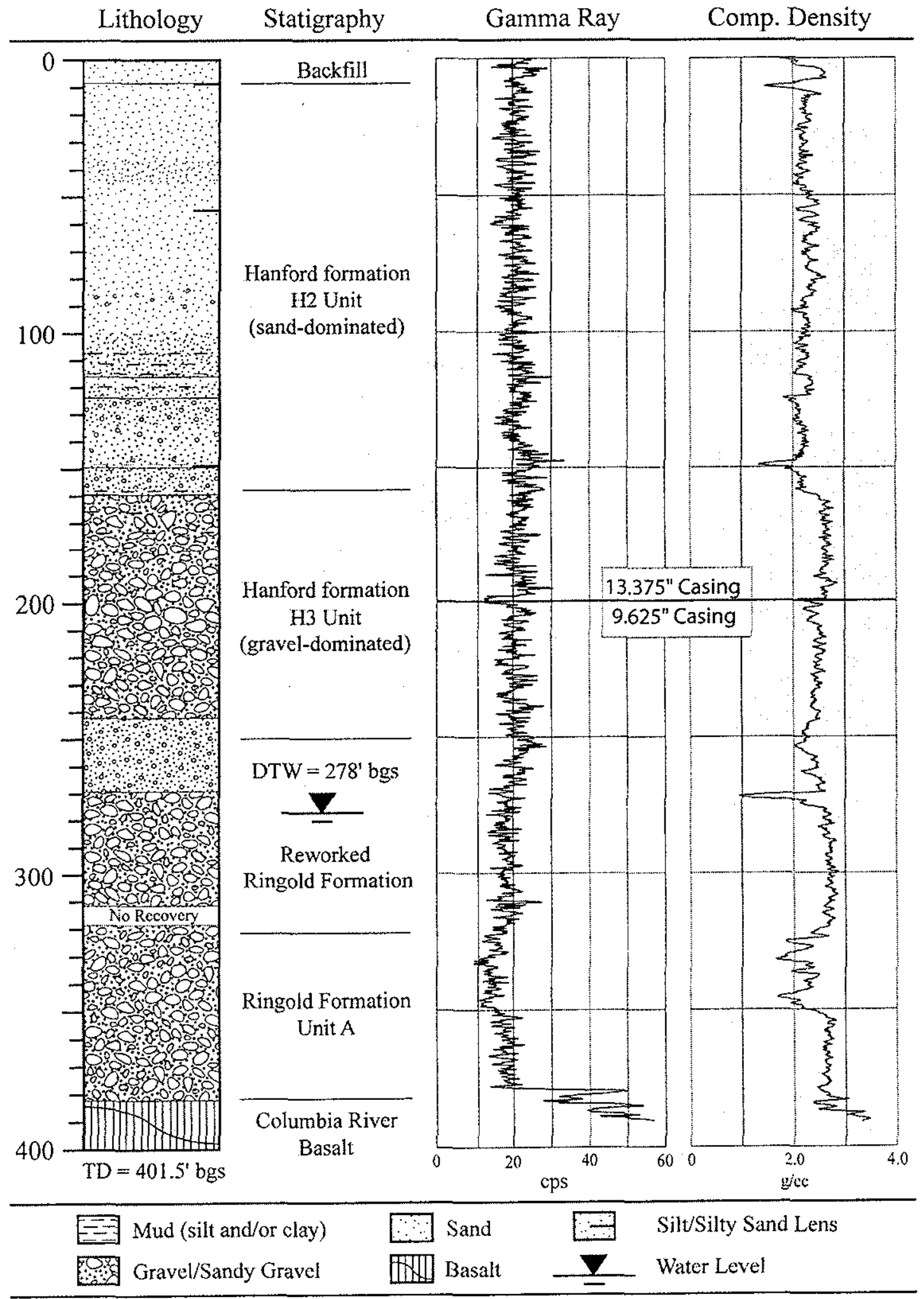


WMP-32119, Rev. 0

Figure 6-2: Subsurface Geology and Soil Sampling Intervals for Seismic Entry Borehole C4996

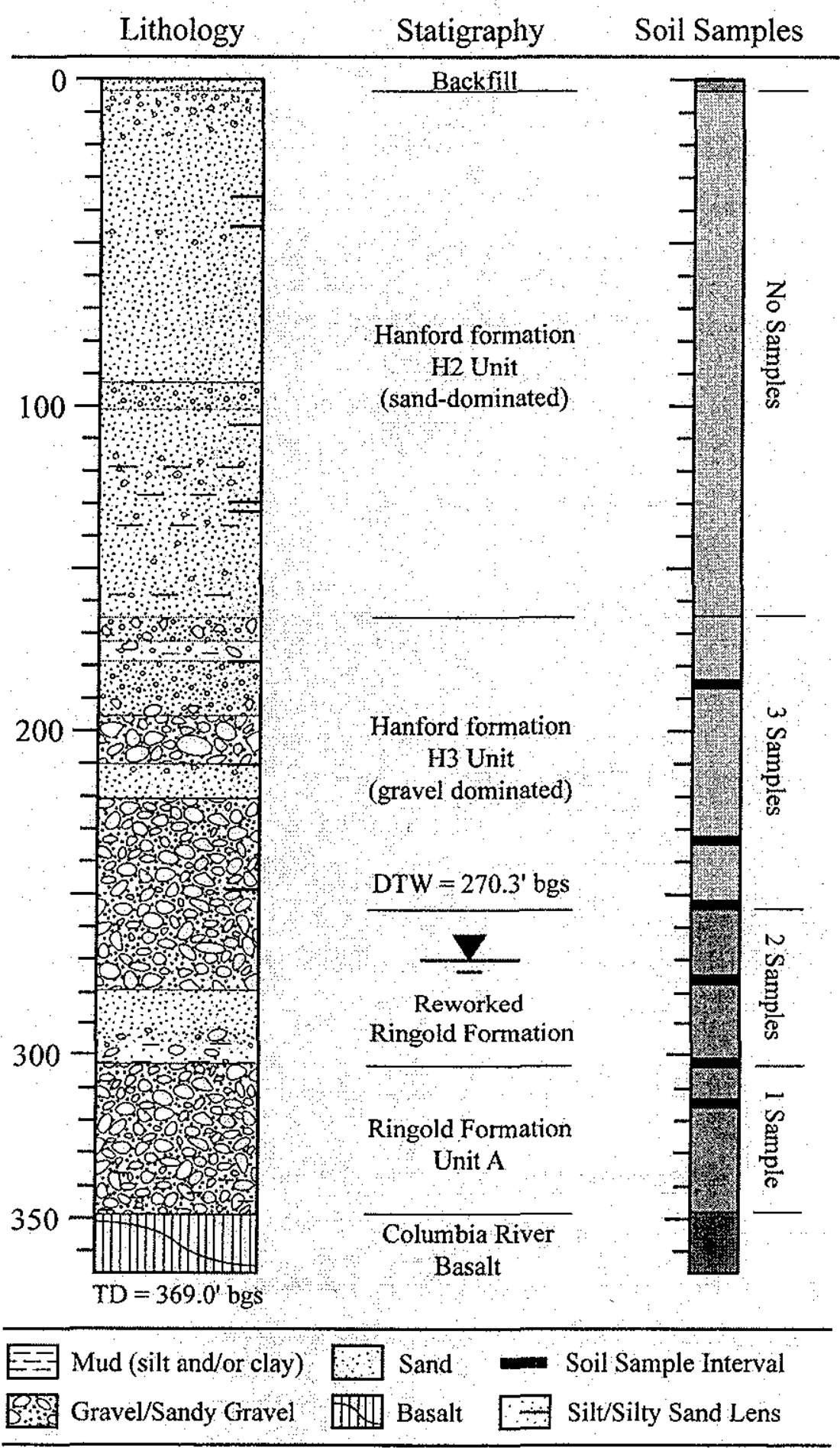


WMP-32119, Rev. 0

Figure 6-3: Subsurface Geology and Soil Sampling Intervals for Seismic Entry Borehole C4997

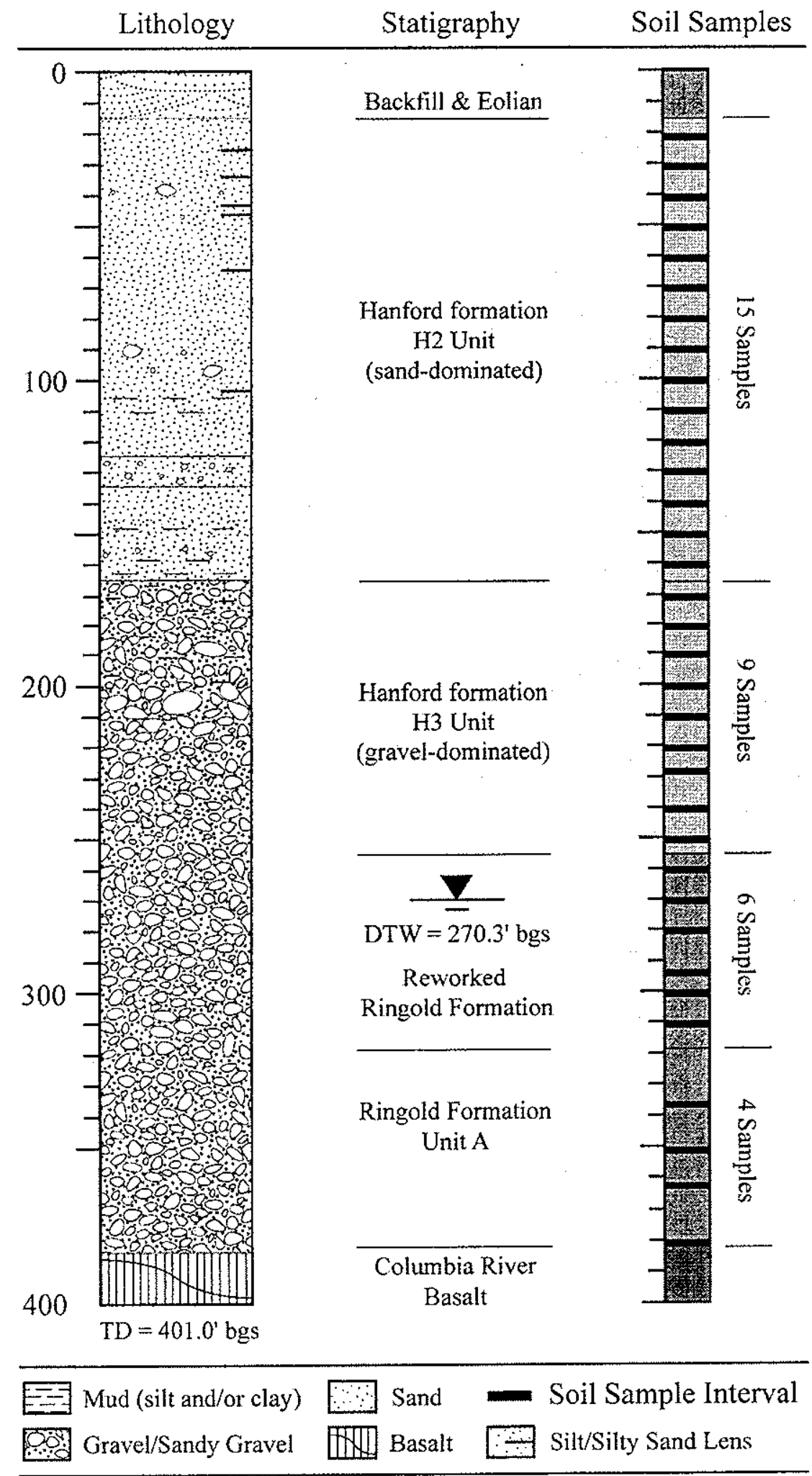

Figure 6-4: Subsurface Geology and Soil Sampling Intervals for Seismic Entry Borehole C4993 
WMP-32119, Rev. 0

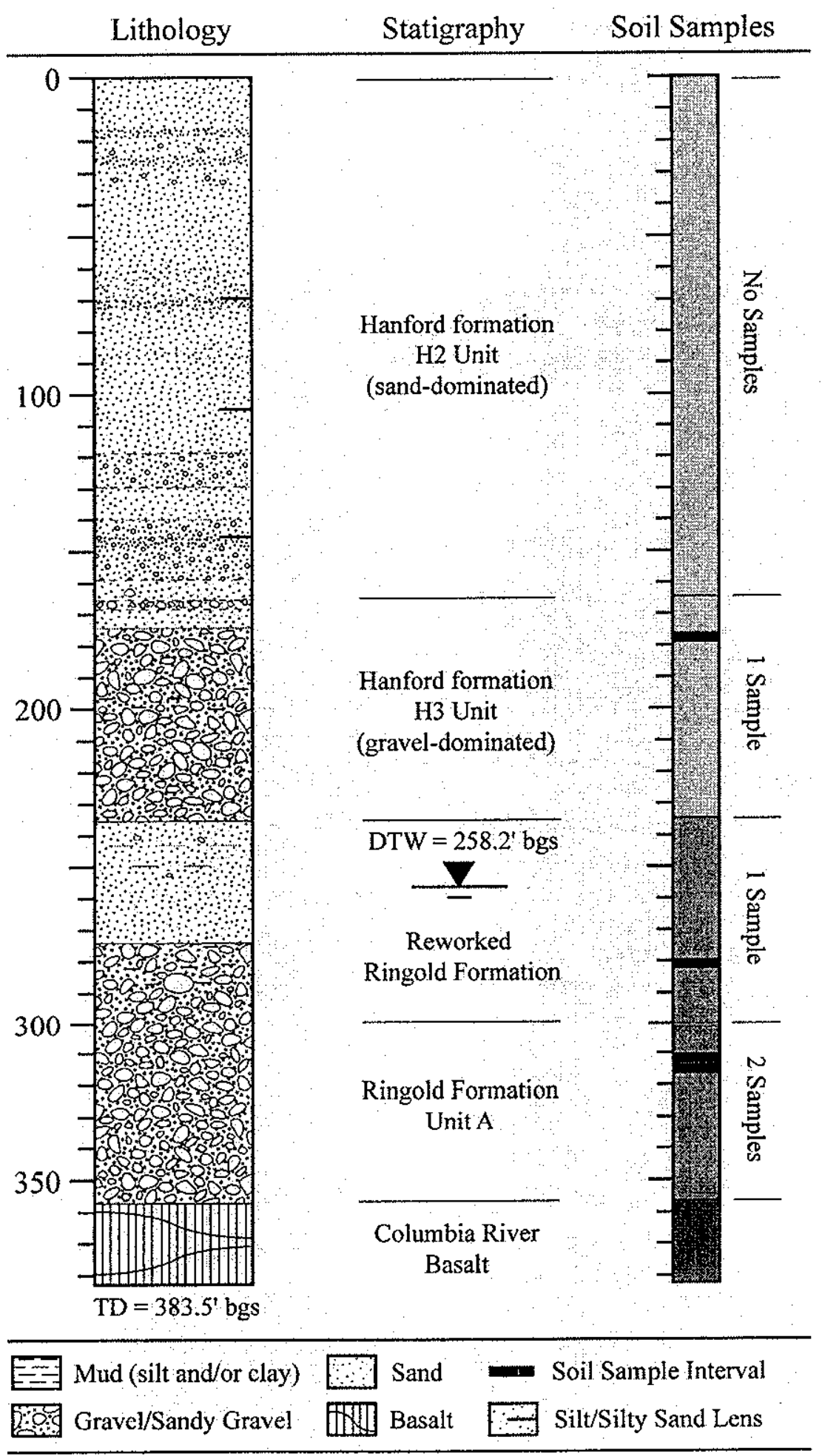


WMP-32119, Rev. 0

This page intentionally left blank. 
WMP-32119, Rev. 0

\subsection{REFERENCES}

ASTM D4015-92, Standard Test Methods for Modulus and Damping of Soils by the Resonant-Column Method. ASTM International, West Conshohocken, Pennsylvania.

ASTM D6913-04e1, Standard Test Methods for Particle-Size Distribution (Gradation) of Soils Using Sieve Analysis. ASTM International, West Conshohocken, Pennsylvania.

Comprehensive Environmental Response, Compensation and Liability Act of 1980 , (CERCLA) 42 USC 9601, et seq.

CP-GPP-EE-01-7.0, Rev. 1, "Geologic Logging Procedure," Fluor Hanford, Inc., Richland, Washington.

CP-GPP-EE-02-14.1, "Drilling, Remediating, and Decommissioning Resource Protection Wells, and Geotechnical Soil Borings," Fluor Hanford, Inc., Richland, Washington

DOE/RL-2004-18. 2004. Waste Control Plan for the 200-PO-1 Operable Unit. U.S. Department of Energy Richland Operations Office, Richland, Washington.

Gardner MG, KD Reynolds, and DE Skoglie. 2006. Drilling Plan for the Waste Treatment Plant Seismic Test Boreholes Project. FS-RW-SWS-PN-005, Rev. 0, Duratek Federal Services, Richland, Washington.

HNF-EP-0063, 2004, Hanford Site Solid Waste Acceptance Criteria, Rev. 10, Fluor Hanford, Inc., Richland, Washington.

HNF-PRO-10863, 2002, "Notebooks and Logbooks," Rev. 0, Fluor Hanford, Inc., Richland, Washington.

RCW 70.105, "Hazardous Waste Management," Revised Code of Washington, as amended

UTSD RCTS GR06-4. 2006. Test Procedures and Calibration Documentation Associated with the RCTS and URC Tests at the University of Texas at Austin, Geotechnical Engineering Center, Civil Engineering Department, The University of Texas at Austin, Austin, Texas.

WAC 173-160, "Minimum Standards for Construction and Maintenance of Wells," Washington Administrative Code, as amended.

WAC 173-303, "Dangerous Waste Regulations," Washington Administrative Code, as amended 
WMP-32119, Rev. 0

This page intentionally left blank. 
WMP-32119, Rev. 0

APPENDIX A

BOREHOLE LOG

ENTRY BOREHOLE C4993

$A-i$ 
WMP-32119, Rev. 0
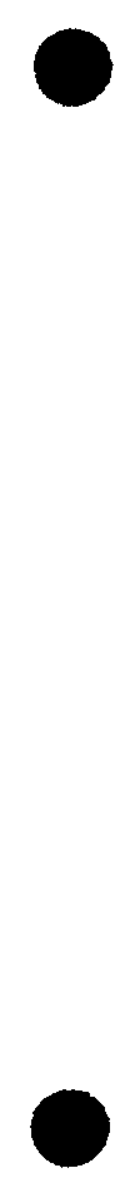

A-ii 


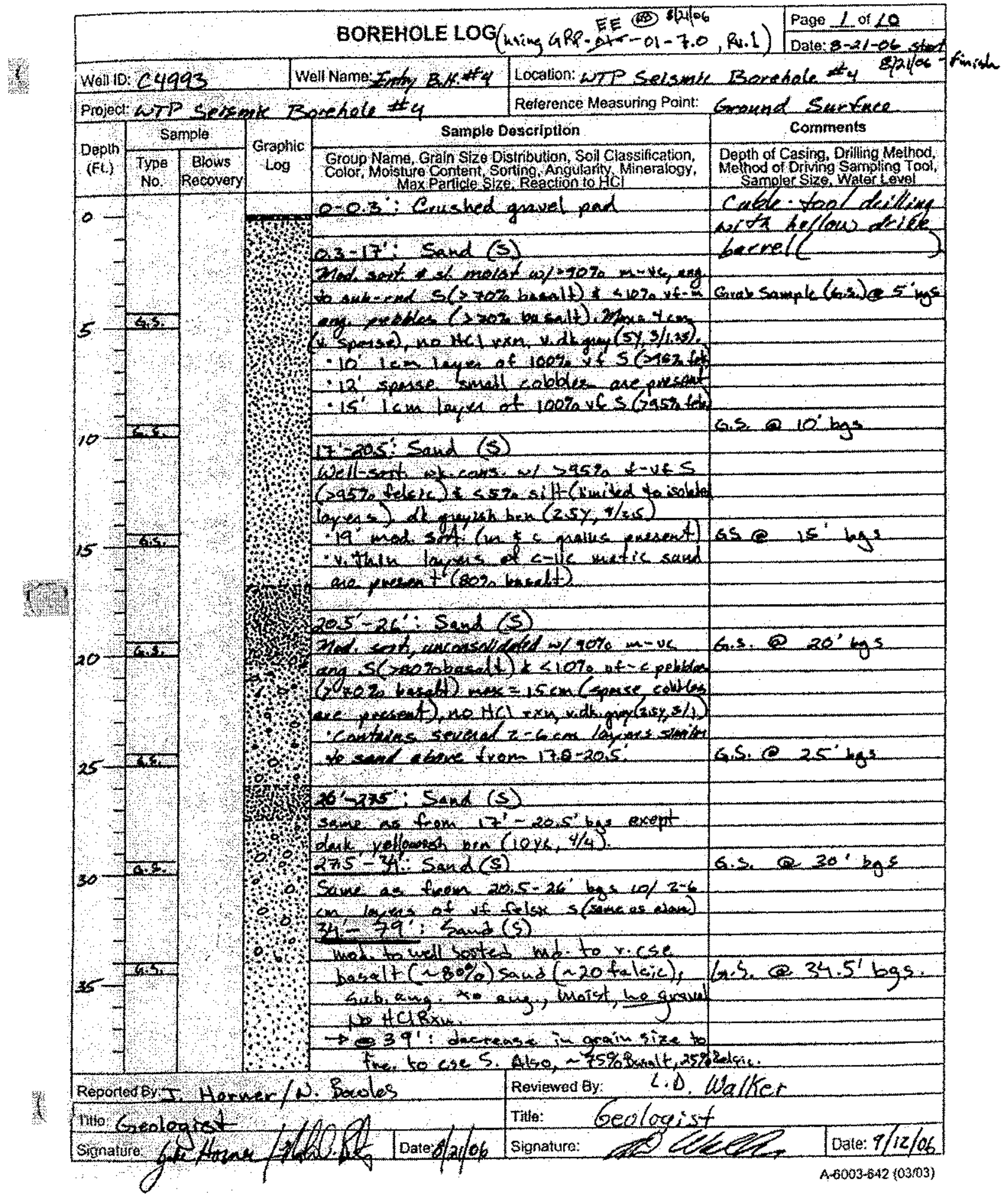




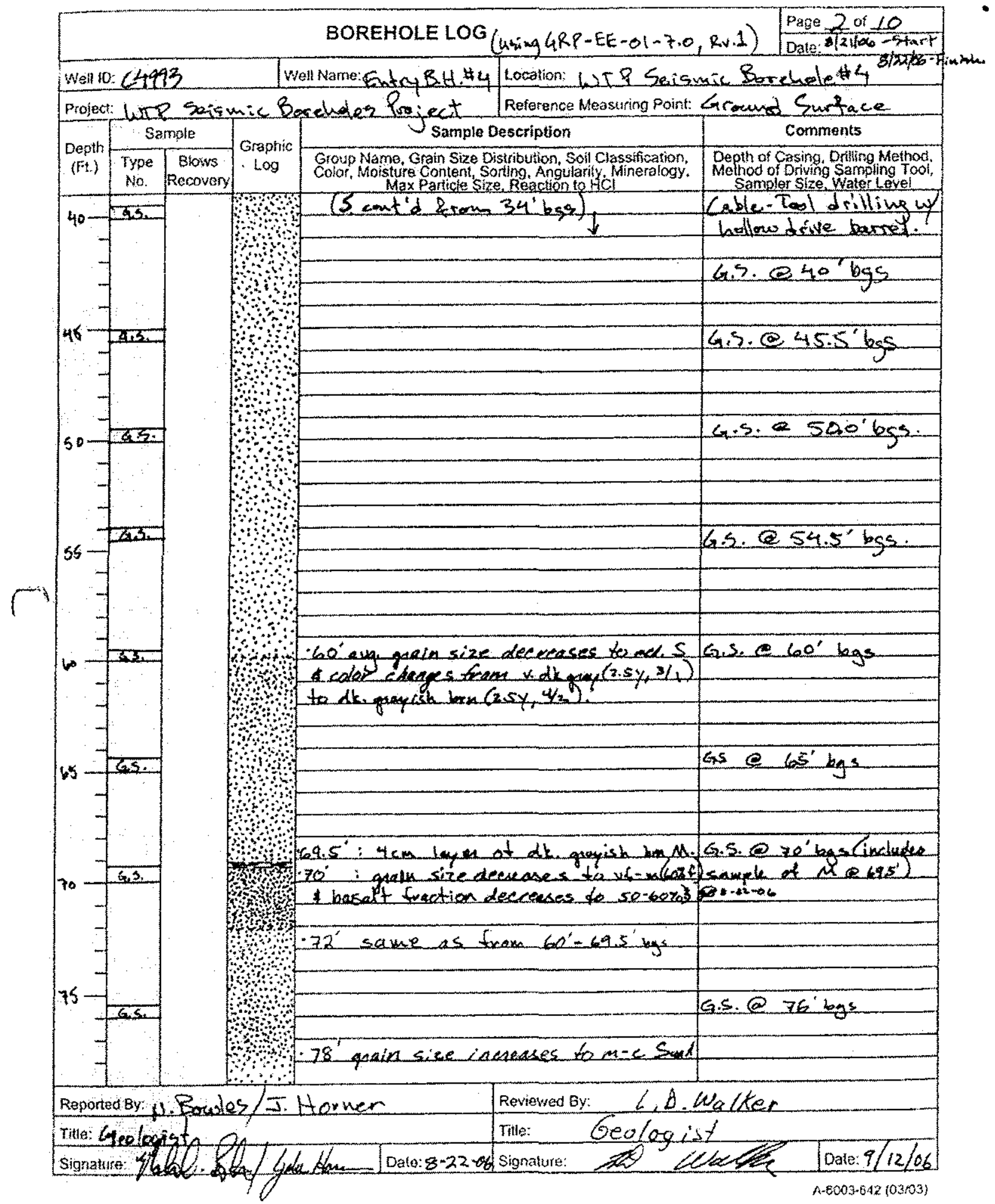




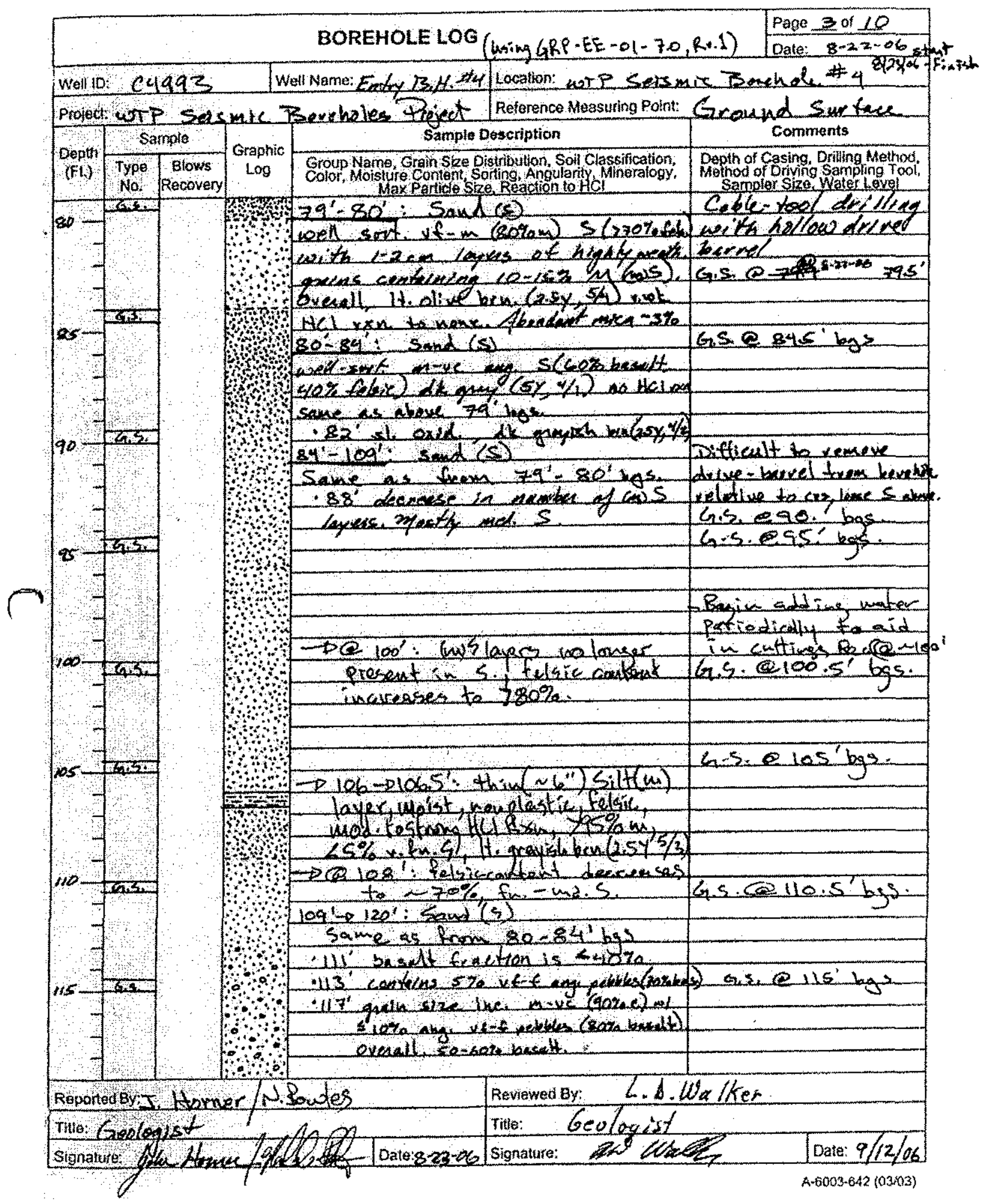




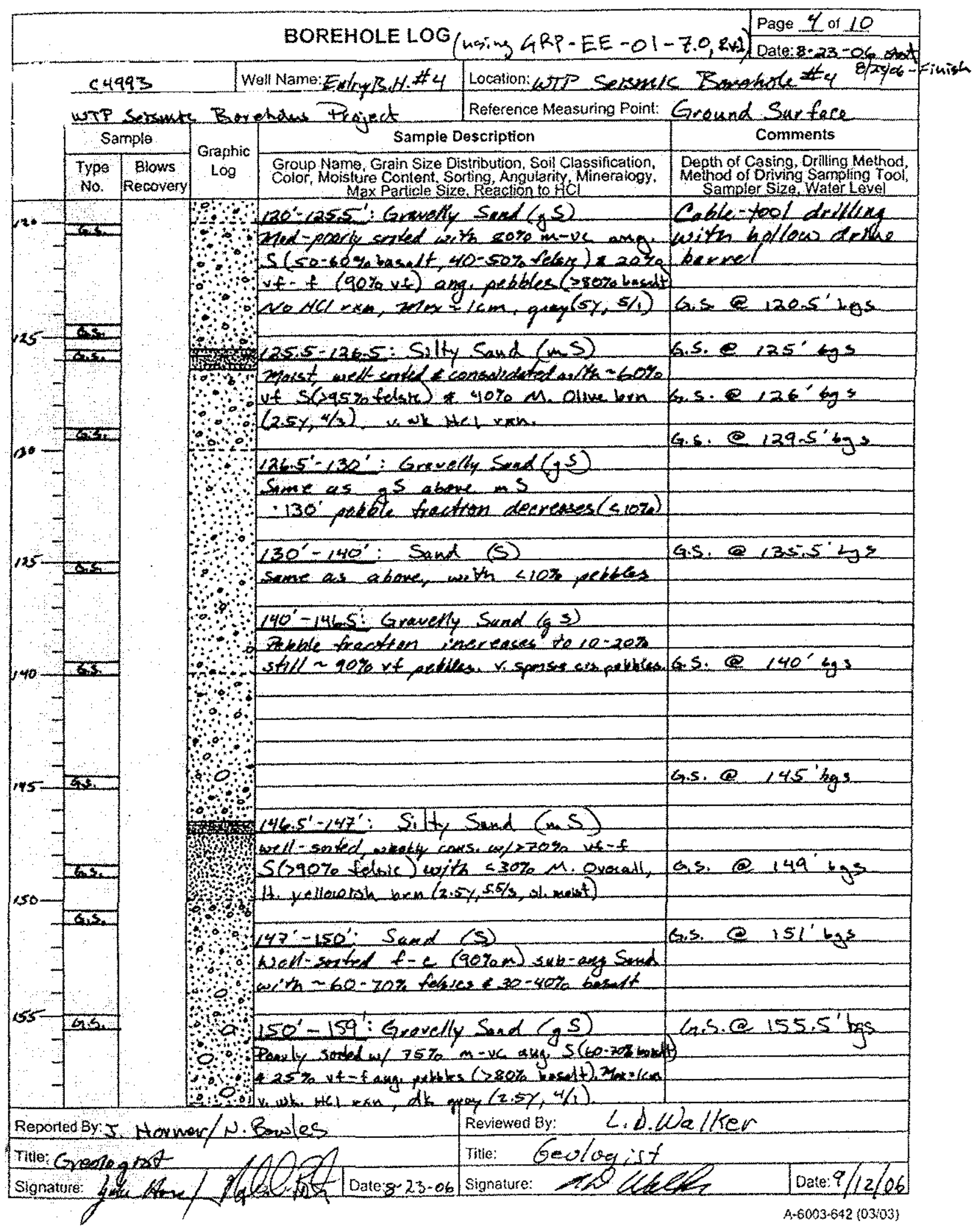




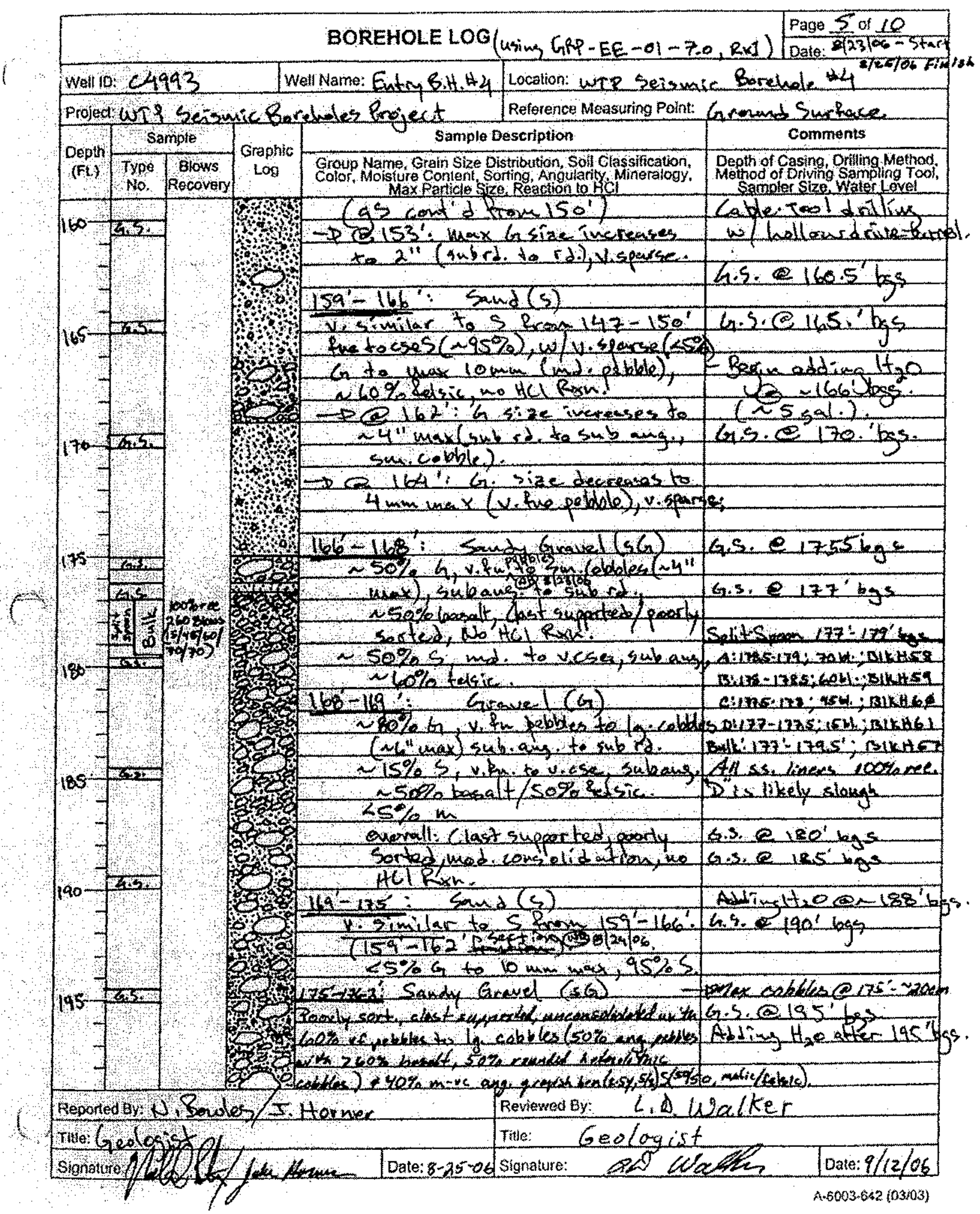


WMP-32119, Rev. 0

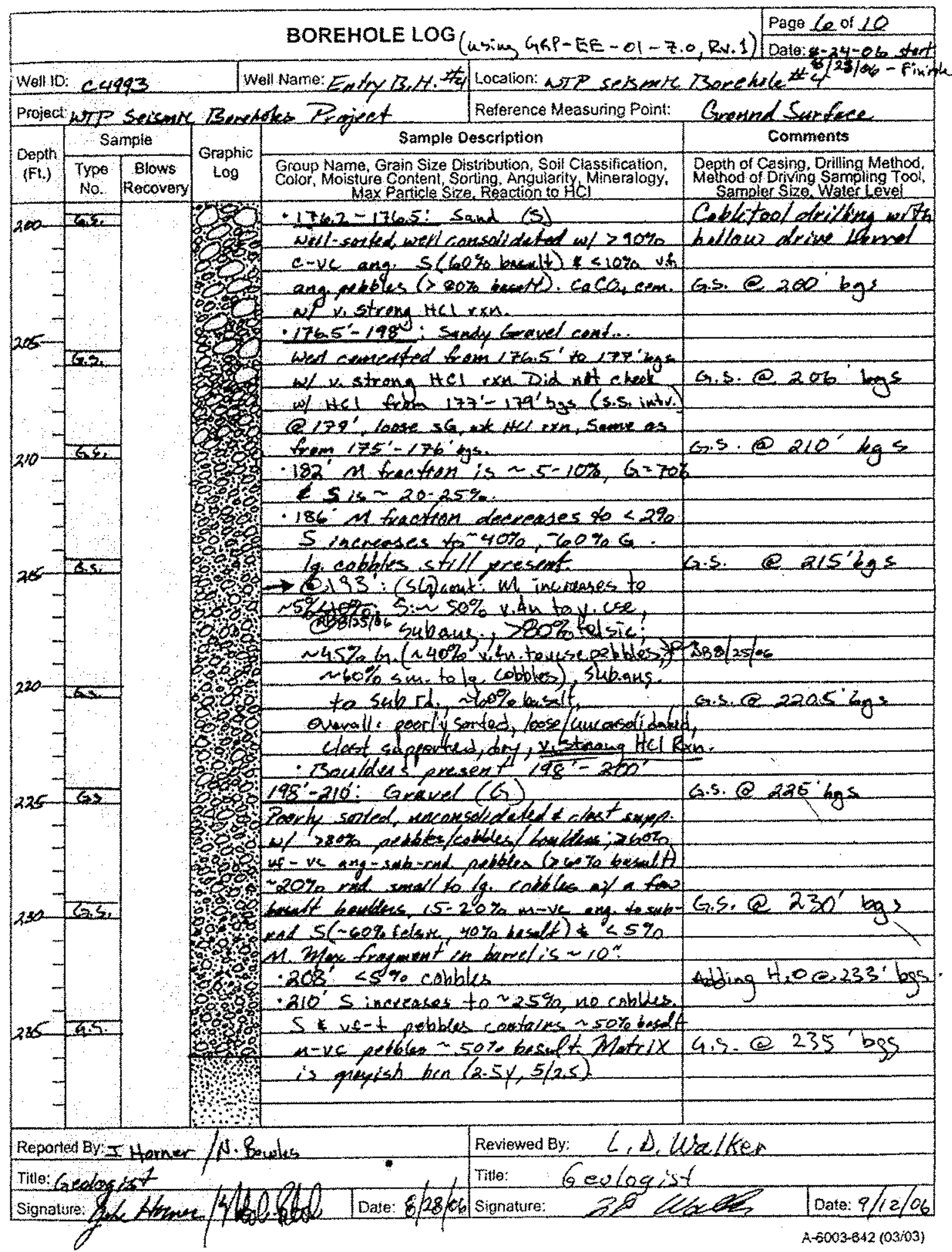




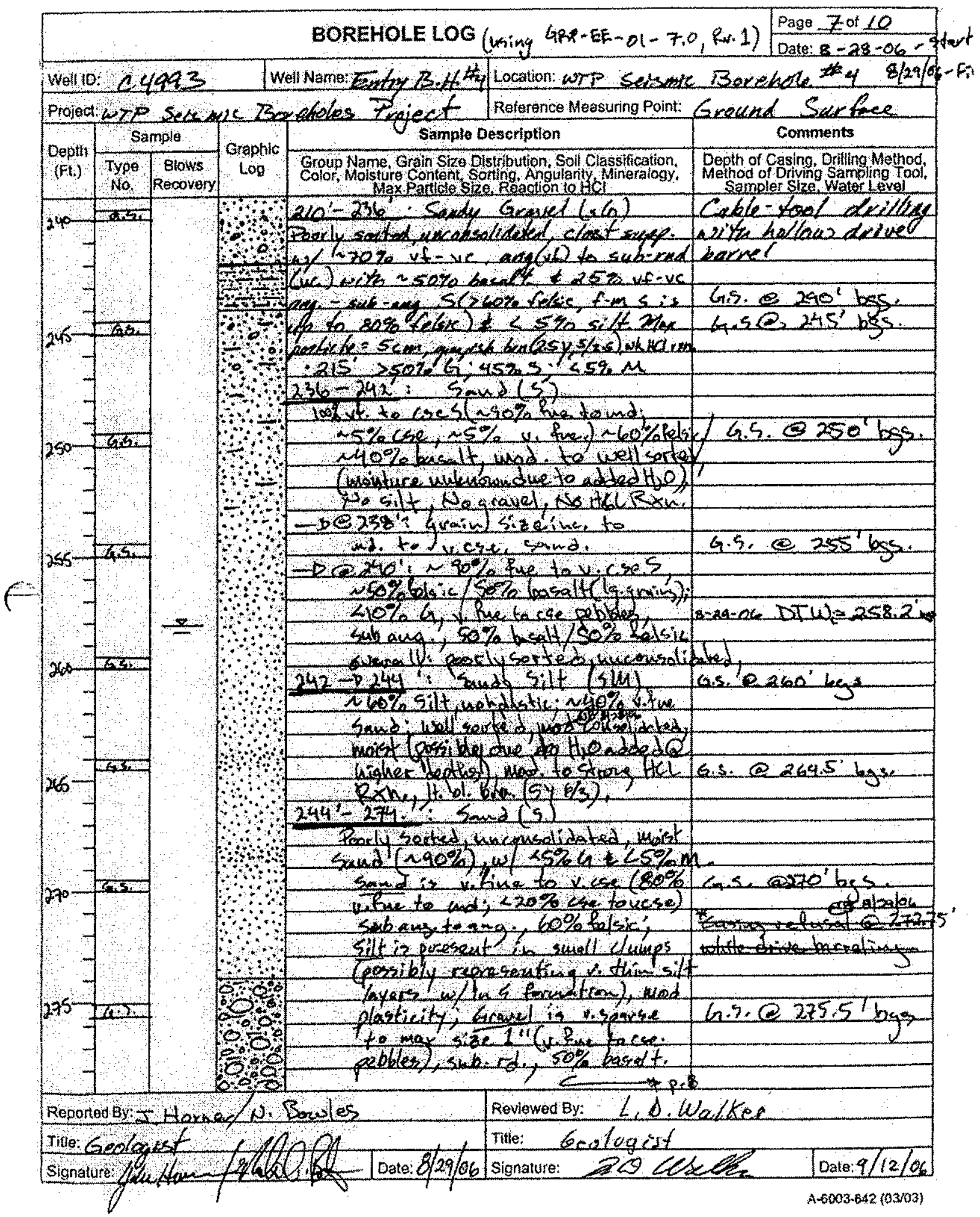




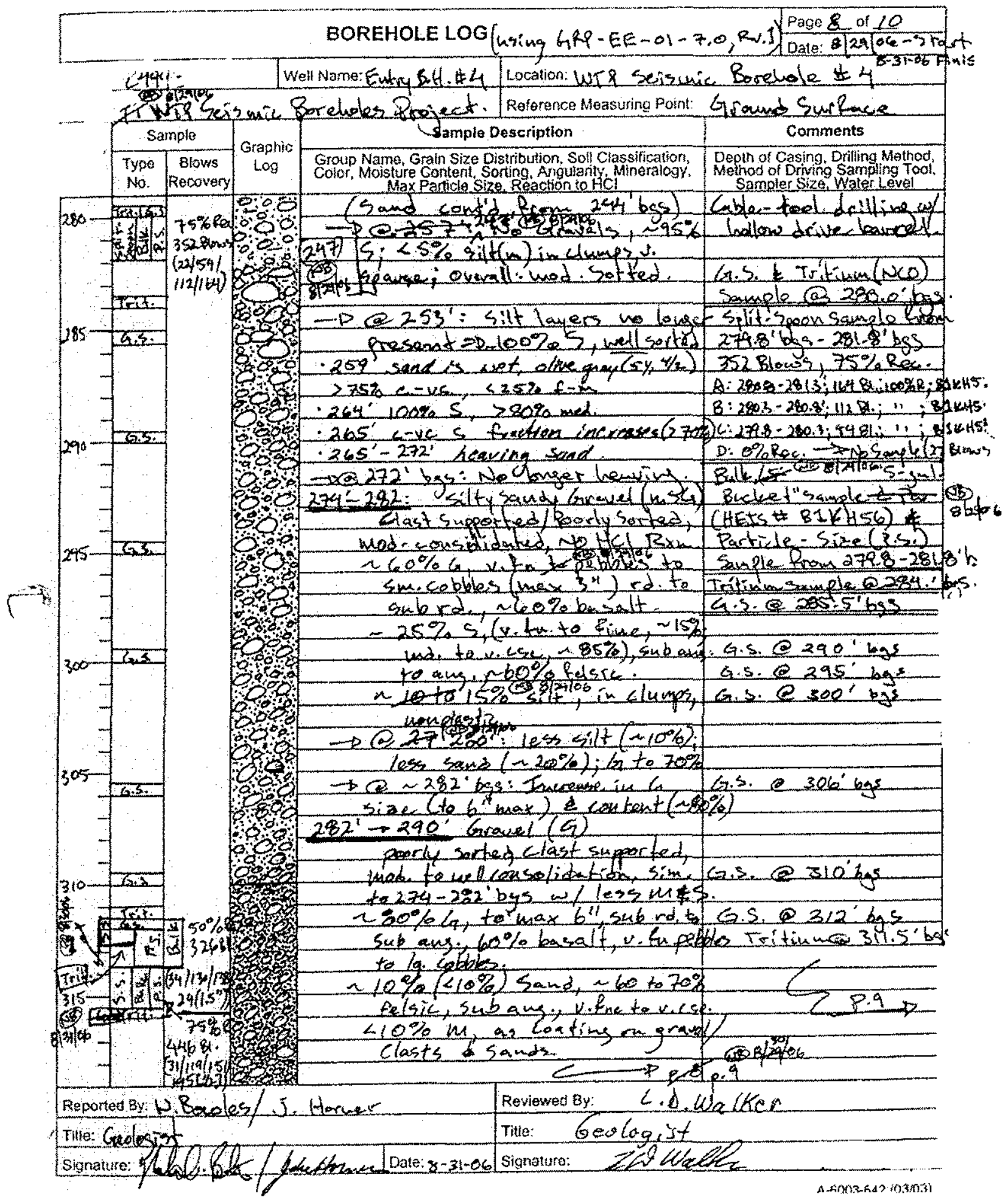


WMP-32119, Rev. 0

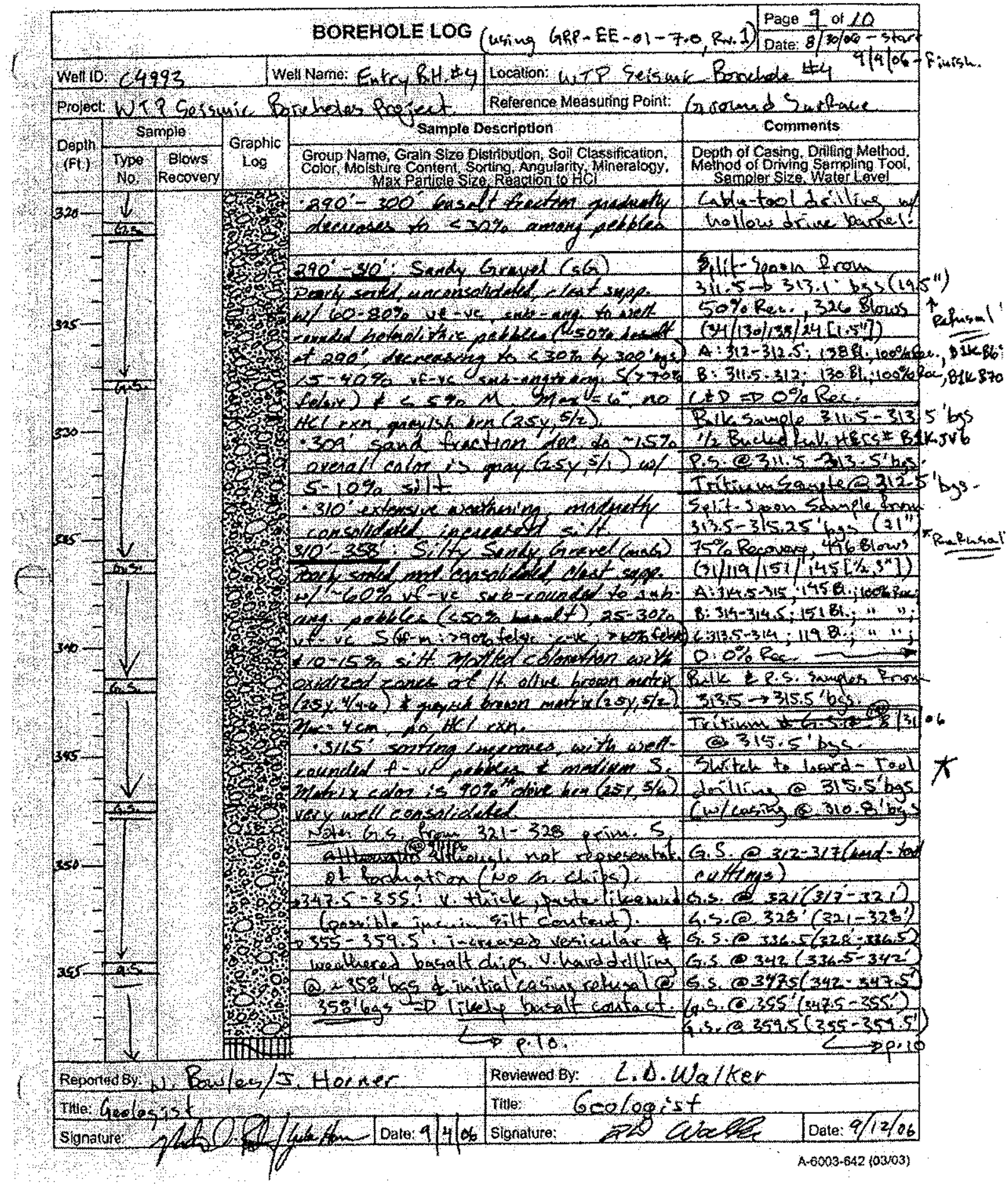


WMP-32119, Rev. 0

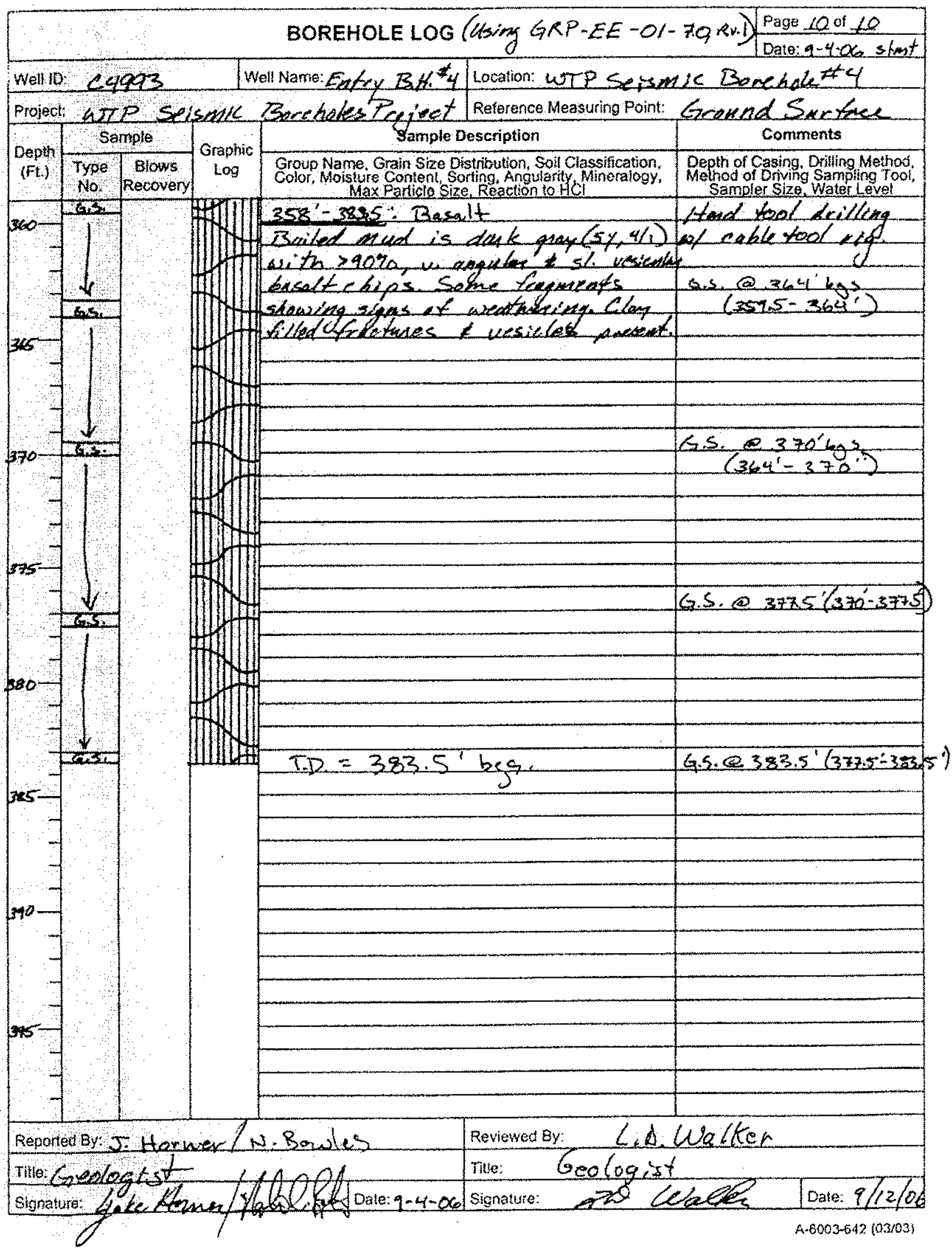


WMP-32119, Rev. 0

APPENDIX B

BOREHOLE LOG

ENTRY BOREHOLE C4996 
WMP-32119, Rev. 0
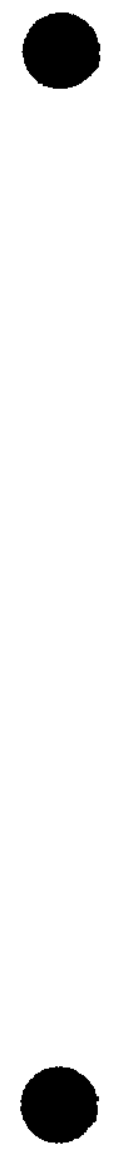

B-ii 
WMP-32119, Rev. 0

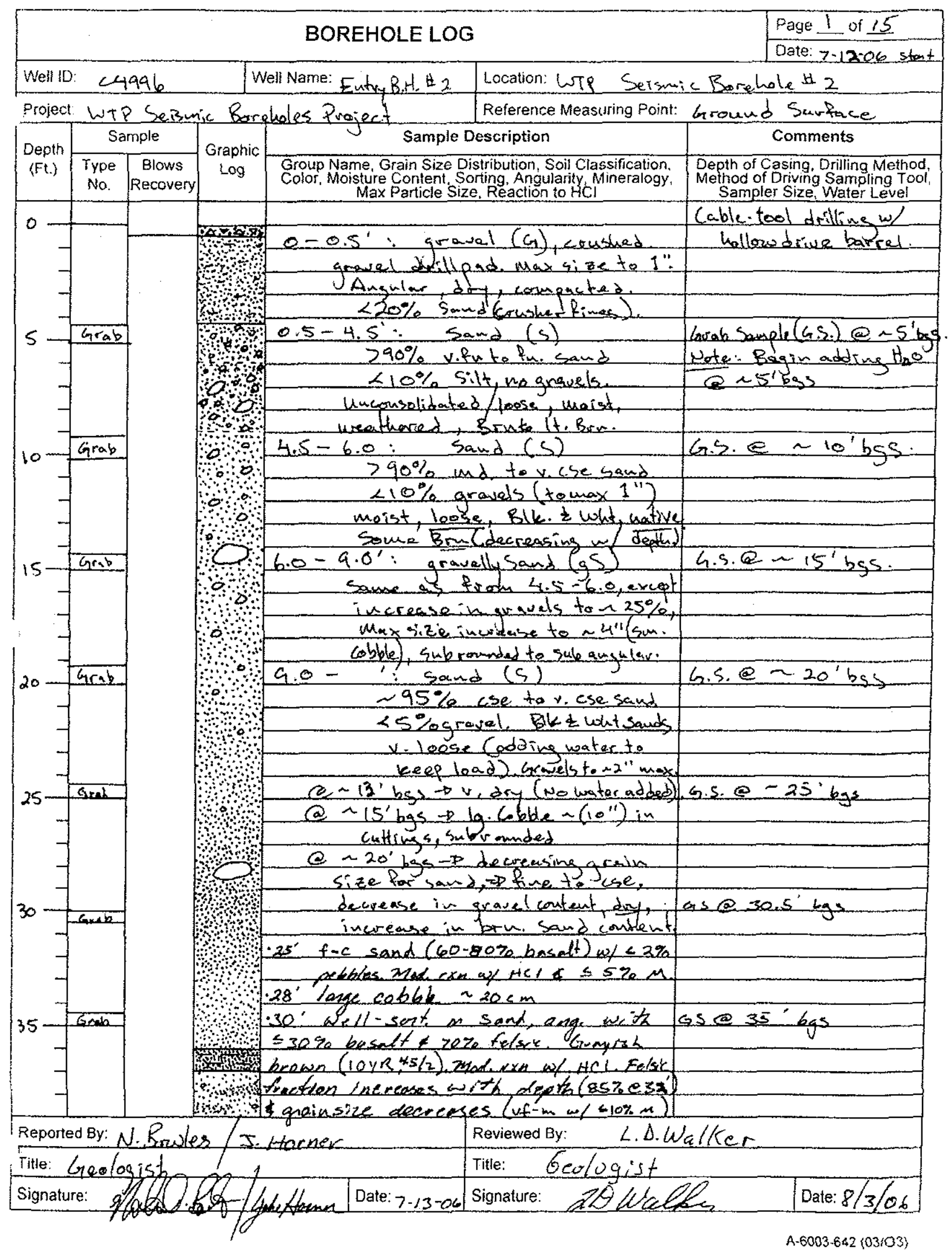




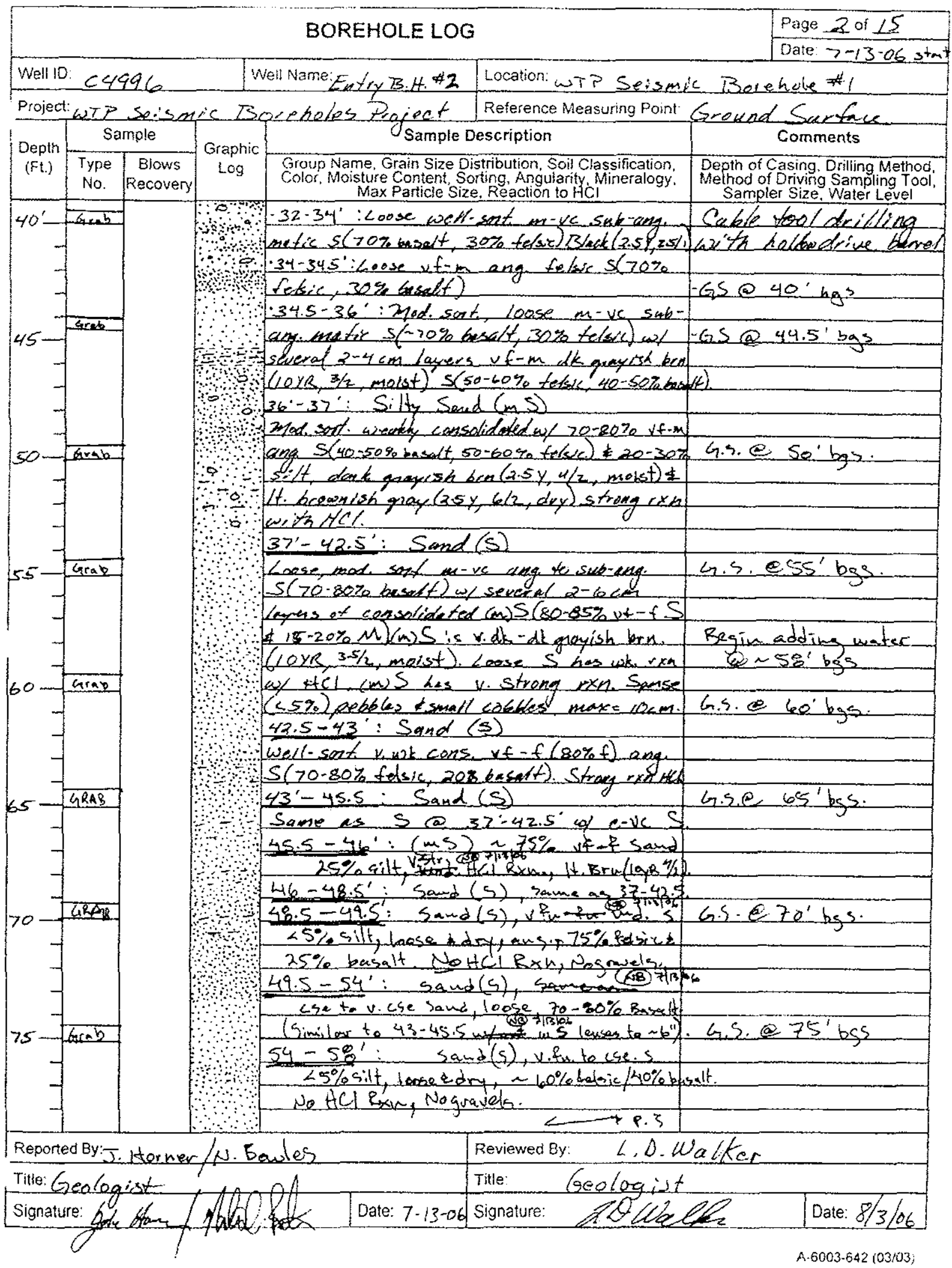


WMP-32119, Rev. 0

\section{BOREHOLE LOG}

\begin{tabular}{|c|c|c|}
\hline Well 10: 14996 & Well Name: Eutra \&.t. H2 & Location: wip Serzmic Bovehole \# $2^{7 / 16 / 06-7}$ \\
\hline
\end{tabular}

Project: WTR Seismic Bexeholes Preject. $\quad$ Reierence Measuring Point: Ground Sur face

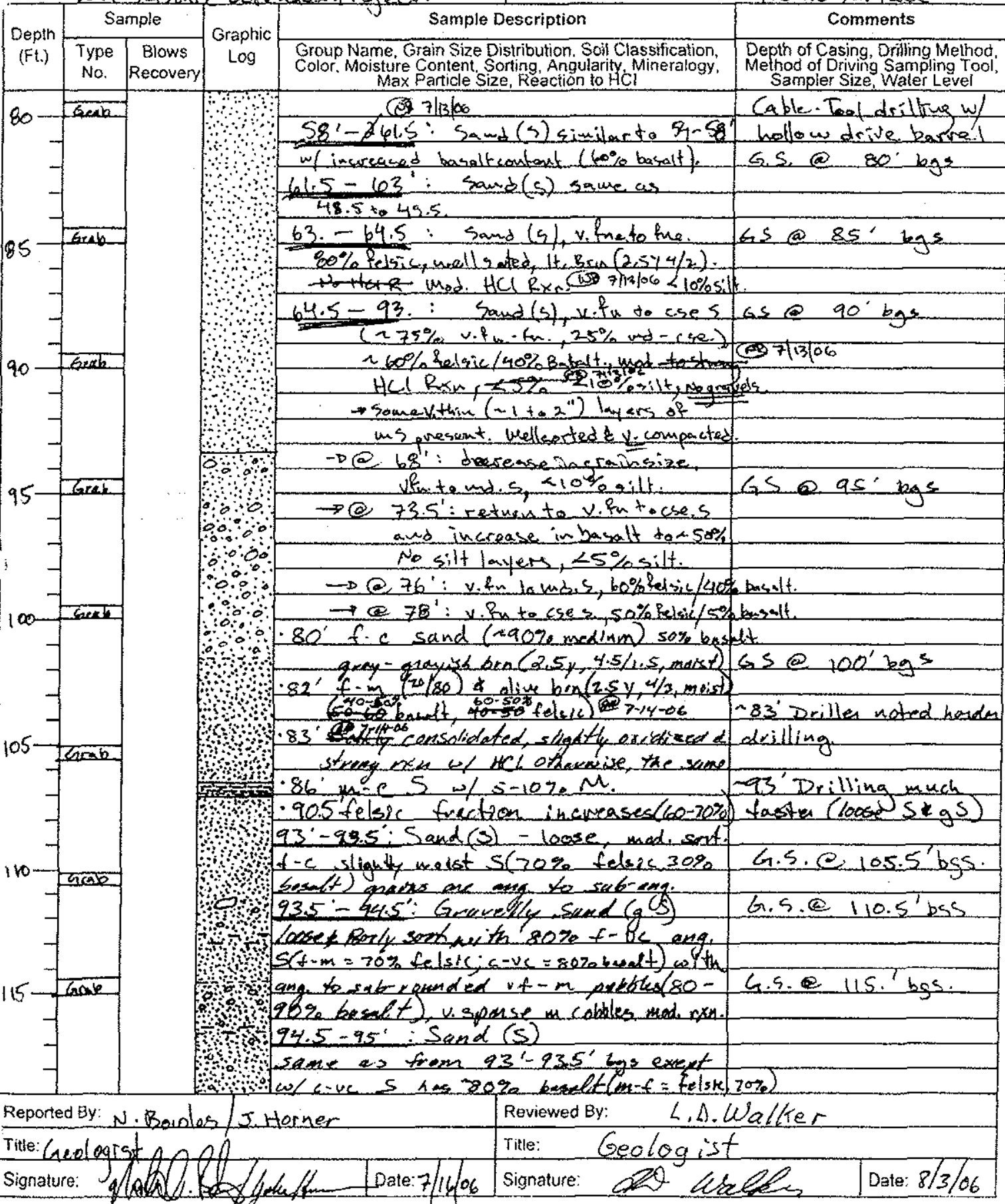

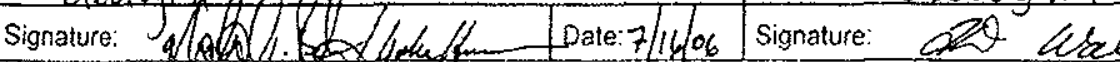


WMP-32119, Rev. 0

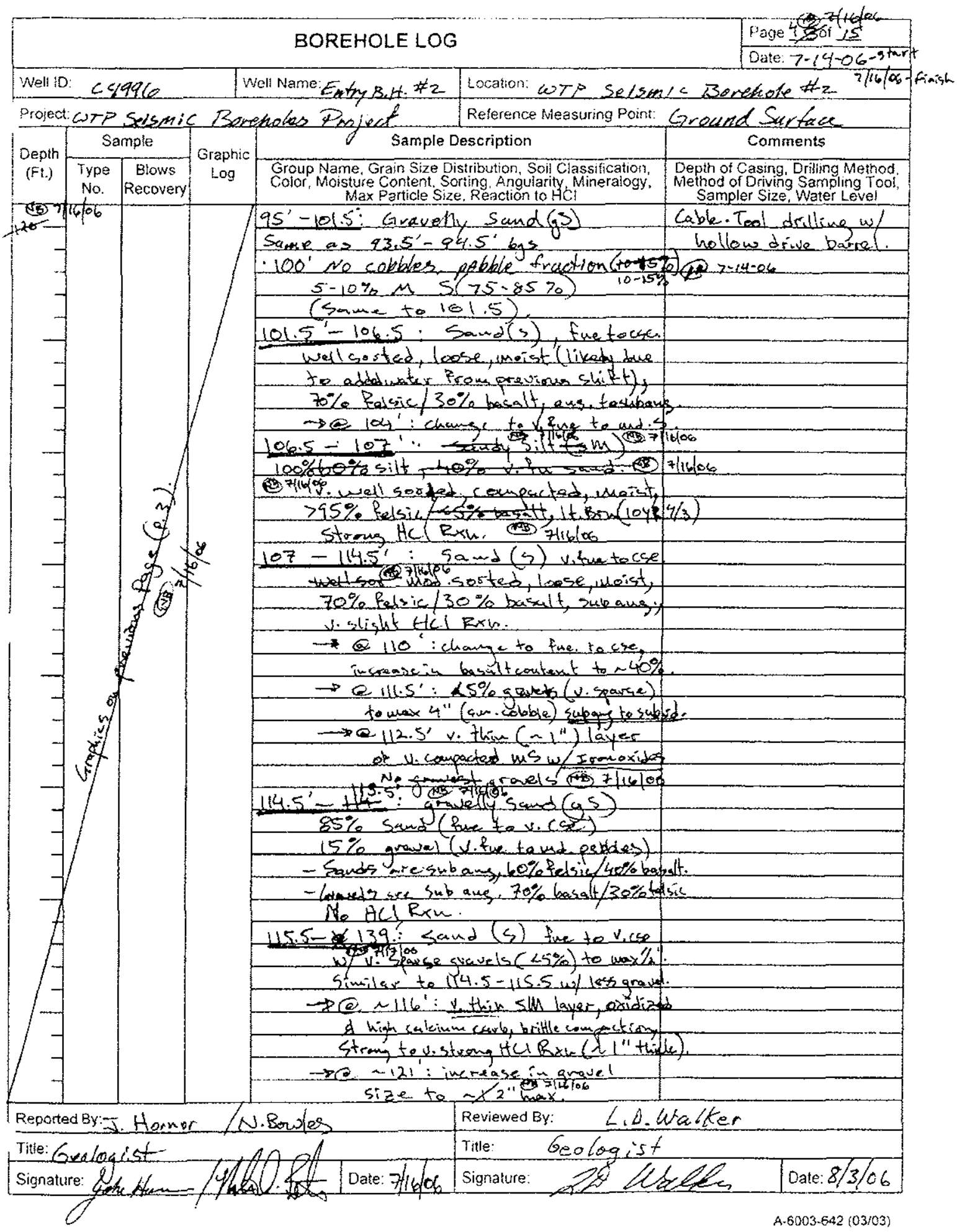




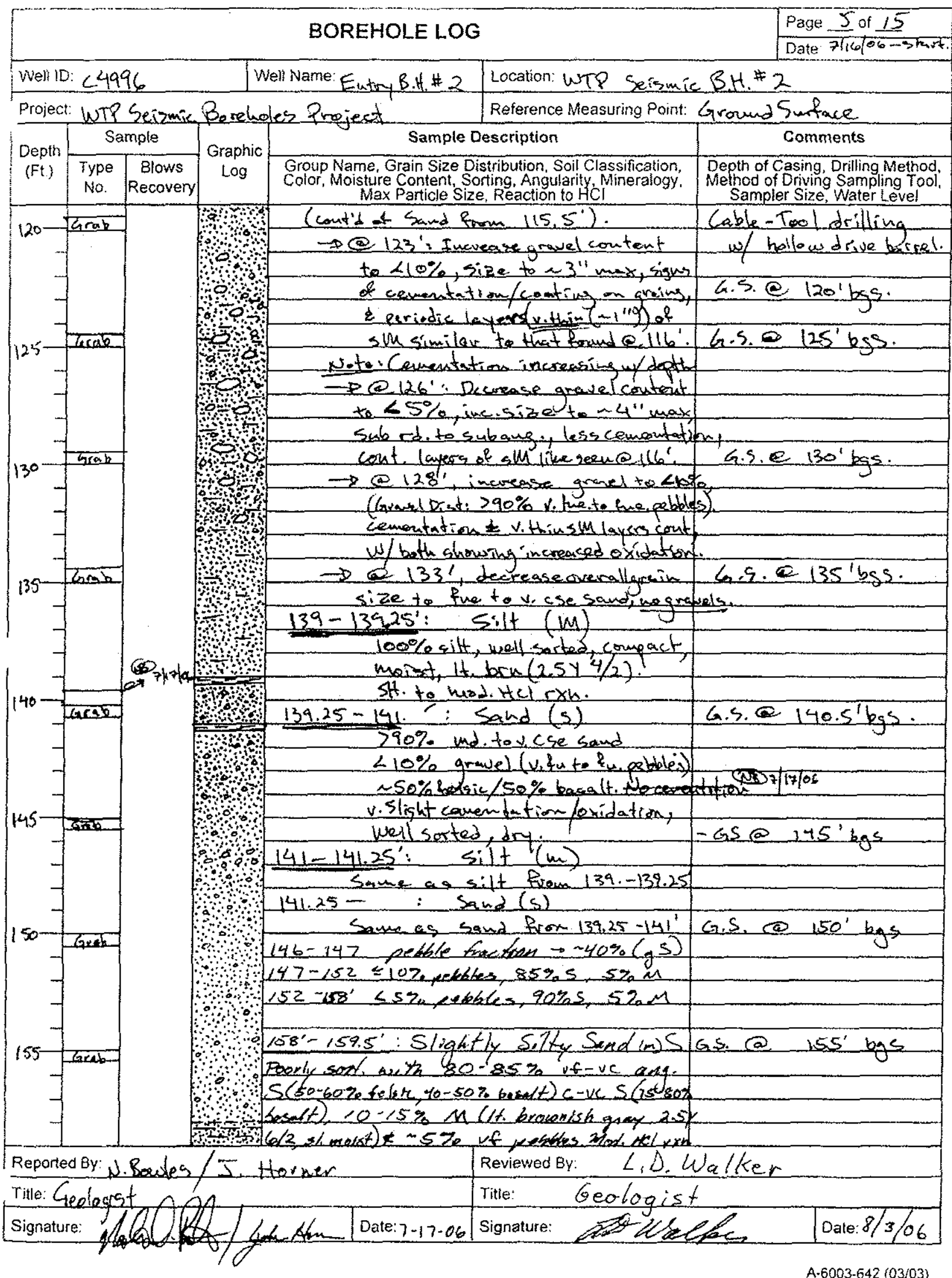




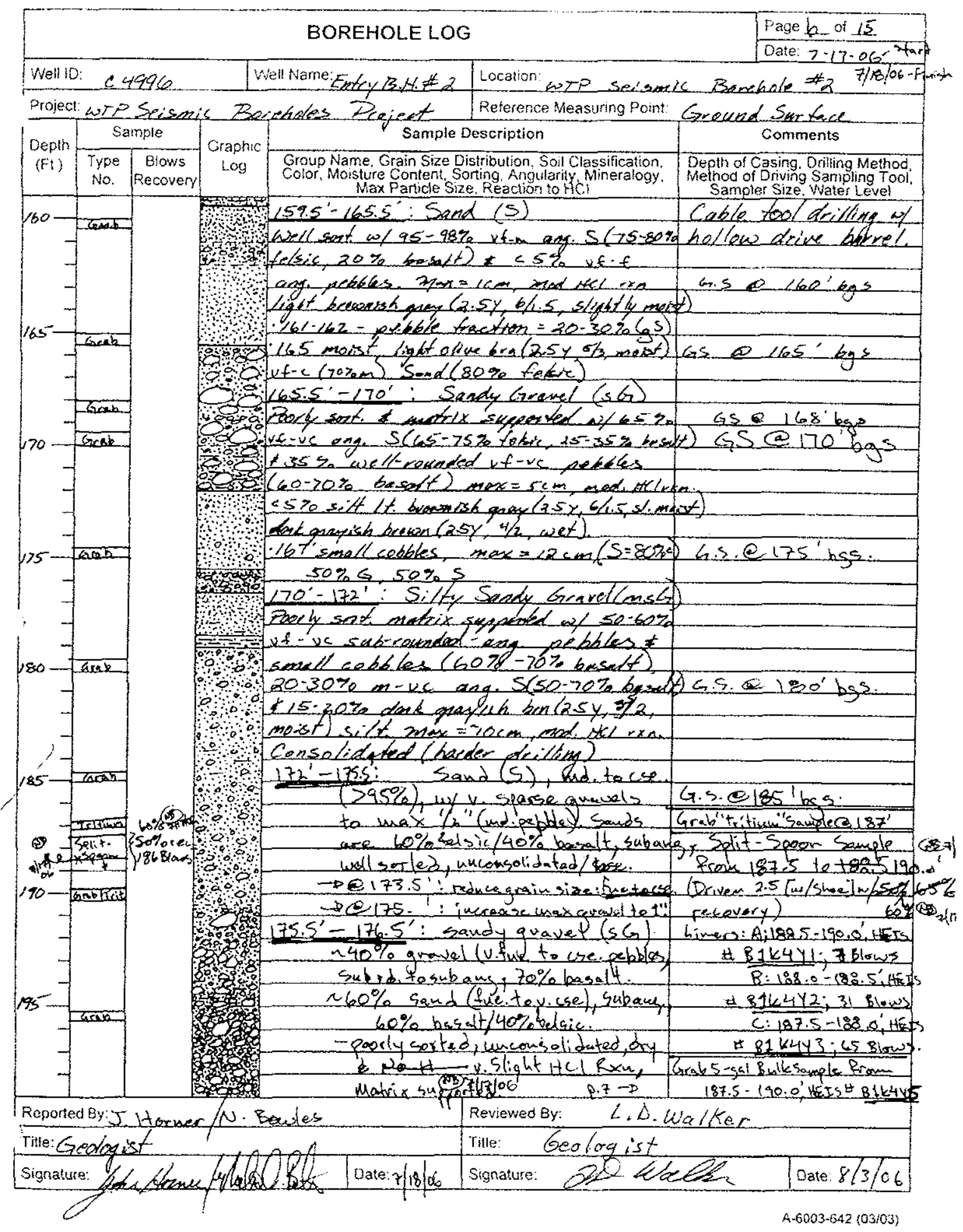


WMP-32119, Rev. 0

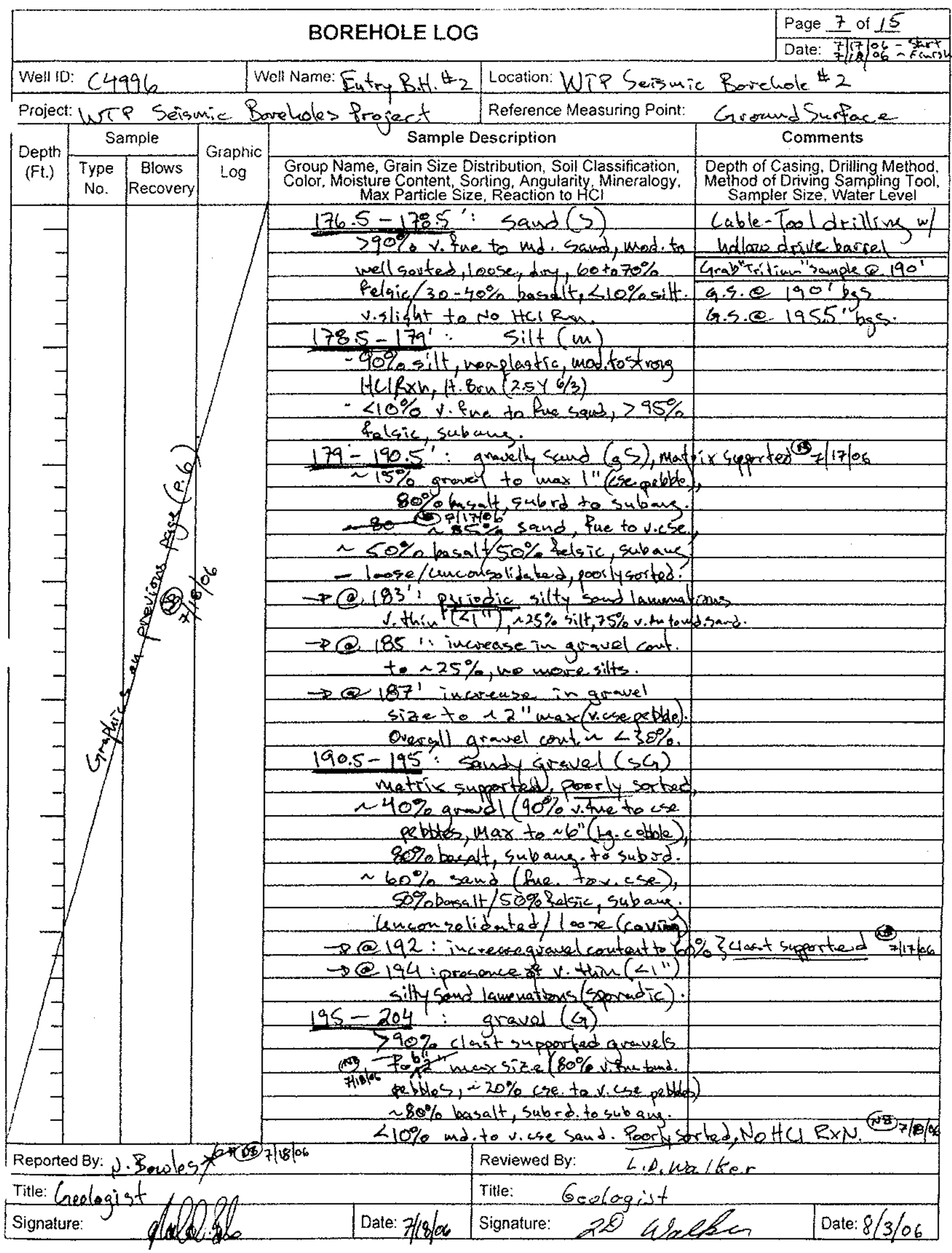

A-6003-642 (03/03) 
WMP-32119, Rev. 0

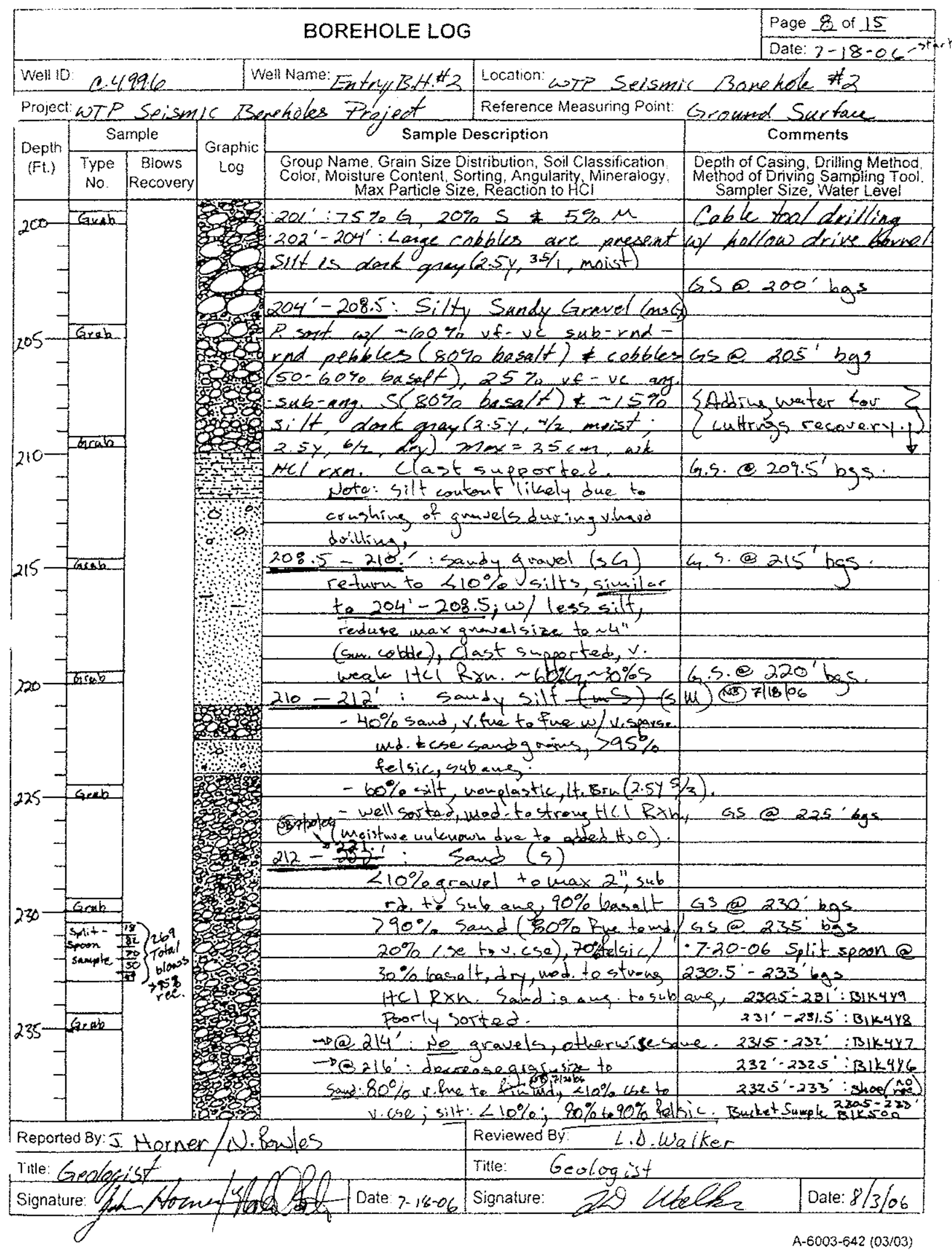


WMP-32119, Rev. 0

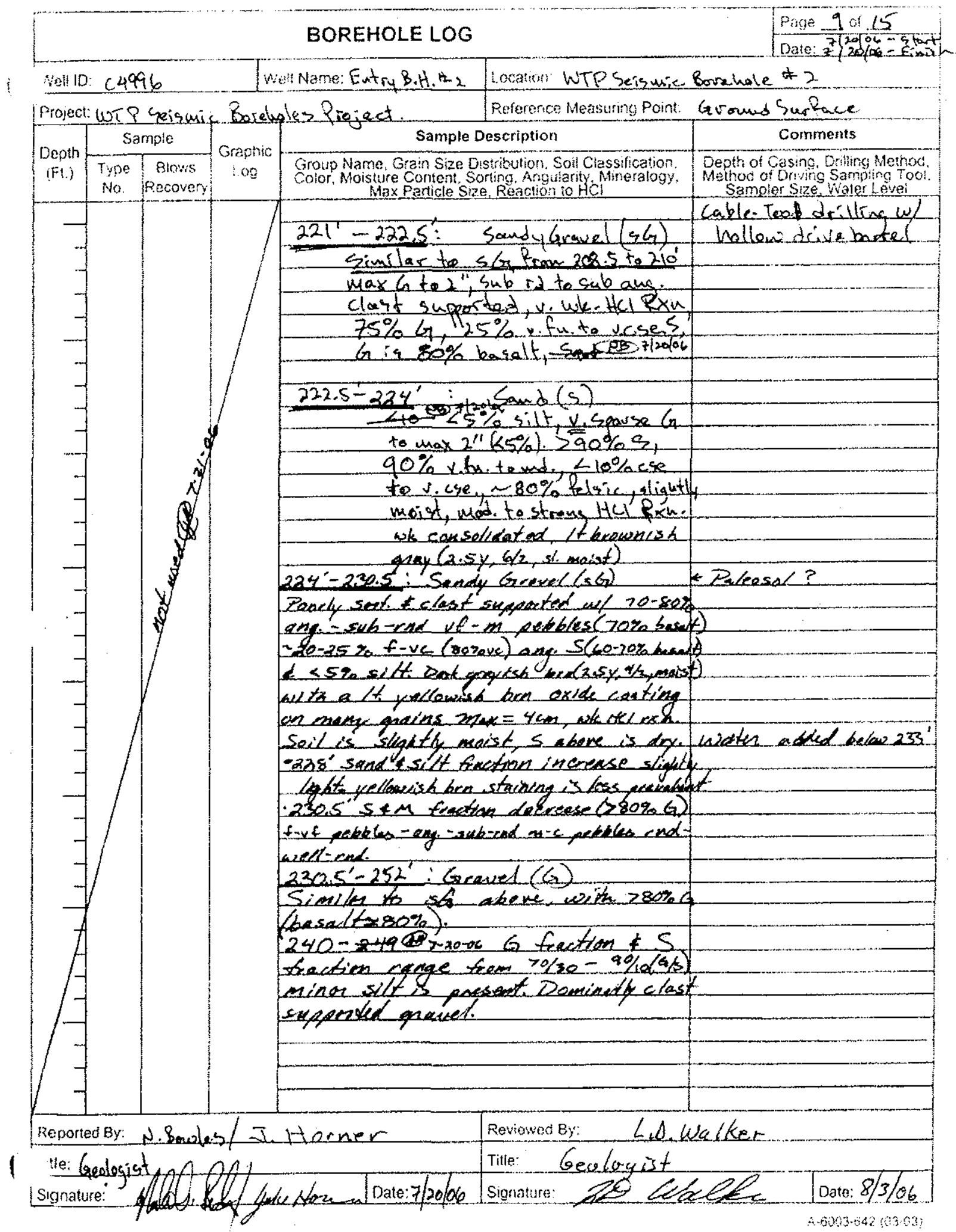


WMP-32119, Rev. 0

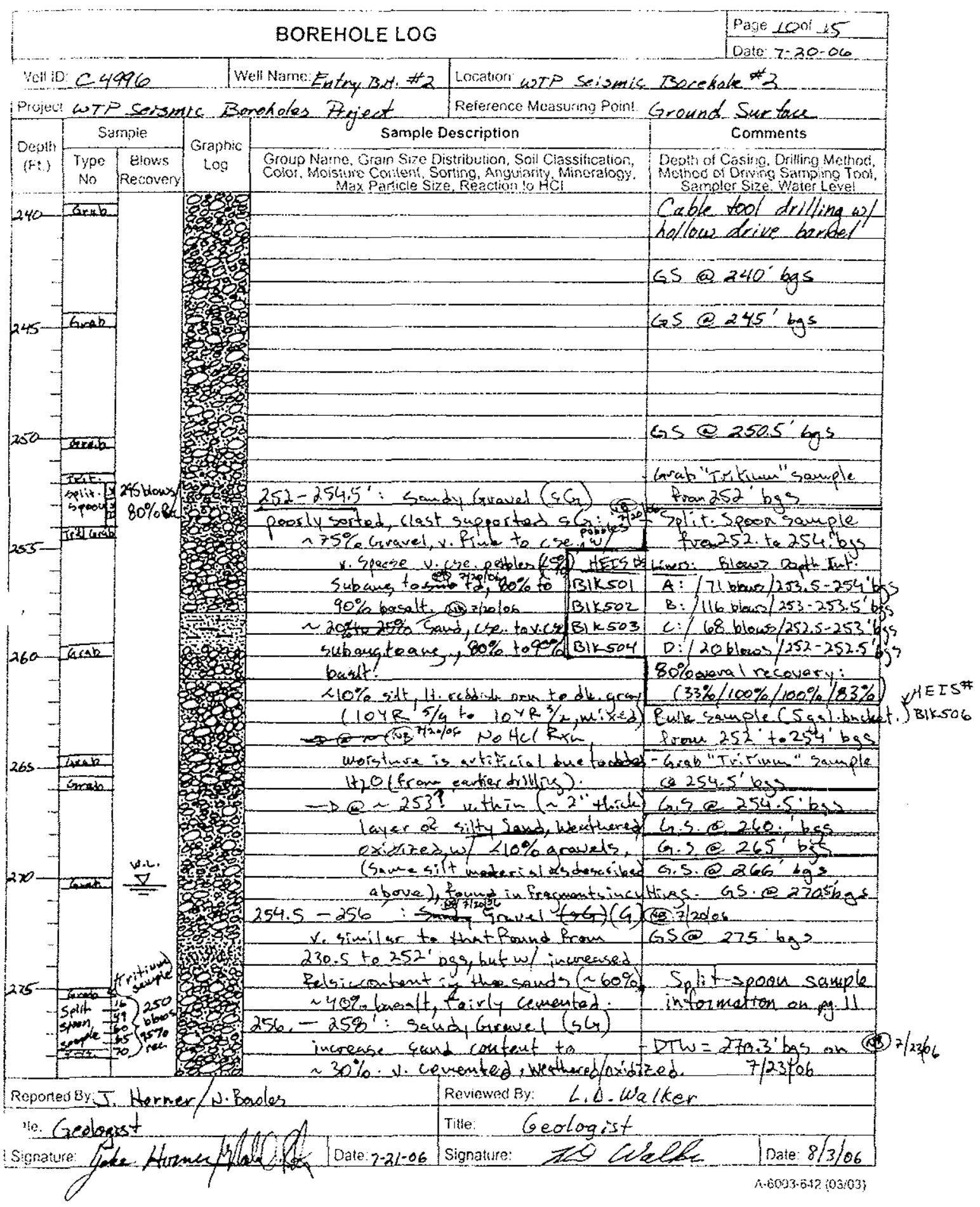


WMP-32119, Rev. 0

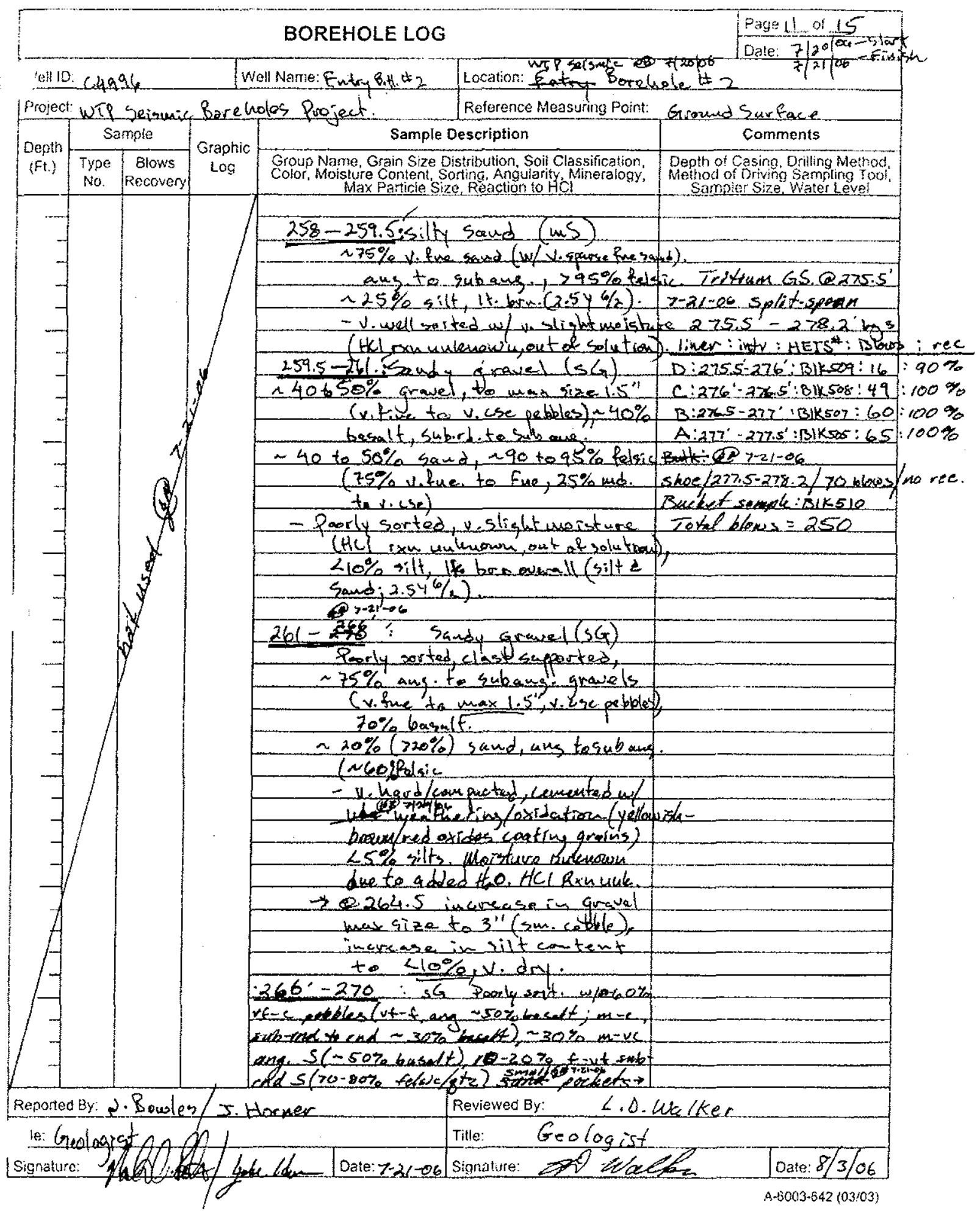




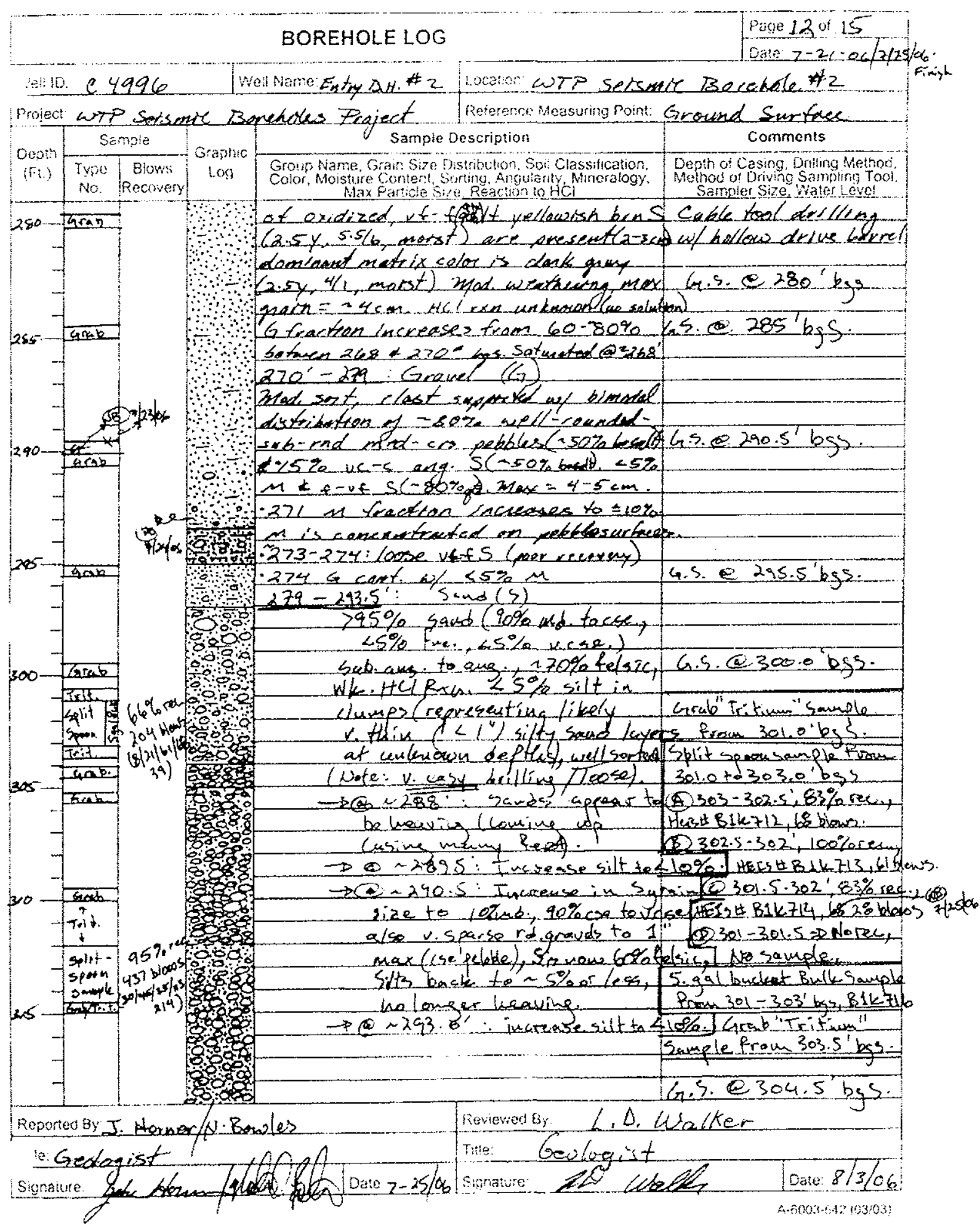


WMP-32119, Rev. 0

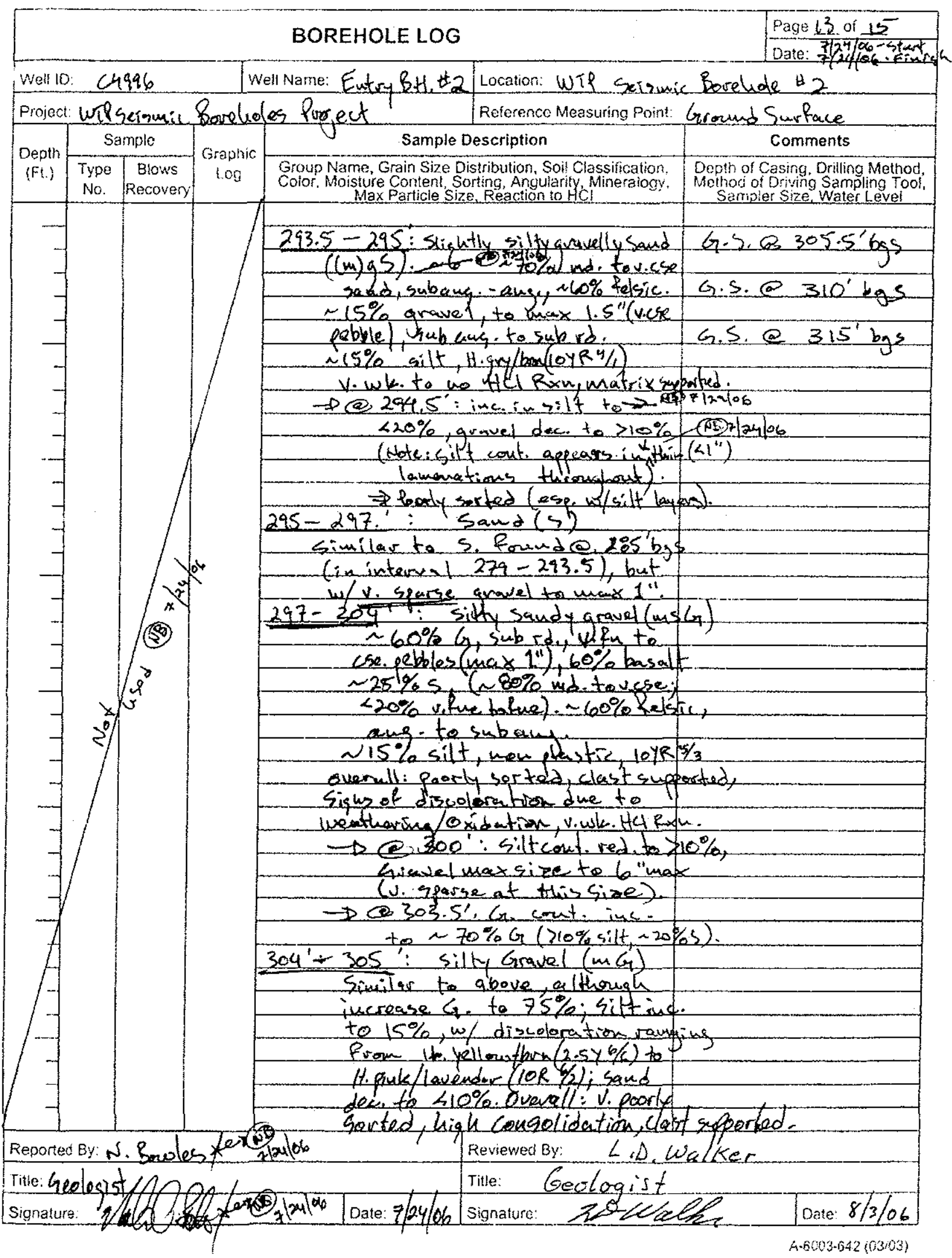


WMP-32119, Rev. 0

BOREHOLE LOG

Nonio 04996

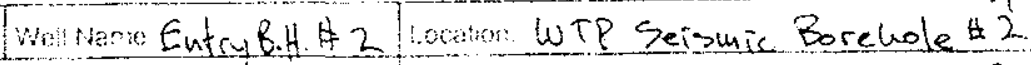

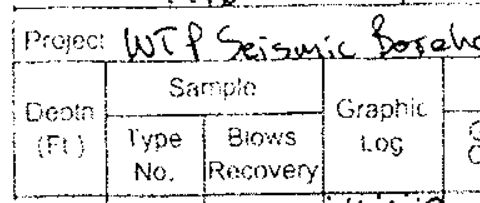
Taves

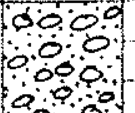
Project. Retenonce Mejsung Pont

Sample Description

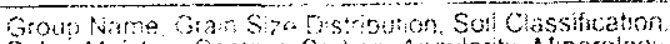

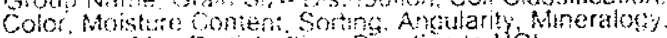
Mex Particle Sze Reaction to $\mathrm{HC}$

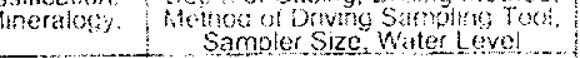

Giound Surface Comments

Gem on Casing, Doling Mewo

$305-3 / 2$ Gravel (G), v. Poorly Cable. Tool drílline

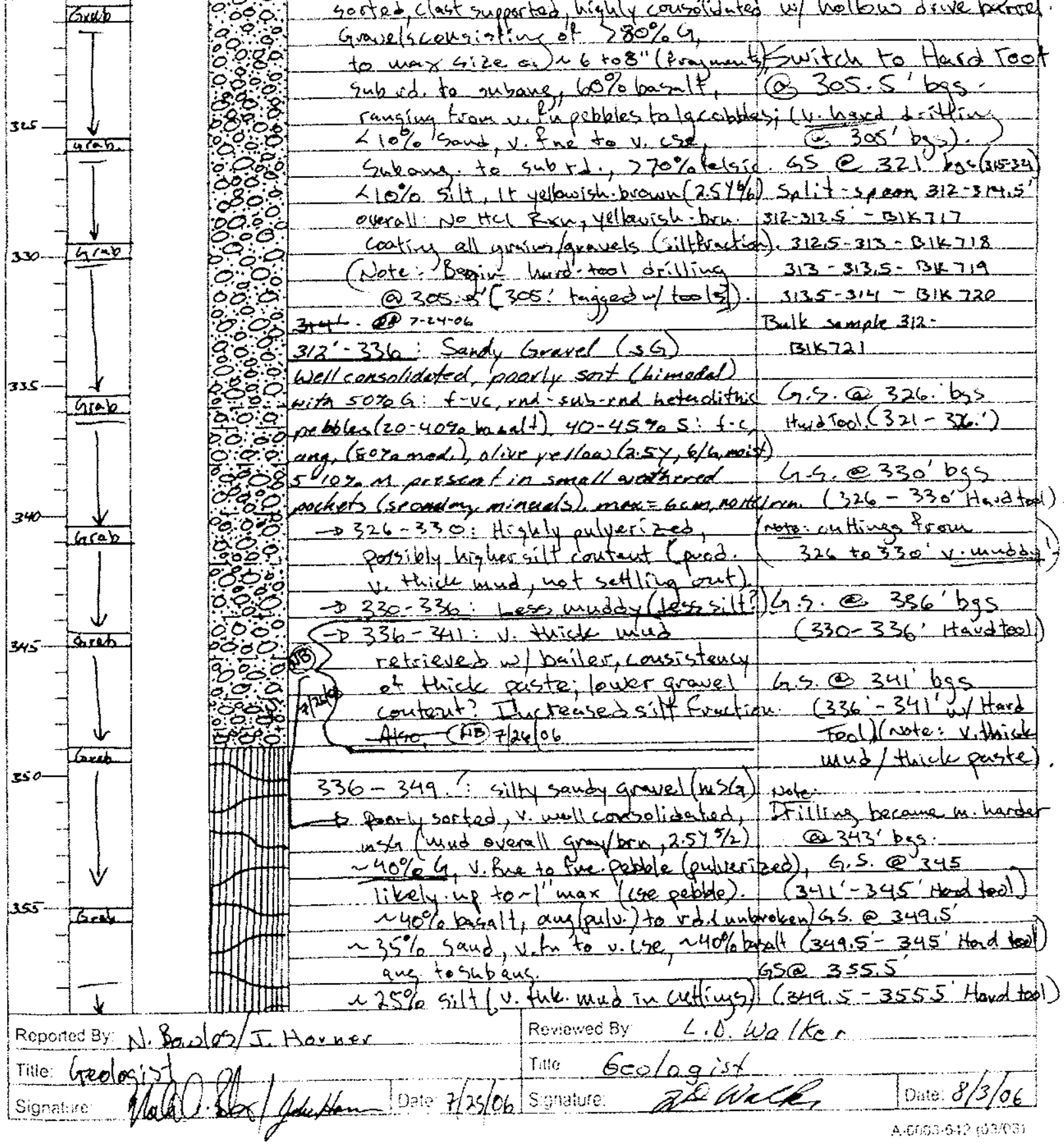




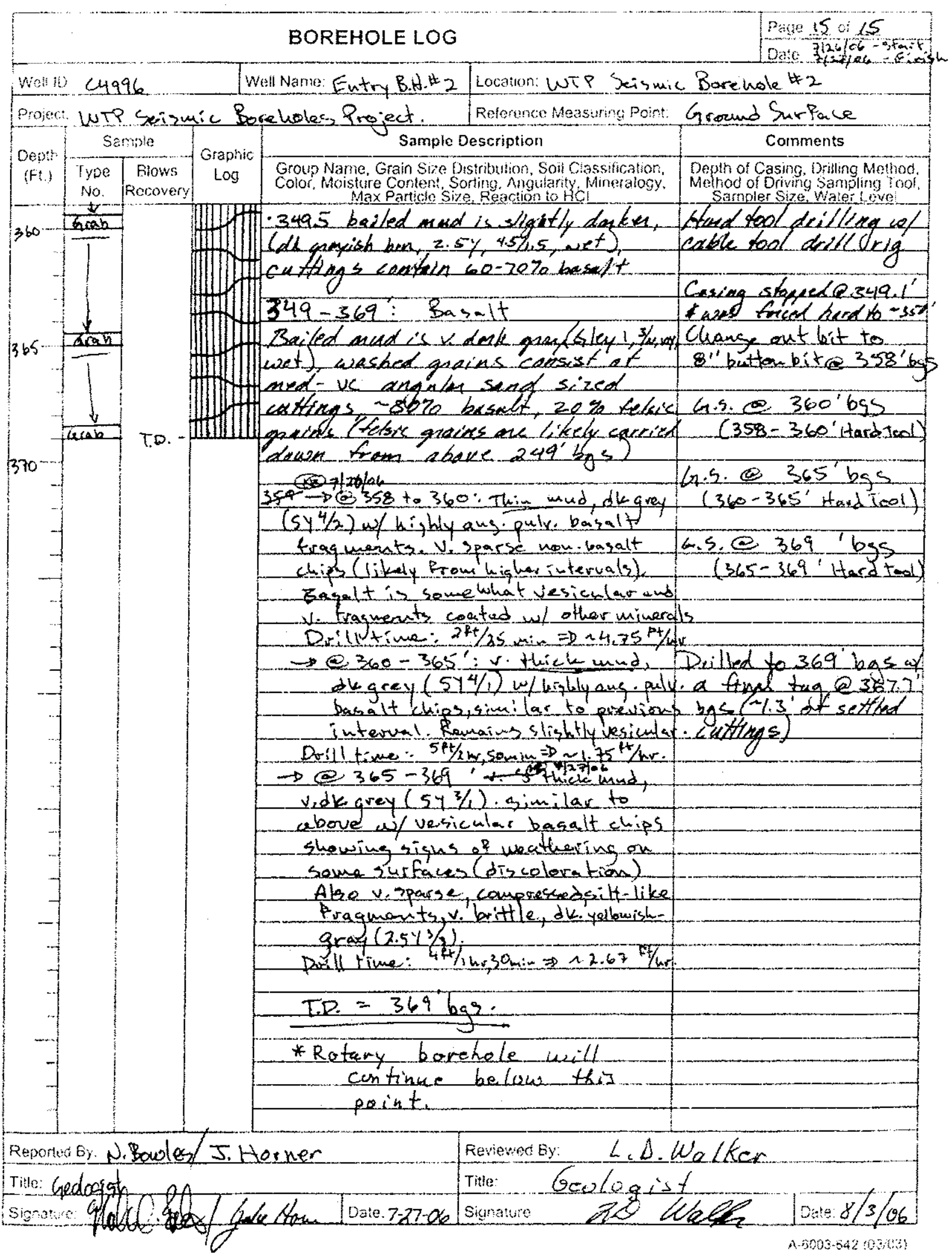


WMP-32119, Rev. 0

This page intentionally left blank 
WMP-32119, Rev. 0

\section{APPENDIX C}

\section{BOREHOLE LOG}

\section{ENTRY BOREHOLE C4997}

C-i 
WMP-32119, Rev. 0
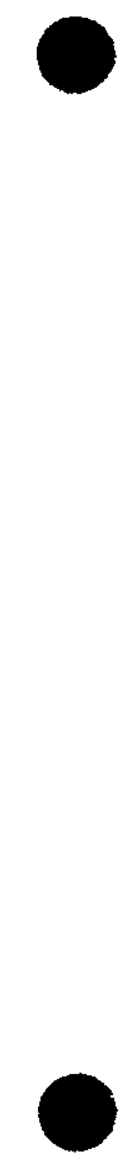

C-ii 
WMP-32119, Rev. 0

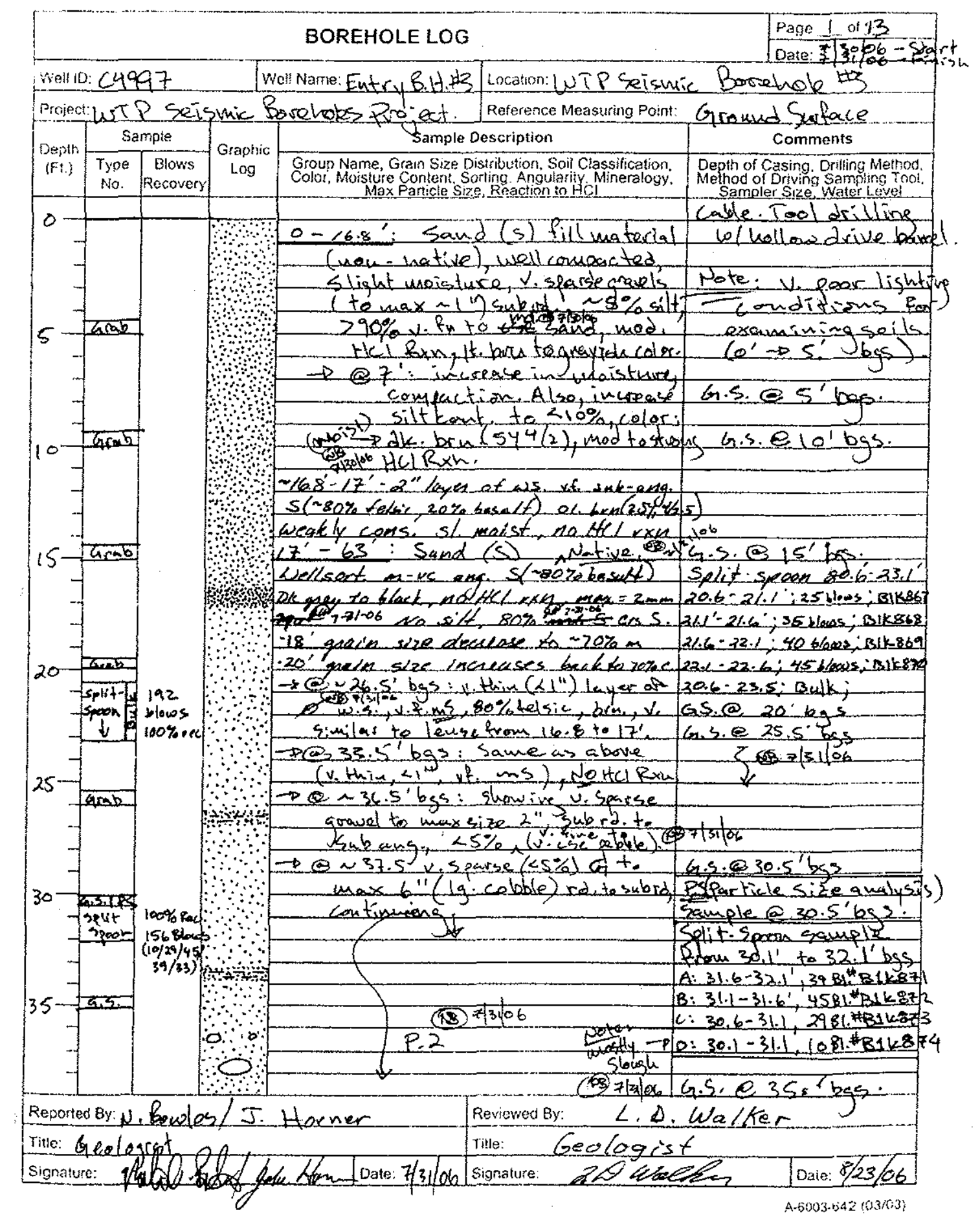

C-1 
WMP-32119, Rev. 0

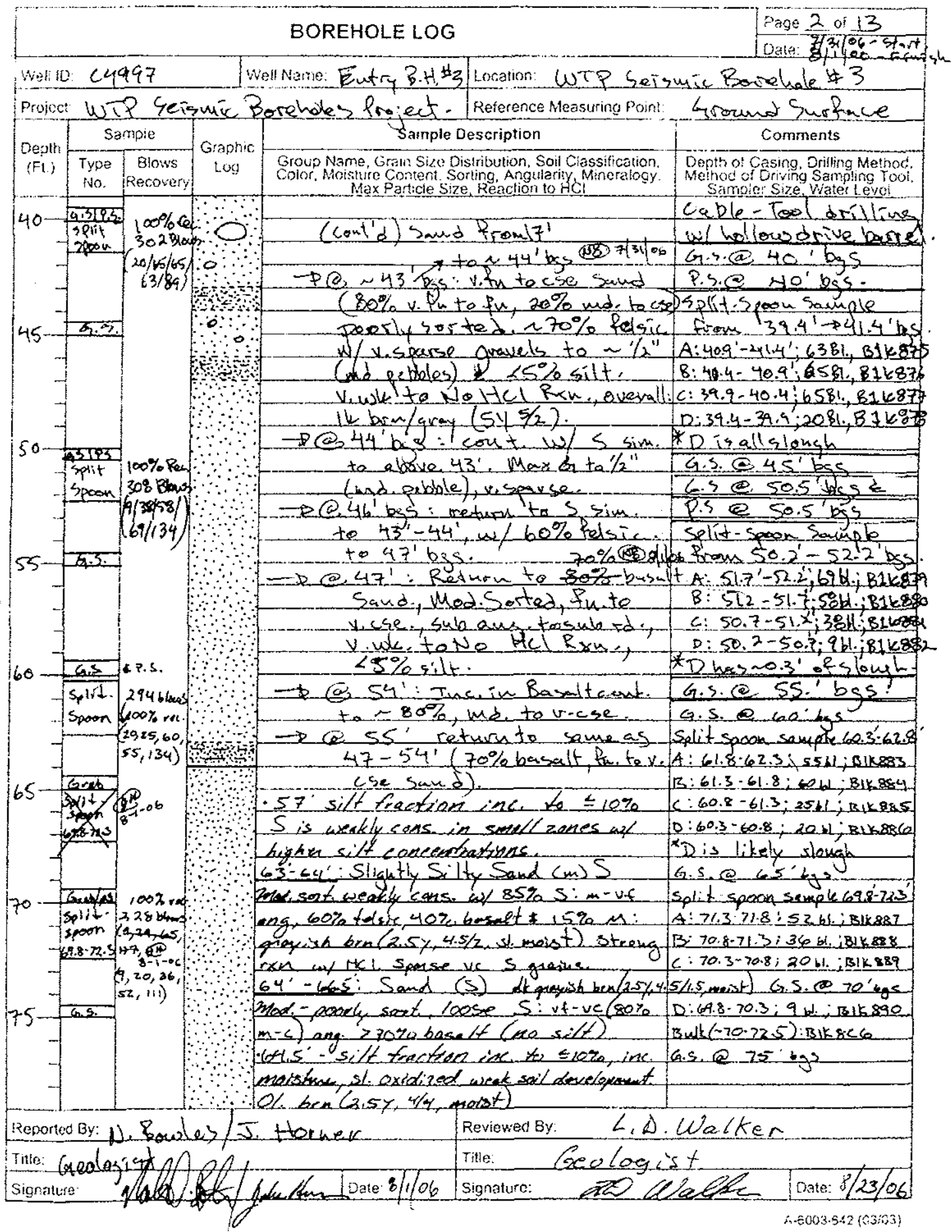

C-2 
WMP-32119, Rev. 0

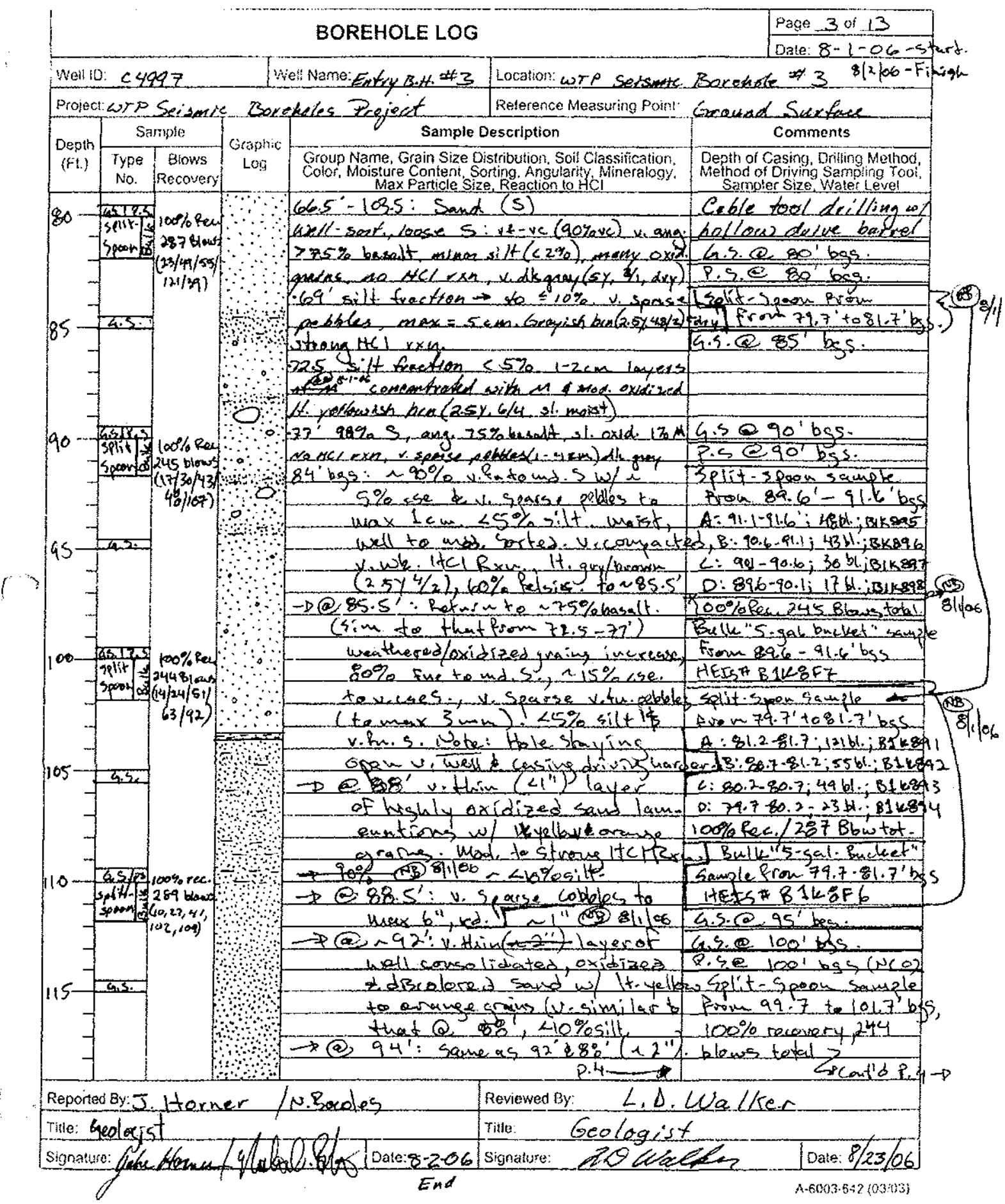


WMP-32119, Rev. 0

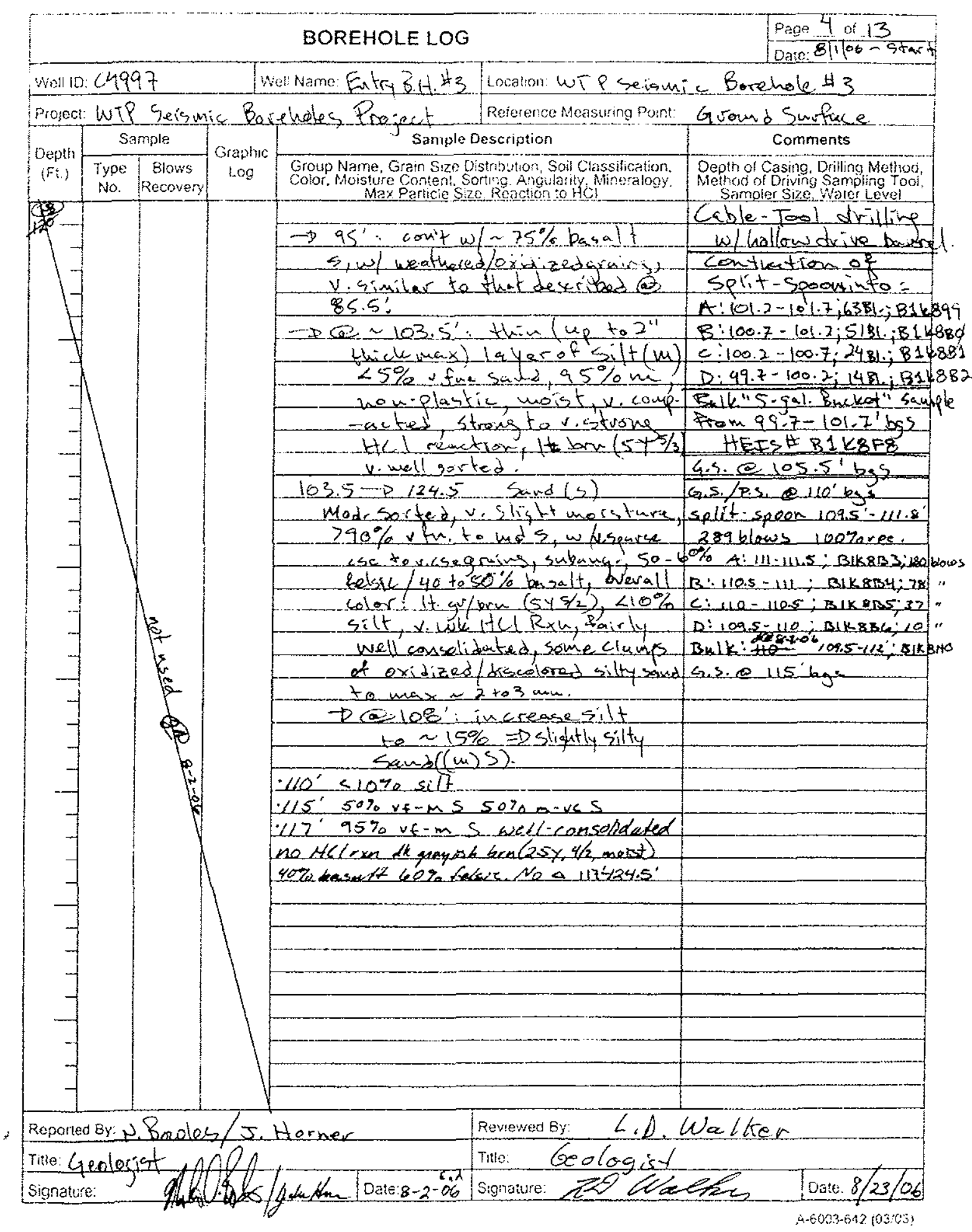


WMP-32119, Rev. 0

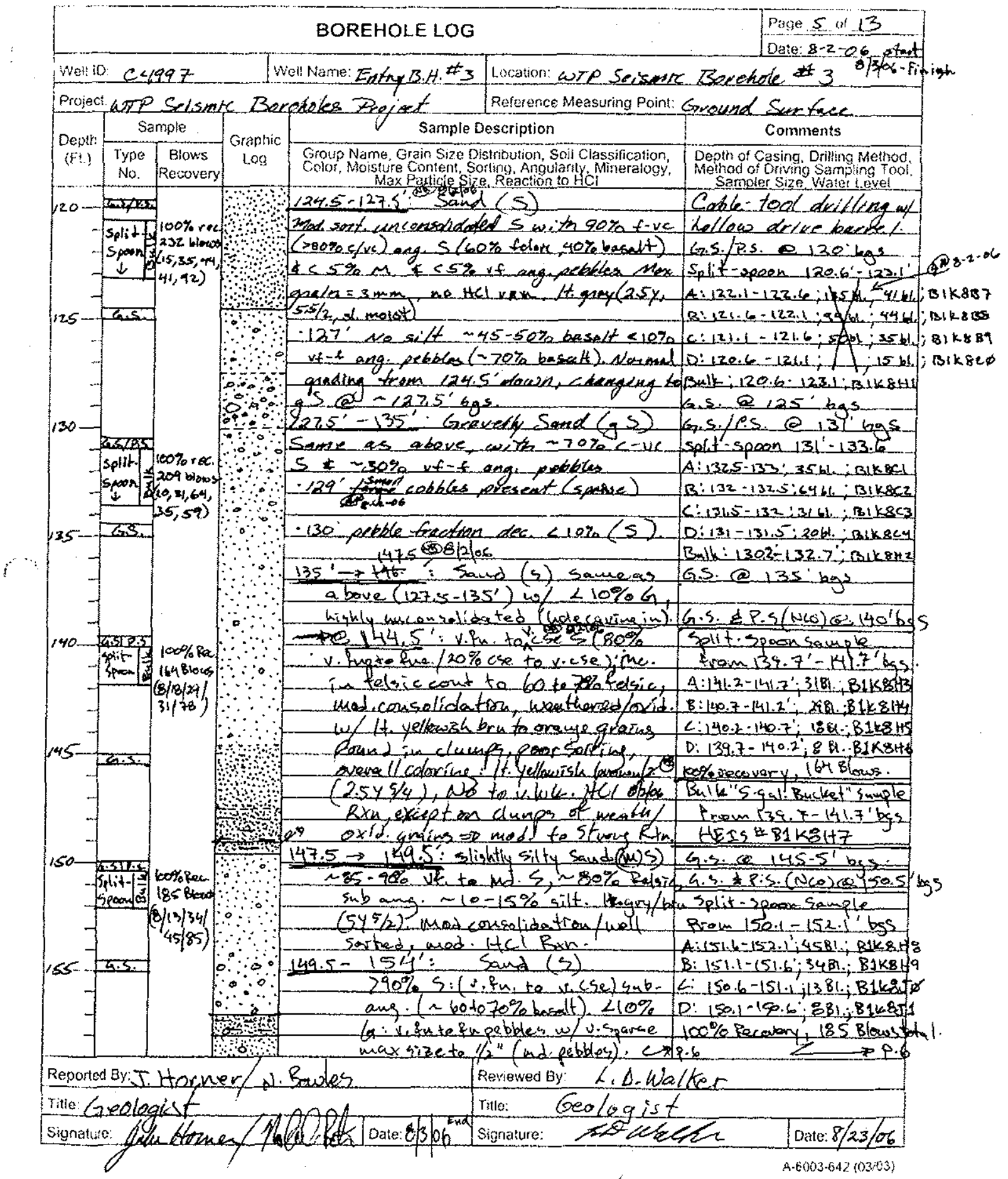


WMP-32119, Rev. 0

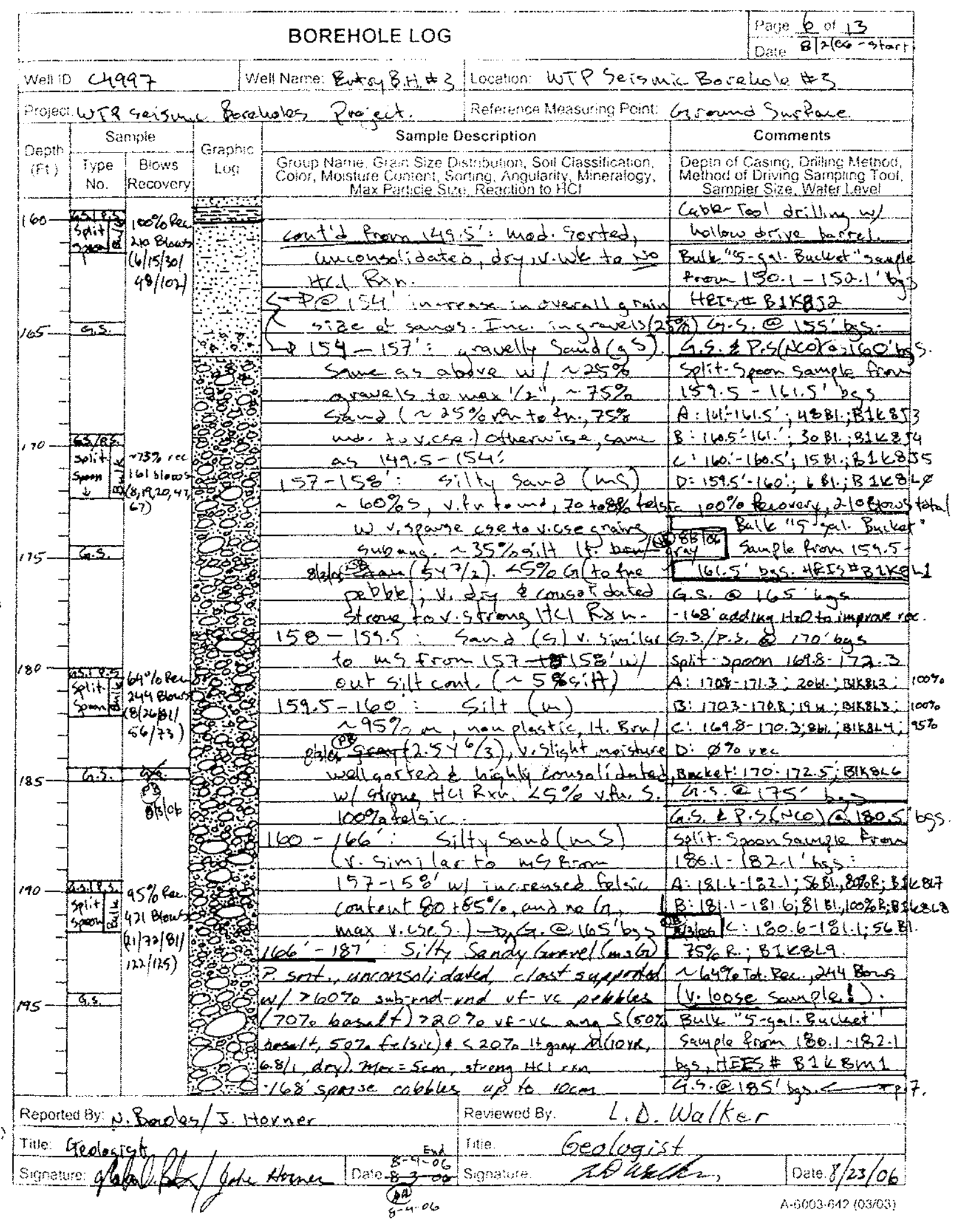


WMP-32119, Rev. 0

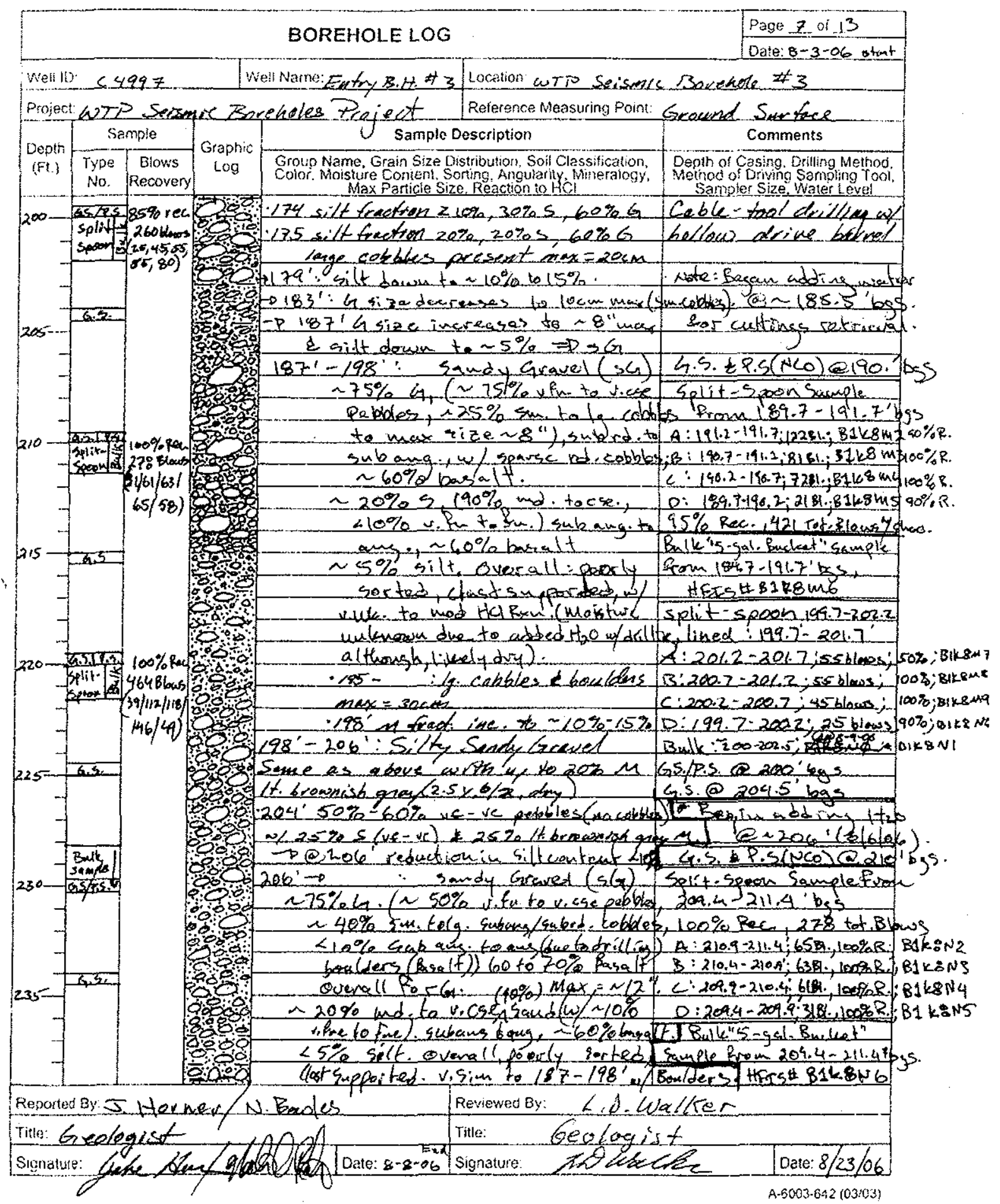


WMP-32119, Rev. 0

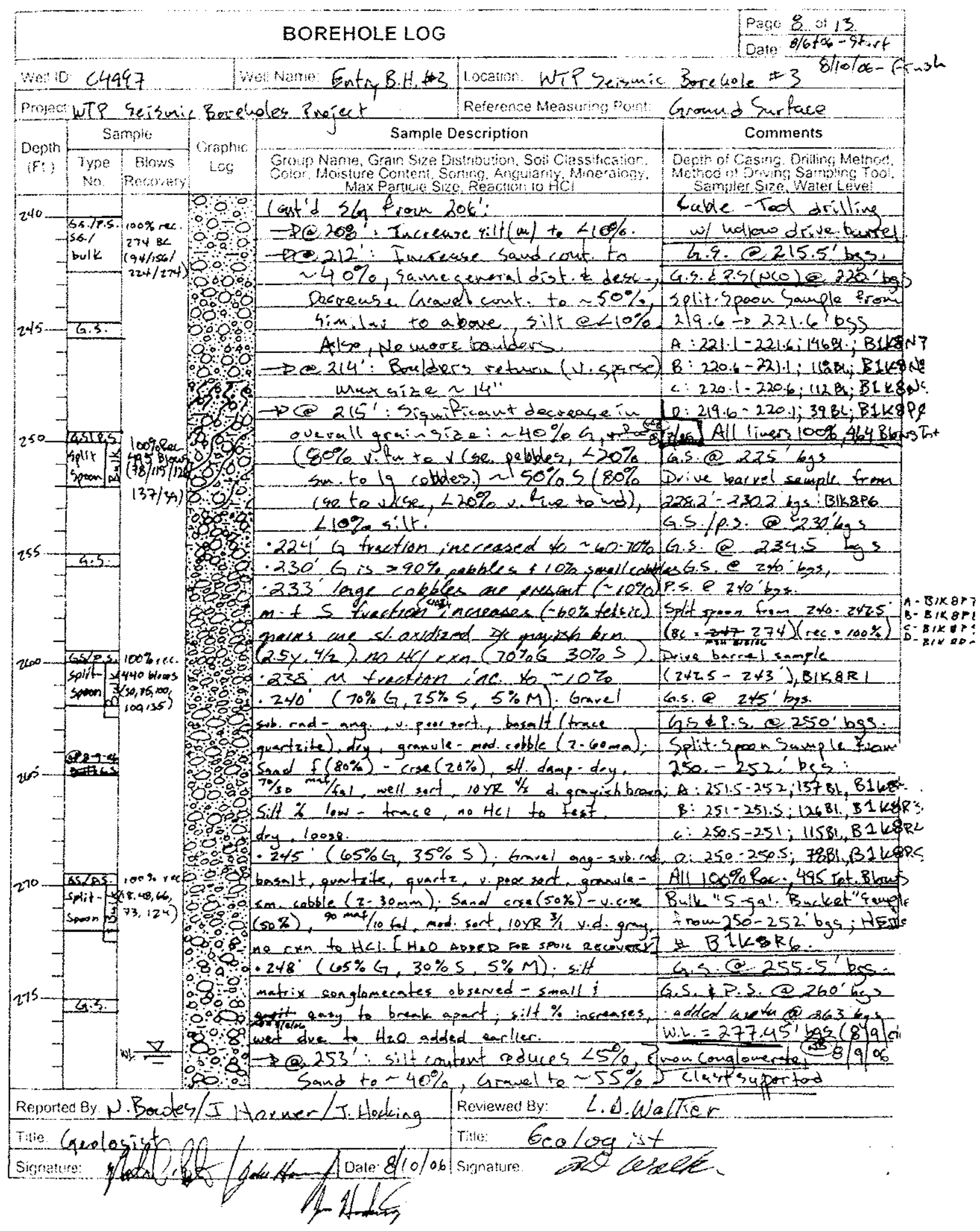


WMP-32119, Rev. 0

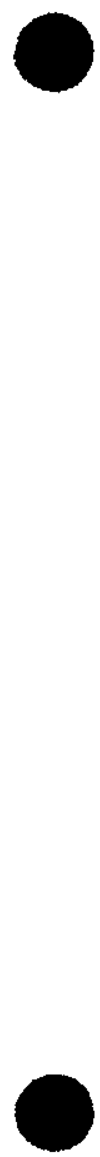

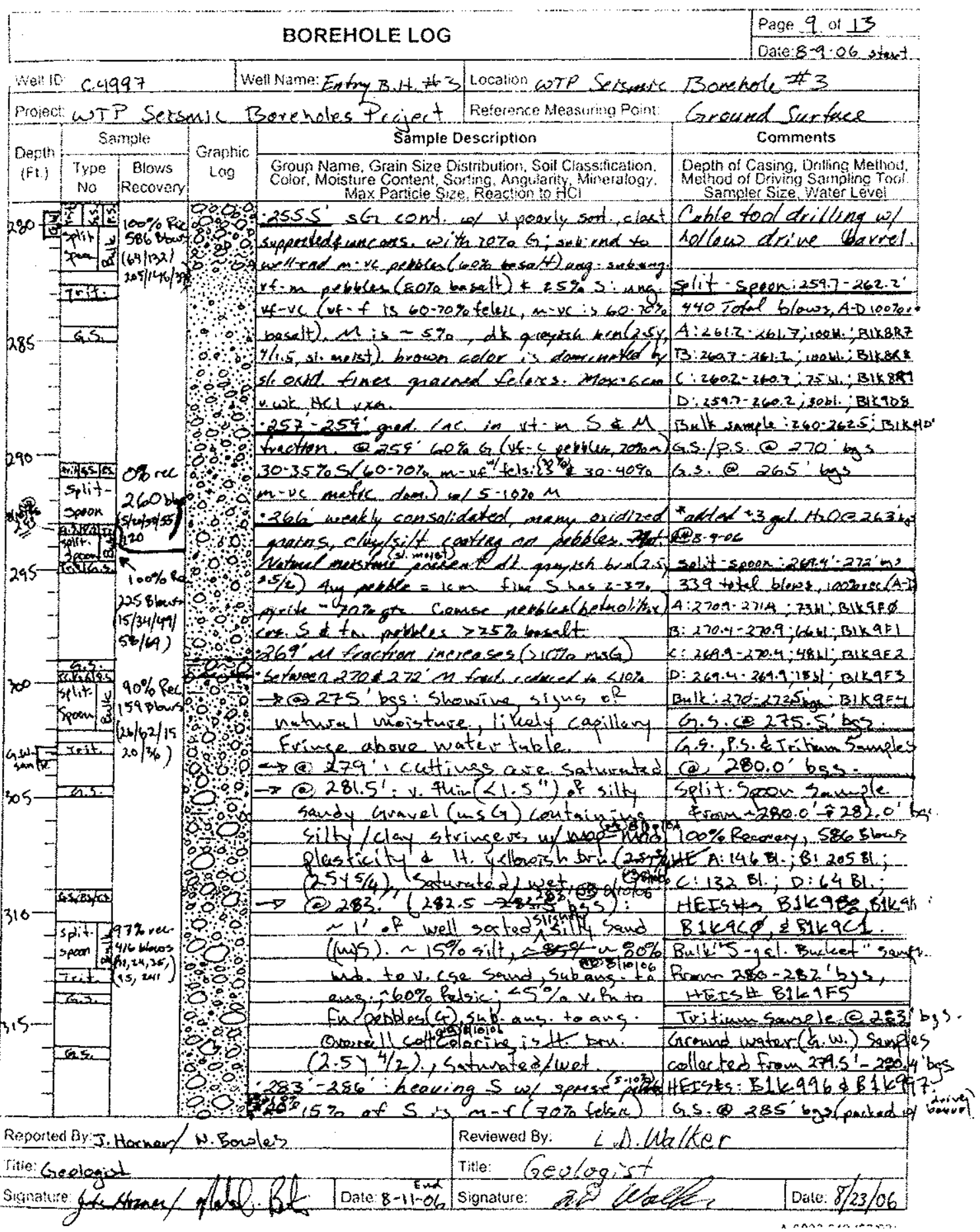


WMP-32119, Rev. 0

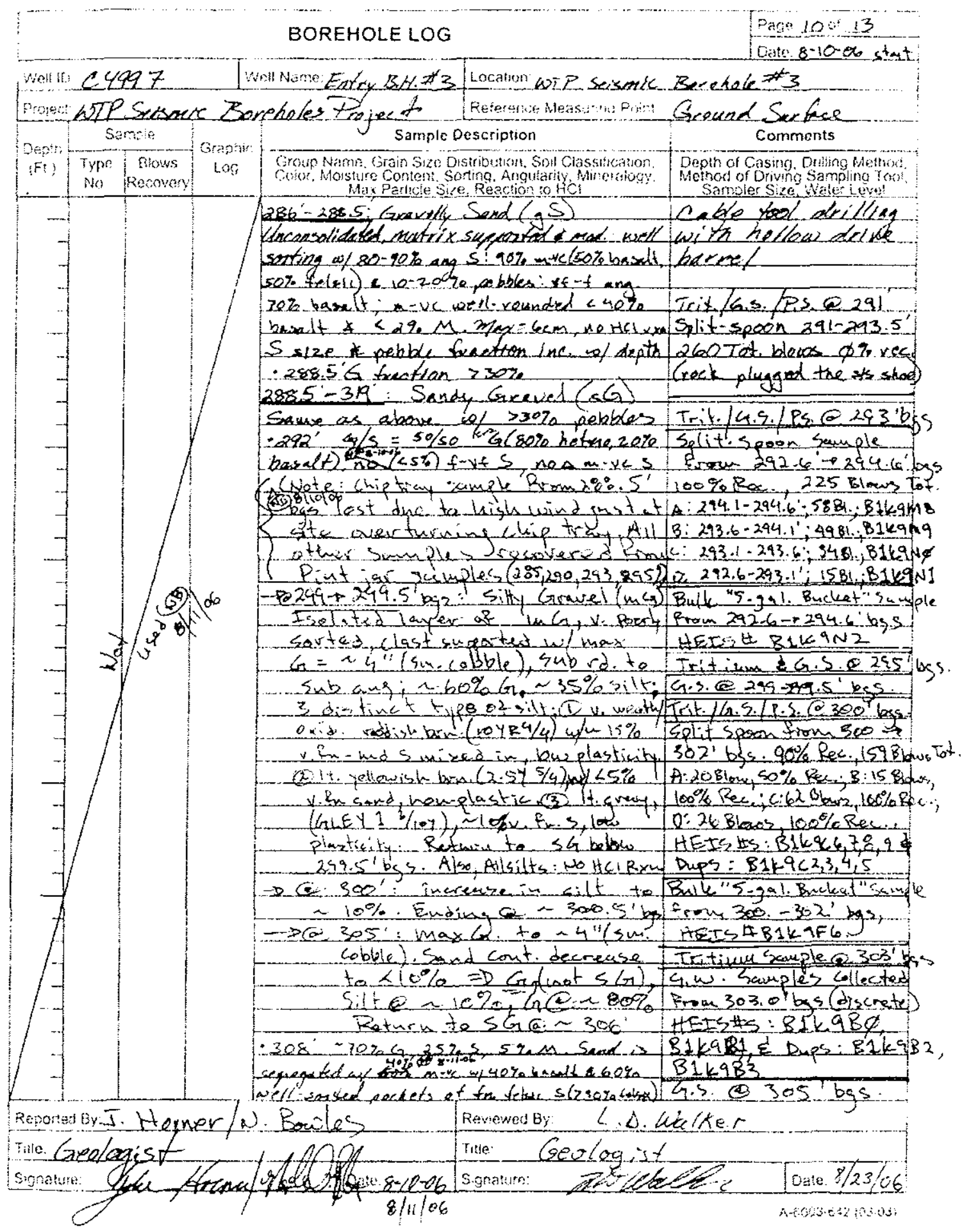


WMP-32119, Rev. 0

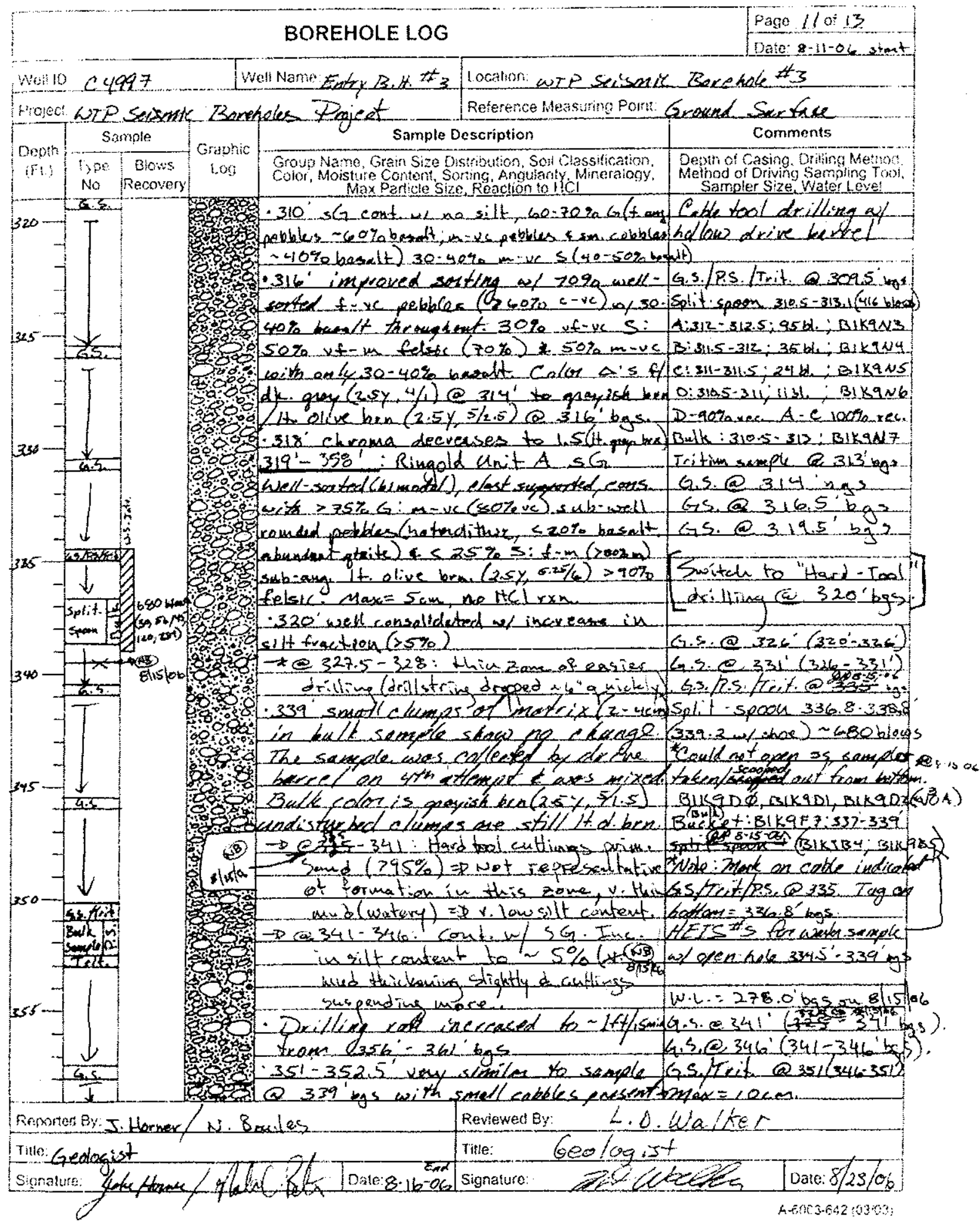


WMP-32119, Rev. 0

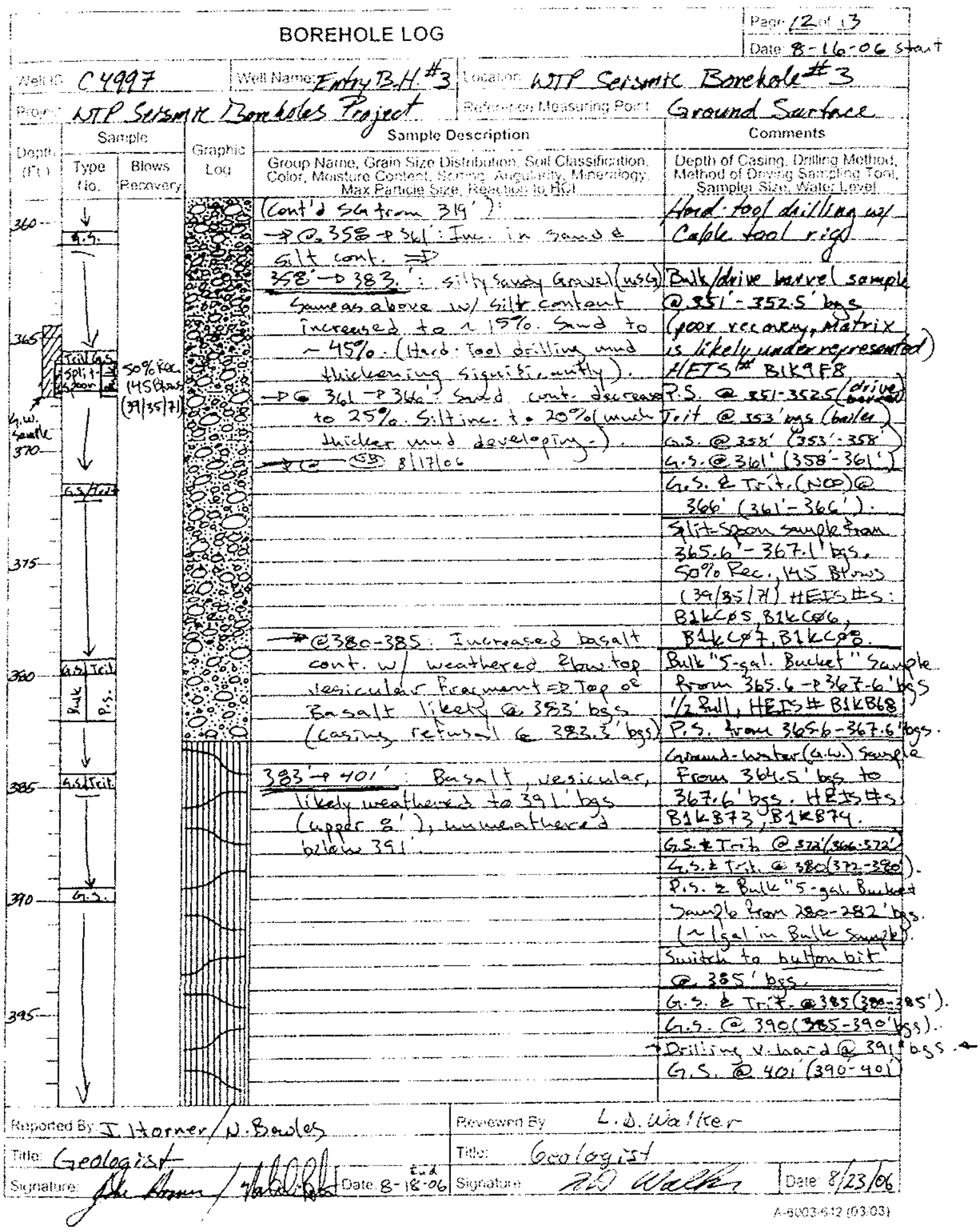


WMP-32119, Rev. 0

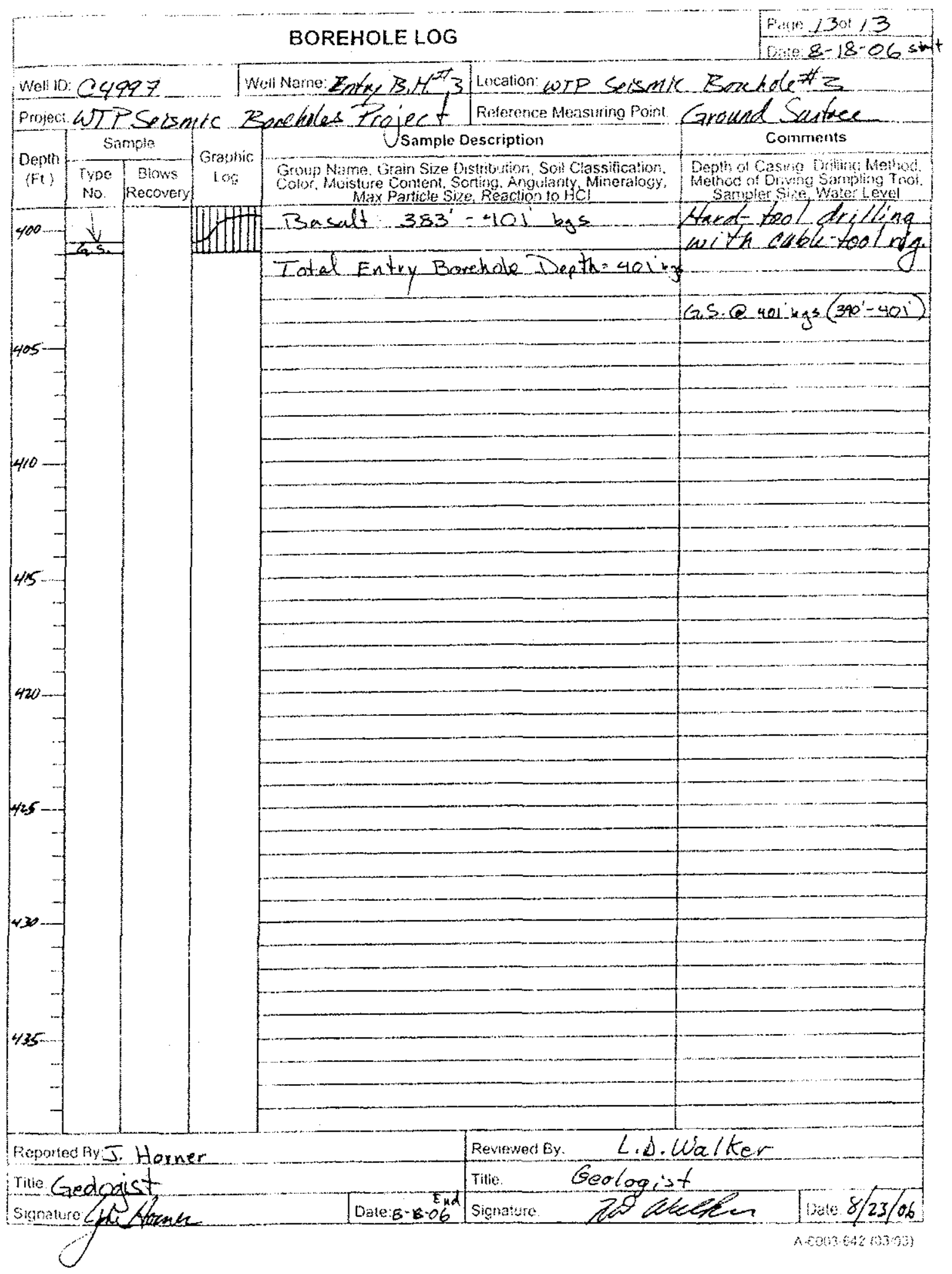

C-13 
WMP-32119, Rev. 0

This page intentionally left blank 
WMP-32119, Rev. 0

\section{APPENDIX D}

BOREHOLE LOG

ENTRY BOREHOLE C4998

D-i 
WMP-32119, Rev. 0

D-ii 
WMP-32119, Rev. 0

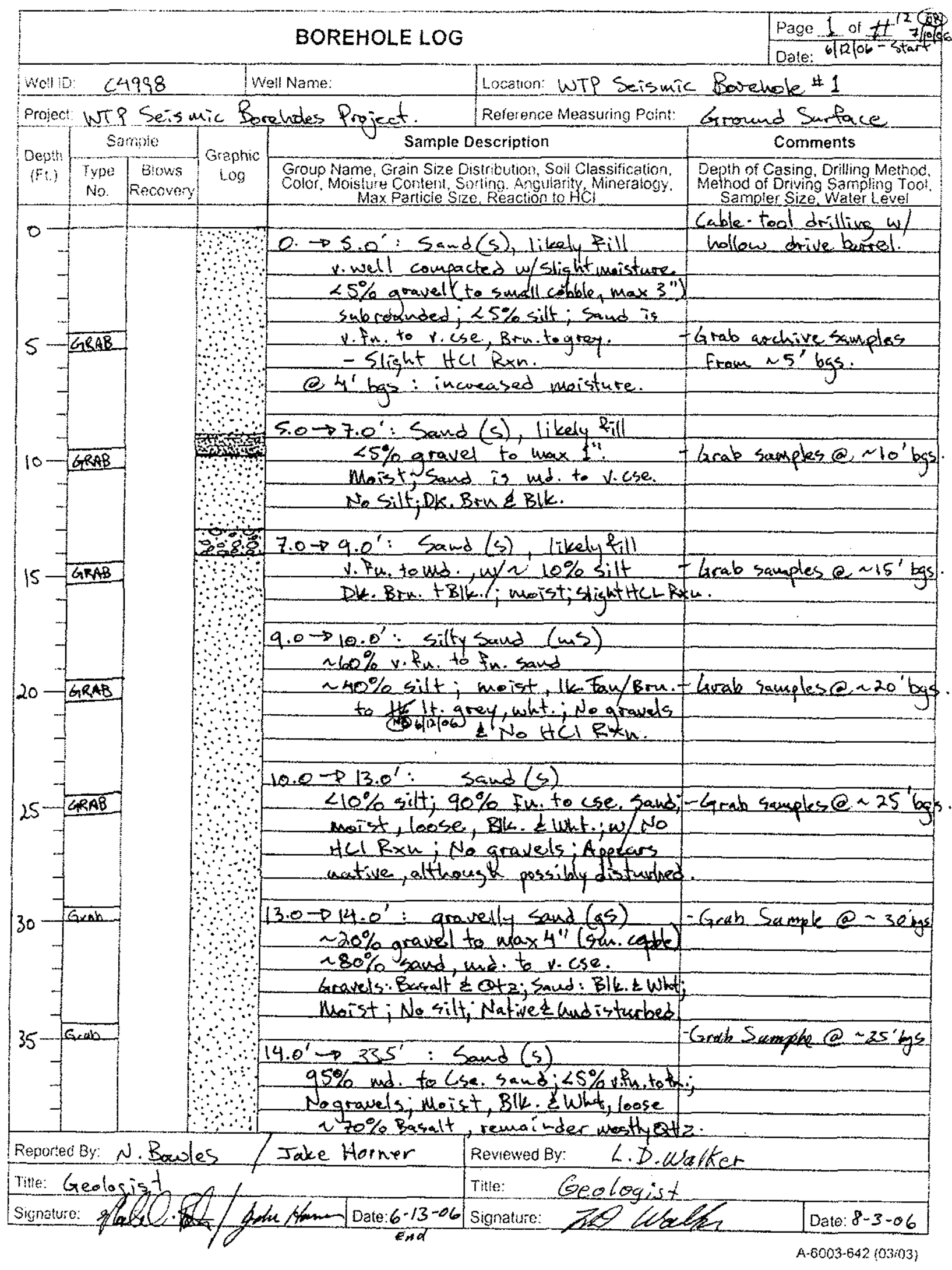


WMP-32119, Rev. 0

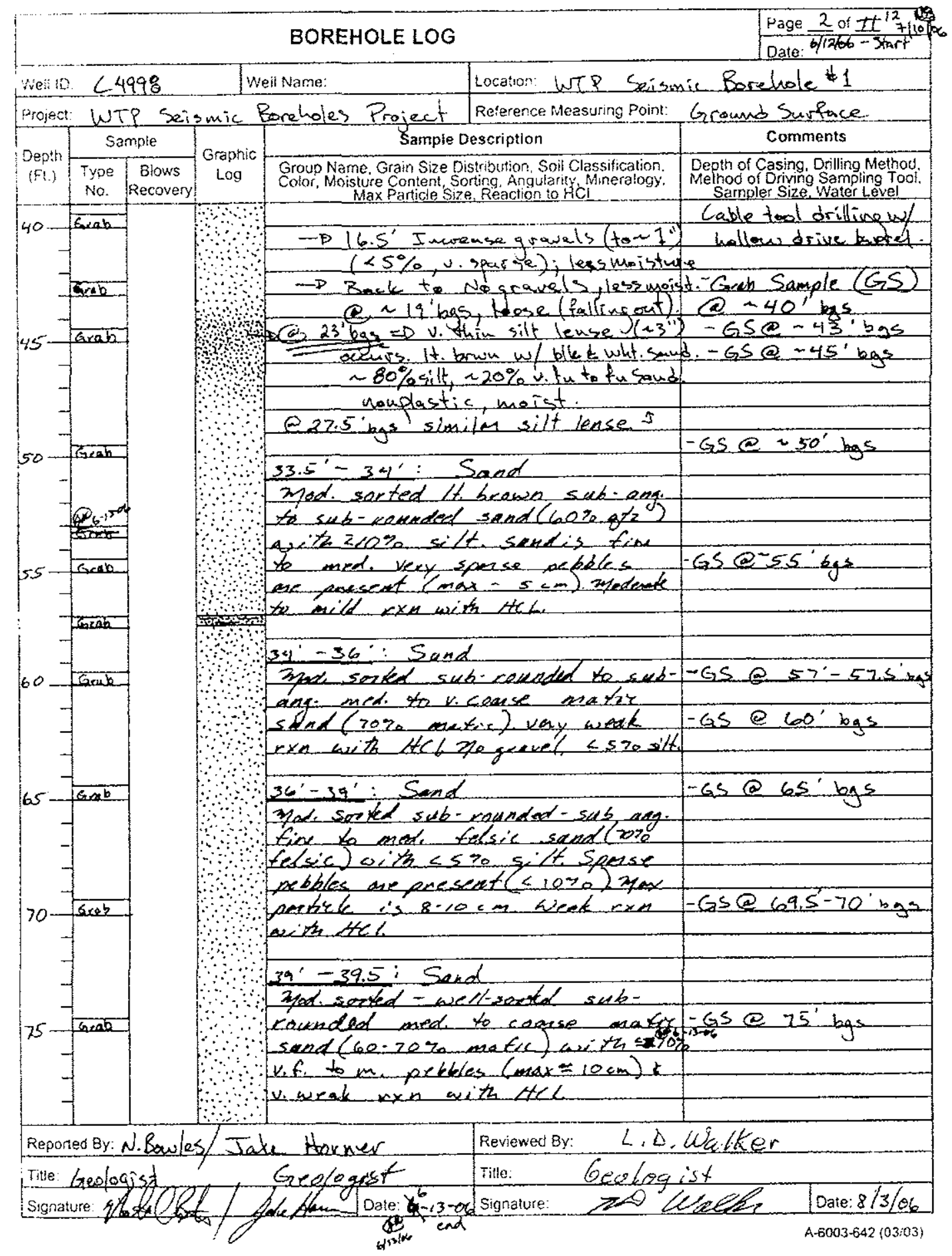

D-2 
WMP-32119, Rev. 0

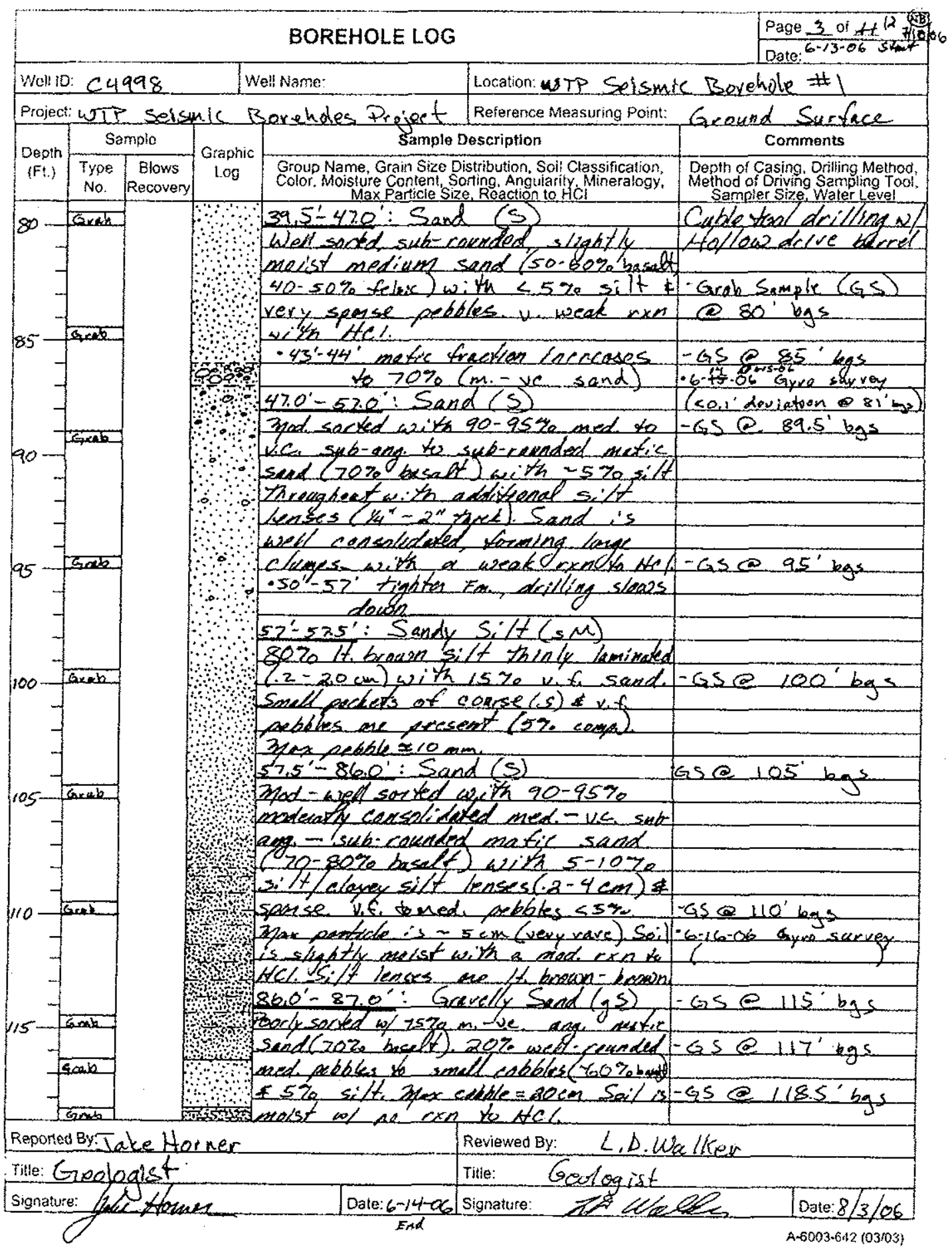


WMP-32119, Rev. 0

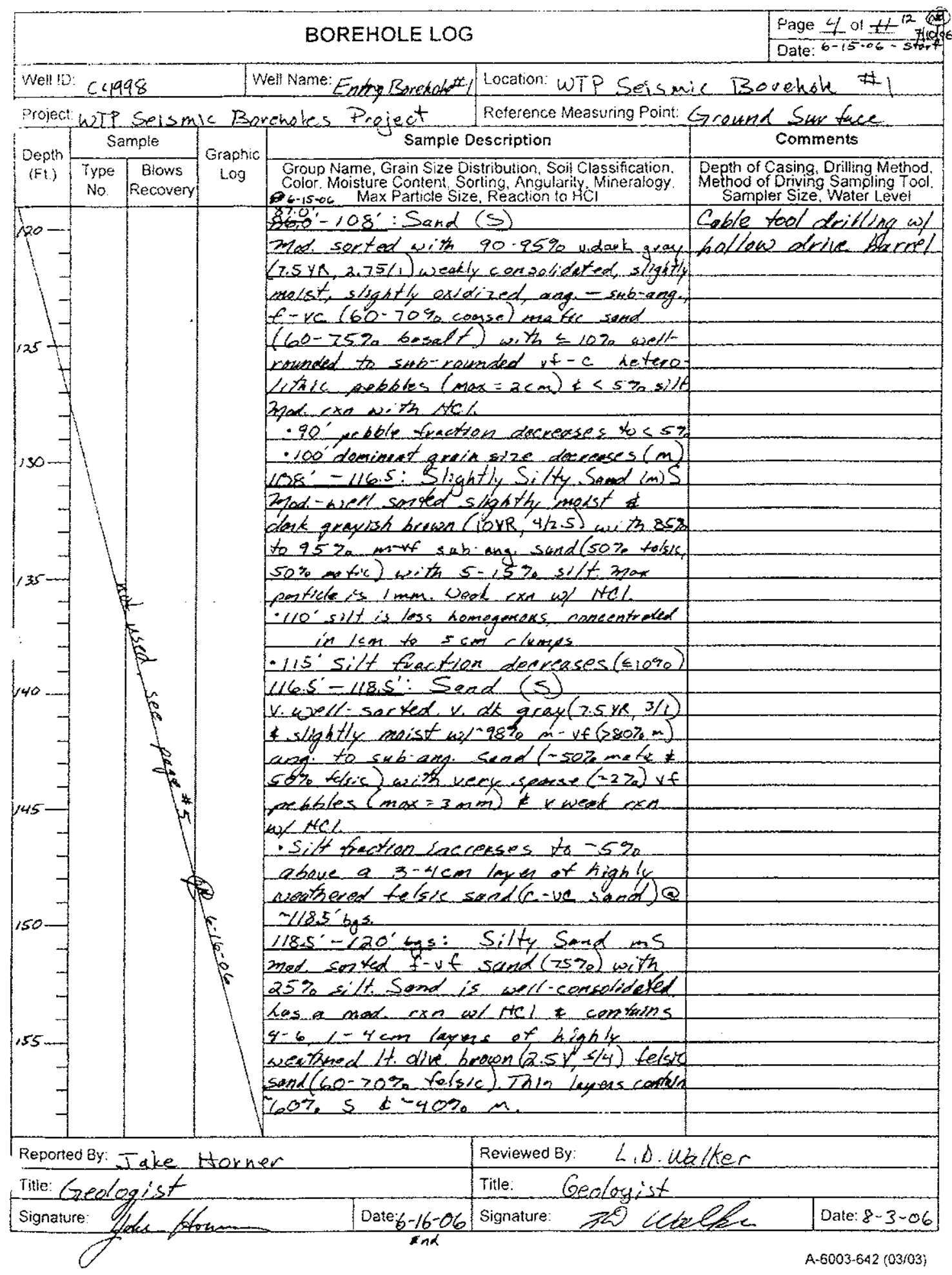


WMP-32119, Rev. 0

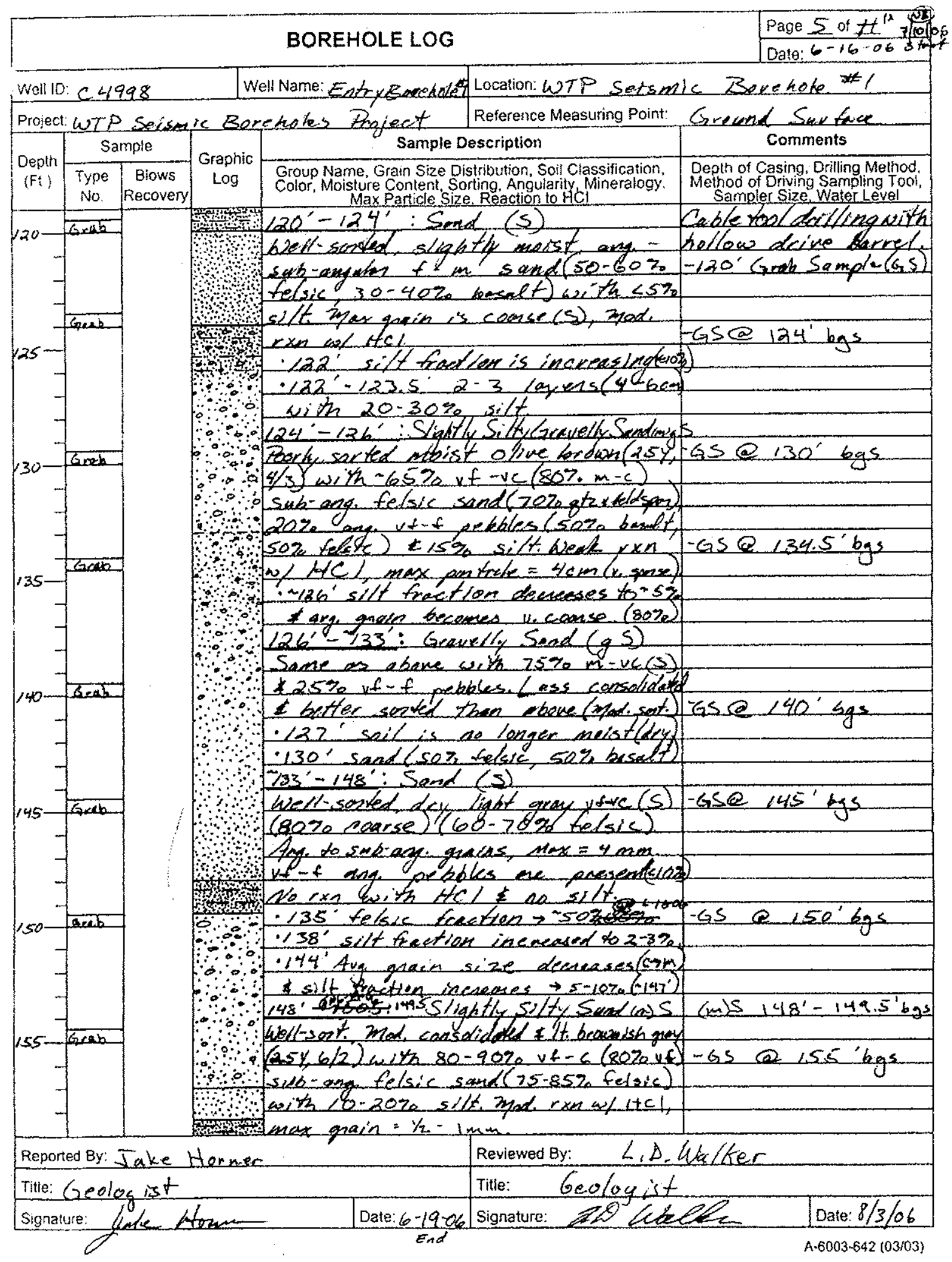




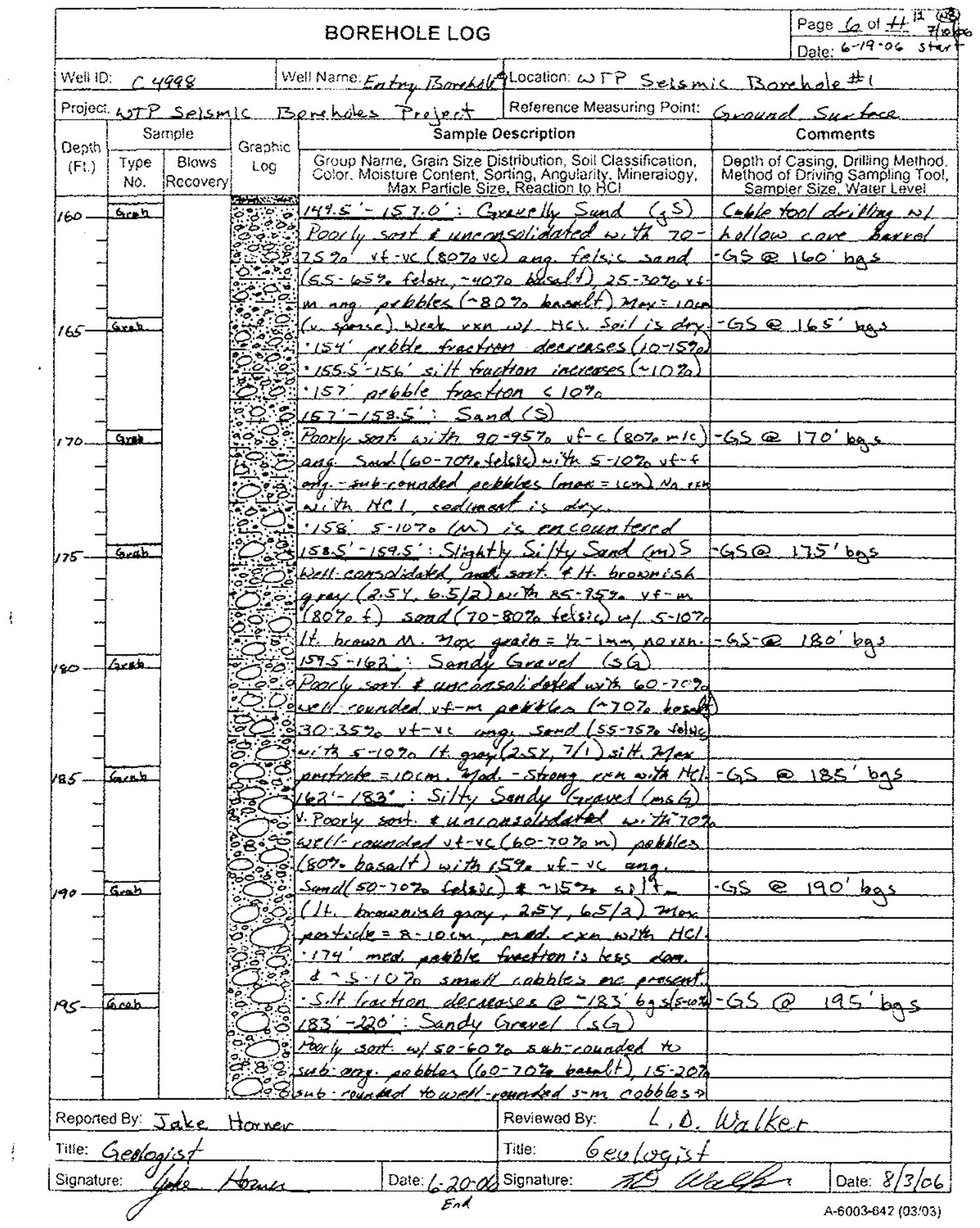


WMP-32119, Rev. 0

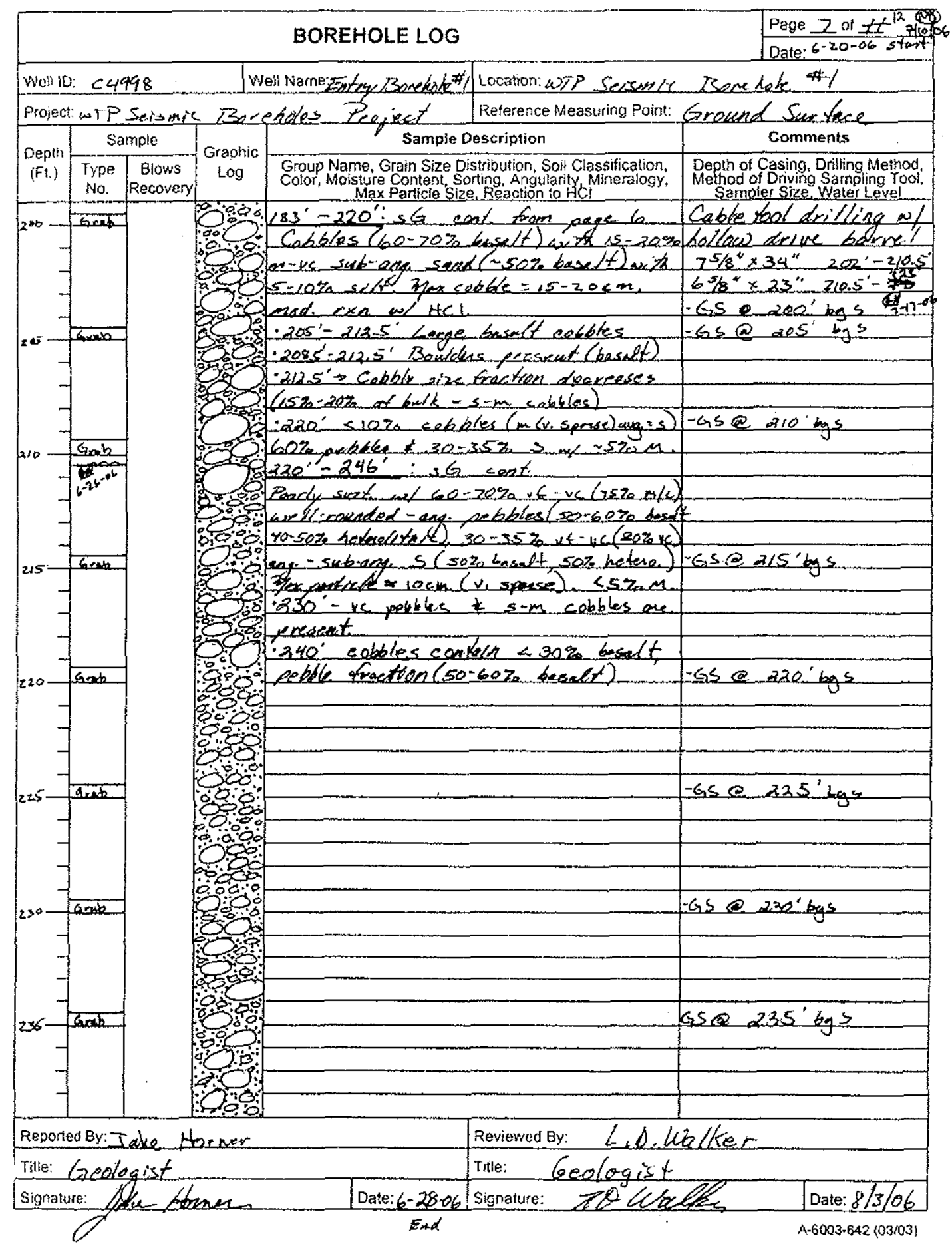




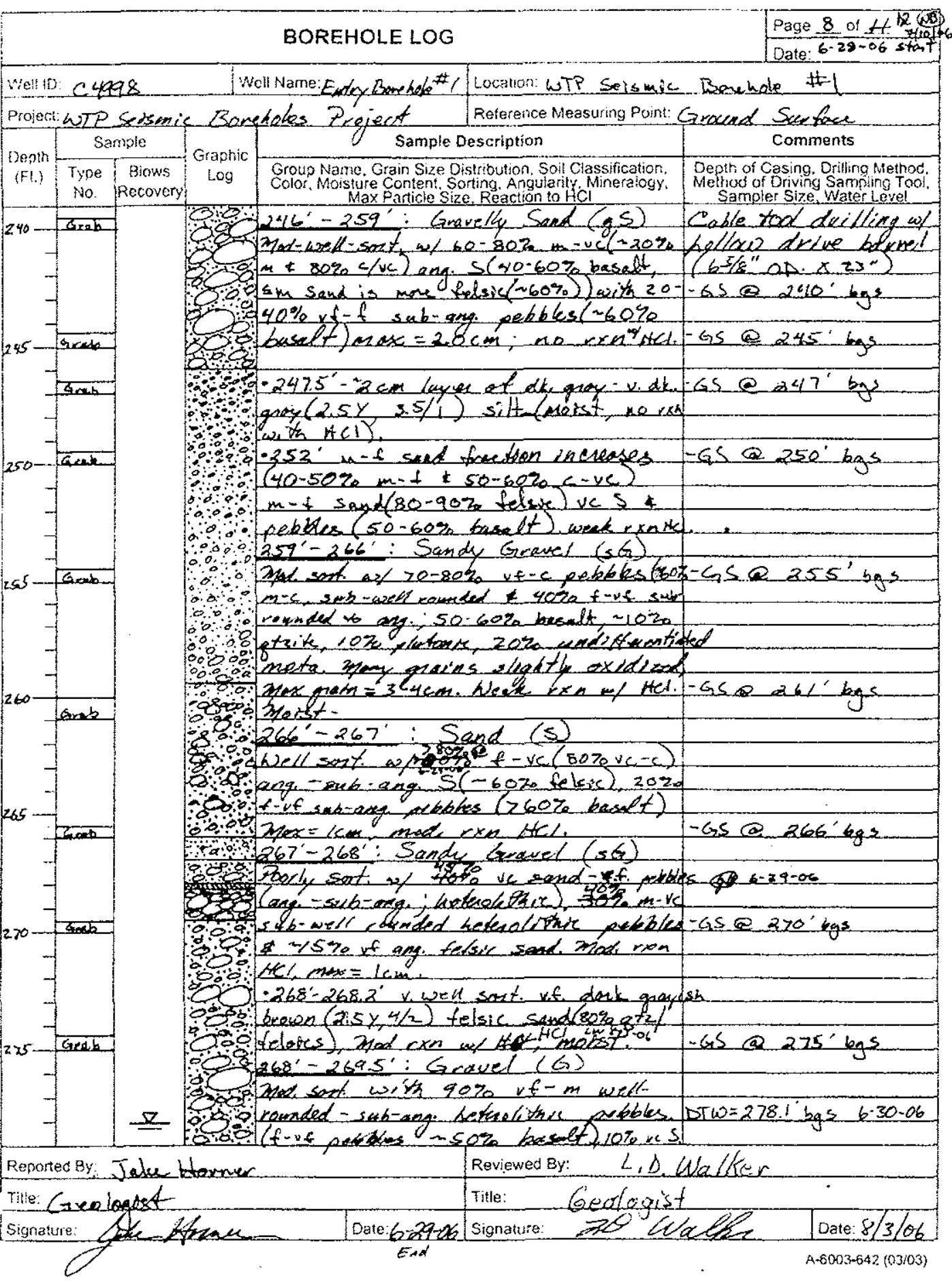


WMP-32119, Rev. 0

BOREHOLE LOG

\begin{tabular}{|c|c|c|}
\hline Well ID: c sp98 & Well Name:Estry Borehole*1 & Location: WTP Seismie Bonehole*1 \\
\hline
\end{tabular}

Project: wrP Soismic Boreholes Preject Reference Measuring Point: Ground Sur toe \begin{tabular}{|c|c|c|c|c|c|}
\hline Sample & Gaphic & Comple Description & Coments
\end{tabular}

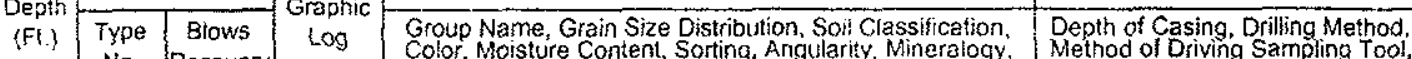

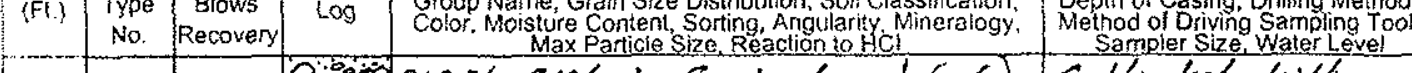

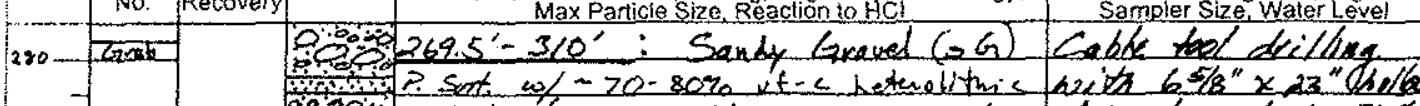

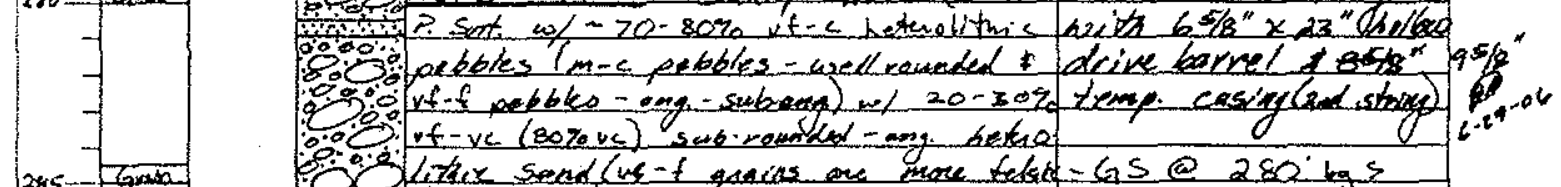

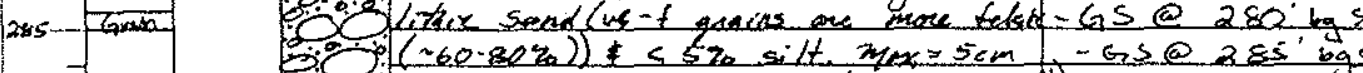

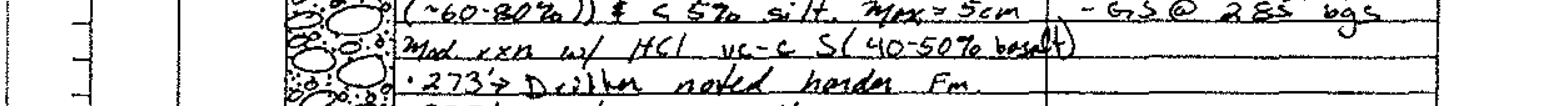

$7 \quad 273^{\prime} \rightarrow 274^{\prime} 5 \cdot m$ cokbes, maks $=15 \mathrm{~cm}$

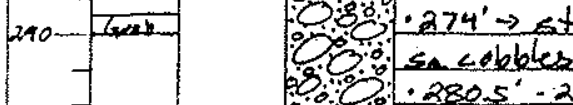

-280.5 -281'ugs Sand $(3)$

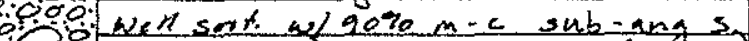

c. $(60 \%$ felste $)$ it. dive broion $(2.5 \% 5 \%$

$295-6$ arab

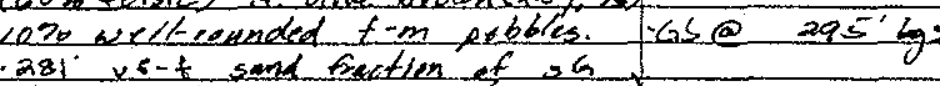

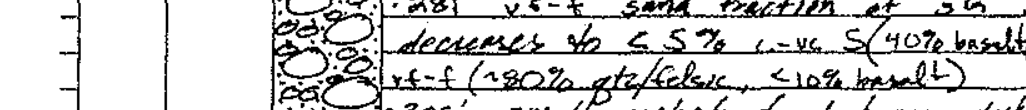

(rand

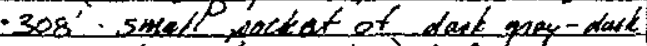

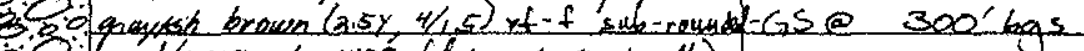

send (t80\%, gts, $10 \%$ feletes, $40 \%$ baseft)

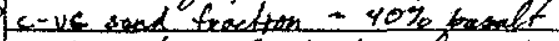

310'-322': Sandy breved cout....

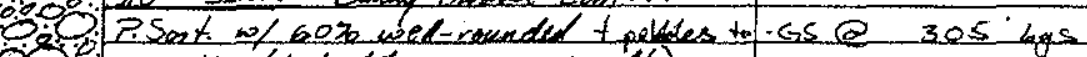

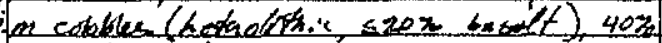

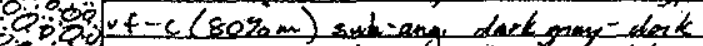

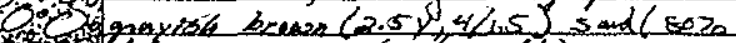

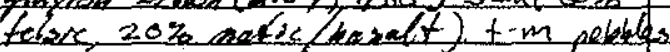

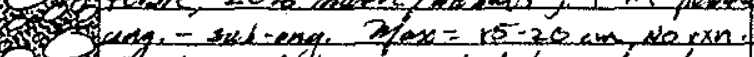

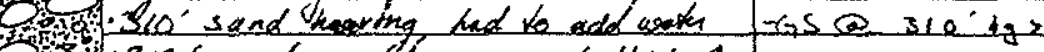

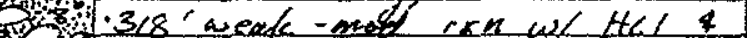

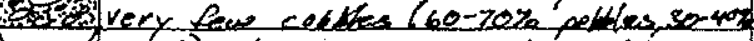

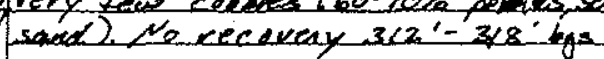

Reported By: Jake. Hor user

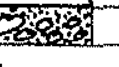

$\operatorname{cas} 2318^{\prime}$ tgs

Titie: Geologist

Signature: folke fremer 7

\begin{tabular}{l|l} 
& Titie cologist
\end{tabular} 7-5-06 Signature: $\mathbb{Z}$ tale Date: $8 / 3 / 06$

A.6003.642 (03/03) 
WMP-32119, Rev. 0

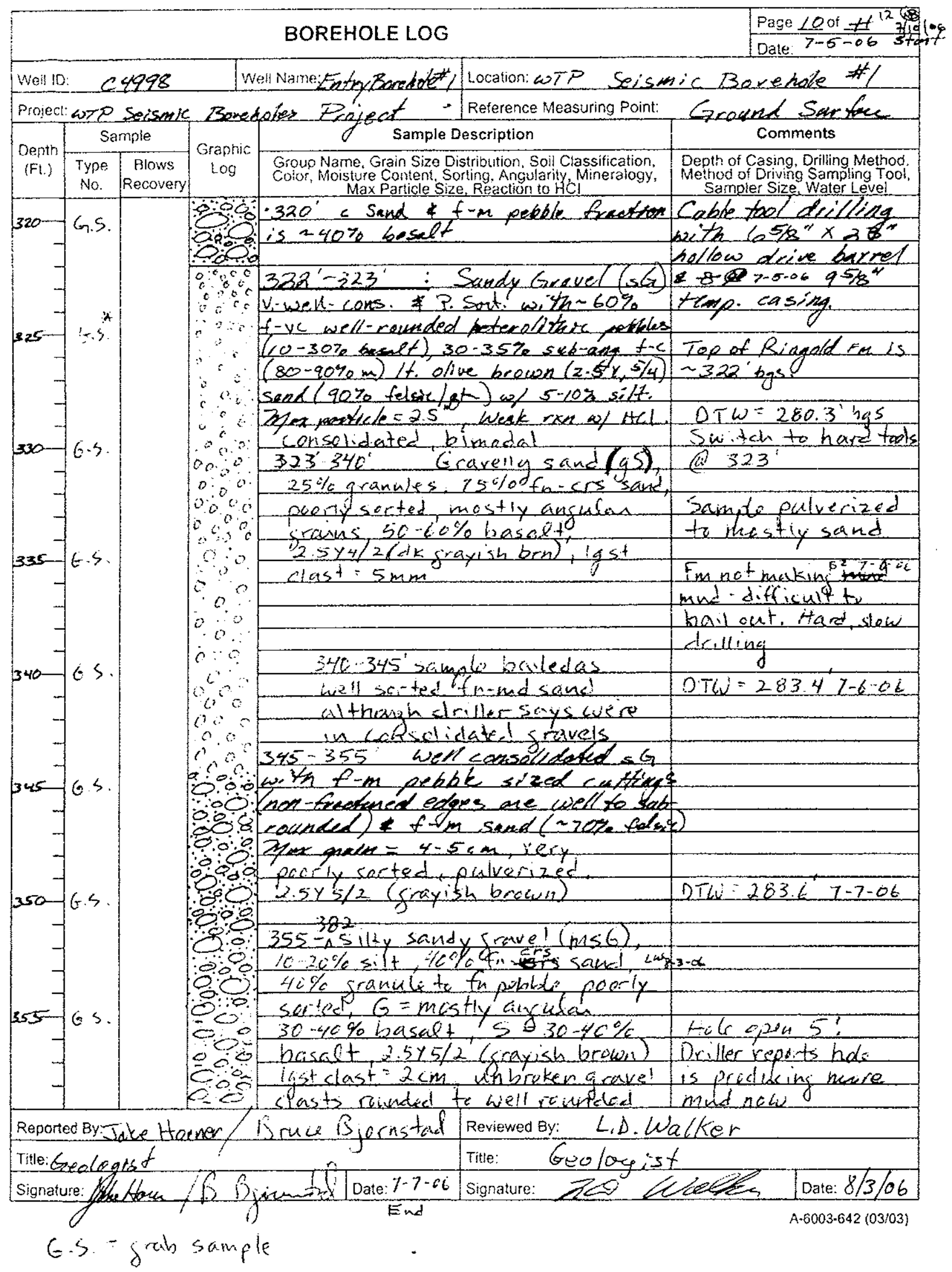


WMP-32119, Rev. 0

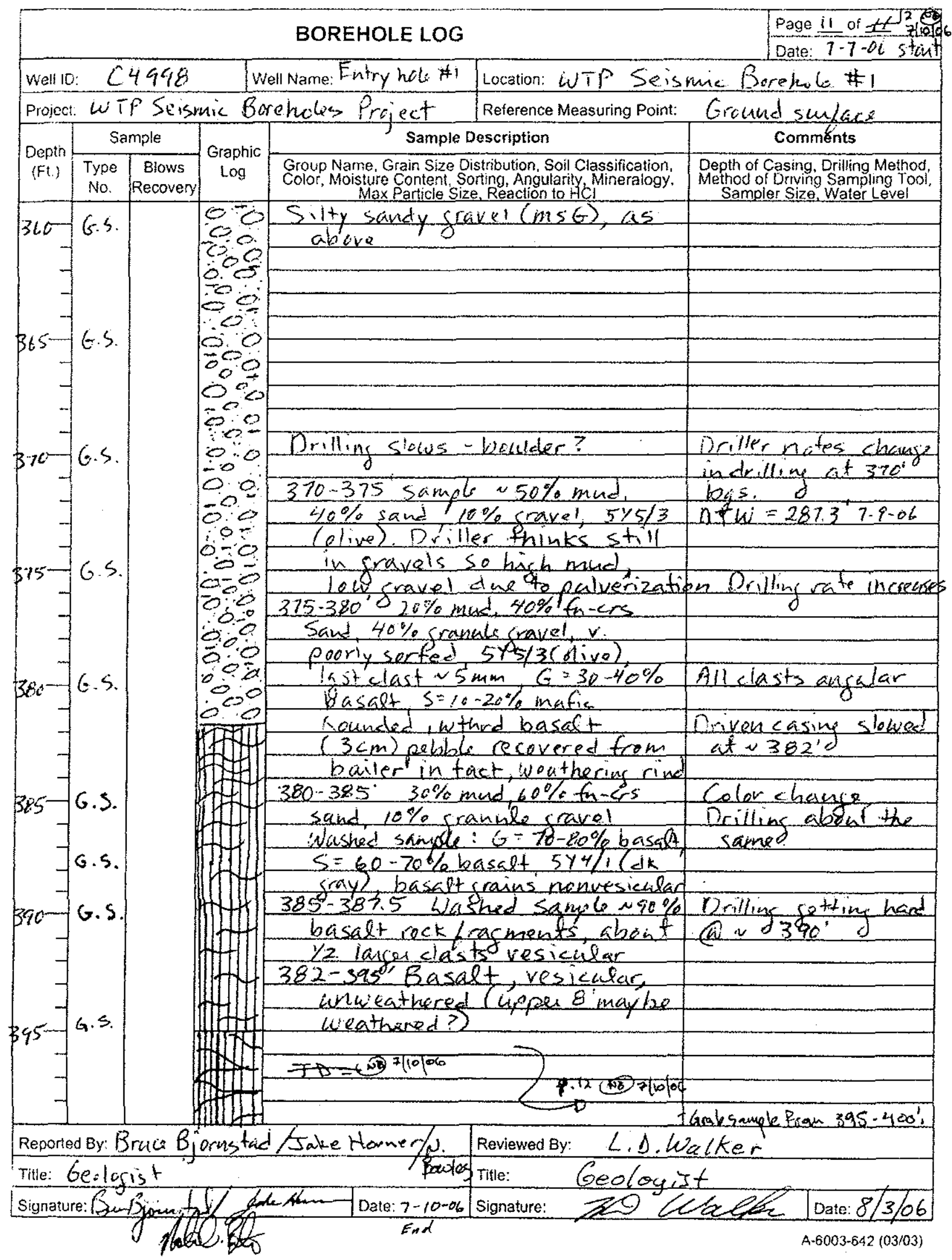


WMP-32119, Rev. 0

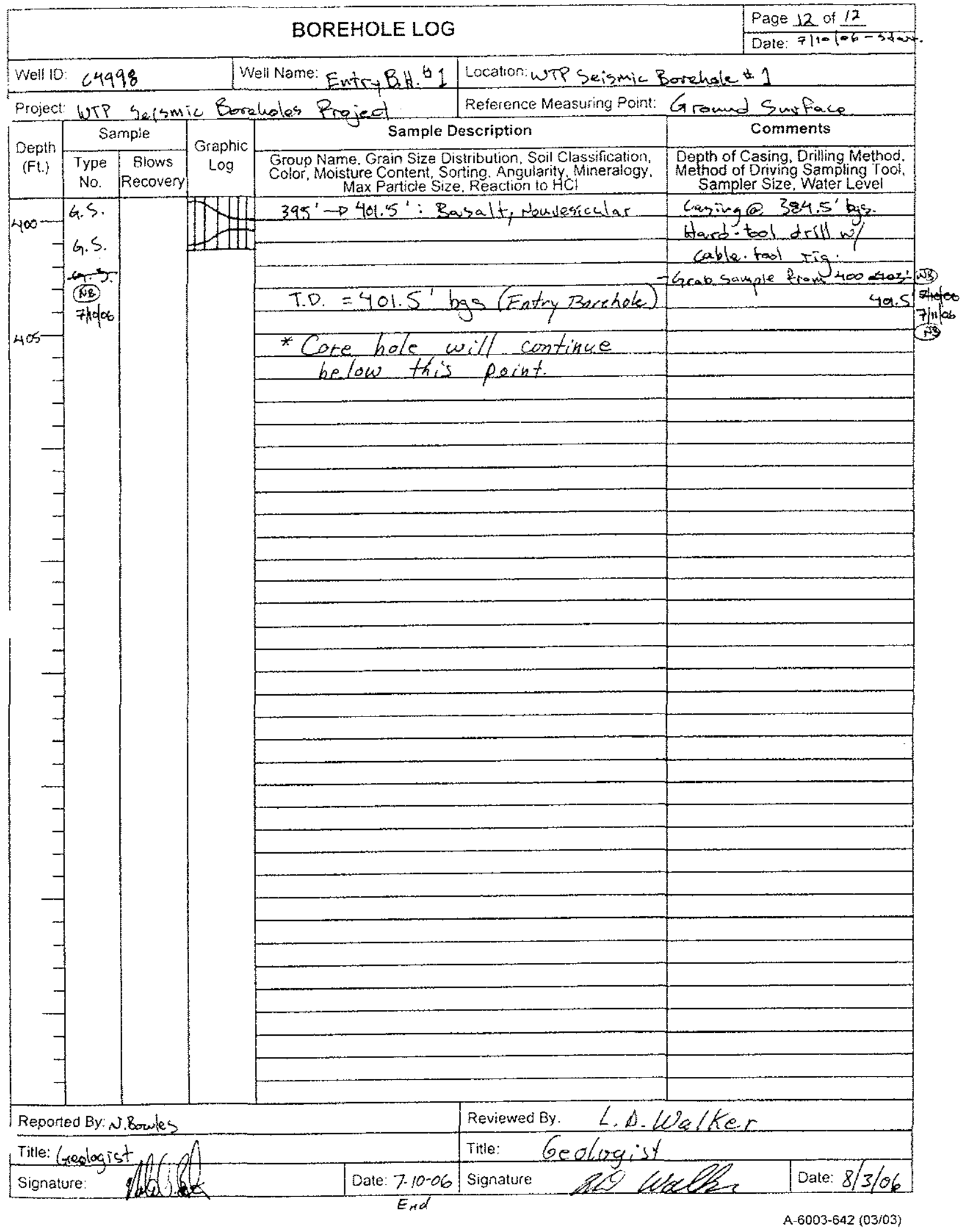

D-12 
WMP-32119, Rev. 0

\section{APPENDIX E}

\section{CHIP TRAY PHOTOGRAPHS (C4993)}

E-i 
WMP-32119, Rev. 0

This page intentionally left blank.

E-ii 
WMP-32119, Rev. 0

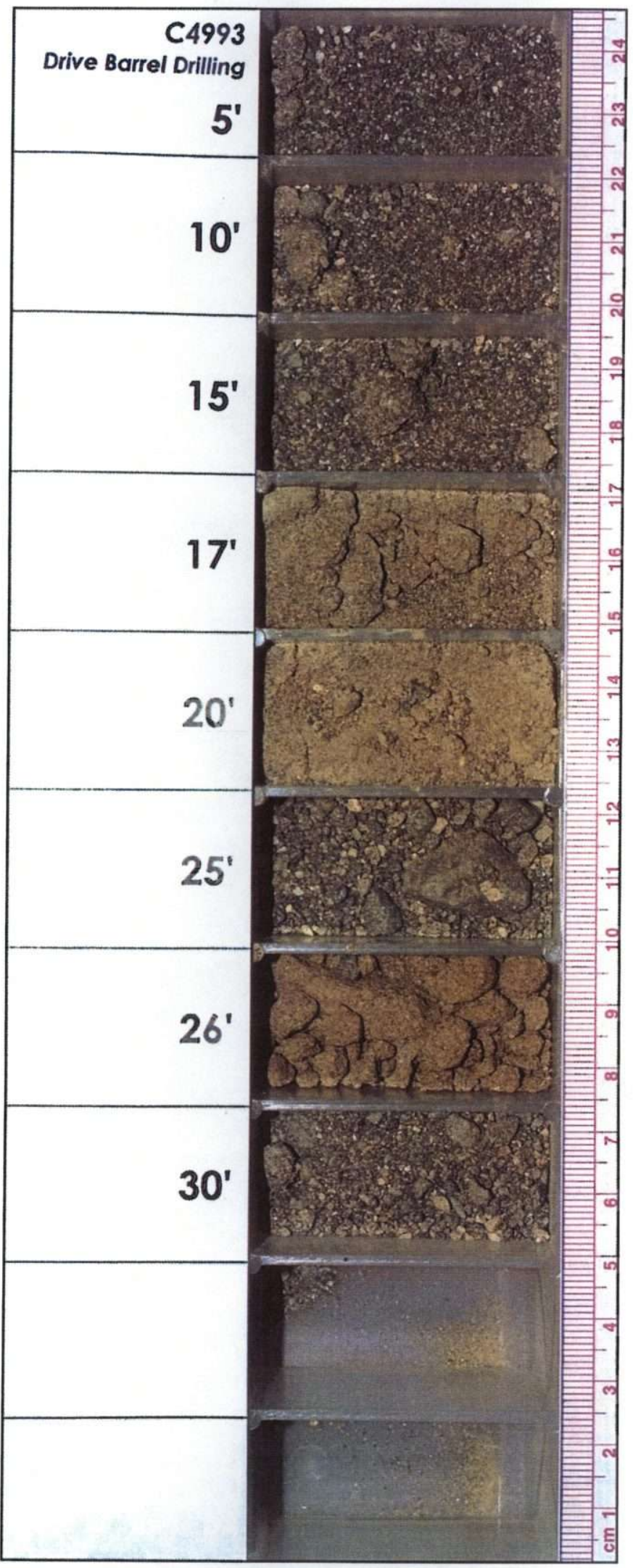

E-1 
WMP-32119, Rev. 0

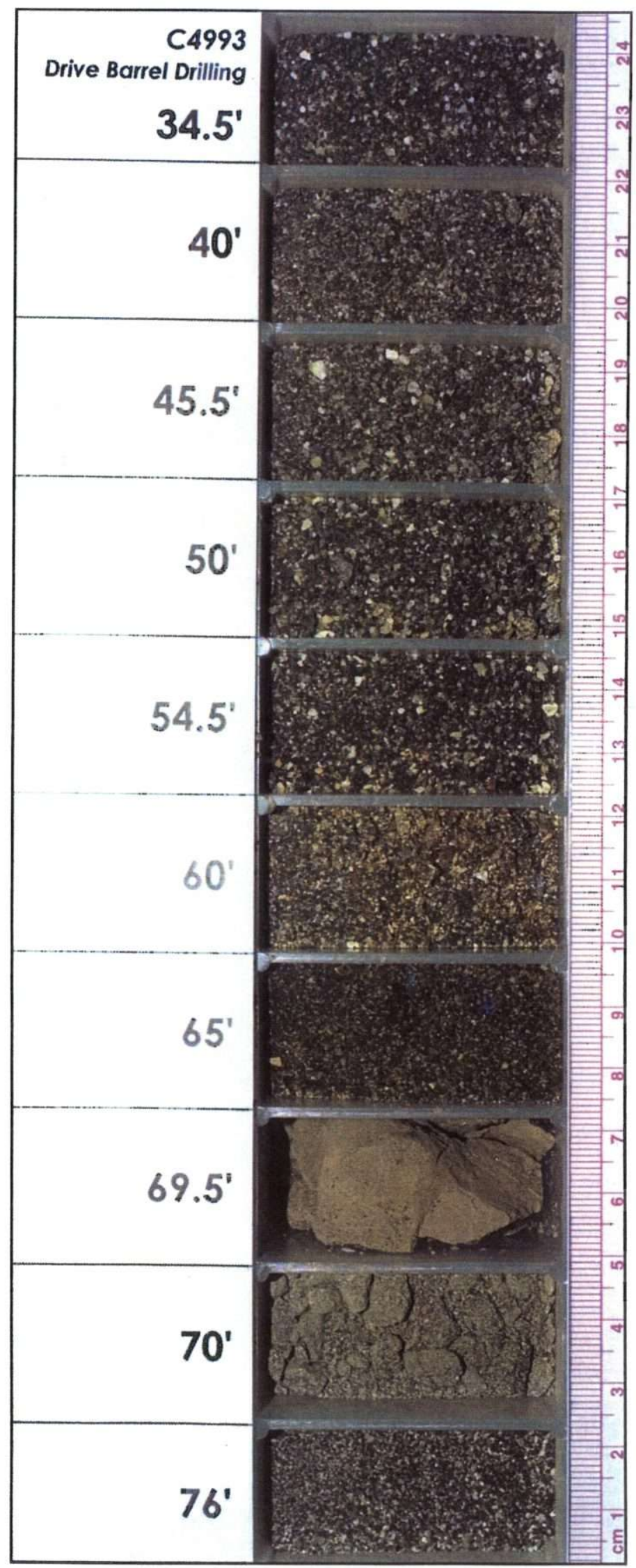

E-2 
WMP-32119, Rev. 0

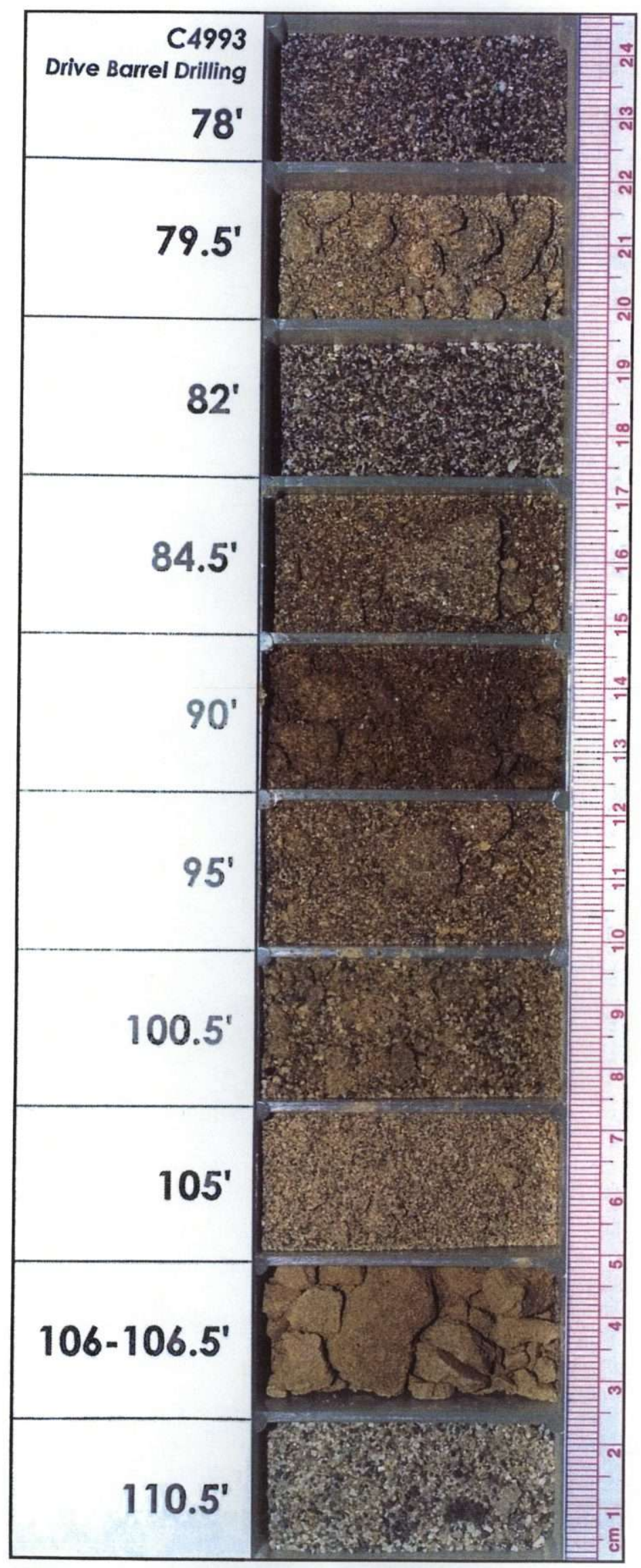

E-3 
WMP-32119, Rev. 0

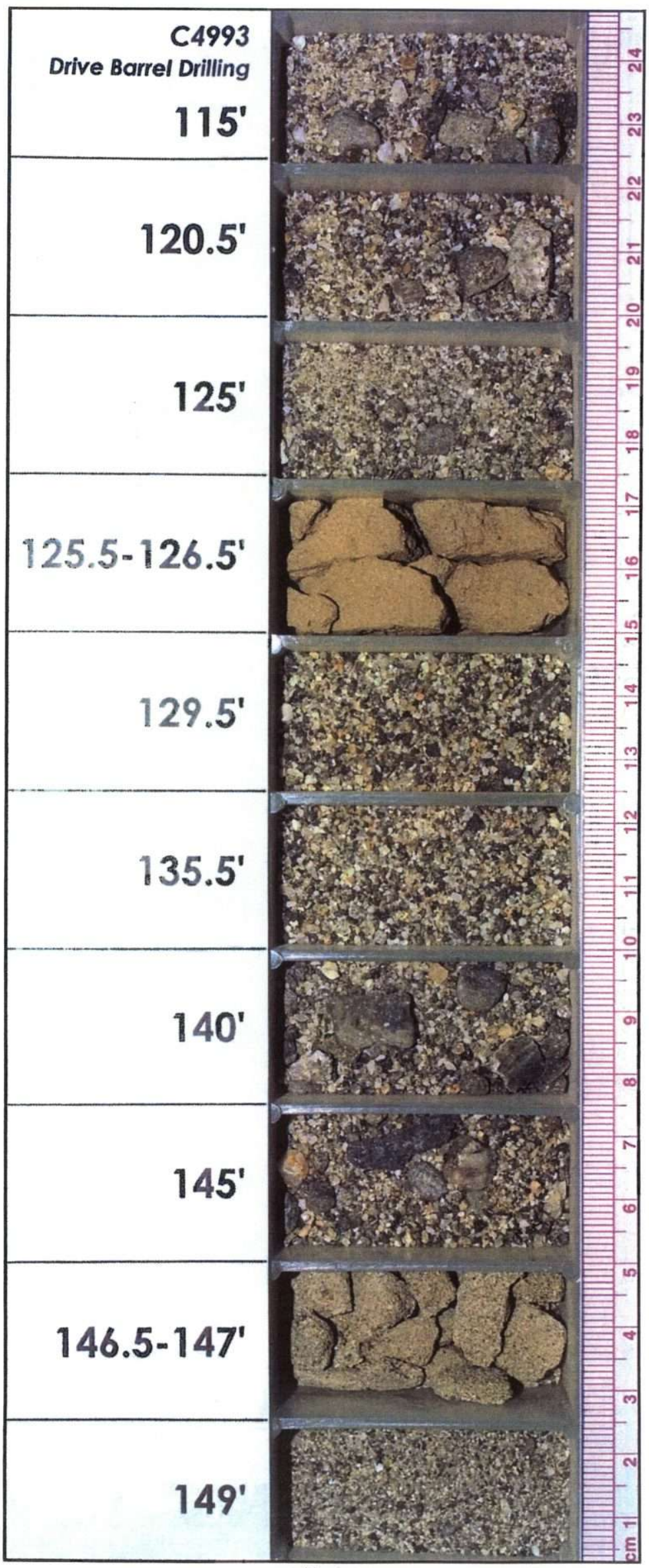

E-4 
WMP-32119, Rev. 0

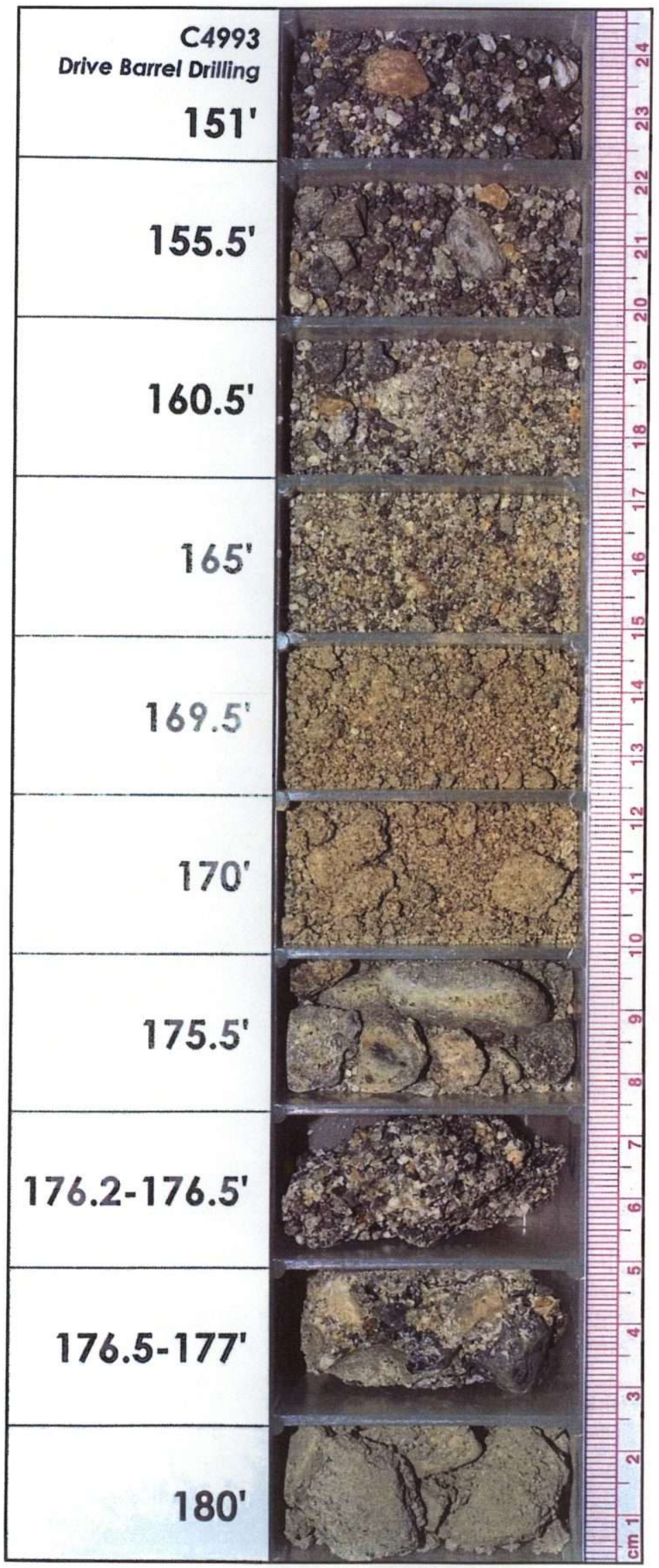

E-5 
WMP-32119, Rev. 0

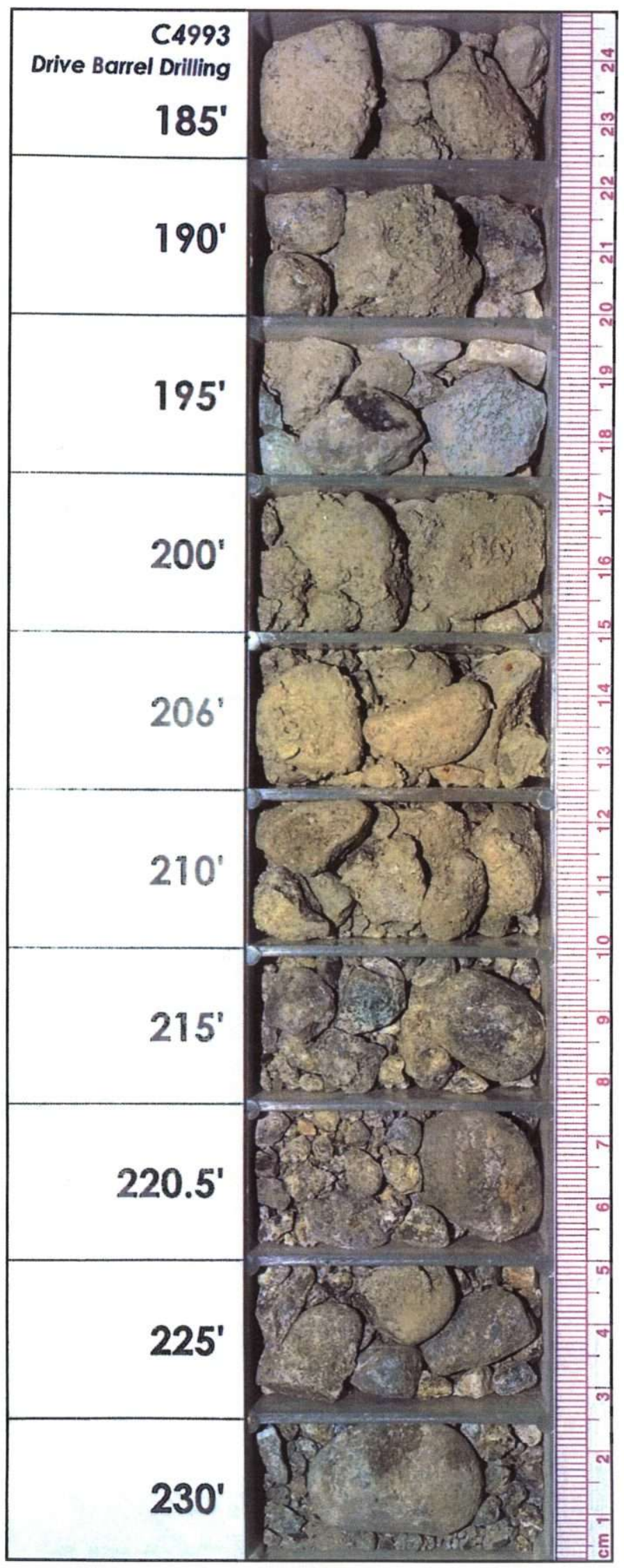


WMP-32119, Rev. 0

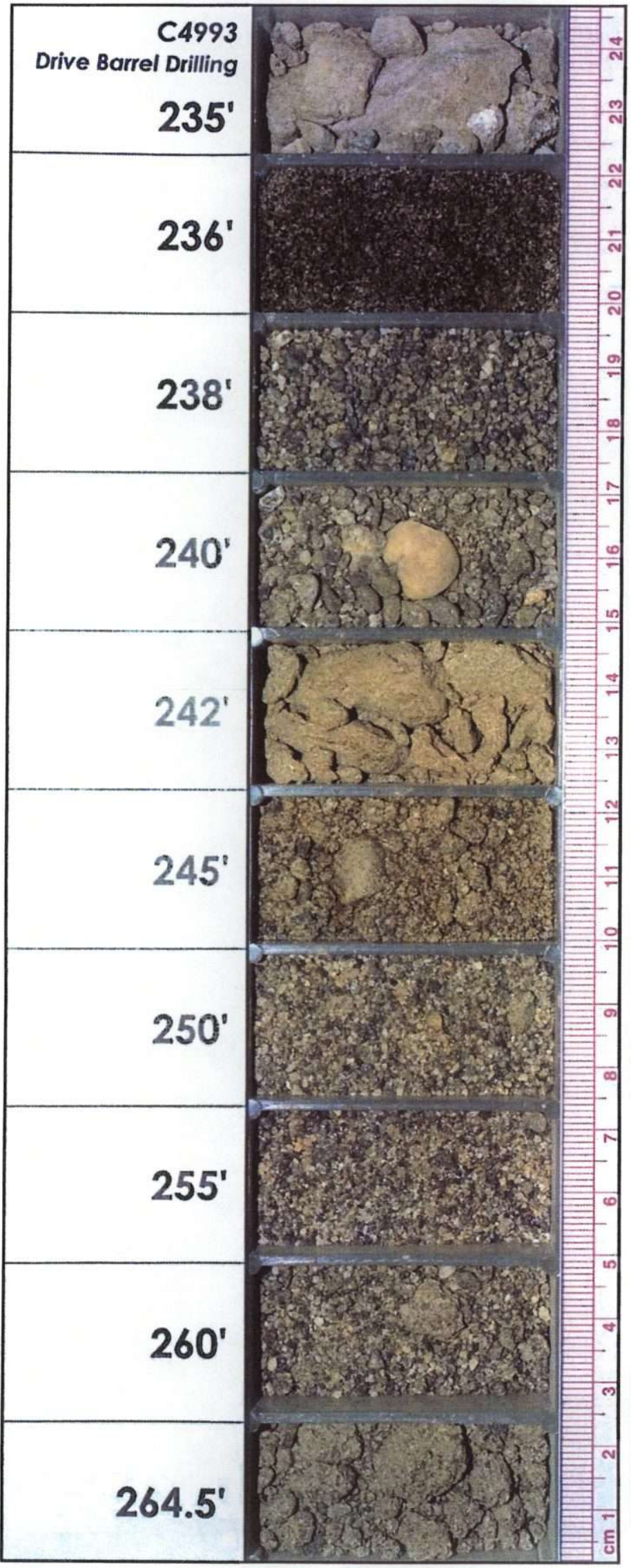

E-7 
WMP-32119, Rev. 0

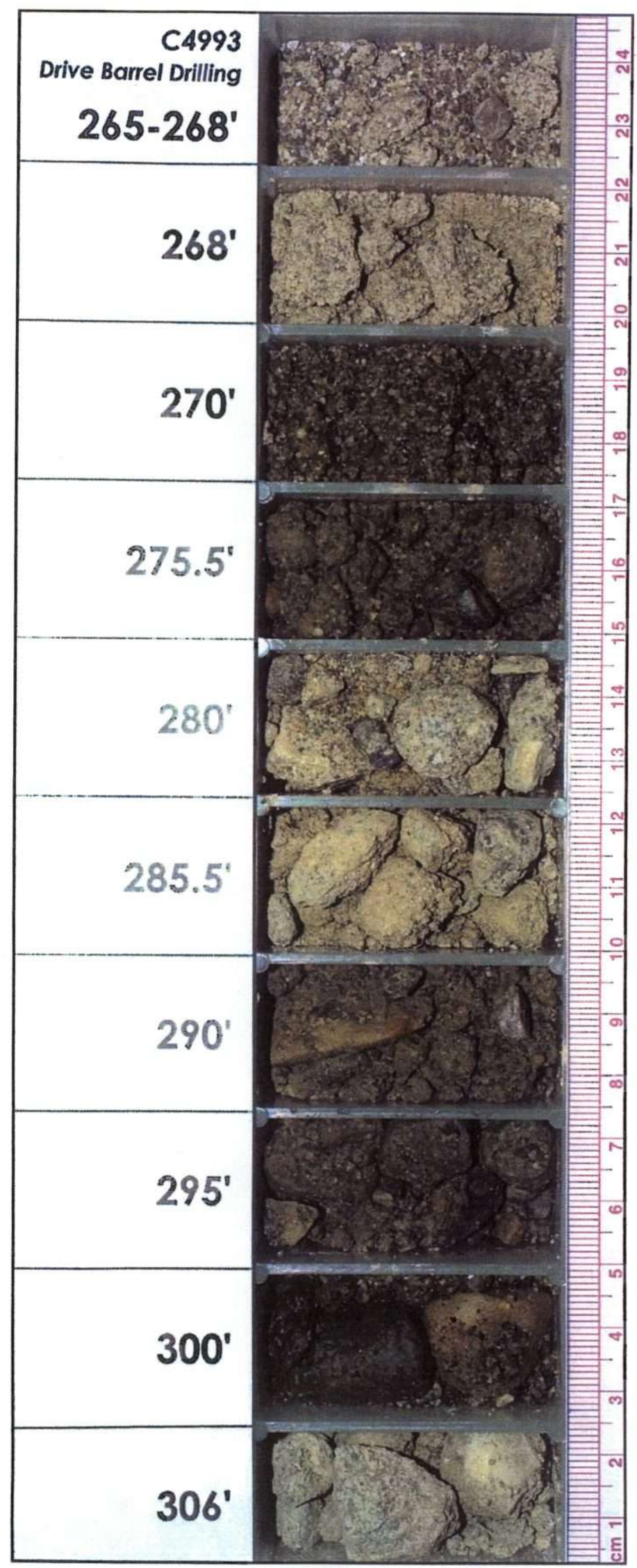

E-8 
WMP-32119, Rev. 0

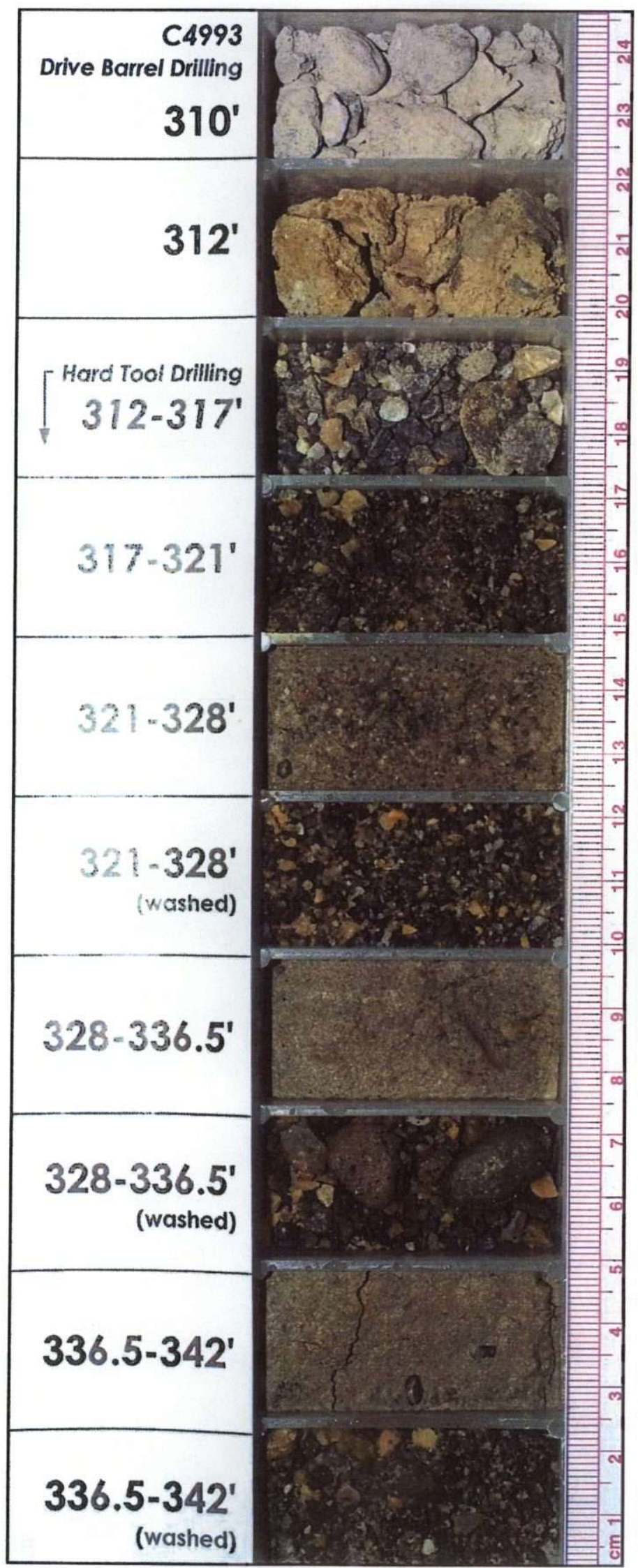


WMP-32119, Rev. 0

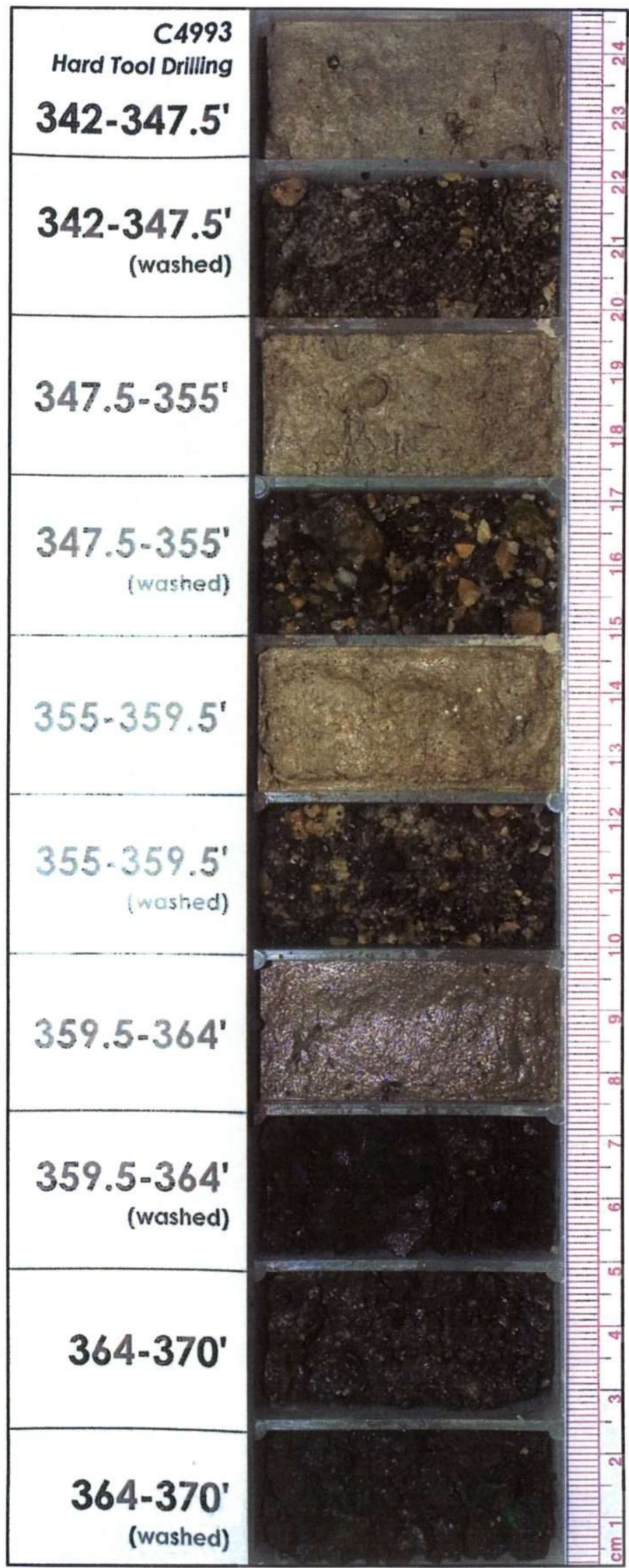

E-10 
WMP-32119, Rev. 0

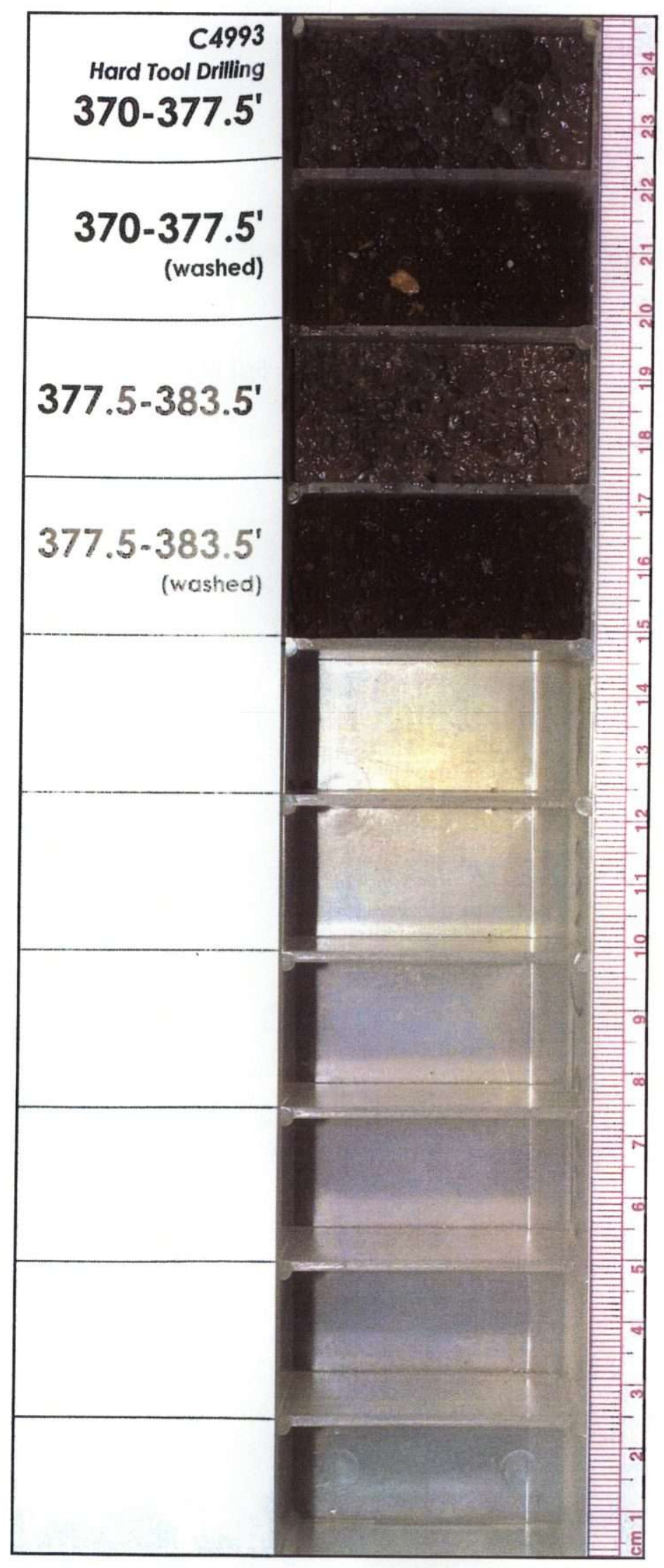


WMP-32119, Rev. 0

This page intentionally left blank. 
WMP-32119, Rev. 0

APPENDIX F

CHIP TRAY PHOTOGRAPHS (C4996) 
WMP-32119, Rev. 0

This page intentionally left blank.

F-ii 
WMP-32119, Rev. 0

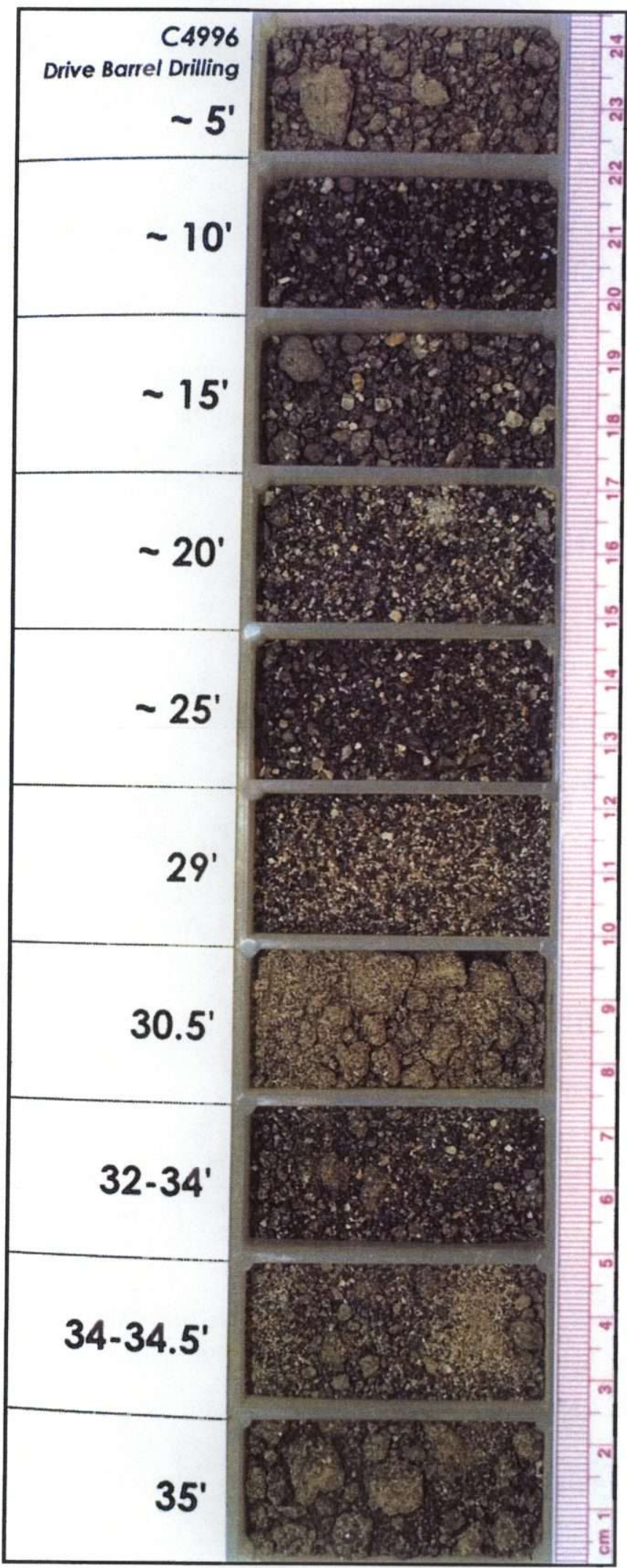

F-1 
WMP-32119, Rev. 0

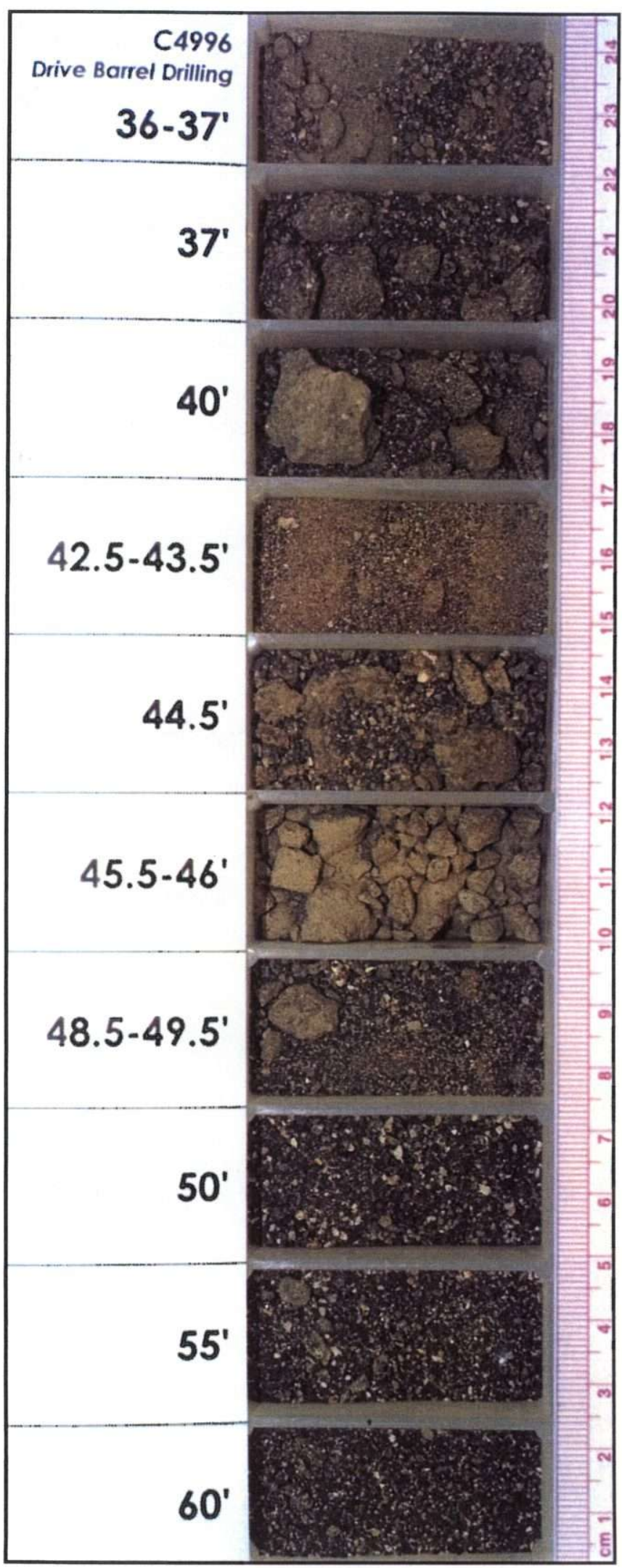


WMP-32119, Rev. 0

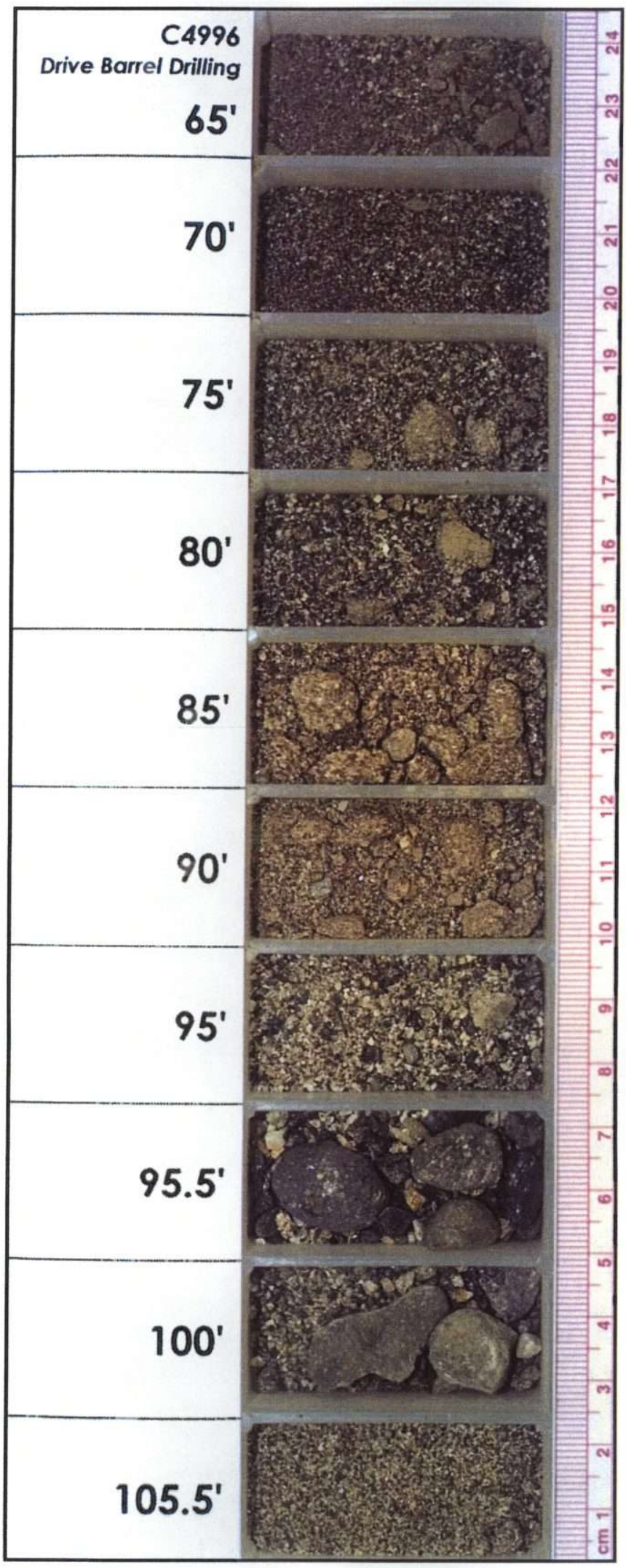

F-3 
WMP-32119, Rev. 0

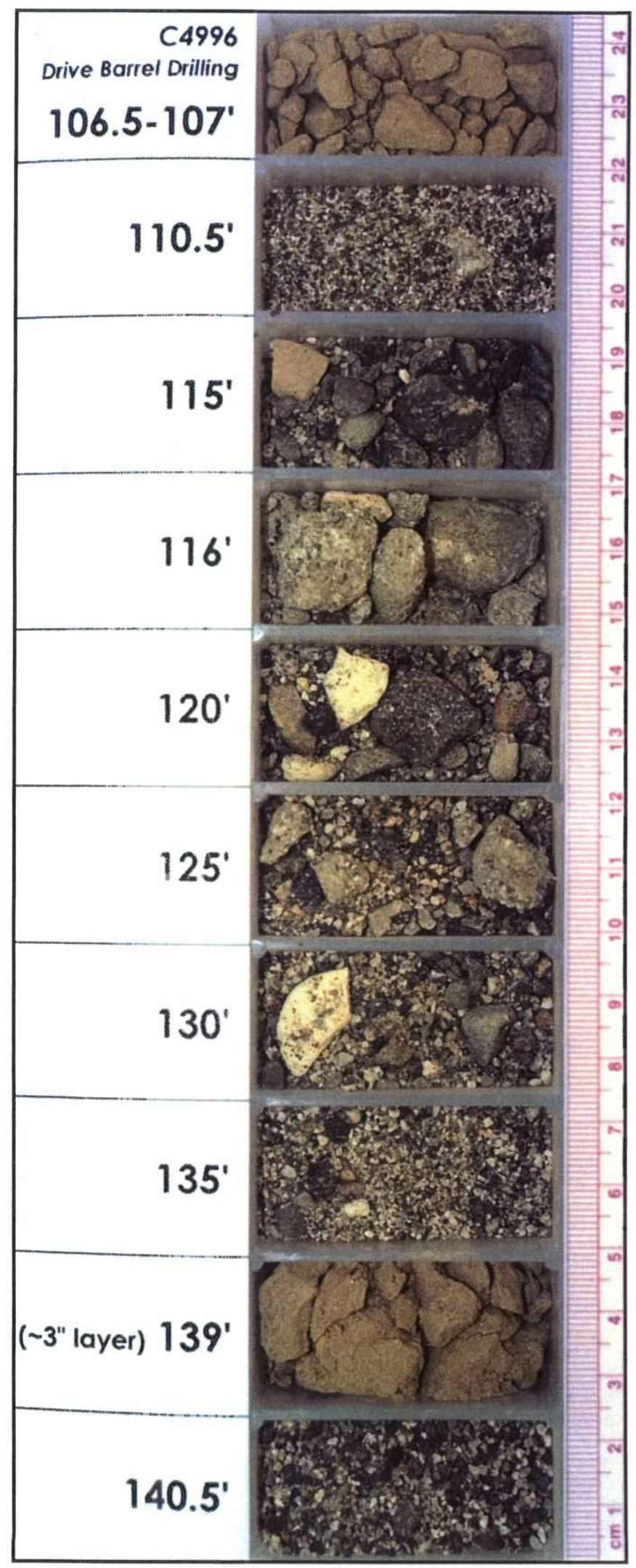


WMP-32119, Rev. 0

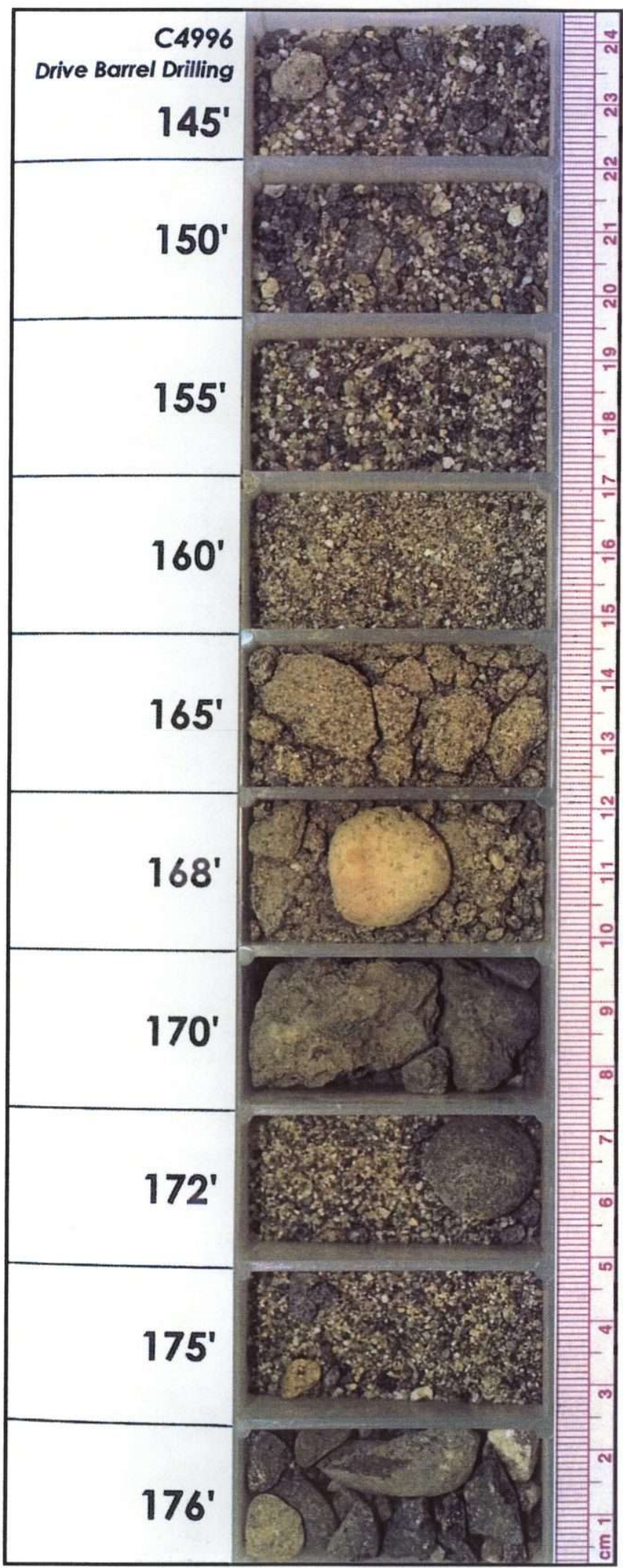


WMP-32119, Rev. 0

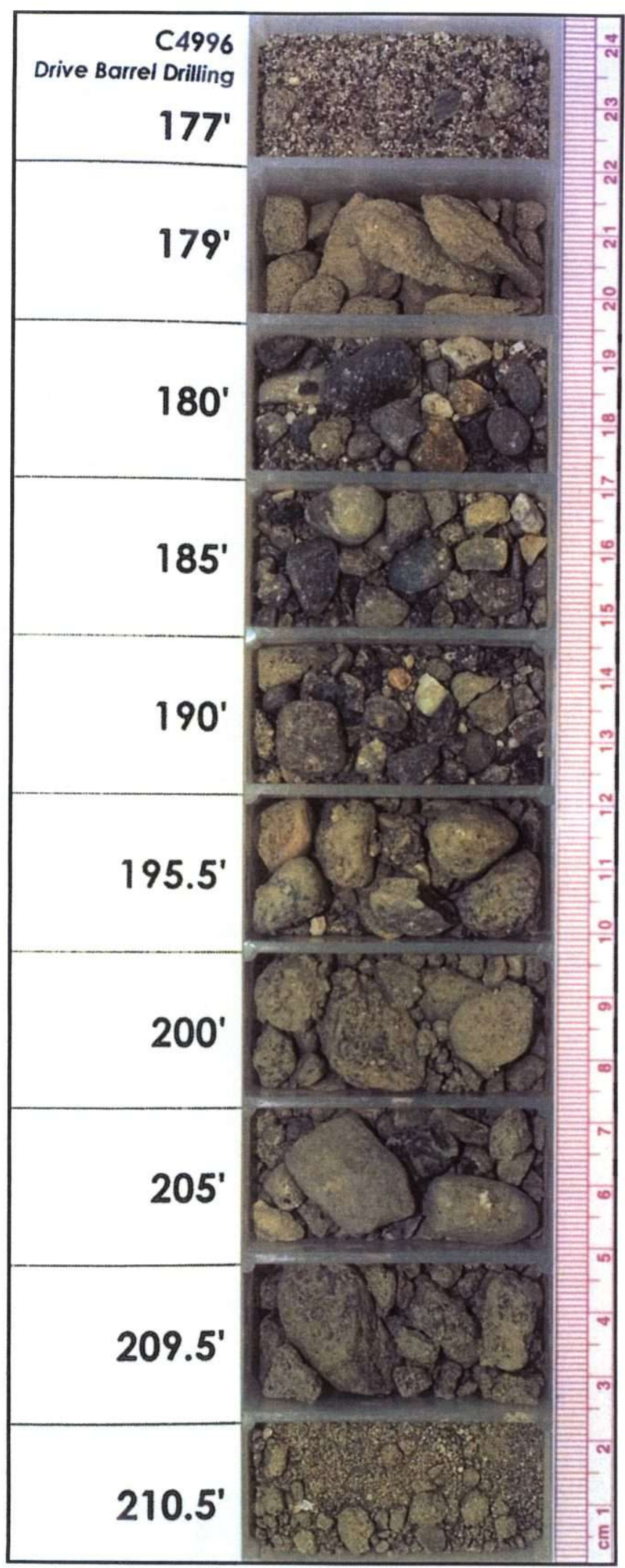


WMP-32119, Rev. 0

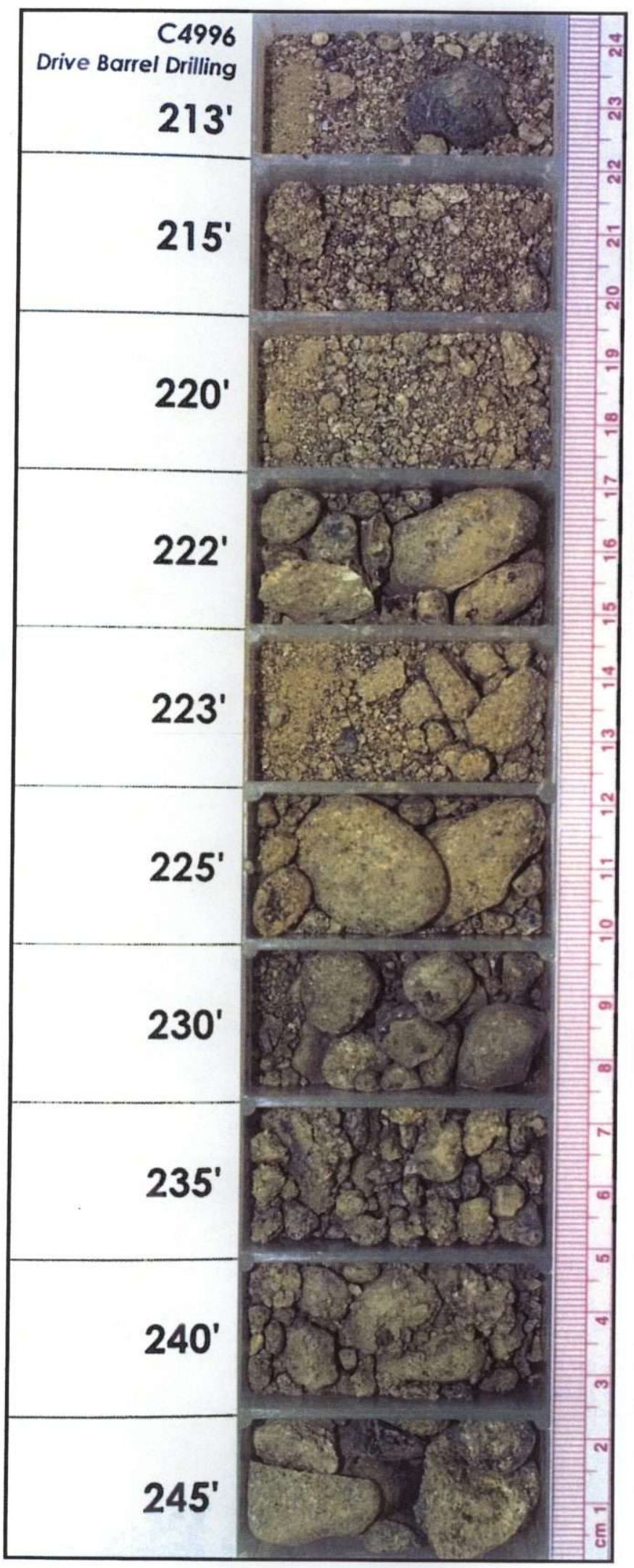

F-7 
WMP-32119, Rev. 0

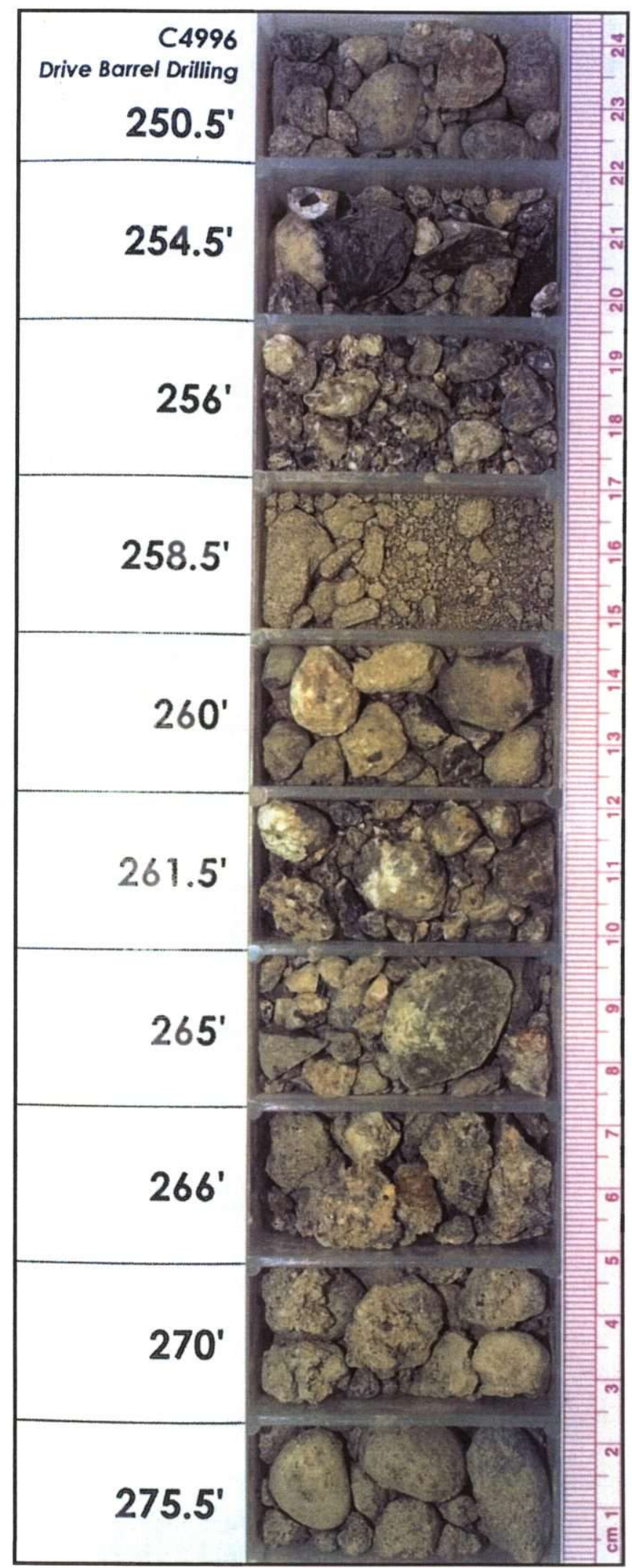


WMP-32119, Rev. 0

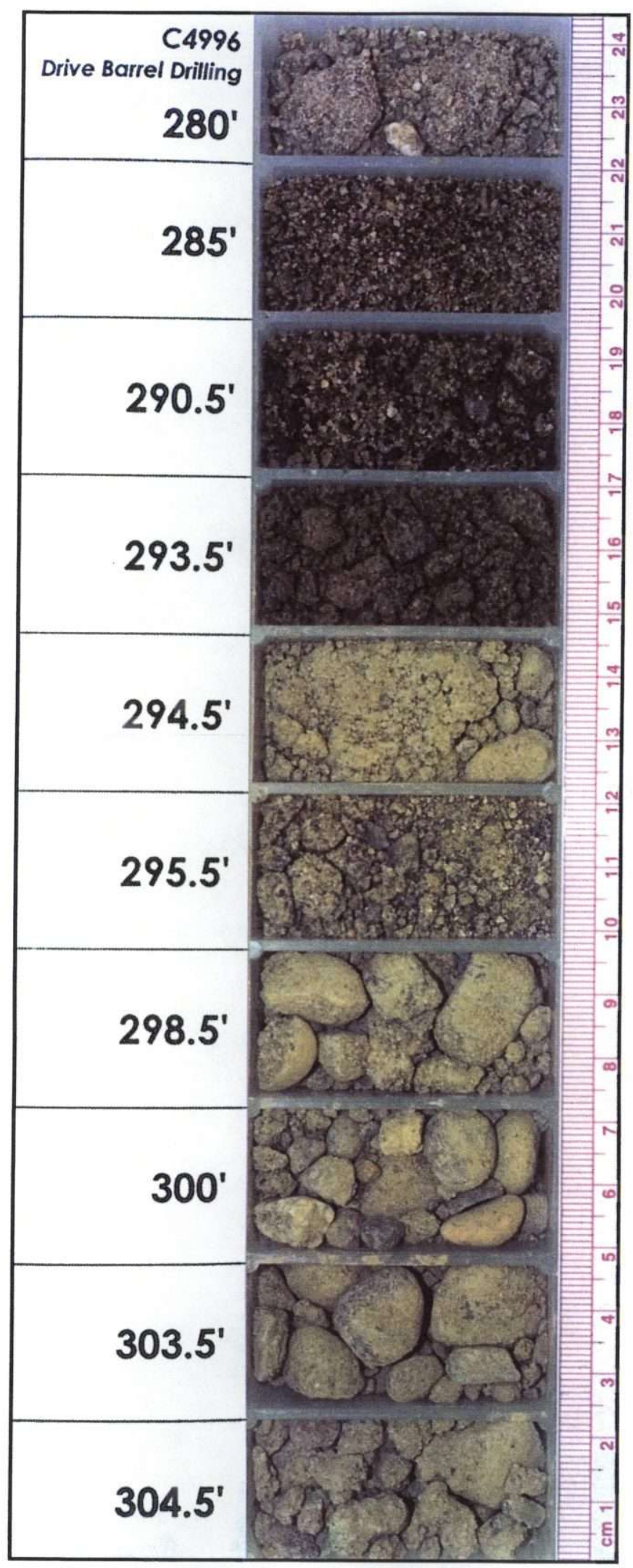


WMP-32119, Rev. 0

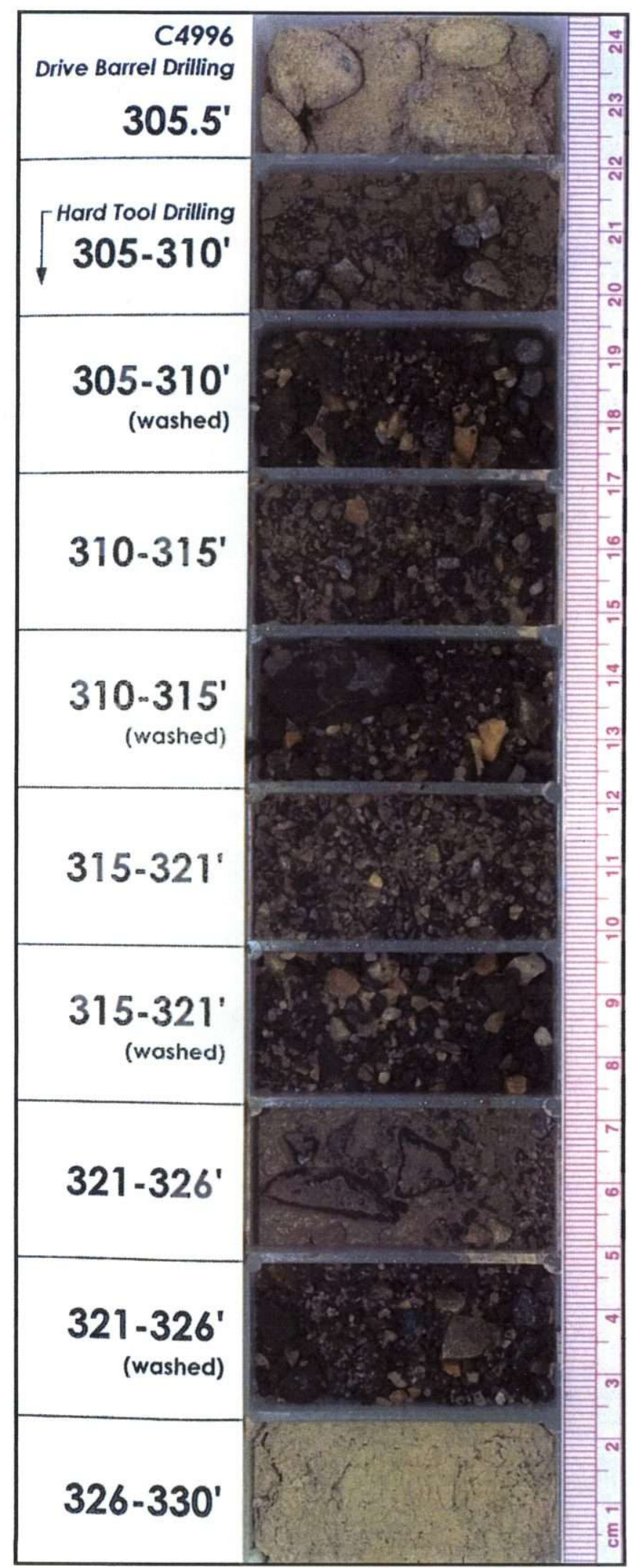


WMP-32119, Rev. 0

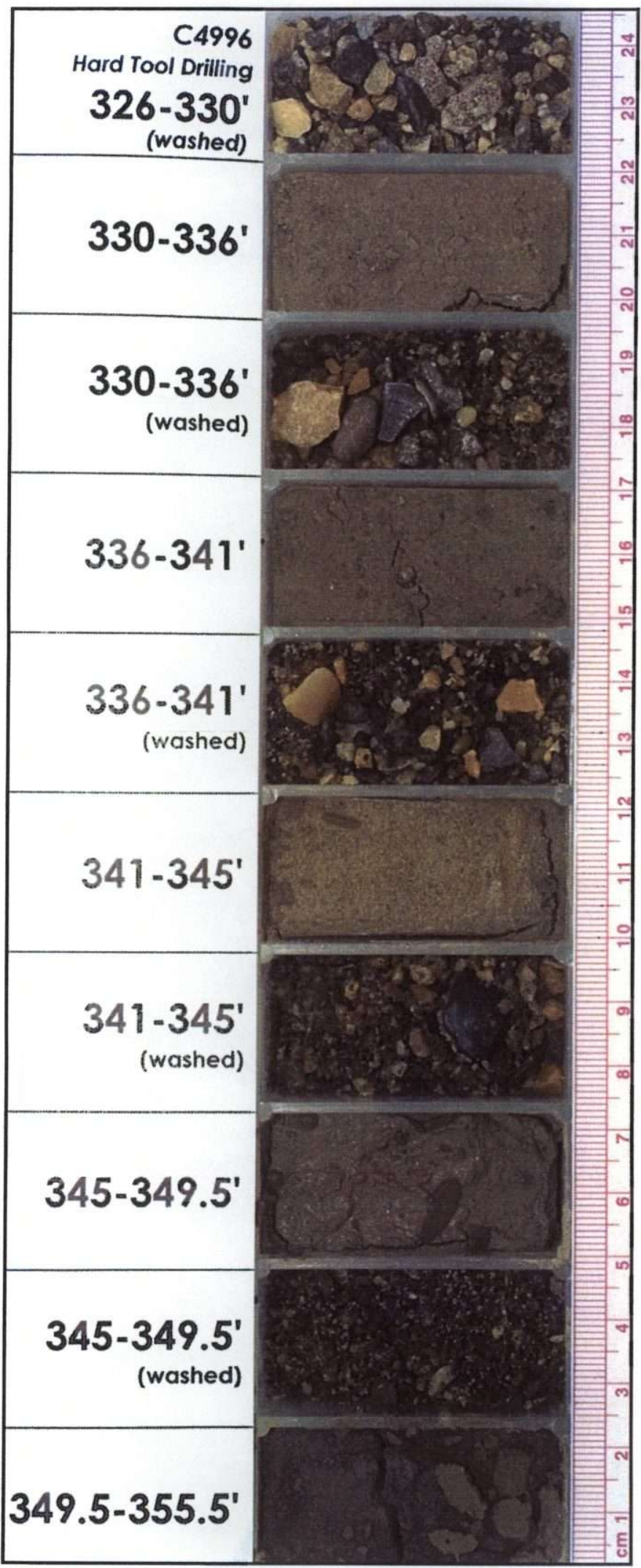


WMP-32119, Rev. 0

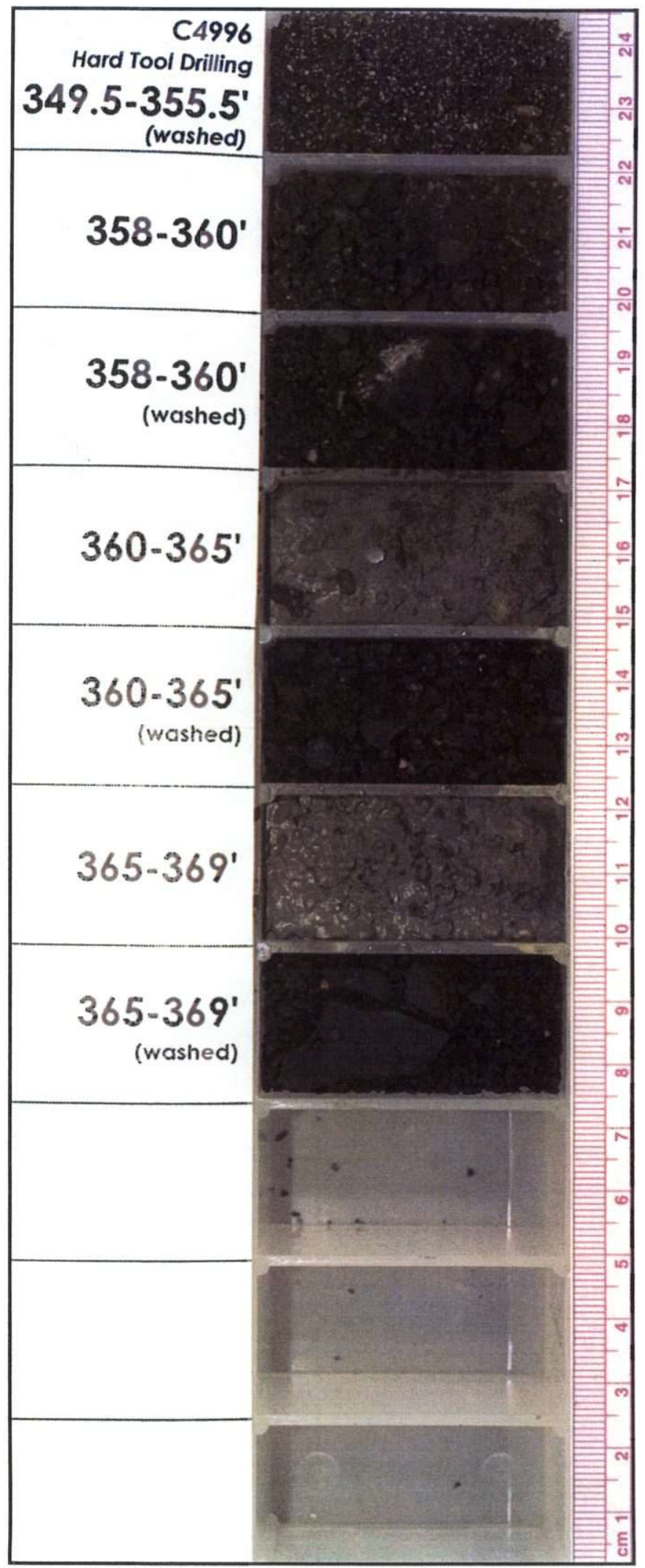


WMP-32119, Rev. 0

APPENDIX G

\section{CHIP TRAY PHOTOGRAPHS (C4997)}

G-i 
WMP-32119, Rev. 0

3

G-ii 
WMP-32119, Rev. 0

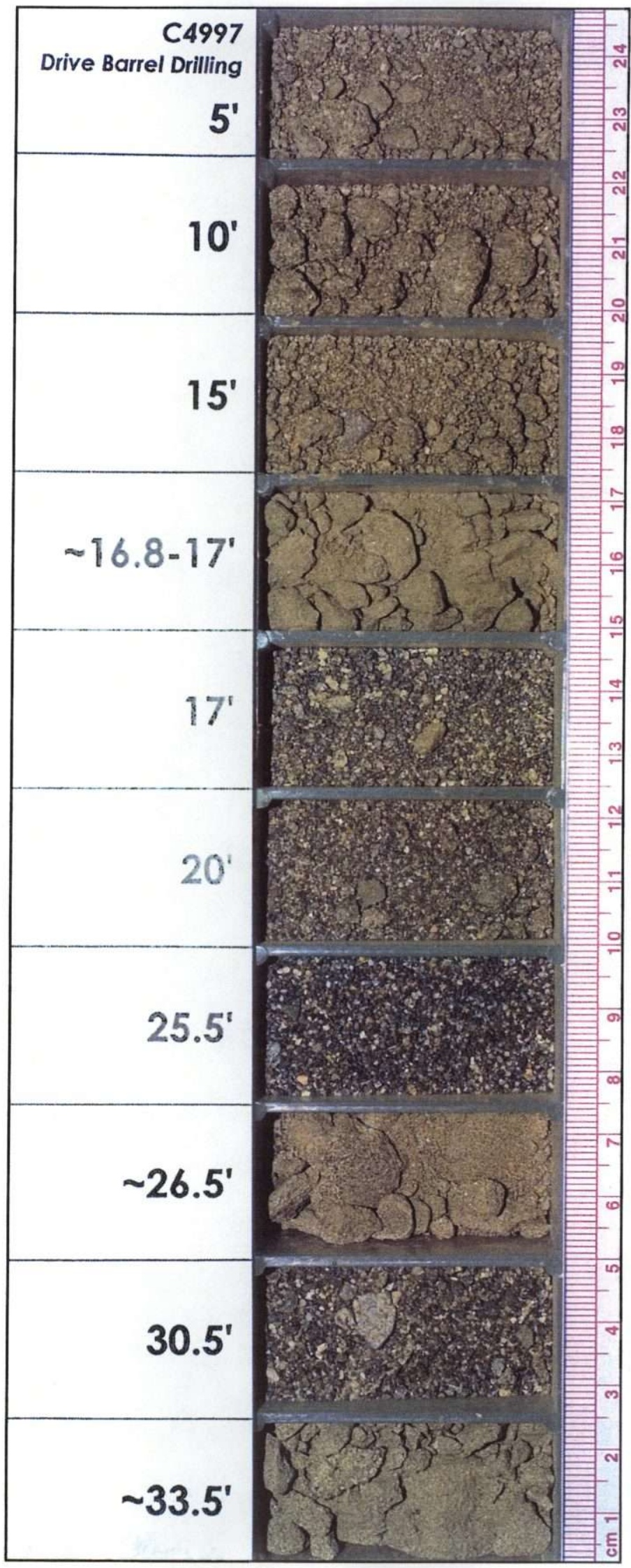

G-1 
WMP-32119, Rev. 0

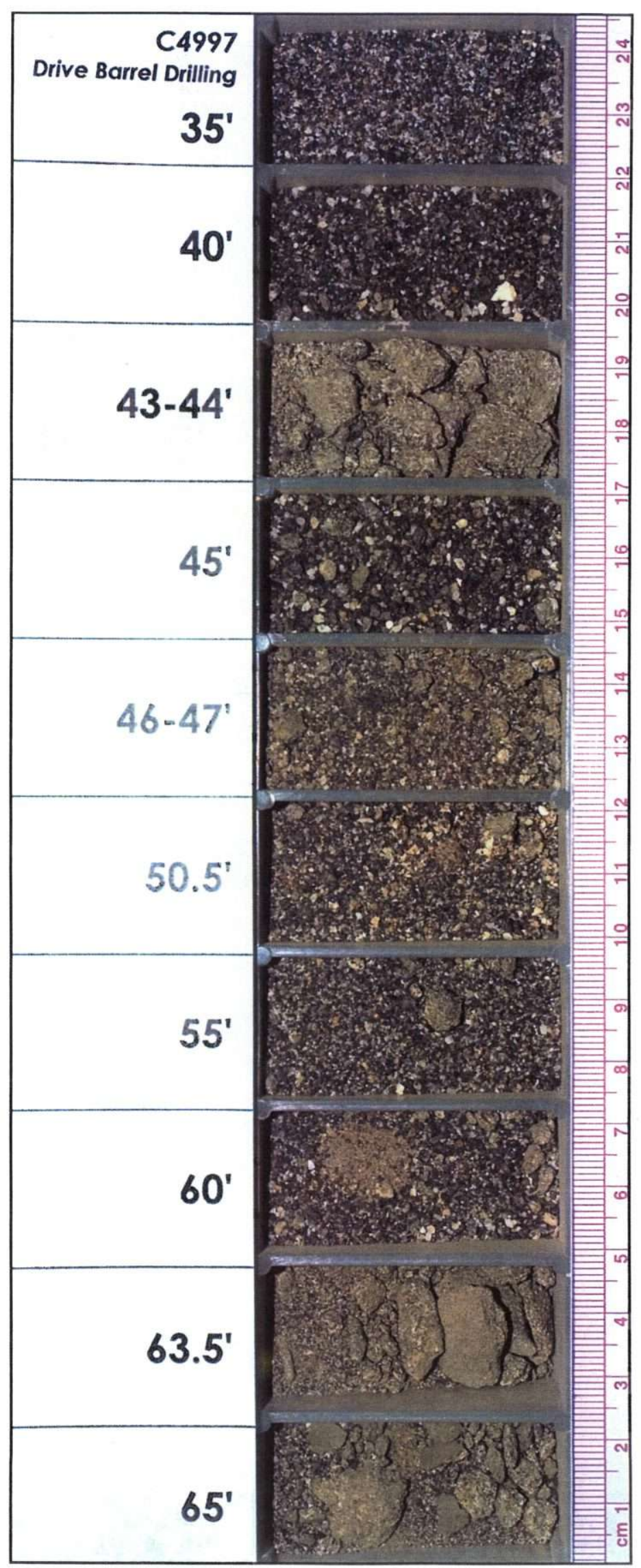


WMP-32119, Rev. 0

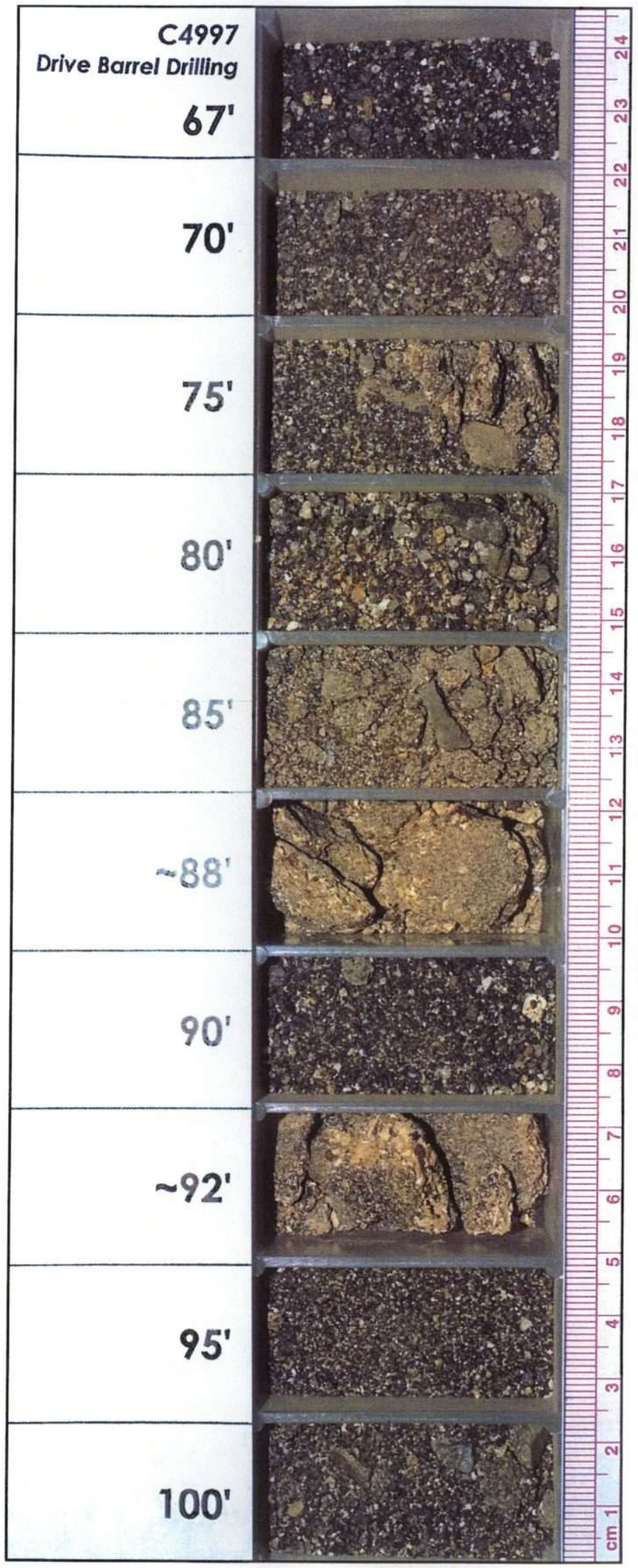

G-3 
WMP-32119, Rev. 0

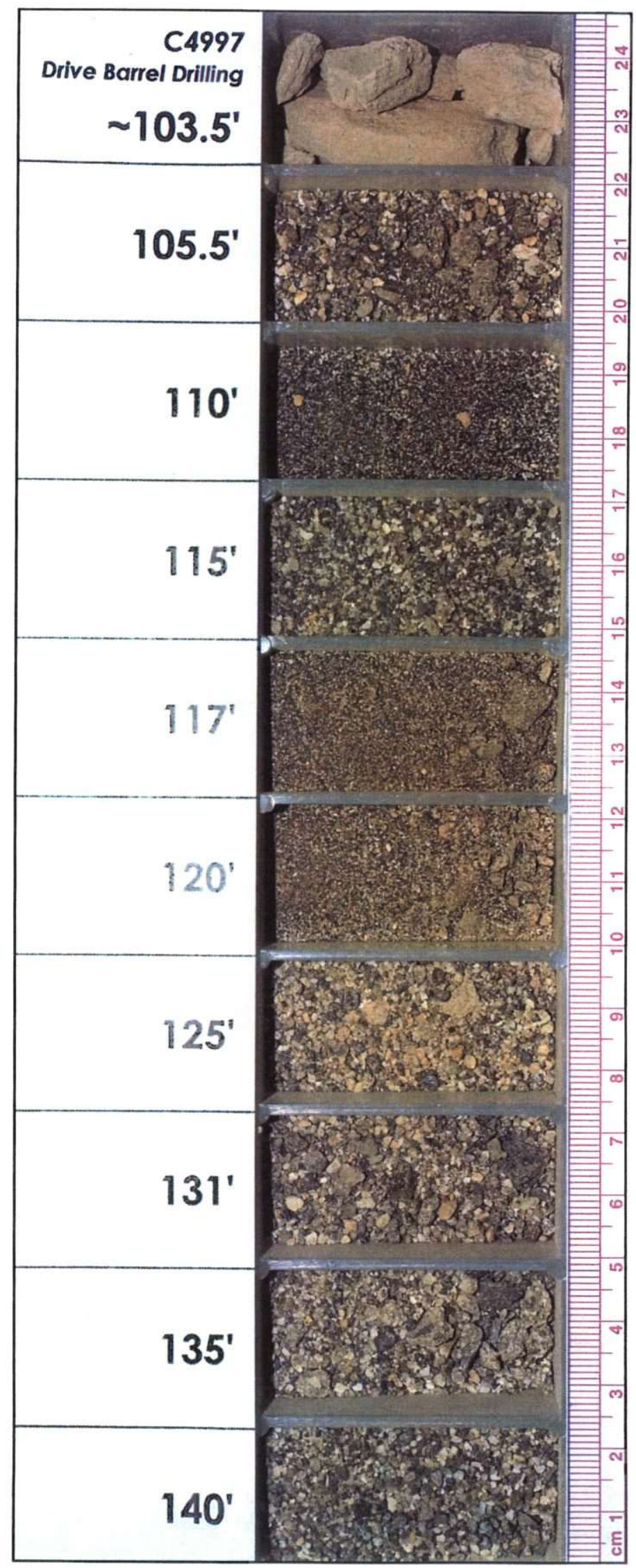

G-4 
WMP-32119, Rev. 0

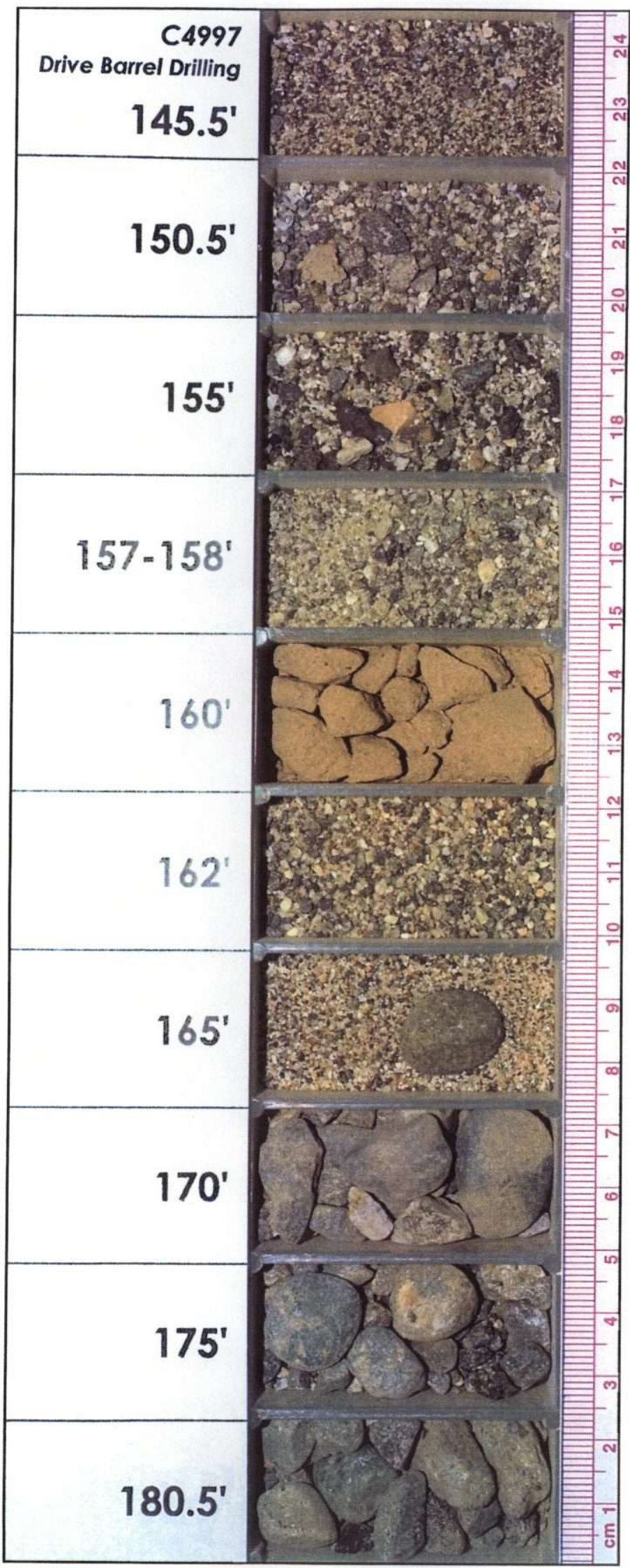

G-5 
WMP-32119, Rev. 0

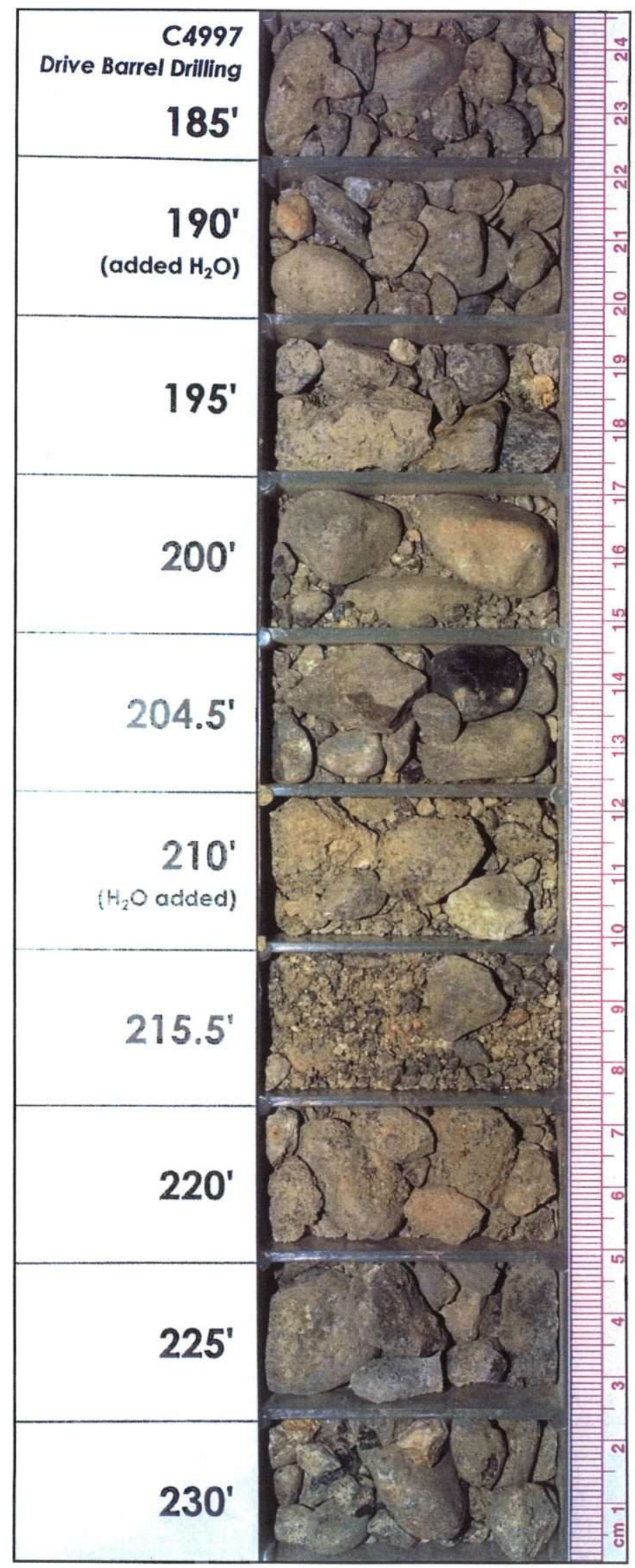

G-6 
WMP-32119, Rev. 0

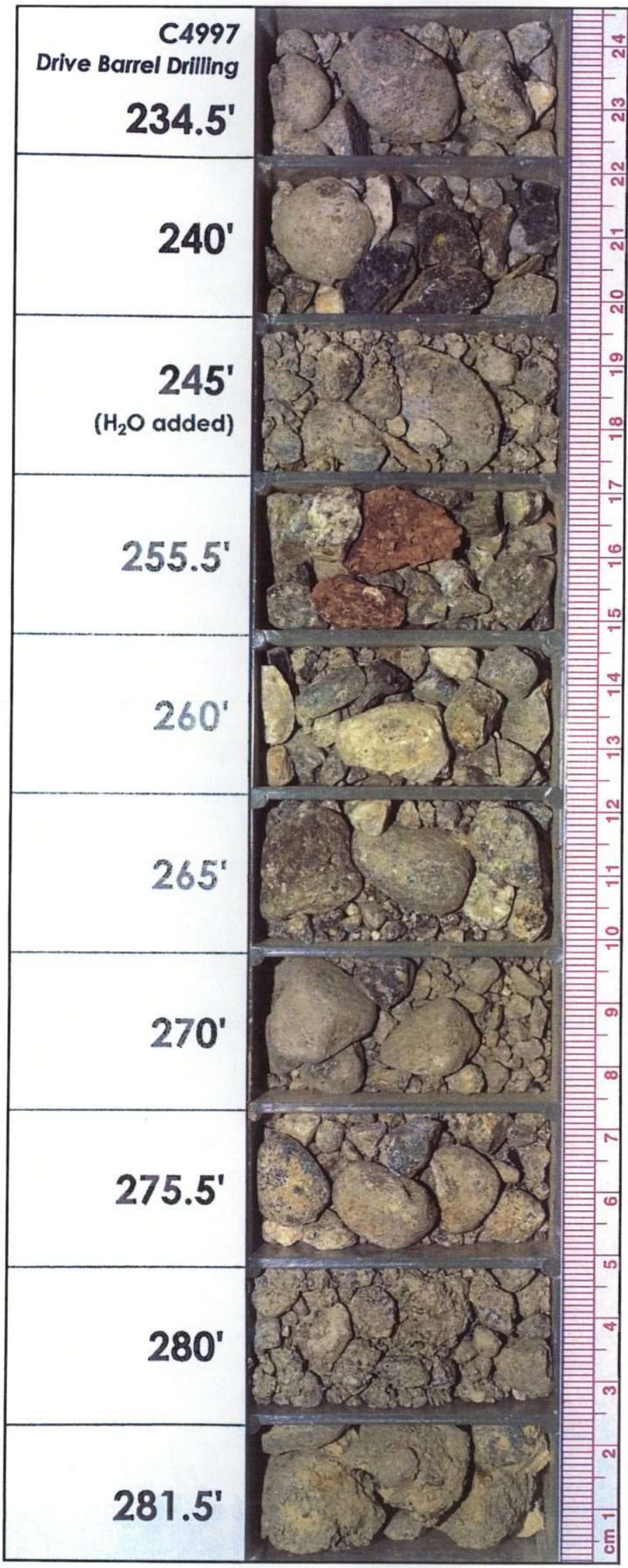

G-7 
WMP-32119, Rev. 0

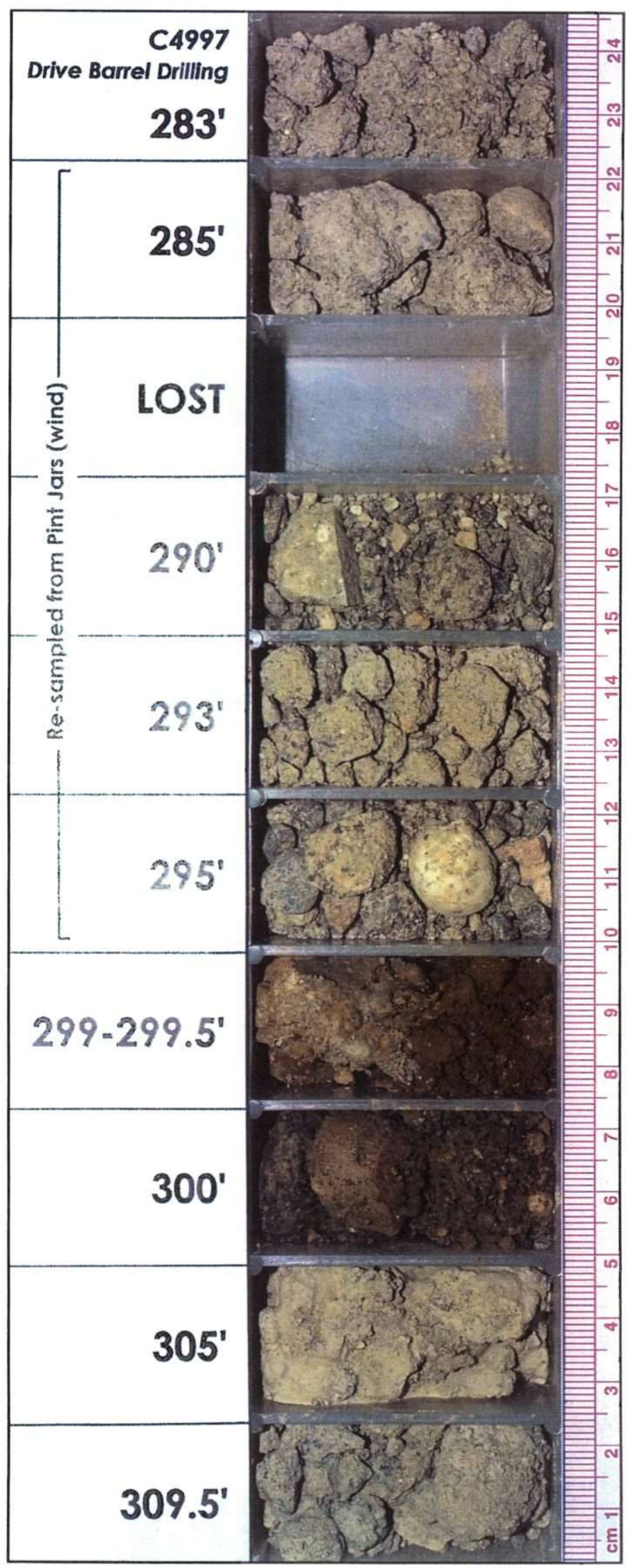


WMP-32119, Rev. 0

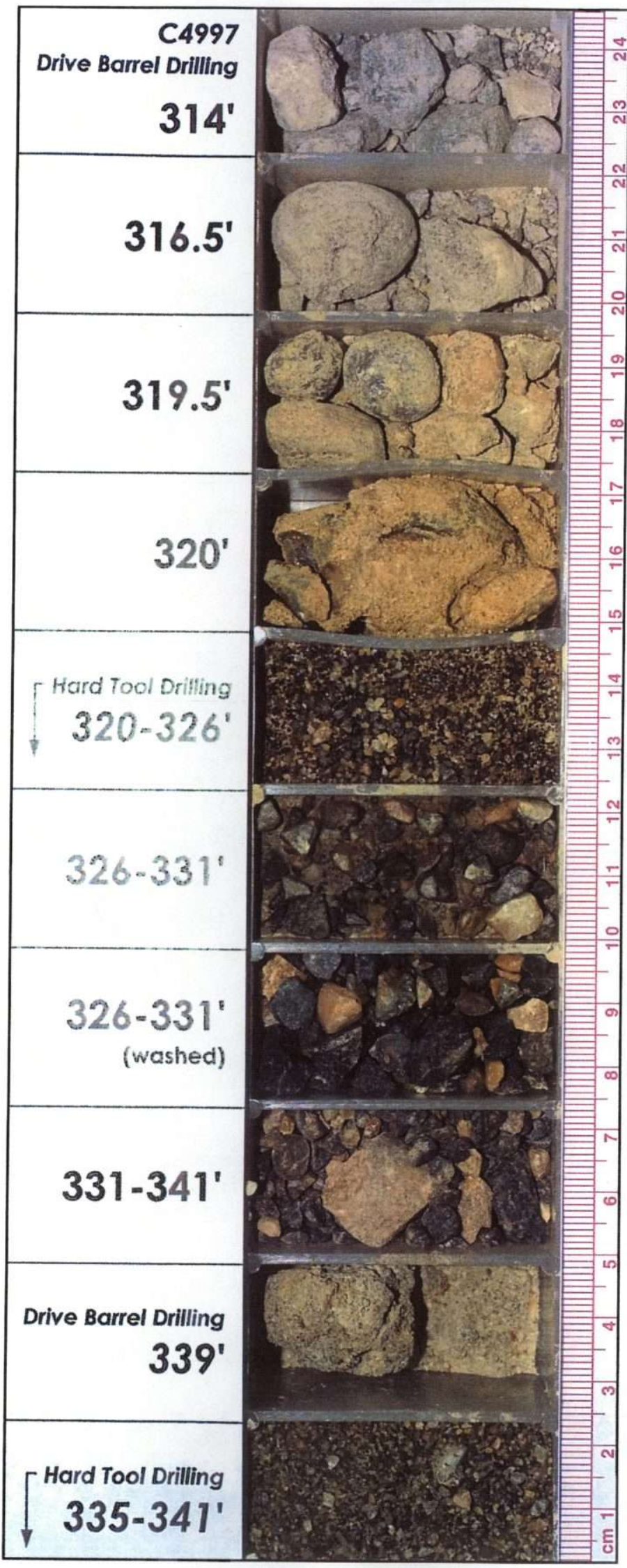

G-9 
WMP-32119, Rev. 0

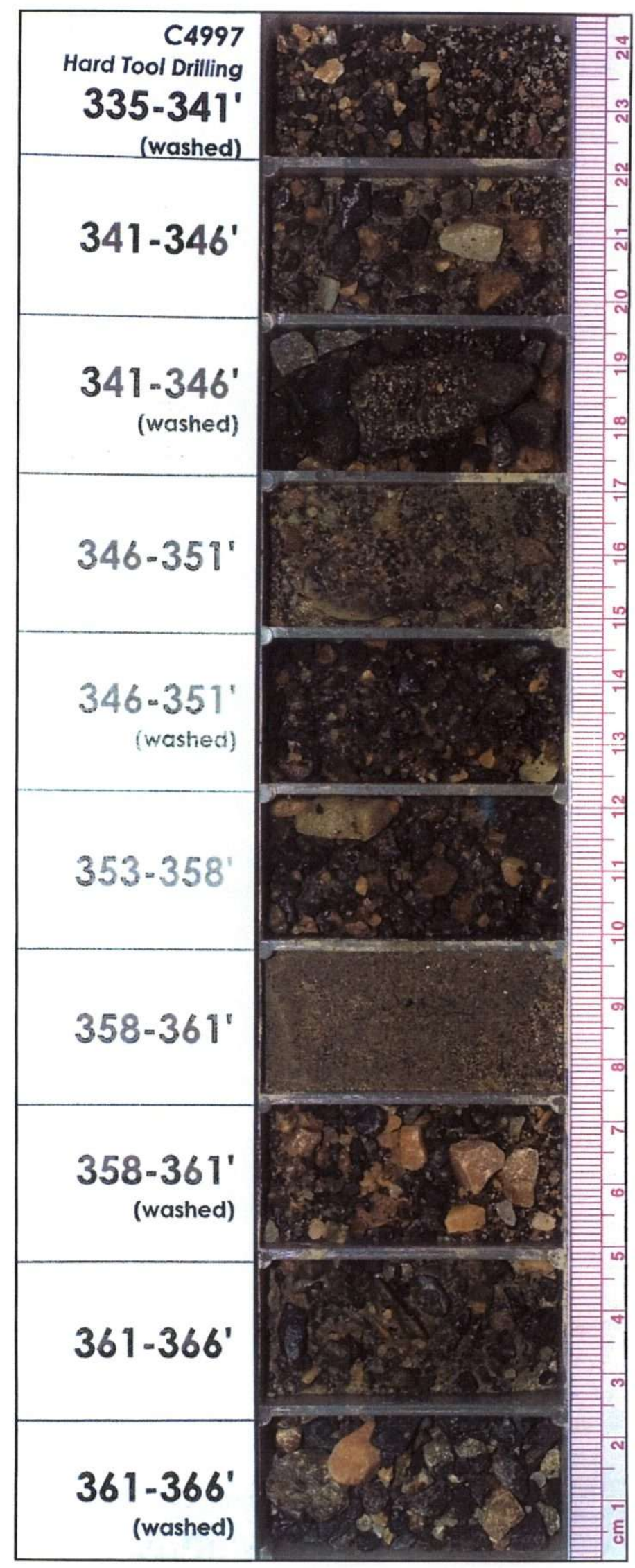


WMP-32119, Rev. 0

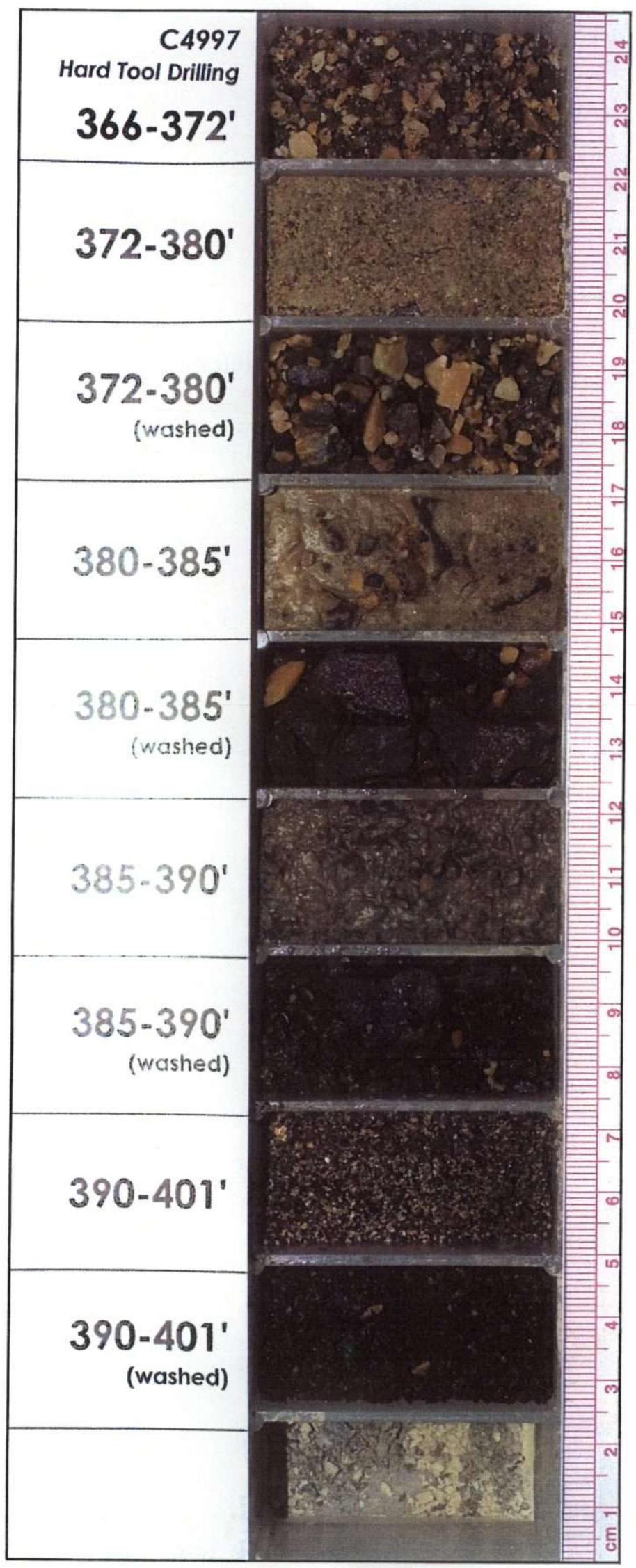

G-11 
WMP-32119, Rev. 0

This page intentionally left blank. 
WMP-32119, Rev. 0

\section{APPENDIX H}

\section{CHIP TRAY PHOTOGRAPHS (C4998)}

H- i 
WMP-32119, Rev. 0

This page intentionally left blank

H- ii 
WMP-32119, Rev. 0

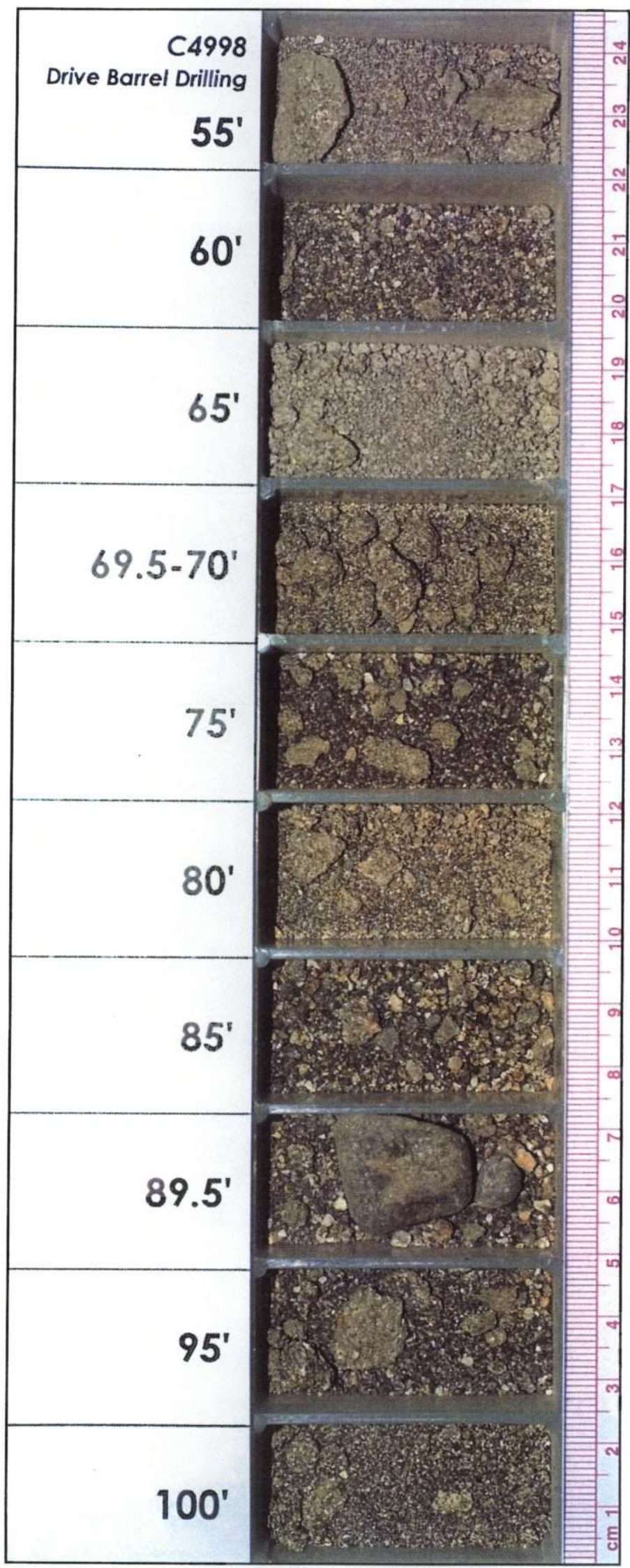

H- 1 
WMP-32119, Rev. 0

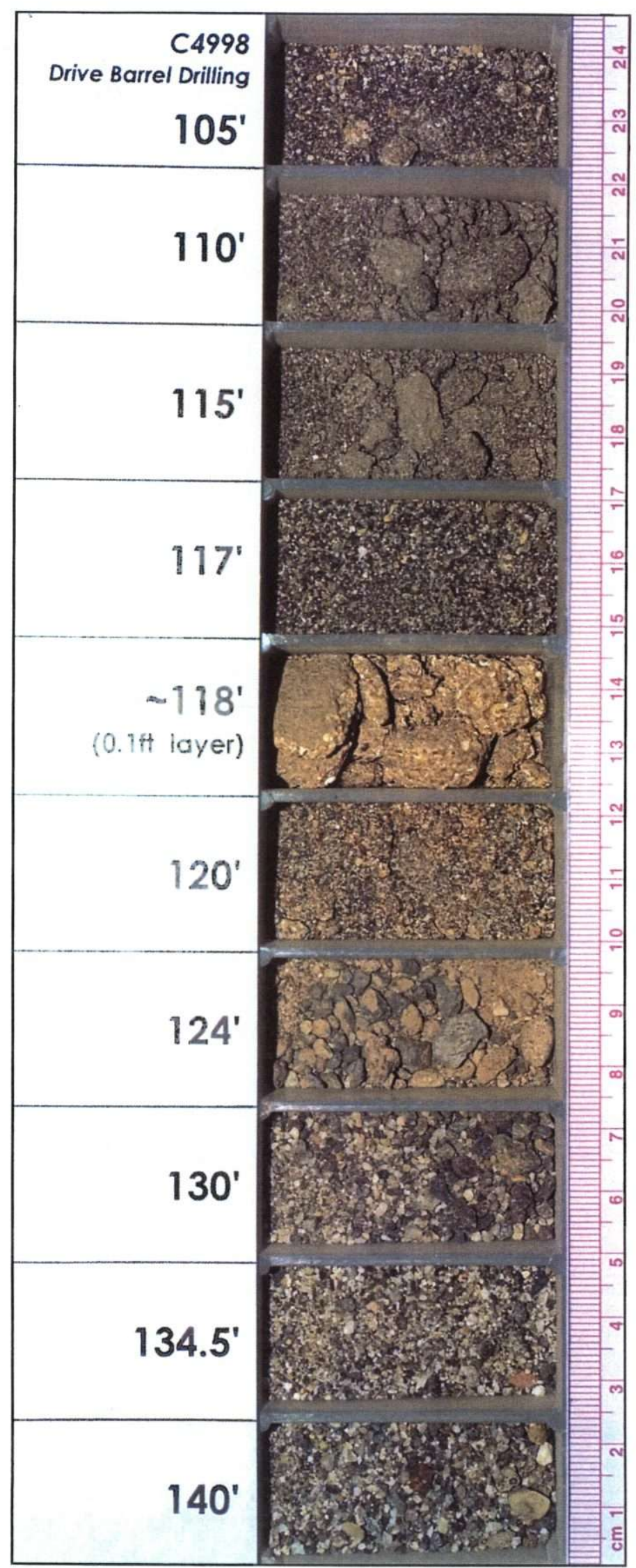

H- 2 
WMP-32119, Rev. 0

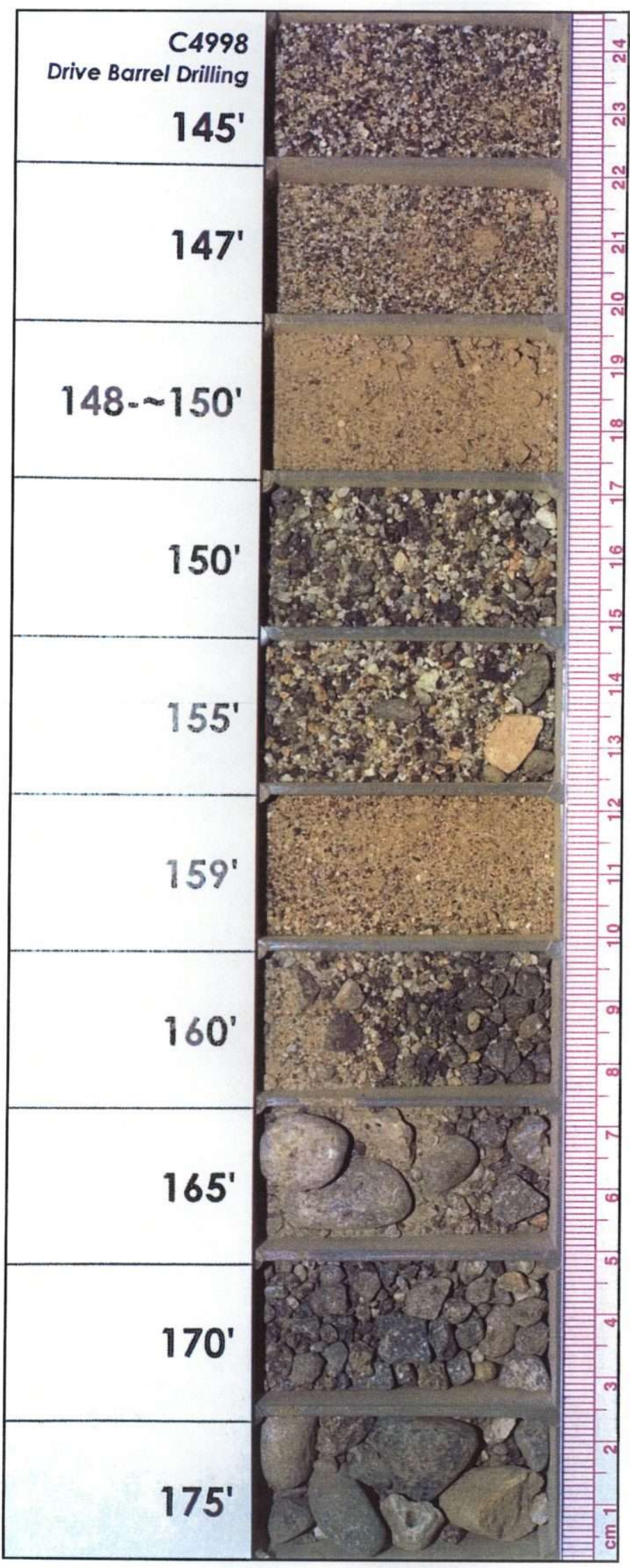

H- 3 
WMP-32119, Rev. 0

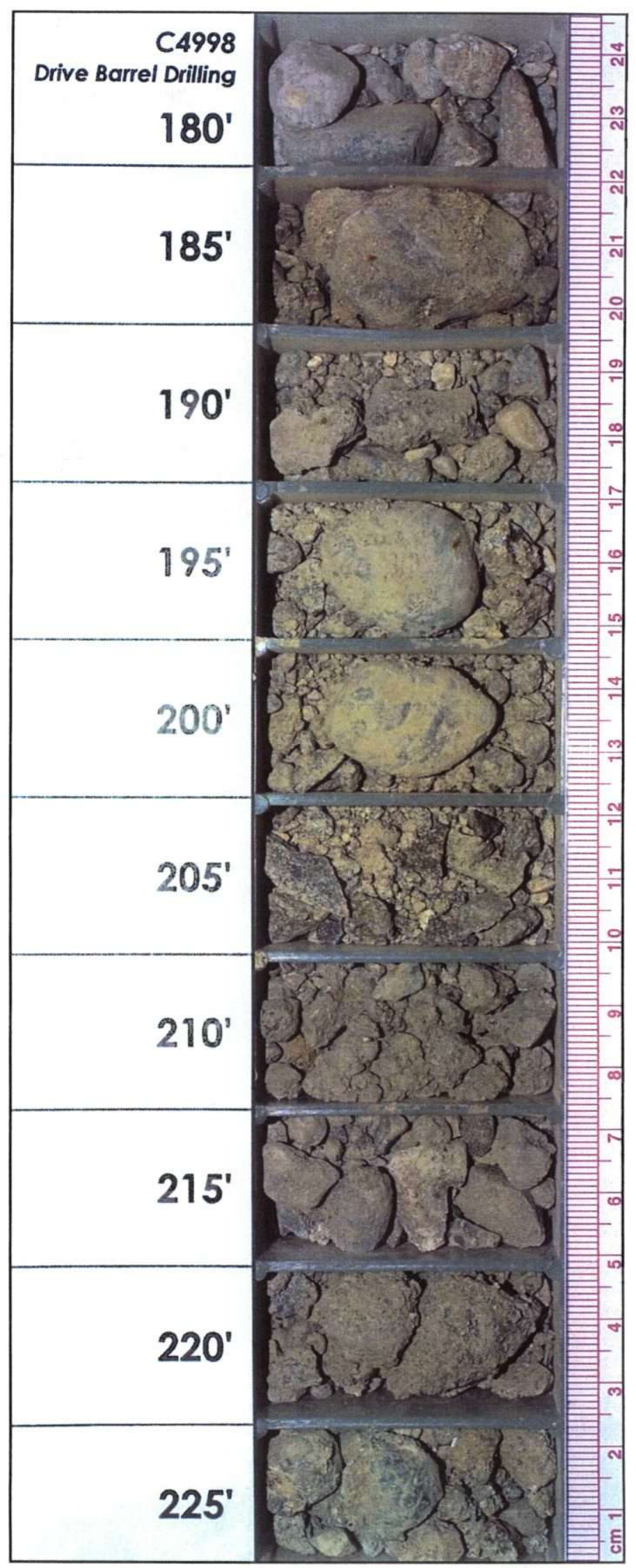

H- 4 
WMP-32119, Rev. 0

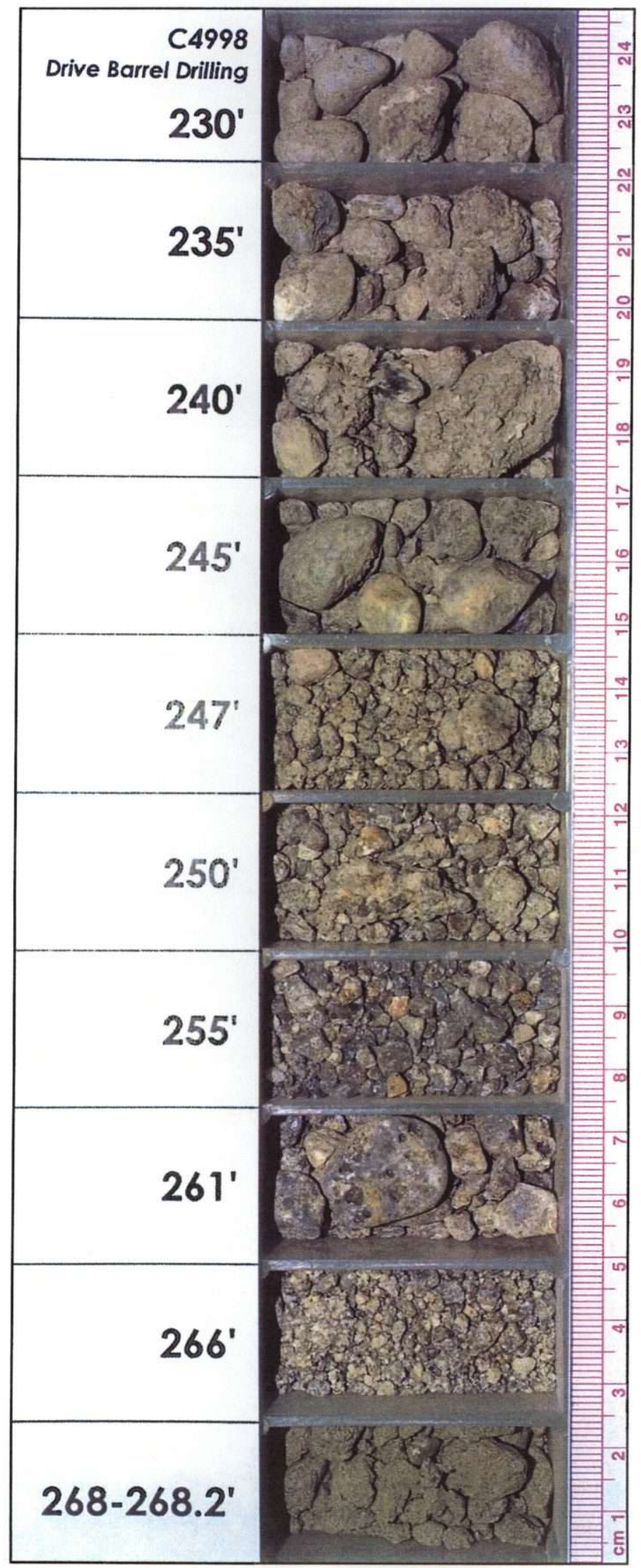

H- 5 
WMP-32119, Rev. 0

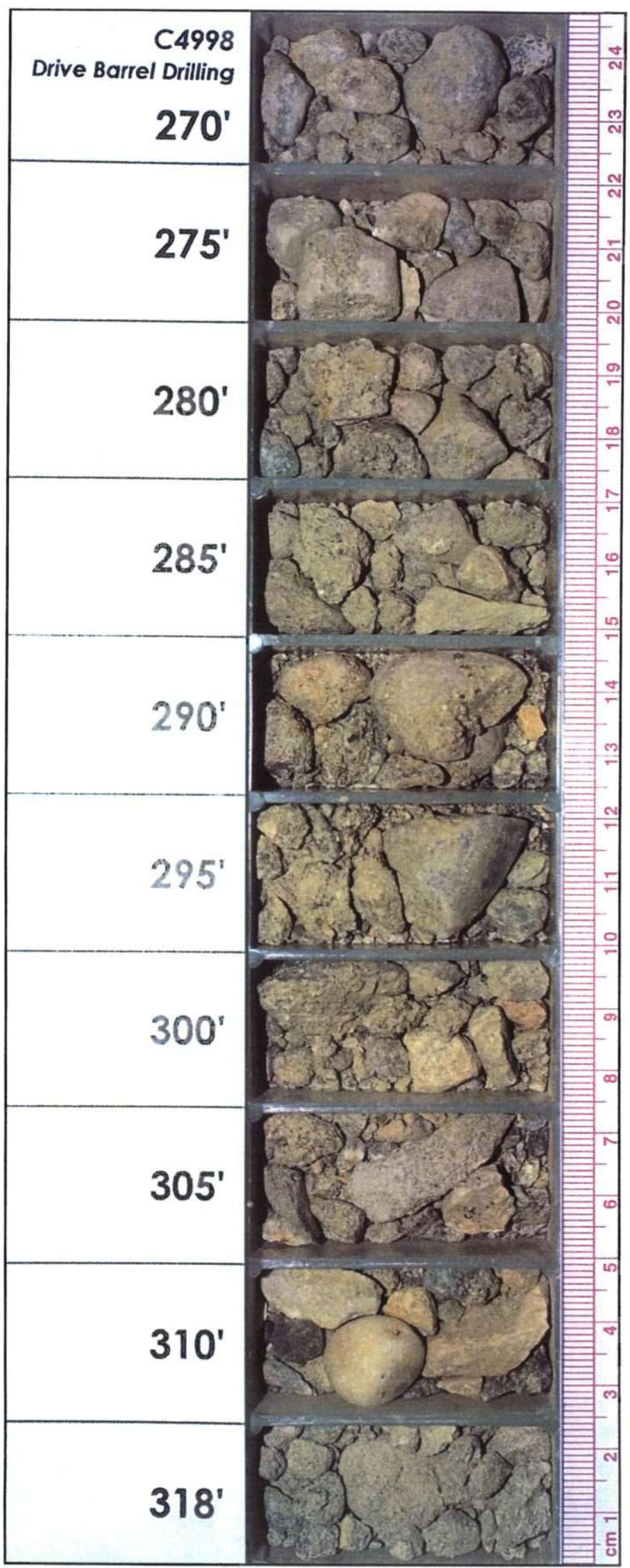

H- 6 
WMP-32119, Rev. 0

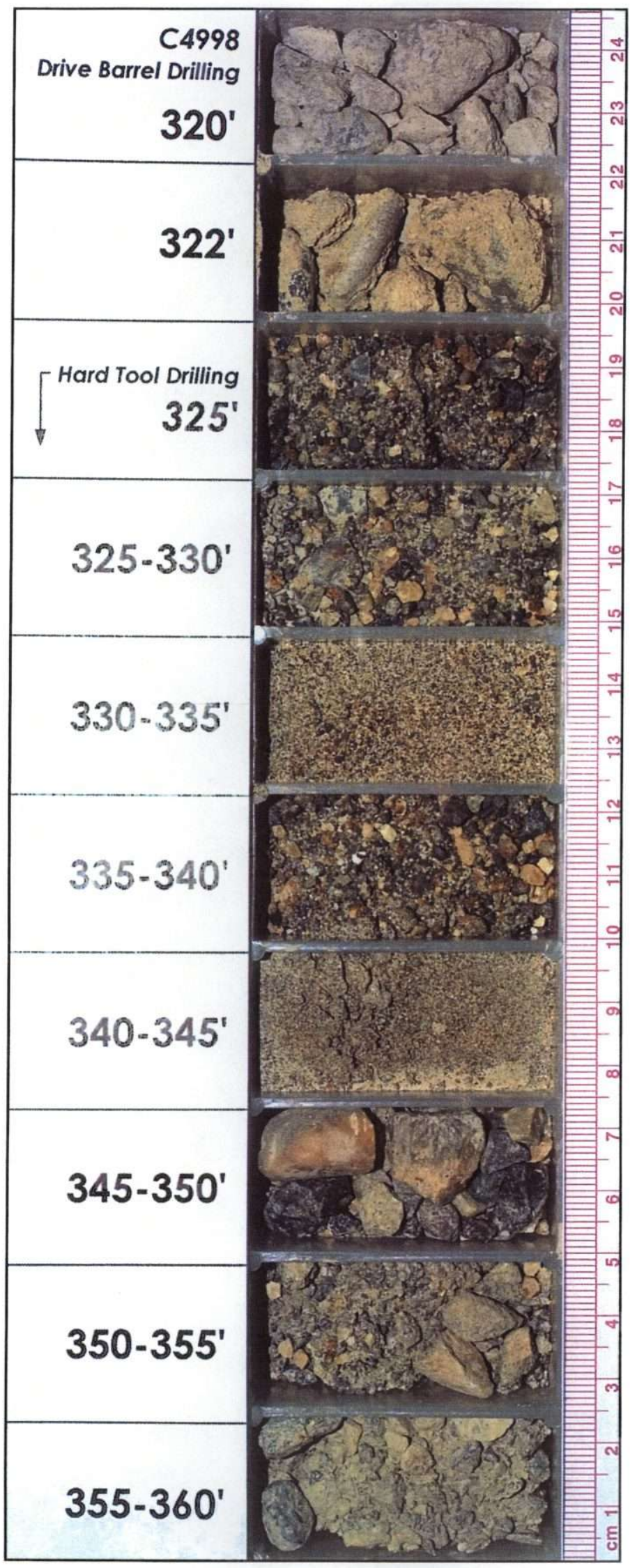


WMP-32119, Rev. 0

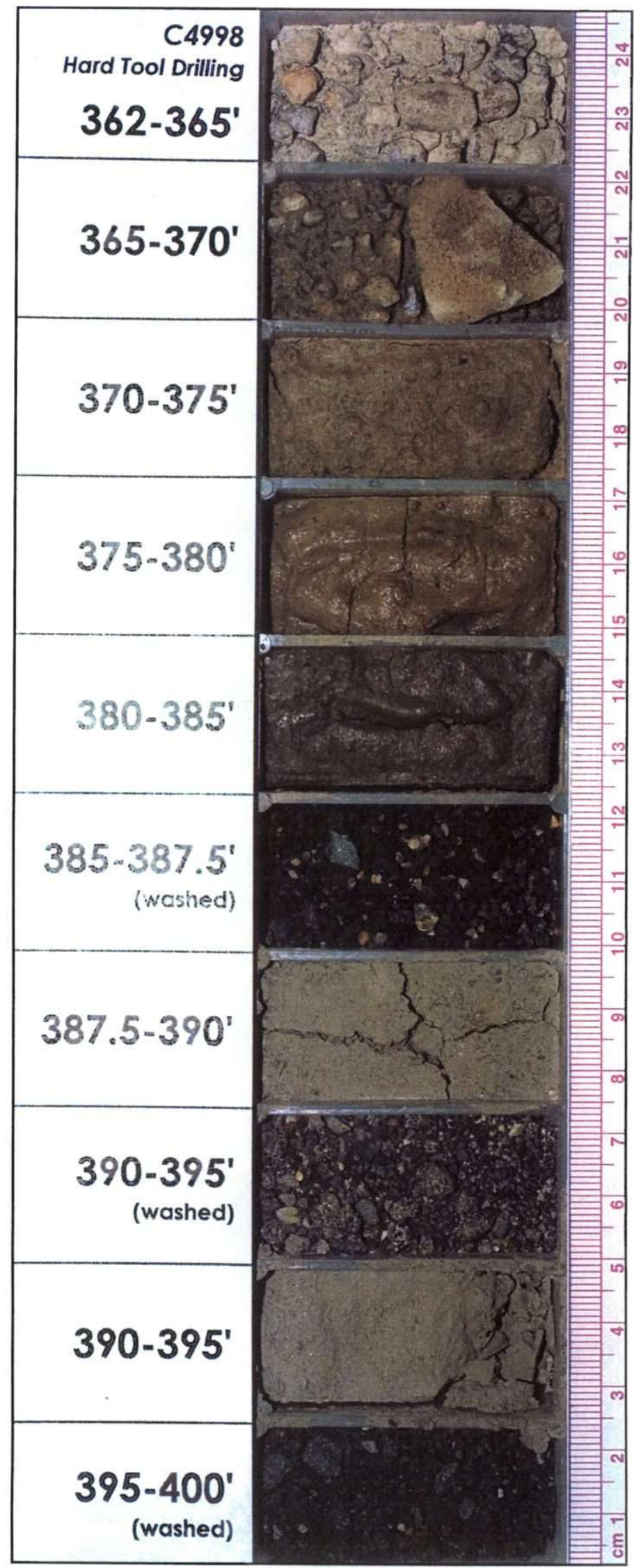

H- 8 
WMP-32119, Rev. 0

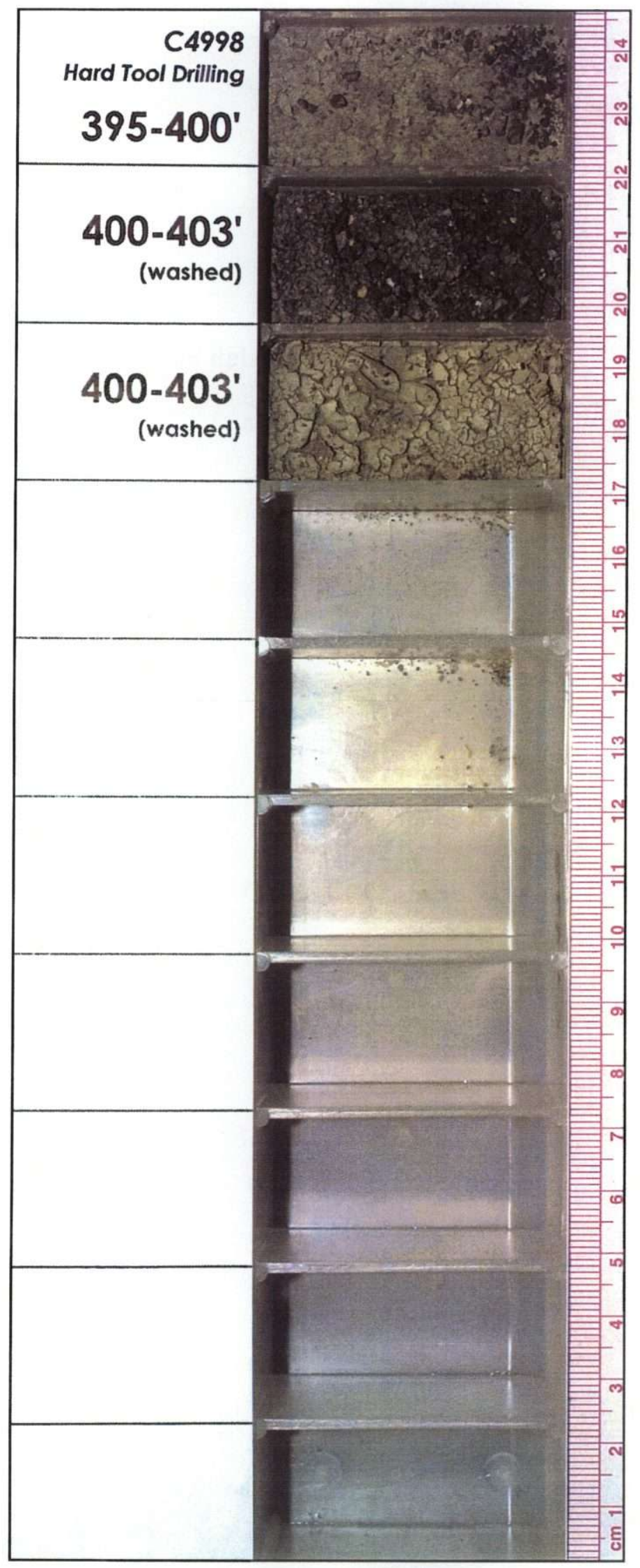

H- 9 
WMP-32119, Rev. 0

This page intentionally left blank 
WMP-32119, Rev. 0

\section{DISTRIBUTION}

\section{Onsite}

$1 \quad$ U.S. Department of Energy

Richland Operations Office

DOE Public Reading Room

$\mathrm{H} 2-53$

6 Fluor Hanford, Inc.

J. V. Borghese

E6-35

L. J. Farrell

E6-35

L. C. Swanson

E6-35

C. S. Wright

E6-35

1 Lockheed Martin Services, Inc.

Document Processing Center

H6-08

$6 \quad$ Pacific Northwest National Laboratory

Hanford Technical Library P8-55

D. B. Barnett

K6-75

T. M.Brouns

K6-69

S. P. Reidel

(3)

K6-75

6 Freestone Environmental Services, Inc.

Project Library

B2-63

S. C. Adams

B2-63

S. T. Ahlquist

B2-63

B. J. Garcia

B2-63

C. F. Rust

B2-63

J. R. Fetters

B2-63 
WMP-32119, Rev. 0

This page intentionally left blank

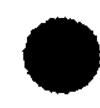

Distr - ii 